\title{
RAPPORT DU SECRÉTAIRE GÉNÉRAL AUX MINISTRES 2013
}




\section{Rapport du Secrétaire général aux ministres}

2013

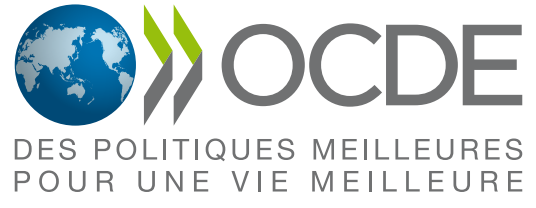


Cet ouvrage est publié sous la responsabilité du Secrétaire général de l'OCDE. Les opinions et les interprétations exprimées ne reflètent pas nécessairement les vues de l'OCDE ou des gouvernements de ses pays membres.

Ce document et toute carte qu'il peut comprendre sont sans préjudice du statut de tout territoire, de la souveraineté s'exerçant sur ce dernier, du tracé des frontières et limites internationales, et du nom de tout territoire, ville ou région.

\section{Crédits photo :}

Toutes les photos sont $\odot$ OCDE sauf

page $8 \odot$ Bundesregierung/Guido Bergmann

page 13 (c) Council of Europe

page 16 ( Laboratorio fotografico Chigi

page 19 ( ) G20/Mexique

page $20 \odot$ La Moncloa - Gobierno de España

page 21 @ G20/Russia

page $22 \odot$ Pierre Chabaud/Matignon

page 23 @ Prime Minister's Office

page 24 ( Droits réservés

page 25 @ AFP/Maxim Shemetov

Les corrigenda des publications de l'OCDE sont disponibles sur : www.oecd.org/editions/corrigenda.

\section{(C) OCDE 2013}

Vous êtes autorisés à copier, télécharger ou imprimer du contenu OCDE pour votre utilisation personnelle. Vous pouvez inclure des extraits des publications, des bases de données et produits multimédia de l'OCDE dans vos documents, présentations, blogs, sites Internet et matériel d'enseignement, sous réserve de faire mention de la source OCDE et du copyright. Les demandes pour usage public ou commercial ou de traduction devront être adressées à rights@oecd.org. Les demandes d'autorisation de photocopier une partie de ce contenu à des fins publiques ou commerciales peuvent être obtenues auprès du Copyright Clearance Center (CCC) info@copyright.com ou du Centre français d'exploitation du droit de copie (CFC) contact@cfcopies.com. 


\section{Table des matières}

\section{VUE D'ENSEMBLE}

L'OCDE en bref

Orientations stratégiques

du Secrétaire général

Activités du Secrétaire général en 2012

Réunion du Conseil

au niveau des ministres 2012

Conclusions ministérielles

Réunion du Conseil de l'OCDE

au niveau des ministres 2012

Résumé de la Présidence

Cabinet du Secrétaire général

Organigramme

\section{ACTIVITÉS PRINCIPALES}

Nouvelles approches face aux défis économiques (NAEC)

Cabinet du Secrétaire général

Bureau du Sherpa

" Penser horizontal » - Initiatives et projets de l'OCDE pour mieux relever les défis politiques interconnectés

La Stratégie pour une croissance verte

La Stratégie pour l'innovation

La Stratégie sur les compétences

L'Initiative sur la parité

La Stratégie pour le développement

L'Initiative du vivre mieux

Direction de la coopération pour le développement

Département des affaires économiques

Direction de l'éducation

et des compétences

Direction de l'emploi, du travail

et des affaires sociales
Centre pour l'entrepreneuriat,

les PME et le développement local

72

Direction de l'environnement

74

Direction des affaires financières

et des entreprises

Direction de la gouvernance publique

et du développement territorial

\section{AGENCES DE L'OCDE}

\section{ET AUTRES ENTITÉS SPÉCIALES}

Centre de développement

Agence pour l'énergie nucléaire

Agence internationale de l'énergie

Forum pour le partenariat avec l'Afrique $\quad 100$

Groupe d'action financière 100

Forum international des transports

Club du Sahel et de l'Afrique de l'Ouest

Comité consultatif économique

et industriel

Commission syndicale consultative 


\section{L'OCDE en bref}

\section{www.oecd.org/apropos}

webmaster@oecd.org

L'OCDE est un forum où les gouvernements travaillent ensemble à rechercher des solutions à des problèmes communs, partager leurs expériences et identifier les meilleures pratiques afin de promouvoir des politiques meilleures pour une vie meilleure.

Depuis plus de 50 ans, l'OCDE a contribué à élaborer des normes mondiales, des conventions internationales, des accords et des recommandations dans des domaines tels que, notamment, la gouvernance et la lutte contre la corruption, la responsabilité des entreprises, le développement, l'investissement international, la fiscalité et l'environnement. L'OCDE s'appuie sur la coopération, le dialogue, le consensus et les examens entre pairs pour faire avancer sa vision d'une économie et d'une société mondiales plus fortes, plus propres et plus justes.

L'OCDE aide les responsables publics à identifier les enjeux et à y faire face par des mesures appropriées. Elle dispense également des conseils sur presque tous les aspects de l'élaboration et de la mise en œuvre des politiques, et représente, au niveau mondial, l'une des plus importantes et plus fiables sources de données statistiques comparables sur les questions économiques et commerciales, l'emploi, l'éducation, la santé, les questions sociales, les migrations, l'environnement et bien d'autres domaines couverts par ses comités d'experts nationaux et un Secrétariat de grande qualité.

L'OCDE compte 34 pays membres et a engagé des discussions en vue de l'adhésion avec la Fédération de Russie. L'Afrique du Sud, le Brésil, la République populaire de Chine, l'Inde et l'Indonésie sont des partenaires clés de l'OCDE. L'OCDE collabore également avec plus d'une centaine d'autres économies, dont beaucoup participent aux travaux de ses comités et adhèrent à ses instruments.

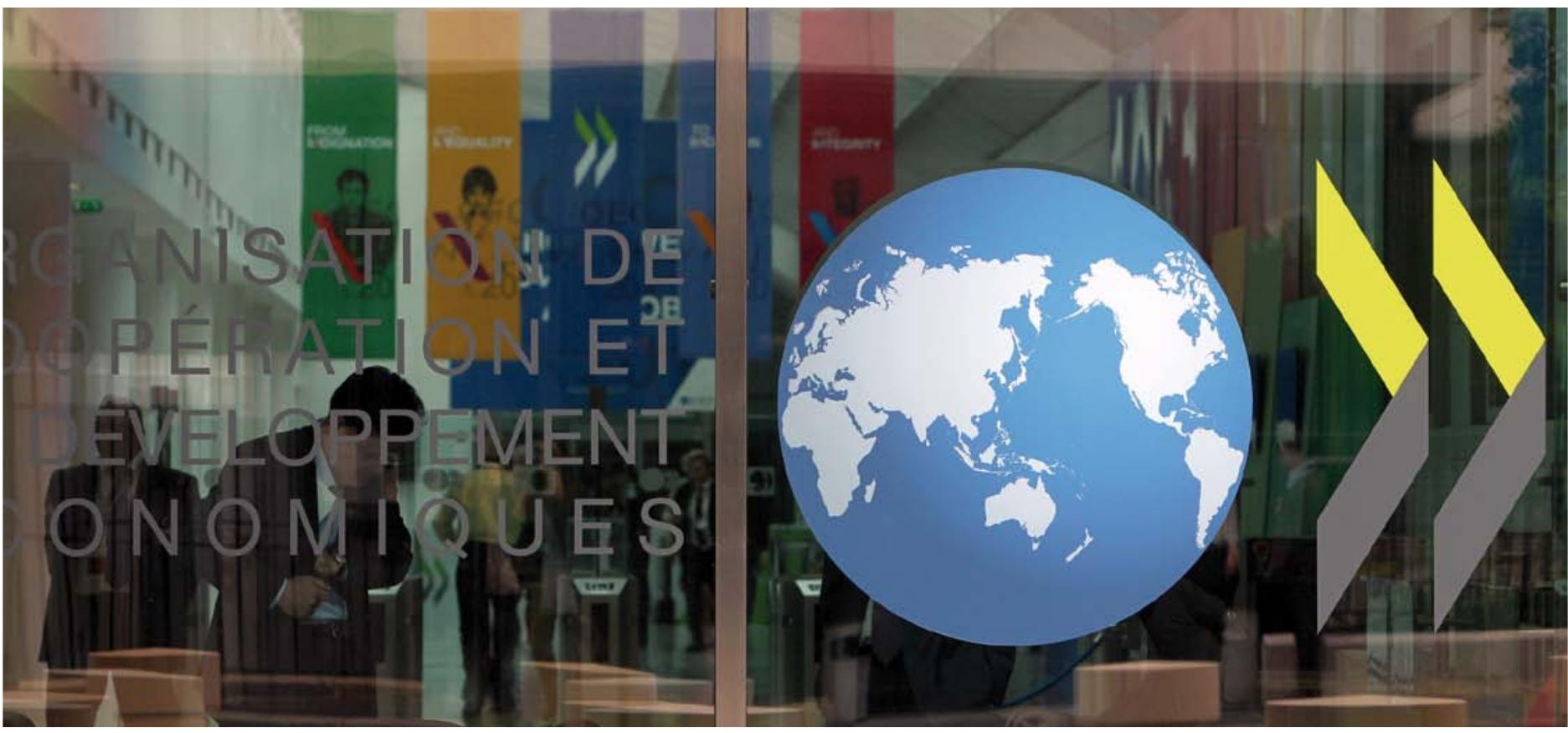


Pays membres de l'OCDE : Ambassadeurs auprès de l'OCDE* et dates d'adhésion

\section{www.oecd.org/paysmembres}

\begin{tabular}{|c|c|c|}
\hline Allemagne & Son Excellence M. Hans-Juergen Heimsoeth & 1961 \\
\hline Australie & Son Excellence M. Chris Barrett & 1971 \\
\hline Autriche & Son Excellence M. Wolfgang Petritsch & 1961 \\
\hline Belgique & Son Excellence M. Yves Haesendonck & 1961 \\
\hline Canada & Son Excellence Mme Judith LaRocque & 1961 \\
\hline Chili & Son Excellence M. Raúl Sáez & 2010 \\
\hline Corée & Son Excellence M. Kyung Wook Hur & 1996 \\
\hline Danemark & Son Excellence M. Poul Erik Dam Kristensen & 1961 \\
\hline Espagne & Son Excellence M. Ricardo Díez-Hochleitner & 1961 \\
\hline Estonie & Son Excellence M. Marten Kokk & 2010 \\
\hline États-Unis & Mme Jeri Guthrie-Corn (Chargée d'affaires, a.i.) & 1961 \\
\hline Finlande & Son Excellence M. Antti Kuosmanen & 1969 \\
\hline France & Son Excellence Mme Pascale Andréani & 1961 \\
\hline Grèce & Son Excellence Mme Konstantina Birmpili & 1961 \\
\hline Hongrie & Son Excellence M. István Mikola & 1996 \\
\hline Irlande & Son Excellence M. Michael Forbes & 1961 \\
\hline Islande & Son Excellence Mme Berglind Ásgeirsdóttir & 1961 \\
\hline Israël & Son Excellence M. Nimrod Barkan & 2010 \\
\hline Italie & Son Excellence M. Carlo Maria Oliva & 1962 \\
\hline Japon & Son Excellence M. Motohide Yoshikawa & 1964 \\
\hline Luxembourg & Son Excellence M. Paul Dühr & 1961 \\
\hline Mexique & Son Excellence M. Dionisio Pérez-Jácome & 1994 \\
\hline Norvège & Son Excellence M. Tore Eriksen & 1961 \\
\hline Nouvelle-Zélande & Son Excellence Mme Rosemary Banks & 1973 \\
\hline Pays-Bas & Son Excellence M. Edmond $\mathrm{H}$. Wellenstein & 1961 \\
\hline Pologne & Son Excellence M. Pawel Wojciechowski & 1996 \\
\hline Portugal & Son Excellence M. Paulo Vizeu Pinheiro & 1961 \\
\hline République slovaque & Son Excellence Mme Ingrid Brocková & 2000 \\
\hline République tchèque & Son Excellence M. Pavel Rozsypal & 1995 \\
\hline Royaume-Uni & Son Excellence M. Nicholas Bridge & 1961 \\
\hline Slovénie & Son Excellence M. Andrej Rant & 2010 \\
\hline Suède & Son Excellence M. Anders Ahnlid & 1961 \\
\hline Suisse & Son Excellence M. Stefan Flückiger & 1961 \\
\hline Turquie & Son Excellence M. Kadri Ecvet Tezcan & 1961 \\
\hline Union européenne & Son Excellence Mme Maria Francesca Spatolisano & 1961 \\
\hline
\end{tabular}

\section{Candidat à l'adhésion}

www.oecd.org/adhesion

Fédération de Russie

\section{Partenaires clés}

\section{www.oecd.org/fr/relationsmondiales/partenairescles}

Afrique du Sud

Brésil

Chine, République populaire de

Inde

Indonésie 


\section{Orientations stratégiques du Secrétaire général}

\section{Résumé}

Dans le droit fil de la « Vision d'avenir » exprimée à l'occasion du $50^{\mathrm{e}}$ anniversaire de l'OCDE en 2011, mes Orientations stratégiques pour 2013 s'articulent autour de trois maîtres-mots - IMPACT, INCLUSIVITÉ ET MISE EN ÆUVRE de façon à nous donner les moyens de continuer d'aider nos membres et nos pays partenaires à concevoir, promouvoir et mettre en œuvre des " politiques meilleures pour une vie meilleure ".

Alors que nous ne sommes pas encore sortis d'affaire et que nombre de nos concitoyens souffrent encore des conséquences de la crise, il est essentiel que les pays ne ralentissent pas leur effort de réforme, afin d'étayer la reprise, de favoriser la croissance à long terme, de renforcer leur résistance face à de nouvelles crises, et de renforcer la confiance, les espérances et les perspectives de leur population. Il est impératif de renouer avec la croissance, de faire reculer le chômage, de résorber les inégalités et de rétablir la confiance pour ouvrir des perspectives d'avenir plus favorables. Dans le même temps, nous devons prendre acte des nouvelles tendances qui se font jour et y faire face : la poursuite de la mondialisation, qui s'accompagne d'un basculement de la richesse, le vieillissement de la population, les courants migratoires, la raréfaction des ressources naturelles et le changement climatique, et un biais, induit par le progrès technologique, en faveur de la connaissance et des compétences.

Pour avoir de l'IMPACT, l'action de l'OCDE doit s'appuyer sur son atout le plus précieux, le caractère multidimensionnel de ses travaux, afin de produire des analyses, des conseils et des normes internationales de nature à promouvoir une croissance économique plus forte, plus saine et plus juste. Nous devons donc envisager la notion de croissance économique dans une perspective qui place l'égalité, la durabilité et le bien-être aux tous premiers rangs, et qui favorise l'ouverture des marchés, tout en aidant les pays à mieux prendre en compte et exploiter les réalités de l'économie mondiale d'aujourd'hui.

L'initiative relative aux Nouvelles approches face aux défis économiques (NAEC) est l'échafaudage qui nous permettra de mener à bien notre chantier de rénovation continue grâce à une amélioration de nos grilles d'analyse et de nos conseils sur les politiques à suivre. Depuis le lancement de l'initiative NAEC à la RCM de 2012, nous avons considérablement progressé. Nous devons maintenant inscrire l'initiative dans un cadre cohérent propre à en fédérer les différentes composantes autour d'un axe logique d'où se dégagent des solutions permettant de mieux appréhender des objectifs concurrents et de mettre à profit les synergies, de suivre et d'adapter les dernières évolutions des politiques publiques et des développements théoriques susceptibles d'avoir une utilité pour nos travaux, et d'intégrer les réalités inhérentes aux économies émergentes et en développement dans nos données, nos analyses et nos recommandations.

L'IMPACT que nous recherchons doit s'appuyer sur nos atouts incontestables et venir consolider les initiatives existantes. Nos projets " transversaux » - la nouvelle norme au sein de l'Organisation - jouent de plus en plus comme une force d'attraction qui nous permettra d'assembler et de mieux organiser les travaux de l'ensemble de l'Organisation. Nous avons 


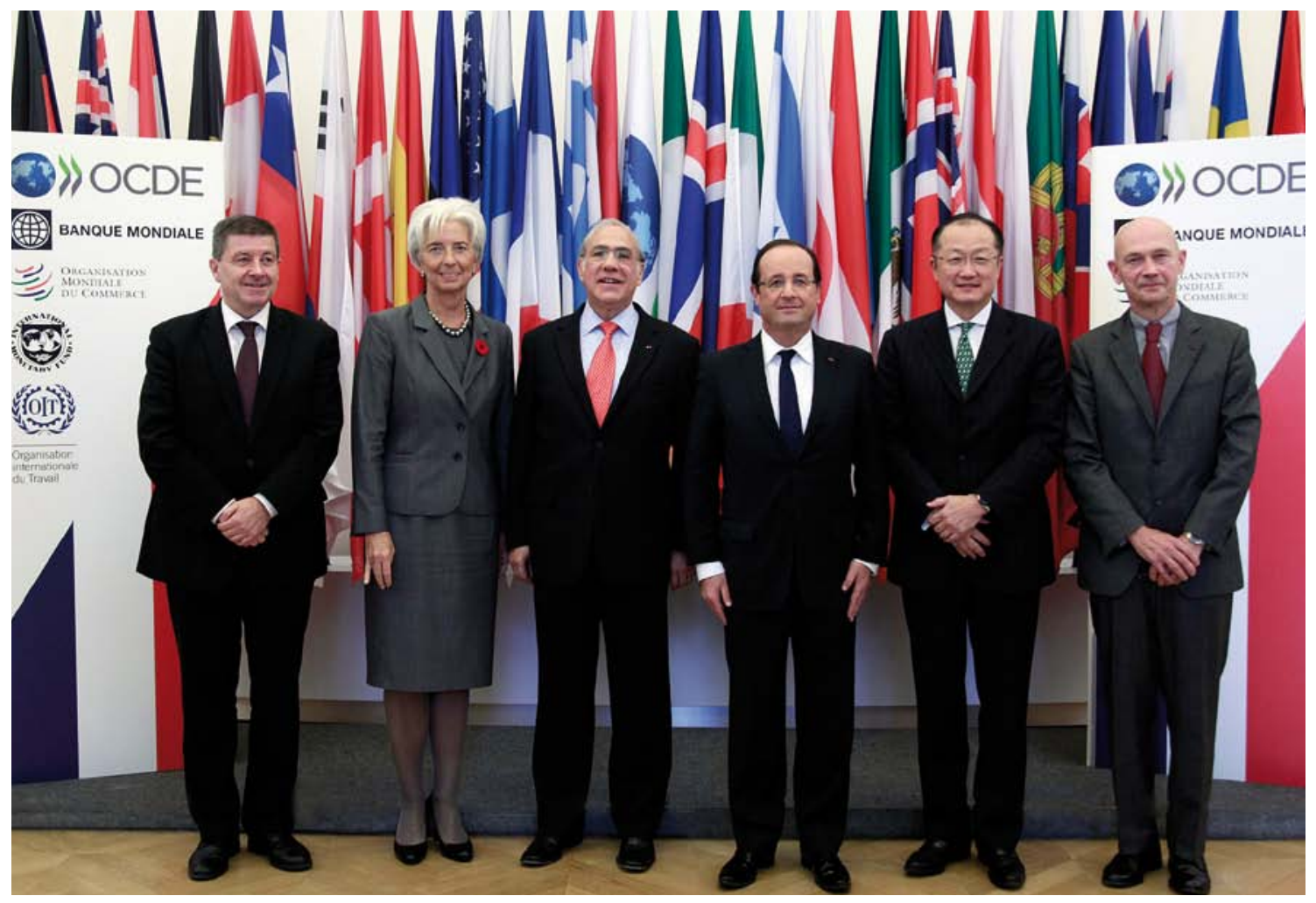

(gauche/droite) Guy Ryder, Directeur general de l'OIT ; Christine Lagarde, Directrice générale du FMI ; Angel Gurría, Secrétaire général de l'OCDE ; François Hollande, Président de la République française ; Jim Yong Kim, Président de la Banque mondiale et Pascal Lamy, Directeur général de l'OMC, à l'OCDE en octobre 2012

progressé avec la Stratégie pour le développement pour ce qui concerne la cohérence des politiques au service du développement, le renforcement de l'engagement et le partage des connaissances avec les pays en développement, mais il y a encore beaucoup de chemin à parcourir pour véritablement intégrer le développement dans nos travaux. Nous nous employons actuellement à intégrer la Stratégie pour une croissance verte dans nos axes de travail centraux, mais il faut poursuivre les travaux afin de mettre au point des indicateurs et autres outils de mesure, de renforcer nos analyses sectorielles et d'intégrer la problématique de la croissance verte dans les politiques publiques à l'échelon aussi bien infranational que multilatéral.
Notre Stratégie sur les compétences suit une approche par pays, notre initiative pour la parité aboutira à des réalisations concrètes sous la forme d'une Recommandation sur l'égalité homme-femme à la RCM de cette année, et nous nous apprêtons également à reconsidérer et actualiser notre Stratégie pour l'innovation.

La " croissance inclusive " est une pièce essentielle du puzzle. Nous devons nous placer dans une perspective véritablement multidimensionnelle pour instaurer une croissance qui place l'égalité, la durabilité et le bien-être aux tous premiers rangs. C'est pourquoi nos travaux consacrés à la croissance inclusive viseront en priorité à déterminer comment définir au mieux le concept, le mesurer et le comparer, et apporter des éclairages sur les options qui s'offrent aux pouvoirs publics et les arbitrages qu'ils devront opérer afin de promouvoir la croissance tout en préservant son caractère INCLUSIF.

Les analyses structurelles et les recommandations visant à promouvoir la croissance, l'emploi et le bien-être continueront d'être le ciment de l'action de l'Organisation. Dans ce contexte, je suis déterminé à affiner encore nos travaux sur les divers aspects que recouvre la notion de compétitivité, allant de la concurrence à la productivité ; de l'éducation aux compétences ; des réformes du marché du travail à celles des marchés de produits ; des marchés nationaux aux marchés 
internationaux ; ou encore de l'innovation à une utilisation plus efficiente des ressources naturelles. Dans la conjoncture économique actuelle, nos efforts porteront en premier lieu sur des domaines tels que les politiques actives en faveur des sans-emploi, l'amélioration de la qualité des emplois, un Plan d'action pour l'emploi des jeunes, les migrations, et la résorption des inégalités, y compris entre les hommes et les femmes.

Nous devons également continuer d'élargir le champ des conseils que nous prodiguons aux pays pour les aider à réagir face à l'interdépendance et à la complexité croissantes de l'économie mondiale. Nous devrions aider les gouvernements à dynamiser ou stimuler leurs économies en parant aux risques et en saisissant les opportunités qui découlent de cette complexité, et en misant tout particulièrement sur de nouvelles sources de croissance. Au cours des années qui viennent, nous mettrons encore plus l'accent sur les nouvelles priorités, riches de promesses, que sont les actifs intellectuels, les chaînes de valeur mondiales et les échanges exprimés en valeur ajoutée, la croissance verte, et l'Initiative du vivre mieux, laquelle intégrera les sports et la culture. Les politiques fiscales devront aussi être adaptées à une économie de plus en plus mondialisée, afin d'assurer des recettes budgétaires adéquates et de préserver la santé des finances publiques. Nos travaux sur l'érosion des bases d'imposition et le transfert des bénéfices (BEPS) constitueront un jalon important dans la concrétisation de cette ambition.

Pour améliorer la MISE EN ÆUVRE, et par là même produire davantage d'IMPACT, il est impératif de pouvoir compter sur des institutions efficaces. Sans des institutions fortes, avisées et fiables, nos efforts pour mettre en œuvre des politiques meilleures pour une vie meilleure seront compromis. L'OCDE devrait accompagner les efforts déployés pour mettre en place des institutions publiques plus efficaces, plus transparentes et plus ouvertes. Nous nous attacherons en particulier à instaurer une gouvernance plus avisée et plus efficace en mettant en place une réglementation de meilleure qualité, en optimisant davantage les ressources et en misant sur les nouvelles méthodes et les nouvelles technologies pour faciliter et accompagner la planification et la prise de décisions. Renforcer la transparence et la responsabilité financières permettrait aux gouvernements de montrer clairement qu'ils sont déterminés à regagner la confiance des citoyens. C'est pourquoi nous avons l'intention d'étudier des aspects déterminants tels que le financement des campagnes électorales et son impact sur la fixation des priorités politiques et sur les processus de décision. Une amélioration du gouvernement d'entreprise en général, et dans le secteur financier en particulier, reste également fondamentale si l'on veut atteindre ces objectifs.

Nous renforcerons notre IMPACT en développant une approche par pays. En 2012, nous avons

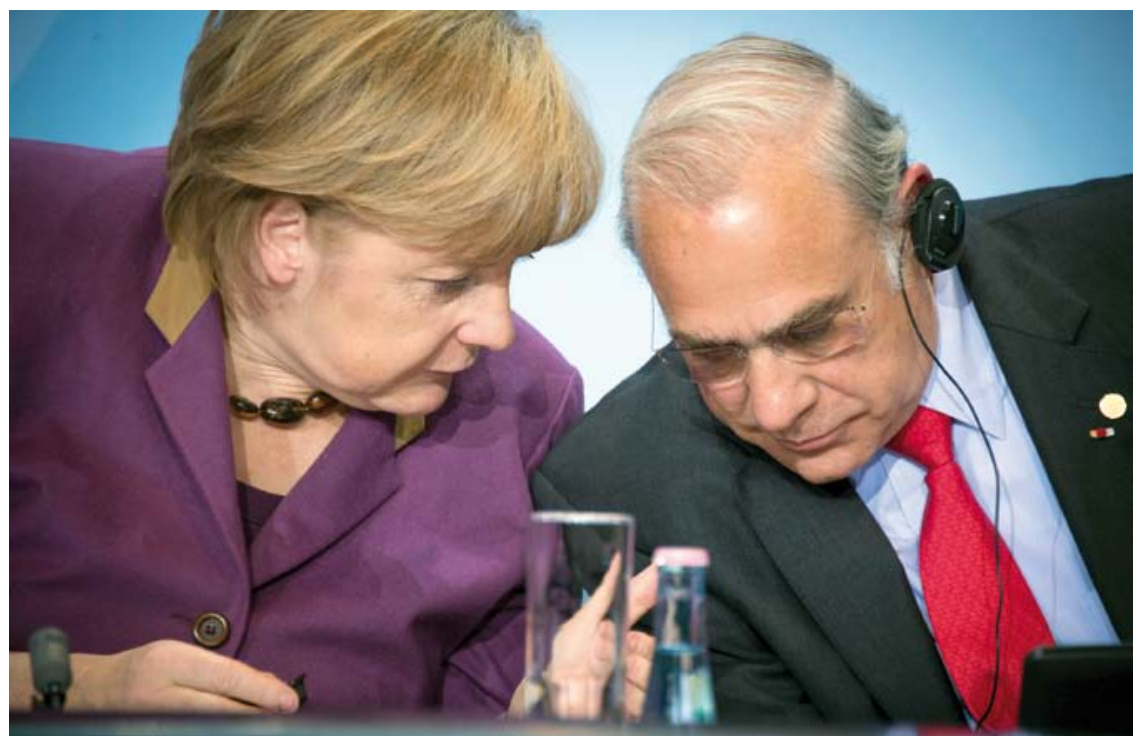


considérablement accru notre impact sur les programmes d'action nationaux et avons été le partenaire de nos gouvernements au plus haut niveau, œuvrant à leurs côtés pour créer des solutions sur mesure face aux difficultés qu'ils rencontrent. Pour l'avenir, nous avons l'objectif de renforcer ce rôle. En particulier, il nous faut axer encore davantage nos travaux sur le volet MISE EN EUUVE, en améliorant le concours que nous apportons aux responsables de l'action publique par des programmes de renforcement des capacités, des ateliers et des formations. Compte tenu de l'intérêt grandissant pour l'OCDE dont témoignent les personnalités de haut rang en se rendant en visite à l'Organisation, nous mettrons en outre à profit notre expérience pour organiser de tels événements dans le cadre d'un " Programme spécial consacré aux personnalités de haut rang et à leur vision du monde ». Publiée en différentes langues, notre nouvelle série de publications intitulée OCDE $360^{\circ}$, offrira un panorama des principaux domaines sur lesquels portent nos travaux dans chaque pays membre. Nous poursuivrons ces travaux en tenant dûment compte de la spécificité des besoins, des situations et du cadre institutionnel des pays.

Il faut certes développer une approche par pays, tout en continuant à avoir une perspective mondiale et prolonger notre Vision d'avenir du 50 anniversaire de l'Organisation pour devenir un " réseau mondial pour l'action publique plus efficace et plus INTÉGRATEUR ». S'agissant des partenaires clés, il convient de renforcer les liens existants et de continuer à travailler sur des outils spécifiques ou attachés à des projets, tels que l'Accord cadre avec l'Indonésie. Nous poursuivrons nos

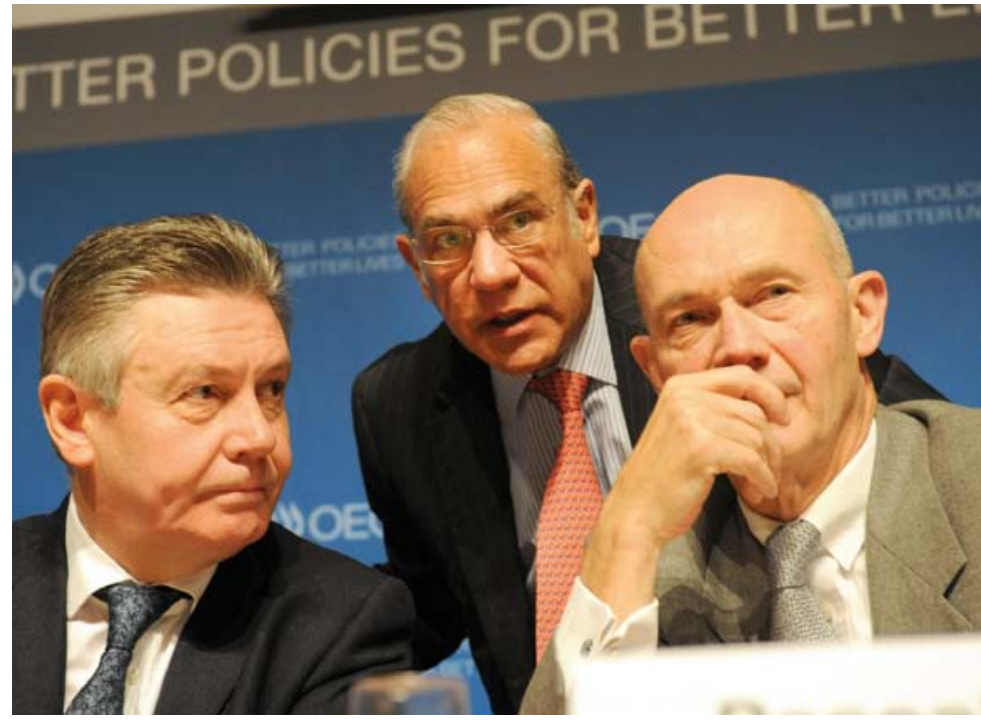

Karel de Gucht, Commissaire européen au Commerce, Angel Gurría, Secrétaire général de l'OCDE, et Pascal Lamy, Directeur général de l'OMC, à la conférence de presse OCDE-OMC sur l'Initiative sur les Échanges en valeur ajoutée (ÉVA) en janvier 2013

travaux avec la Fédération de Russie pour mener à son terme le processus d'adhésion engagé, et nous espérons pouvoir compter sur une décision quant à l'ouverture de nouvelles discussions en vue de l'adhésion avec d'autres pays intéressés dans un avenir proche. Dans une perspective régionale, nous continuerons d'appuyer les réformes dans les pays de la région MENA, d'Afrique et d'Amérique latine, et déploierons des efforts particuliers pour renforcer et structurer nos relations avec l'Asie du Sud-Est. Nous continuerons de fournir, en temps opportun et en toute efficacité, des analyses à destination de toutes les enceintes internationales concernées, notamment le G20, le $\mathrm{G} 8$, le Partenariat mondial pour une coopération efficace au service du développement, les cadres dans lesquels s'inscriront les objectifs post-2015 et les objectifs de développement durable, ou encore les négociations sur la CCNUCC. Enfin, nous nous appliquerons à renforcer notre rôle d'instance internationale d'établissement de normes, en nous appuyant sur les normes existantes, en repérant les lacunes et les besoins qui se font jour à l'échelle mondiale dans ce domaine, et nous examinerons où et comment l'OCDE pourrait apporter une contribution utile.

Dernier point, mais pas le moindre, une "Stratégie pour l'efficience " et une "Efficience stratégique " sont essentielles si l'on veut produire un IMPACT pour tous nos membres. Nous continuerons en particulier d'assurer une utilisation optimale des ressources de nos membres tout en agissant avec efficience dans un contexte budgétaire difficile. Nos services internes continueront d'aider l'Organisation à atteindre ses objectifs stratégiques de diverses façons, notamment par le recrutement, la gestion des talents et la gestion des connaissances, et nous veillerons à ce que l'ensemble des données de l'OCDE soient ouvertes, accessibles et gratuites d'ici la mi2015. 


\section{Orientations stratégiques du Secrétaire général}

\section{Section principale}

Cette note présente mes analyses sur les priorités stratégiques qui seront celles de l'Organisation de coopération et de développement économiques (OCDE) pour l'année à venir et au-delà. Ces priorités sont dictées par le contexte général actuel, les principaux défis auxquels doivent faire face les pays membres et les pays partenaires, et les réponses qu'il convient d'y apporter. Elles s'inspirent aussi des discussions que j'ai eues avec des dirigeants, ministres et chefs de délégation, ainsi qu'avec d'autres parties prenantes des secteurs public et privé, ou de la société civile.

\section{Perspectives mondiales et enjeux}

Des choix fondés sur des données concrètes, une impulsion politique et une action résolue, tels sont les éléments essentiels qui doivent présider à l'élaboration d'un scénario post-crise qui donne des résultats. L'atonie de la croissance, l'ampleur du chômage, la fragilité des finances publiques, le niveau insoutenable de l'endettement public et privé, ou encore les risques qui entourent le secteur financier demeurent autant de menaces pour de nombreuses économies avancées. Tant que l'activité ne se redresse pas fermement, nous ne pouvons prétendre être définitivement sortis de la crise. Il est impératif dans les économies aussi bien avancées qu'émergentes de promouvoir l'emploi et de remédier aux inégalités qui continuent de se creuser. La dimension sociale de la crise est patente, et la confiance dans l'aptitude des pouvoirs publics à renverser la situation est à reconstruire. Renouer avec la croissance, réduire le chômage et le sous-emploi, en particulier chez les jeunes et les chômeurs de longue durée, recréer un climat de confiance et résorber les inégalités, telles seront les clés qui nous ouvriront les portes d'un avenir meilleur.

Nous devons faire face à des tendances mondiales qui continueront de façonner non seulement la réalité internationale mais aussi les stratégies nationales, tout en servant au mieux les intérêts de tous nos concitoyens : au nombre d'entre elles figurent la mondialisation, qui s'accompagne d'un basculement de la richesse, et qui s'enracine sans cesse davantage ; le vieillissement de la population, les courants migratoires et autres évolutions démographiques ; la raréfaction des ressources naturelles et l'aggravation des problèmes environnementaux ; l'infléchissement de l'économie mondiale, induit par le progrès technologique, où les connaissances et les compétences jouent un rôle primordial. Il sera absolument essentiel d'intensifier les efforts déployés à l'échelon national et international pour créer les conditions d'une amélioration de la croissance et de l'emploi, retrouver la stabilité budgétaire et corriger les déséquilibres, tout en nous efforçant, entre autres, de parer au changement climatique, de renforcer le système commercial multilatéral, de lutter contre le protectionnisme, d'améliorer l'inclusivité et la cohésion sociales et de favoriser le développement. 
Mais la nouvelle donne économique et les mégatendances qui se font jour exigent de notre part une capacité à voir plus loin. Nos systèmes politiques, économiques, sociaux et statistiques doivent mieux prendre en compte un monde de plus en plus complexe et interconnecté, et rester en phase avec les nouvelles évolutions mondiales. Pour mieux appréhender ces nouvelles réalités, nous devons revoir nos grilles d'analyse, remettre en question les " vérités établies " et les analyses conventionnelles, tout en réexaminant et en peaufinant les conseils que nous prodiguons. C'est bien l'ambition à laquelle nous souhaitons donner corps avec l'initiative relative à de Nouvelles approches face aux défis économiques (NAEC) lancée à l'occasion de la réunion du Conseil au niveau des ministres (RCM) de 2012.

Ces Orientations stratégiques ont donc pour objet de mettre à profit la nature multidimensionnelle de notre Organisation afin de refléter l'interdépendance de nos économies, de relier les divers aspects stratégiques des travaux de l'OCDE et de promouvoir une définition globale de la croissance et des enjeux économiques qui place en son centre le bien-être, l'équité et le développement durable. Notre aptitude à renforcer encore ces imbrications horizontales entre différents domaines de l'action publique constitue l'un de nos principaux atouts et distingue l'OCDE des autres organisations internationales. C'est pourquoi l'OCDE devrait tirer le meilleur parti des initiatives et des projets transversaux consacrés par exemple à la croissance verte, à l'innovation, aux compétences, au développement et aux nouvelles approches face aux défis économiques, lesquels nous permettent de mieux fédérer les travaux de l'Organisation.
Les Nouvelles approches face aux défis économiques : un programme d'action de l'OCDE pour une croissance inclusive

L'initiative NAEC contribuera à renforcer le cadre stratégique dans lequel s'inscriront les travaux de l'OCDE pour l'avenir. Cet exercice de réflexion à l'échelle de l'ensemble de l'Organisation sera le moteur continu d'une amélioration de nos grilles d'analyse et des conseils que nous prodiguons. Dans le aussi pour améliorer le bien-être des populations, favoriser une répartition équitable des fruits de la croissance et assurer que les ressources naturelles sont utilisées de manière durable à long terme.

Il ne s'agit pas d'un projet qui sera classé une fois achevé et à l'issue duquel nous reprendrons le cours de nos activités. C'est un " état d'esprit » que de remettre continuellement en question nos propres hypothèses et de nous tenir prêts à tirer des enseignements des

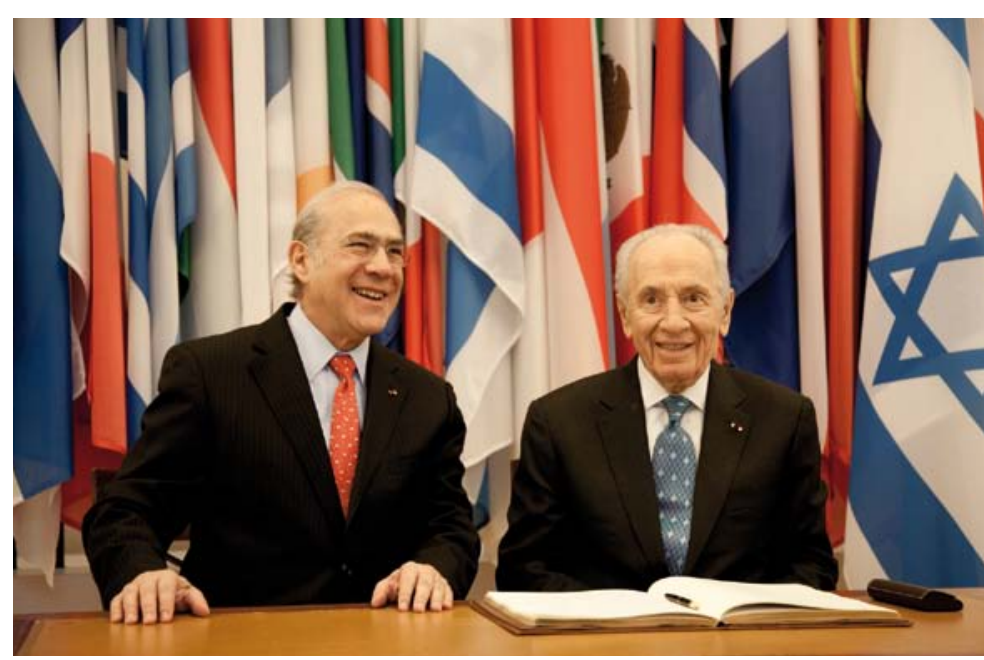

Le Secrétaire général de l'OCDE Angel Gurría avec Shimon Peres, Président d'Israël, pendant la visite officielle du Président à l'OCDE en mars 2013

cadre de cette initiative, nous nous appliquerons à revoir nos hypothèses fondamentales quant au fonctionnement de l'économie et à améliorer nos outils et modèles pour en corriger les lacunes et en étendre les capacités. Nous nous attacherons en particulier à mieux tenir compte des interactions et des retours d'expérience dans nos analyses. Nous entendons affiner encore nos conseils sur l'action à mener non seulement pour renouer avec une croissance plus forte, mais avancées scientifiques, sociales et économiques, ainsi que des idées nouvelles venant de tous les secteurs de l'Organisation, des pays membres et des pays partenaires. Cet état d'esprit devrait continuer d'imprimer sa marque à l'ensemble des travaux de l'OCDE afin que l'Organisation produise des analyses et des recommandations de qualité. Depuis le lancement de l'initiative NAEC à l'occasion de la RCM de 2012, nous avons accompli des progrès substantiels. Nous 
avons mis en place une structure d'organisation permettant de tirer les leçons de la crise, d'engager une réflexion prospective pour faire face aux enjeux de demain, d'analyser les principaux arbitrages et complémentarités entre les politiques publiques, et d'examiner certaines des plus importantes problématiques, qui exigent notre attention, relatives aux institutions et à la gouvernance.

Le principal enjeu au cours de l'année à venir consistera à inscrire l'initiative NAEC dans un cadre cohérent propre à en fédérer les différentes composantes autour d'un axe logique. Plus particulièrement, nous devons en priorité rechercher les moyens de mieux appréhender des objectifs qui peuvent entrer en concurrence - notamment entre la croissance et les inégalités, la viabilité des finances publiques et les inégalités, la croissance et l'environnement, l'environnement et les inégalités, ou encore la croissance, l'(in)stabilité économique et le risque - et de mieux exploiter les synergies. Deuxièmement, il nous faut prendre en compte dans nos travaux les nouvelles approches de l'économie, telles que l'économie comportementale, les théories de la complexité, les travaux que nous avons engagés pour mesurer le bien-être au-delà du seul PIB, et les enseignements d'autres disciplines. Troisièmement, nous devons systématiquement replacer nos analyses et nos conseils dans une perspective institutionnelle, de façon à être mieux à même de déterminer les capacités ou les contraintes qui favoriseront ou au contraire freineront la mise en œuvre et la production de ces conseils et analyses. Enfin, il nous faut intégrer la réalité des économies émergentes ou en développement dans nos données, nos analyses et nos recommandations. Ce sont tous ces éléments qui aboutiront à l'élaboration d'un " programme d'action stratégique propre à

favoriser la croissance inclusive ", tel qu'envisagé par les ministres en 2012.

Nos premiers travaux relatifs à l'initiative NAEC ont également contribué à mieux cerner les domaines dans lesquels les pays membres et les pays partenaires ont le plus besoin du concours de l'OCDE. C'est pourquoi mes Orientations stratégiques pour l'année à venir auront plus particulièrement pour objet d'obtenir des résultats dans les trois grands domaines suivants:

- Inclusivité et croissance ;

- Interdépendance au service de la croissance ;
- Institutions et gouvernance au service de la croissance.

\section{Inclusivité et croissance}

\section{Croissance et emploi}

L'OCDE continue d'aider les pays à élaborer et à mettre en œuvre un programme de réformes en faveur de la croissance et de l'emploi. Nous nous sommes en particulier appuyés sur nos compétences structurelles, en mettant au point des outils visant à éliminer les blocages et les obstacles à la croissance. Nous avons mis à la disposition de nos pays membres les abondantes connaissances de l'OCDE à certains moments stratégiques de leur vie politique. Nous avons soutenu les gouvernements qui devaient prendre de difficiles

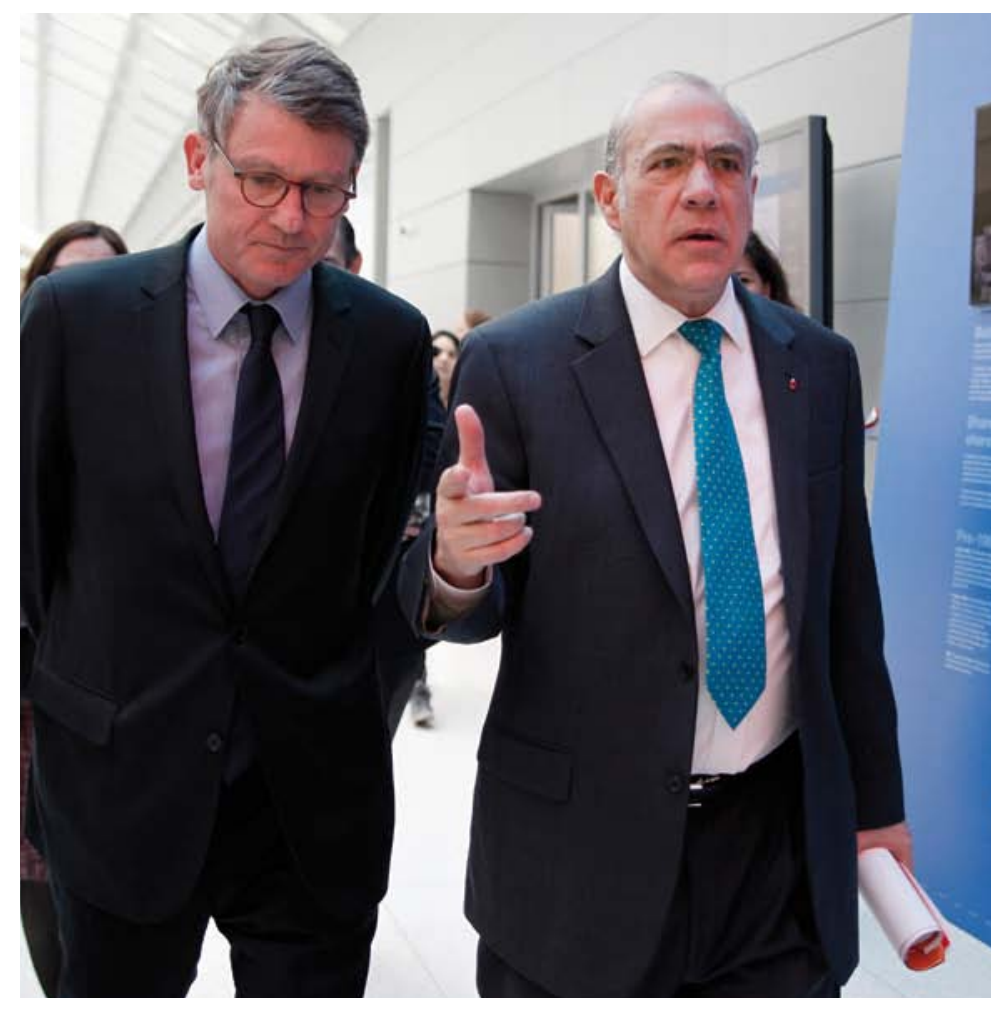

Vincent Peillon, ministre français de l'Éducation, et Angel Gurría, Secrétaire général de l'OCDE, au Colloque international sur la professionnalisation de la formation des enseignants 
décisions au service d'objectifs à long terme. Nous avons redoublé d'efforts pour soutenir des chefs d'État, des ministres et leurs gouvernements afin d'améliorer les performances de leurs économies et de mettre en œuvre les priorités de l'action publique et d'atteindre les objectifs visés par les réformes, notamment grâce à notre série Politiques meilleures, nos rapports sur la compétitivité et notre série Getting it Right.

À l'avenir, la production d'analyses structurelles et de recommandations, conjuguée à nos travaux sur les nouvelles sources de croissance et d'emploi, continuera d'être l'axe principal de notre stratégie. C'est le fil directeur de l'ensemble de nos travaux et de ces Orientations stratégiques, que complètent des propositions visant à centrer nos efforts sur un certain nombre de domaines structurels. Forte de sa crédibilité, l'OCDE est l'organisation internationale qui peut à la fois faciliter la planification des réformes et faire office de catalyseur afin de favoriser leur mise en œuvre dans les pays membres. Cette démarche englobe nos travaux traitant des divers aspects de la compétitivité - allant de la concurrence à la productivité, de l'éducation aux compétences, des réformes du marché du travail à celles des marchés de produits, des marchés nationaux aux marchés internationaux ou encore de l'innovation à l'utilisation plus efficiente des ressources naturelles. Ce sont autant d'aspects qui seront au cœur de notre stratégie pour les années à venir. Ces efforts seront étayés par les travaux que nous poursuivons sur les nouvelles sources de croissance, qui seront créatrices d'emplois et devront être écologiques, innovantes, fondées sur les connaissances et les compétences ainsi que sur l'égalité homme-femme.

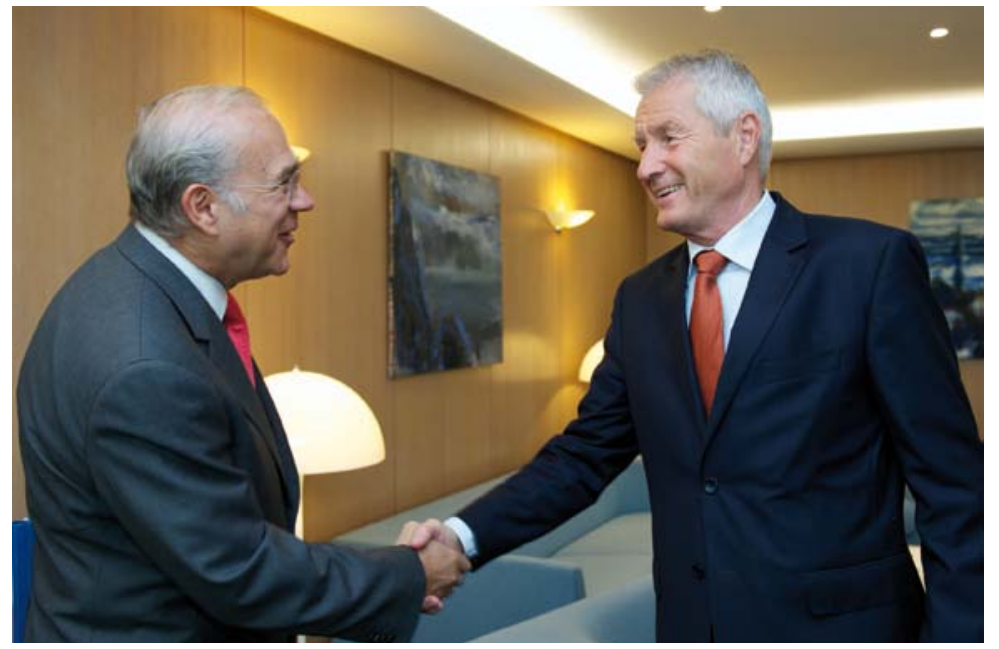

Angel Gurría, Secrétaire général de l'OCDE, est accueilli par Thorbjørn Jagland, Secrétaire général du Conseil de l'Europe, à l'occasion de l'Assemblée parlementaire en octobre 2012

Grâce à nos analyses, nous aiderons en outre les pays à mettre au point des stratégies d'assainissement des finances publiques adaptées à leur spécificité et propices à la croissance qui en atténueront les retombées préjudiciables sur la répartition du revenu et qui favoriseront la prospérité à long terme, tout en nous attaquant aux problèmes que posent sur le long terme la question des retraites, des soins de santé et de l'environnement.

\section{Croissance inclusive}

Toutefois, si la croissance économique est un moyen important pour parvenir à cette fin, elle n'est pas une fin en soi. La croissance doit devenir plus inclusive, de sorte que les disparités de résultats et d'opportunités entre les différentes catégories sociales soient moins prononcées et que les fruits de la croissance soient plus largement partagés. En œuvrant dans le cadre de l'initiative NAEC et en nous appuyant sur nos travaux phares consacrés à la croissance, aux inégalités et au bien-être, nous entendons proposer un nouvel idéal de croissance inclusive qui conjugue vigueur de la croissance économique et amélioration du niveau de vie - par la santé, l'emploi et les compétences et un environnement plus propre, notamment dans une perspective intergénérationnelle - autant d'éléments dont l'incidence est importante sur la qualité de vie. Les politiques publiques qui favorisent l'accès aux opportunités et aux ressources et mettent en évidence les arbitrages à opérer entre elles et leurs complémentarités devraient être au cœur de cette initiative.

Notre initiative relative à une croissance inclusive procurera un cadre à nos travaux dans ce domaine, qui touchent à de multiples dimensions. Nous entendons déterminer comment définir au mieux le concept d'inclusivité et le mesurer. Pour cela, nous nous inspirerons des travaux déjà bien avancés que nous menons sur la mesure du bien-être. Deuxièmement, nous éclairerons les choix et arbitrages à opérer entre les politiques publiques afin de promouvoir la croissance, tout en veillant à ce que cette croissance 


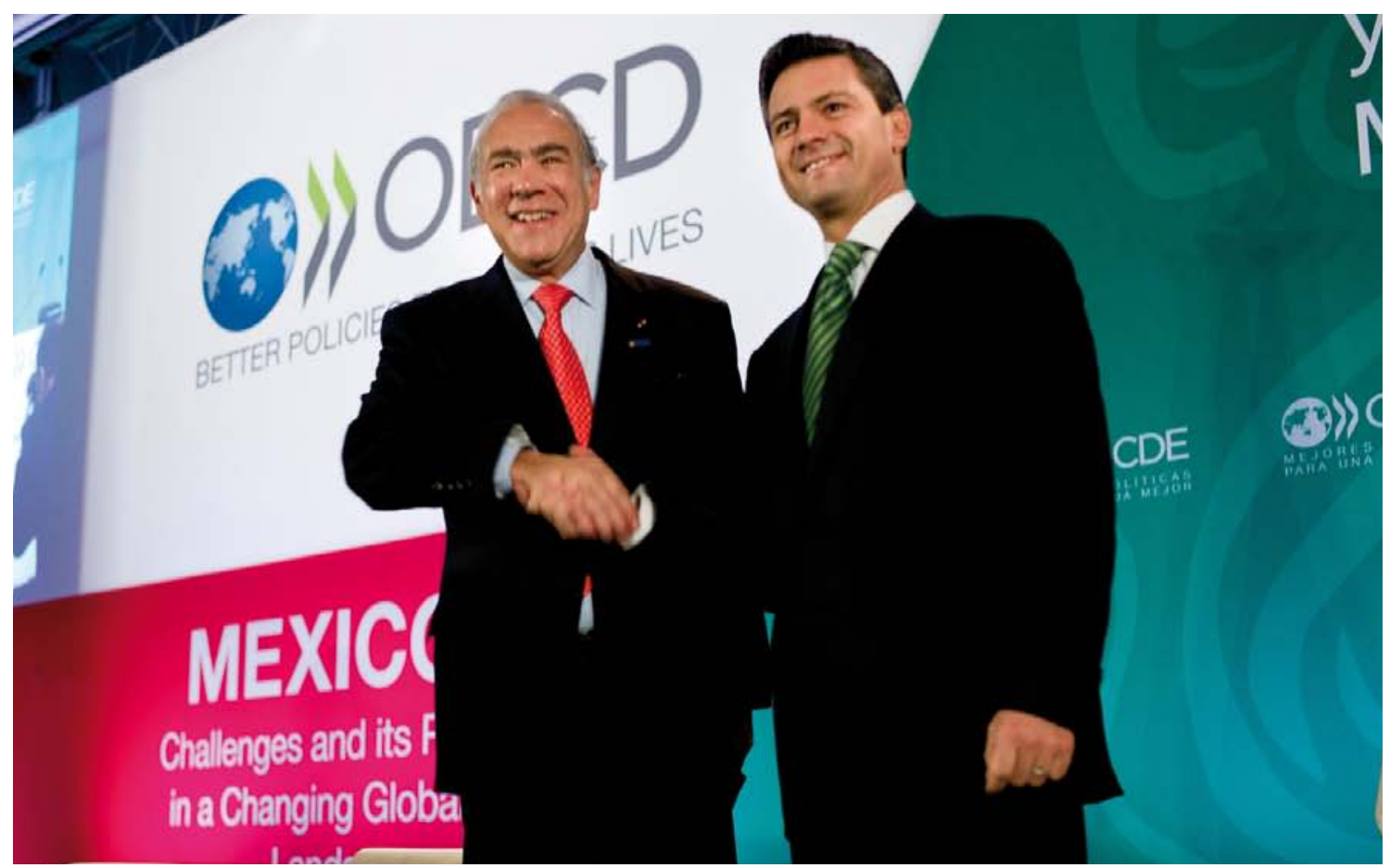

Angel Gurría, Secrétaire général de l'OCDE, et Enrique Peña Nieto, Président élu du Mexique, en octobre 2012

bénéficie à tous. À l'évidence, nos travaux phares sur la croissance, le chômage, les inégalités, l'éducation (PISA) et les compétences (PIAAC, Stratégie sur les compétences), l'entrepreneuriat, l'inclusion financière, les courants migratoires, la santé et le développement sont au cœur de ce programme d'action. Il demeurera prioritaire de donner corps à la Recommandation sur l'égalité homme-femme, qui doit recevoir l'aval des ministres lors de la RCM, et de résorber les inégalités entre les sexes, non seulement pour favoriser la croissance, mais aussi pour promouvoir la justice et l'équité.

\section{L'humain avant tout : emploi, compétences et égalité}

L'OCDE s'est engagée à mobiliser toutes ses compétences et ses atouts pour lutter contre la montée des inégalités, soutenir les populations considérables de personnes sans emploi et favoriser leur retour à l'emploi et améliorer, pour tous, la qualité des emplois. Font partie des priorités fixées :

Plus d'emplois de meilleure qualité - L' ampleur du chômage et du chômage structurel de longue durée, le nombre élevé de travailleurs pauvres, l'instabilité professionnelle et la médiocrité des perspectives de carrière constituent une préoccupation majeure dans de nombreux pays. La poursuite des travaux de l'OCDE sur les réformes structurelles et les politiques actives du marché du travail peut apporter une contribution notable à cet égard. Les travaux relatifs à la promotion de la participation de tous, notamment des jeunes, des travailleurs âgés, des chômeurs de longue durée, des minorités et des populations défavorisées, continueront d'être essentiels. Il importe toutefois d'évaluer la performance du marché du travail non seulement du point de vue de la création d'emplois, mais aussi en termes de qualité des opportunités d'emploi, en tenant compte de plusieurs dimensions. En font partie les perspectives de mobilité professionnelle et de progression salariale qui en découlent sur l'ensemble du cycle de vie.

Le chômage des jeunes - Les jeunes ont été particulièrement touchés par la crise et nombre d'entre eux risquent de se retrouver marginalisés sur le marché du travail. L'OCDE, en première ligne de la lutte contre ce fléau, prodigue des conseils précis à cet égard. En nous inspirant des travaux d'envergure que nous avons réalisés pour nos pays membres et de nos contributions au G20, nous avons mené à bien des analyses en vue :

a) d'évaluer la situation à laquelle les jeunes sont confrontés sur le marché 
du travail, b) de mettre en évidence les principaux obstacles qu'ils doivent surmonter pour obtenir un emploi pérenne ainsi que l'ampleur de l'inadéquation ou de la sousutilisation de leurs compétences, et c) de faire apparaître l'interaction nécessaire des politiques de l'emploi et des politiques de l'éducation qu'il convient d'appliquer. Au cours de l'année à venir, nous œuvrerons à l'élaboration d'un Plan d'action pour l'emploi des jeunes, qui définisse des recommandations concrètes pour un renforcement des systèmes d'enseignement et de formation professionnelle, une meilleure transition vers la vie active, un soutien ciblé en faveur des plus vulnérables et un accès plus facile à la formation et à l'emploi.

Les compétences, ressource incontournable du 21e siècle - Début octobre, nous lancerons nos premières Perspectives sur les compétences qui s'appuieront sur les résultats de l'enquête $\mathrm{OCDE}$ sur les compétences des adultes (PIAAC). Nous publierons en décembre les résultats du prochain cycle du programme PISA et nous accentuerons nos efforts pour étendre les bénéfices de ce programme à un ensemble encore plus large d'économies lors des prochains cycles. En mettant à profit notre Stratégie sur les compétences, lancée à l'occasion de la RCM de 2012, nous coopérons désormais avec les gouvernements des différents pays pour mettre au point une approche intégrée, à l'échelon tant local que national, qui ne se limite pas à examiner comment l'éducation ou le système de formation débouchent sur des compétences, mais étudie aussi de quelle manière les entreprises promeuvent l'acquisition des compétences dont elles ont besoin et les utilisent dans leur processus de production. Compte tenu de l'évolution des exigences du marché du travail concernant les compétences dont il a besoin, les travaux analytiques en cours donneront des indications sur la manière dont les politiques publiques peuvent être mises en œuvre de façon à en maximiser l'impact sur la quantité et la qualité des emplois.

La lutte contre les inégalités - Mettre à profit nos travaux précurseurs sur les inégalités est une priorité essentielle pour l'Organisation. Il s'agit là d'un domaine dans lequel un grand nombre de travaux approfondis doivent encore être menés, en particulier pour atténuer les conséquences sociales des nécessaires ajustements économiques, au nombre desquels : des travaux analysant comment les politiques structurelles qui stimulent la croissance ont une incidence à court, moyen et long termes sur les inégalités ; comment les inégalités ont des répercussions sur la mobilité sociale et la croissance économique ; comment il est possible de parvenir, à moindre coût, à une redistribution des revenus et

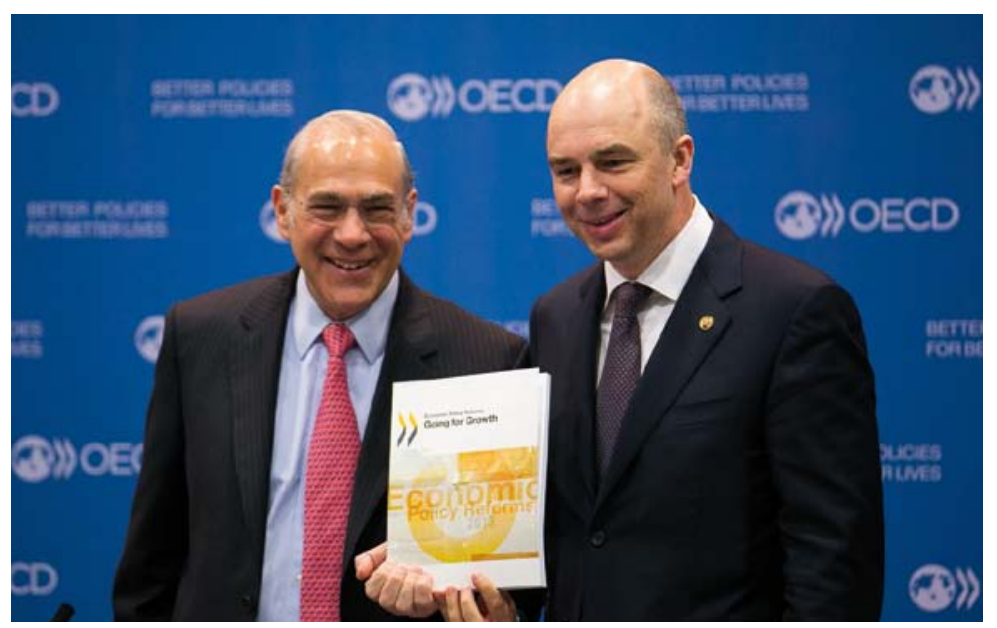

Le Secrétaire général de l'OCDE Angel Gurría et Anton Siluanov, ministre des Finances de la Fédération de Russie, présentent le rapport de l'OCDE Objectif croissance en février 2013 à Moscou quels nouveaux déterminants de la croissance et des inégalités sont apparus. Nous voulons en outre continuer à suivre de près l'évolution des systèmes de protection sociale et des investissements sociaux, et à contrôler les outils que nous recommandons aux gouvernements d'utiliser afin de valoriser le travail, de lutter contre la montée des inégalités et de fournir une couverture contre les risques sociaux qui soit efficace par rapport à son coût. Cela suppose notamment d'intensifier les travaux que nous menons sur le vieillissement, les pensions et la santé.

Nous étudierons de plus près comment mieux préparer la prochaine génération de sorte que les jeunes soient à même d'assumer leur statut de citoyens de leur communauté, de leur pays et du monde. Acquérir les compétences indispensables pour décrocher un emploi ou créer une entreprise et trouver sa place dans un monde plus en plus concurrentiel est le bien-être. Cependant, à l'heure de la mondialisation, les citoyens indispensable pour la prospérité et 
ne peuvent se dispenser des connaissances, de l'attitude et des compétences requises pour pouvoir être actifs au sein et au-delà des frontières de leurs communautés et de leurs régions. Ils doivent en outre comprendre en quoi la vie au sein de leur collectivité et de leur pays a une influence sur les autres nations, et l'impact des événements internationaux sur leur vie quotidienne. Dans ce contexte, nous allons commencer à étudier comment améliorer les systèmes éducatifs afin d'aller au-delà des programmes d'études classiques et de promouvoir la connaissance, les valeurs et les compétences dans des domaines comme la coopération, le respect de la diversité et de l'égalité, la justice sociale et le développement durable. L'accent mis dans le cadre du programme PISA sur l'évaluation des compétences sociales constituera un point de départ.

Les migrations représentent une tendance mondiale de plus en plus importante, ayant des retombées considérables pour la croissance inclusive. Déjà en 2010, les immigrants permanents dans les pays de l'OCDE représentaient plus d'un nouveau venu sur quatre au sein de la population d'âge actif. À condition d'utiliser plus efficacement les compétences sur les marchés du travail, les courants migratoires peuvent contribuer à résorber les déséquilibres démographiques entre les pays frappés par le vieillissement rapide de leur population et les pays émergents ou en développement dont la population est comparativement plus jeune. Nous étudierons par conséquent les obstacles, supposés ou réels, à l'emploi et à l'intégration sociale réels des immigrants que constituent, par exemple, le cadre juridique, les lacunes linguistiques, les diplômes obtenus à l'étranger, l'expérience professionnelle acquise à l'étranger et les discriminations dont ils font l'objet. Nous analyserons en outre l'importance absolue et relative de ces facteurs du point de vue de l'emploi, des qualifications, du décalage entre les emplois et les compétences, ainsi que des salaires, afin d'améliorer la situation des immigrants sur le marché du travail.
Enfin, compte tenu de l'importance grandissante des migrations SudSud, l'OCDE s'intéressera aussi aux moyens de renforcer la capacité des pays en développement à intégrer cette dimension migratoire dans leurs stratégies de développement.

Dans la droite ligne de notre Stratégie pour le développement, nous devons continuer à améliorer les mesures que nous prenons pour répondre aux besoins des pays et régions en fonction de leur cadre institutionnel et de leur stade de développement, tout en renforçant la contribution des politiques menées par les pays membres de l'OCDE au développement. Nous axerons nos travaux sur les enjeux de la Cohérence des politiques au service du développement dans des domaines comme la sécurité alimentaire dans le monde et les flux financiers illicites, et nous formulerons des conseils ciblés qui favoriseront une croissance inclusive, à partir d'un premier ensemble d'examens multidimensionnels par pays en Uruguay, aux Philippines, en

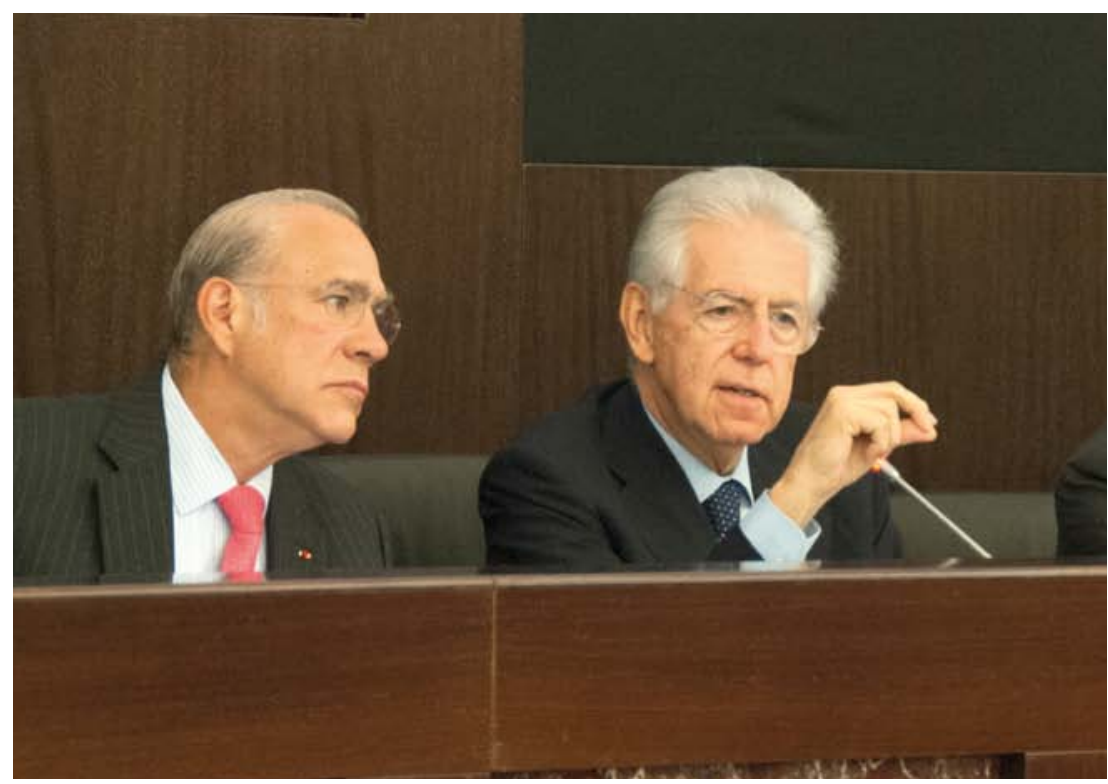

Angel Gurría, Secrétaire général de l'OCDE, et Mario Monti, Président du Conseil italien, à la conference conjointe gouvernement italien-0CDE sur la compétitivité, la croissance et l'emploi à Rome, septembre 2012 
Birmanie et dans d'autres pays. Notre Programme sur la fiscalité et le développement donne déjà des résultats dans des domaines comme les prix de transfert et la gouvernance des incitations fiscales visant à attirer l'investissement. Au cours de l'année à venir, tout en restant focalisés sur l'éradication de l'extrême pauvreté, nous comptons bien contribuer à façonner une nouvelle ère pour le développement mondial, en plaçant l'humain et le bien-être au cœur de nos travaux, en vue d'inscrire le développement dans une perspective de durabilité. À cette fin, nous contribuerons à la définition du cadre dans lequel s'inscriront les objectifs mondiaux après 2015 et nous mettrons à profit les opportunités offertes par le Partenariat de Busan pour une coopération efficace au service du développement, le Dialogue international sur la consolidation de la paix et le renforcement de l'État, le Partenariat statistique au service du développement au XXIe siècle (PARIS21), le G8, le G20, ainsi que par d'autres cadres d'action. Nous poursuivrons la modernisation des dispositifs de suivi et de mesure de l'OCDE afin de prendre en compte les évolutions qui surviennent dans le domaine du financement du développement. Nous continuerons en outre à renforcer le rôle que joue l'OCDE en tant que plateforme de production et de partage des connaissances, notamment par le biais de l'Alliance pour le partage des connaissances et d'autres initiatives. L'essence de la Stratégie pour le développement, notamment l'intégration systématique des considérations de développement dans les travaux de l'OCDE, la cohérence des politiques et le partage des connaissances, doit maintenant présider à l'ensemble des activités menées par l'Organisation. Il convient pour ce faire d'envisager

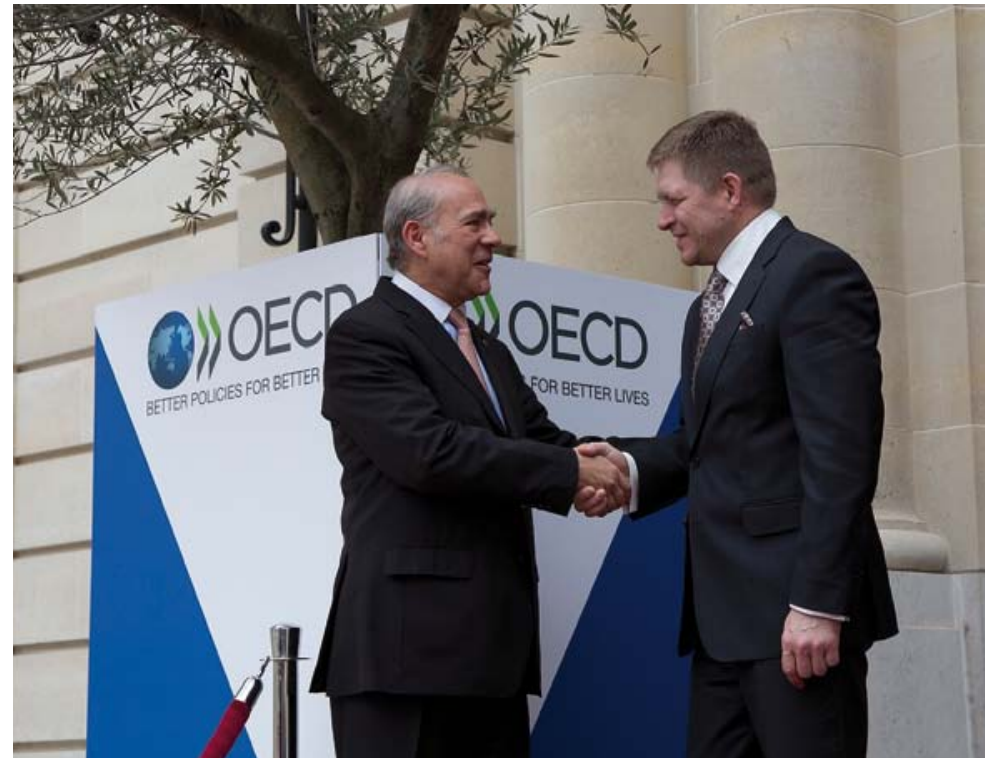

Le Secrétaire général de l'OCDE Angel Gurría accueille Robert Fico, Premier ministre de la République slovaque, à l'OCDE en avril 2013

systématiquement les grands enjeux de l'action publique recensés dans ces Orientations stratégiques dans l'optique de contribuer à l'effort global déployé à l'échelle mondiale.

\section{Interdépendance au service de la croissance}

La structure pluridisciplinaire de l'OCDE est un atout considérable pour faire face à l'interdépendance et à la complexité grandissantes qui caractérisent maintenant l'économie mondiale. L'effort que nous déployons pour préserver l'ouverture des marchés dans les domaines des échanges et de l'investissement est déterminant à cet égard. Nous devons notamment aider les pays à dynamiser ou stimuler leurs économies en luttant contre les risques et en saisissant les opportunités qui découlent de cette interdépendance, en misant tout particulièrement sur de nouvelles sources de croissance. L'exploitation de ce potentiel largement inutilisé accroîtra la productivité, créant ainsi des emplois et stimulant la croissance dans un monde de plus en plus concurrentiel. C'est pourquoi nous voulons, dans l'année qui vient, encourager l'essor de domaines de travail nouveaux et prometteurs comme les actifs intellectuels, les chaînes de valeur mondiales, les échanges en valeur ajoutée et la croissance verte. Nous devons aussi trouver des moyens de mieux mesurer et comprendre les interactions entre ces aspects et nous appuyer sur les travaux que nous menons dans le cadre du G20 pour promouvoir un investissement à long terme efficient et productif, notamment dans les infrastructures.

\section{Plus de connexions et plus de complexité}

La base de données OCDE-OMC sur les échanges en valeur ajoutée a commencé à nous permettre d'appréhender différemment les modes d'échanges, d'investissement et de production dans le monde. Le 
morcellement de la production au plan mondial, favorisé par le progrès technologique, les coûts, l'accès aux ressources et aux marchés et les réformes des politiques commerciales, remet en cause notre manière d'étudier et d'interpréter les statistiques commerciales et, en particulier, les mesures conçues en fonction de ces données. Notre objectif aujourd'hui est d'améliorer encore cette base de données, en l'étendant à un plus grand nombre de pays - notamment à des pays en développement - et de secteurs, et de passer à l'étape suivante en utilisant nos analyses pour élaborer des politiques plus efficaces. Cette nouvelle base de données met déjà échanges et aux investissements internationaux et quels types d'emplois sont ainsi créés. Une autre étape particulièrement complexe consistera à étudier le rôle de l'investissement ainsi que la création et la répartition des revenus qui y sont associées dans le contexte des chaînes de valeur mondiales, notamment le rôle, dans ce cadre, de l'investissement dans le capital intellectuel et des revenus qui en sont tirés (revenus provenant des redevances, de l'octroi de licences et d'autres actifs liés au savoir, par exemple). Ces travaux devraient aussi être élargis à l'étude des flux mondiaux d'investissement - de la même

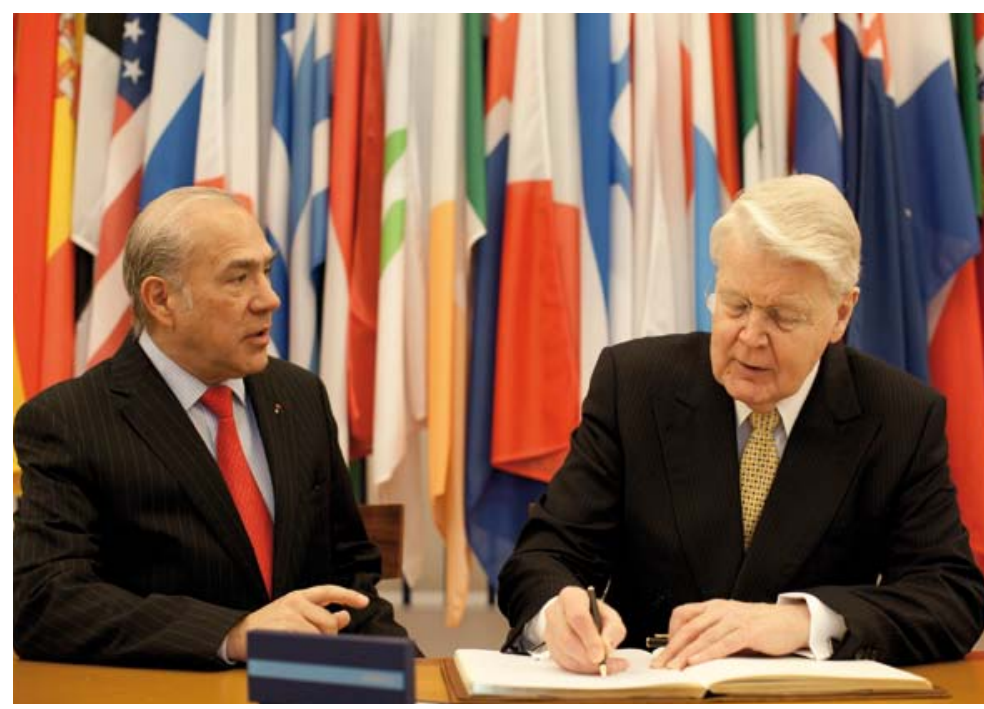

Ólafur Ragnar Grímsson, Président d'Islande, et Angel Gurría, Secrétaire général de l'OCDE, pendant la visite officielle du Président à l'OCDE en février 2013

en évidence l'importance de la facilitation des échanges comme de la libéralisation des services, et nous permettra de mieux quantifier les apports du commerce et le coût du protectionnisme. Nous examinerons aussi en quels points des chaînes de valeur mondiales (CVM) se créent des emplois liés aux façon que l'analyse nous a permis de mieux comprendre les échanges - pour aboutir à l'élaboration d'un cadre d'investissement qui vienne appuyer nos débats dans ce domaine. L'Organisation pourrait en outre renforcer ses travaux sur le rôle des entreprises multinationales dans les échanges, l'investissement et la transmission du cycle économique.

Les initiatives commerciales régionales et multilatérales en cours, que nous examinons actuellement, contribuent à accroître l'interdépendance des plus grandes économies mondiales et des principales régions économiques. Nous ferons encore progresser nos analyses sur le potentiel de croissance que recèlent certaines initiatives récentes, comme l'accord de libre-échange transatlantique entre les États-Unis et l'UE, le Partenariat transpacifique et l'Alliance transpacifique en Amérique latine. De nouveaux travaux doivent être menés sur les canaux par lesquels ces initiatives peuvent promouvoir la croissance des pays qui y participent, ainsi que sur leurs effets attendus sur d'autres régions du monde grâce à l'élargissement des liens commerciaux et financiers.

\section{De nouvelles sources de croissance}

Notre projet horizontal « Nouvelles sources de croissance : les actifs intellectuels ", annoncé à la RCM de 2012, montre que les investissements des entreprises dans les actifs intellectuels sont déterminants pour la croissance de la productivité et les niveaux de vie dans l'avenir. La croissance, en particulier dans les économies avancées, dépendra de plus en plus de gains de productivité fondés sur le savoir. Pour renforcer la croissance à long terme et créer les emplois de demain, nous continuerons d'aider les gouvernements à faire en sorte que les conditions générales, les institutions et les politiques facilitent l'investissement des entreprises dans les actifs intellectuels et l'entrepreneuriat fondé sur la connaissance. La phase 2 
du projet s'articulera autour de trois axes de travail : 1) des travaux complémentaires sur les « données massives ", un actif relativement nouveau, au développement rapide et encore mal connu ; 2 ) l'approfondissement de l'analyse des politiques dans deux domaines qui appellent une attention particulière : la fiscalité et les droits de propriété intellectuelle ; et 3) la poursuite des mesures et des analyses empiriques consacrées aux liens entre les gains de productivité en rapport avec les actifs intellectuels et la croissance, tout particulièrement pour les actifs intellectuels " difficiles à mesurer ", y compris l'investissement dans les données, les études techniques, la formation propre à l'entreprise et le capital organisationnel.

Nous avons commencé à intégrer les conseils sur la croissance verte dans un large éventail de domaines de l'action publique. Nous sommes en train de prendre systématiquement en compte les enseignements de notre Stratégie pour une croissance verte lors de l'élaboration des conseils que nous prodiguons dans les domaines de l'économie, de l'innovation, de la coopération pour le développement, de la finance et du développement urbain aux pays avancés, émergents et en développement. Les futurs investissements stratégiques, en particulier dans les infrastructures, joueront un rôle primordial pour déterminer comment les économies gèreront les tensions qui existent au niveau des ressources et les problèmes de pollution. Des travaux sont en cours pour identifier les principaux obstacles à la prise en compte des considérations environnementales dans les investissements et à un désengagement de la dépendance vis-à-vis des énergies fossiles et des pratiques fortement consommatrices

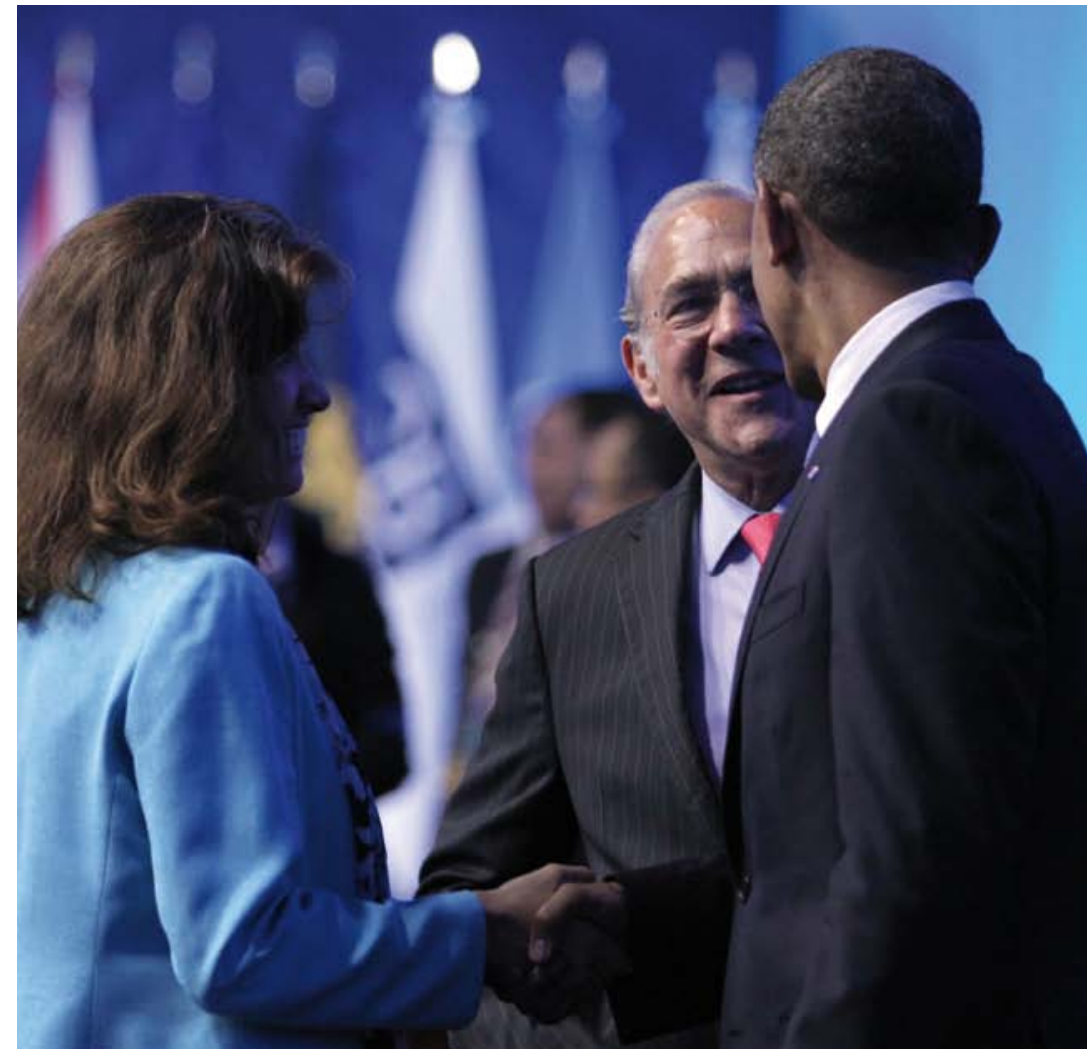

Gabriela Ramos, Sherpa de l'OCDE au G20, Angel Gurría, Secrétaire général de l'OCDE, et le Président des États-Unis Barack Obama au Sommet du G20 de Los Cabos en juin 2012

de ressources. Au cours de l'année qui vient, nous poursuivrons nos travaux sur les indicateurs de la croissance verte et les autres outils de mesure, et nous approfondirons l'analyse des politiques sectorielles, par exemple dans le domaine de l'agriculture et du tourisme. Nous continuerons aussi de renforcer nos capacités de modélisation pour évaluer les coûts de l'inaction face à la dégradation de l'environnement et à la raréfaction des ressources, ainsi que les bénéfices de l'action publique.

L'innovation, notamment dans la $R-D$, reste au cœur de notre quête de nouvelles sources de croissance. La Stratégie pour l'innovation de l'OCDE, lancée en 2010, continue de proposer un large cadre d'actions publiques destinées à dynamiser l'innovation. Depuis qu'elle a été engagée, nous avons utilisé les enseignements de cette initiative pour la Stratégie pour une croissance verte, le projet sur les Actifs intellectuels et les travaux sur le développement, et nous avons lancé de nouveaux travaux dans des domaines comme l'innovation axée sur la demande, l'innovation inclusive, l'innovation dans le secteur public ou encore l'entrepreneuriat innovant. Nous avons par ailleurs entrepris des évaluations en profondeur des politiques d'innovation, assorties de recommandations concrètes pour l'action publique (par exemple à l'intention de la Fédération de Russie, de l'Asie du Sud-Est ou encore de la Suède). Parallèlement à ces travaux, on voit croître 


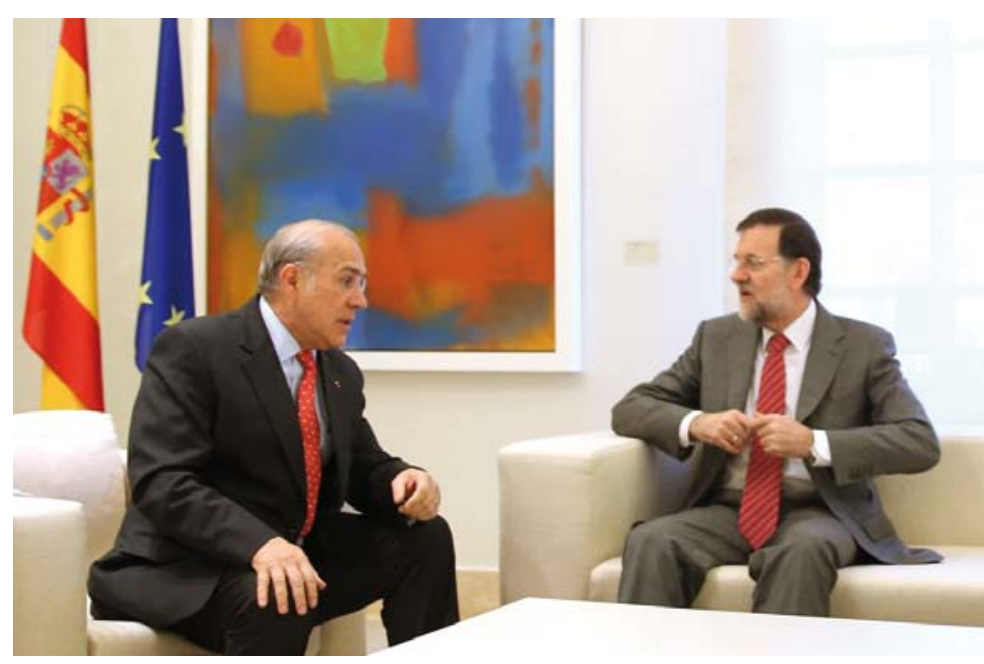

l'intérêt porté à la manière dont les pouvoirs publics pourraient stimuler l'innovation grâce à une " spécialisation intelligente » et à des interventions ciblées visant à favoriser le développement de la science et de la technologie, lequel peut être ensuite converti en innovation et en croissance. À partir des enseignements tirés de ces travaux, nous pourrons reconsidérer et actualiser notre Stratégie pour l'innovation.

Le sport, le tourisme et la culture sont d'importantes sources de bienêtre et d'activité économique. Le manque d'exercice physique joue un grand rôle dans la dégradation de la santé physique et mentale, ce qui nuit au bien-être de la population et coûte aux pays de l'OCDE quelque 160 milliards USD par an en soins de santé. Parallèlement, le secteur du sport génère $2 \%$ environ du PIB mondial (près de 1400 milliards USD en 2011) ${ }^{1}$ et s'est révélé exceptionnellement résistant pendant la crise. Il en va de même pour le secteur de l'art et de la culture. Dans de nombreux pays, ce secteur peut être un important vecteur d'insertion sociale et de bien-être, en contribuant au redressement des économies locales et à l'atténuation des disparités entre régions, mais aussi à l'éducation des jeunes. Le secteur créatif peut apporter une contribution substantielle à la croissance économique. Dans l'Union européenne, les arts et la culture (secteurs culturel et créatif) représentent $3,3 \%$ du PIB et emploient près de 6,7 millions de personnes ${ }^{2}$. Aux États-Unis, près d'un million d'entreprises se consacrent à la création et à la diffusion des arts ${ }^{3}$. À travers ses travaux sur l'Indicateur du vivre mieux, le bien-être et l'inclusivité, l'OCDE peut favoriser la mise en commun des bonnes pratiques et affiner ses analyses de façon à aider les pays à concevoir des politiques permettant de tirer le meilleur parti du sport et de la culture, sur le plan du bien-être aussi bien que de la
Le Secrétaire général Angel Gurría rencontre Mariano Rajoy, Premier ministre espagnol, au Palais de la Moncloa à Madrid, novembre 2012

croissance économique. Elle pourrait par exemple étudier les moyens les plus rationnels en termes de coûts de promouvoir l'activité physique ou les synergies entre le tourisme et l'économie de la création.

\section{La question de la mesure dans un monde interdépendant}

Nombre des initiatives évoquées ci-dessus soulèvent de grandes difficultés en termes de mesure. L'OCDE joue déjà un rôle de premier plan dans la mesure des différentes dimensions du bien-être, de la croissance verte et des échanges en valeur ajoutée, qui met au jour l'interdépendance croissante entre les économies. Cependant, pour contribuer à élaborer des politiques reposant sur des données concrètes dans des domaines aussi divers que les données massives, les actifs intellectuels, l'érosion de la base d'imposition et le transfert de bénéfices ou encore le sport, le tourisme et la culture, et pour approfondir nos travaux consacrés

1. Développer la dimension européenne du sport. Commission européenne, Doc. COM(2011) 12 final. Voir : http://eur-lex.europa.eu/LexUriServ/LexUriServ.do?uri=COM:2011:0012:FIN:FR:PDF

2. Promouvoir les secteurs de la culture et de la création pour favoriser la croissance et l'emploi dans l'Union européenne. Commission européenne. Voir : http://ec.europa.eu/culture/our-policy-development/documents/communication-sept2012.pdf

3. Americans for the Arts, "10 Reasons to Support the Arts", juin 2012. Voir : www.artsusa.org/pdf/get_involved/advocacy/research/2012/10reasons.pdf 
au bien-être et à la croissance verte et inclusive, il sera nécessaire d'investir fortement dans les instruments de mesure et les capacités statistiques de toutes les économies, et mettre davantage l'accent sur l'utilisation des microdonnées. Nous sommes déterminés à collaborer avec nos partenaires nationaux et internationaux pour élaborer un nouvel arsenal de statistiques, d'indicateurs et de cadres comptables, mais aussi pour continuer à améliorer les données classiques pour lesquelles des problèmes de qualité subsistent.

\section{Institutions et gouvernance au service de la croissance}

La crise a mis en relief la nécessité de rétablir la confiance dans les marchés, les gouvernements et les entreprises. La crise appelle des réformes structurelles urgentes qui, pour réussir, nécessitent un leadership politique fort et la confiance des populations. Cependant, force est de constater que les démocraties peinent encore à agir de façon décisive et à obtenir des résultats concrets avec rapidité et efficacité, et ce pour les raisons suivantes:

- un manque d'efficience du système de gouvernance, notamment dans les relations entre l'administration centrale et les autorités locales;

- des blocages liés à l'économie politique et des dysfonctionnements au sein des systèmes démocratiques nationaux;

- des monopoles médiatiques.

\section{Des administrations plus intelligentes, plus transparentes et plus ouvertes}

Dans tous les pays, les responsables publics doivent convaincre leurs citoyens de la légitimité de leur action. Pour que des politiques ambitieuses puissent réussir, il est indispensable d'obtenir l'adhésion et le soutien des parties prenantes. Sans des institutions fortes, avisées et fiables, nos efforts pour mettre en œuvre des politiques meilleures pour une vie meilleure seront compromis. L'OCDE devrait accompagner les efforts déployés pour mettre en place des institutions publiques plus efficaces, plus transparentes et plus ouvertes à tous les niveaux de l'administration, notamment selon les axes d'action suivants :

Instaurer une gouvernance plus avisée et plus efficace en mettant en place une réglementation de meilleure qualité, en optimisant davantage les ressources et en misant sur les nouvelles méthodes et les nouvelles technologies pour faciliter et accompagner la planification et la prise de décisions. Les travaux de l'OCDE en matière réglementaire revêtiront une importance particulière à cet égard. Notre programme intitulé « Mesurer les performances en matière de réglementation " montrera comment les progrès en matière de gouvernance réglementaire peuvent faire baisser les coûts et apporter des avantages concrets aux entreprises et aux particuliers. Il offrira des outils permettant d'identifier les réussites et les échecs, d'améliorer les politiques, programmes et outils réglementaires, et de rendre compte des progrès accomplis. Nous engagerons également des travaux pour faciliter, dans les pays en développement, la mise en œuvre des changements institutionnels dans des domaines comme

Réunion des Sherpas au G20 à Moscou en mars 2013

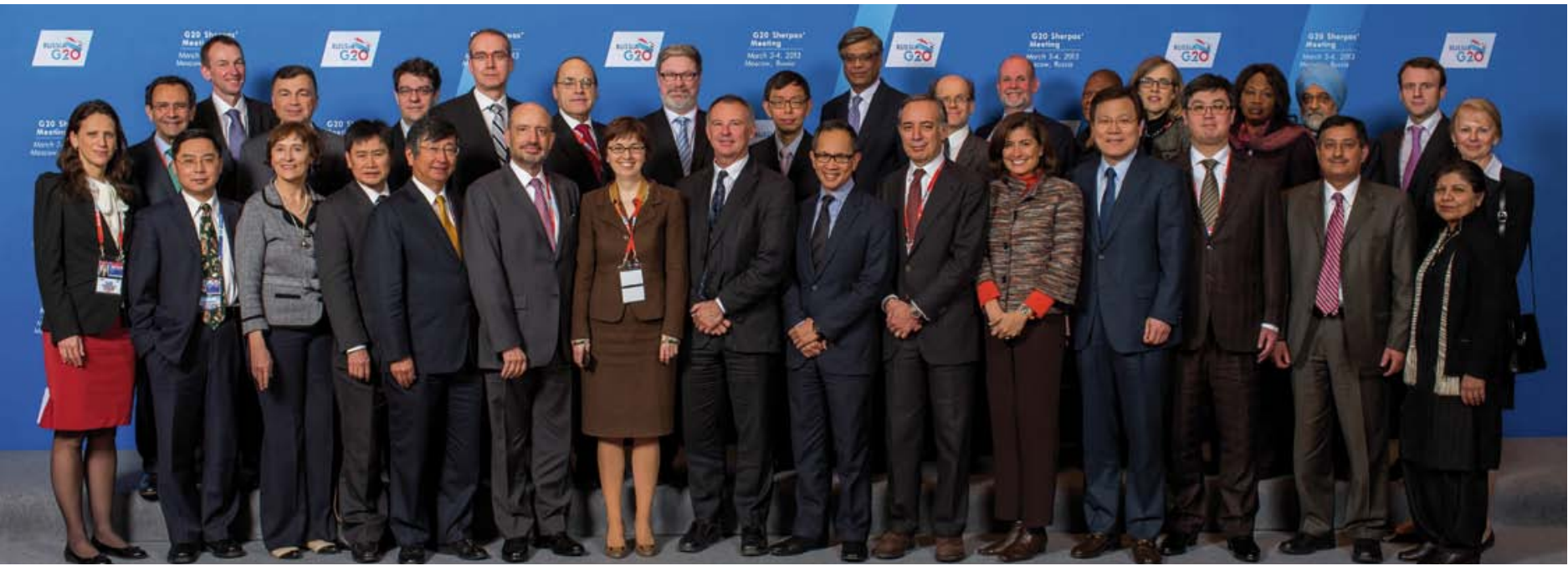




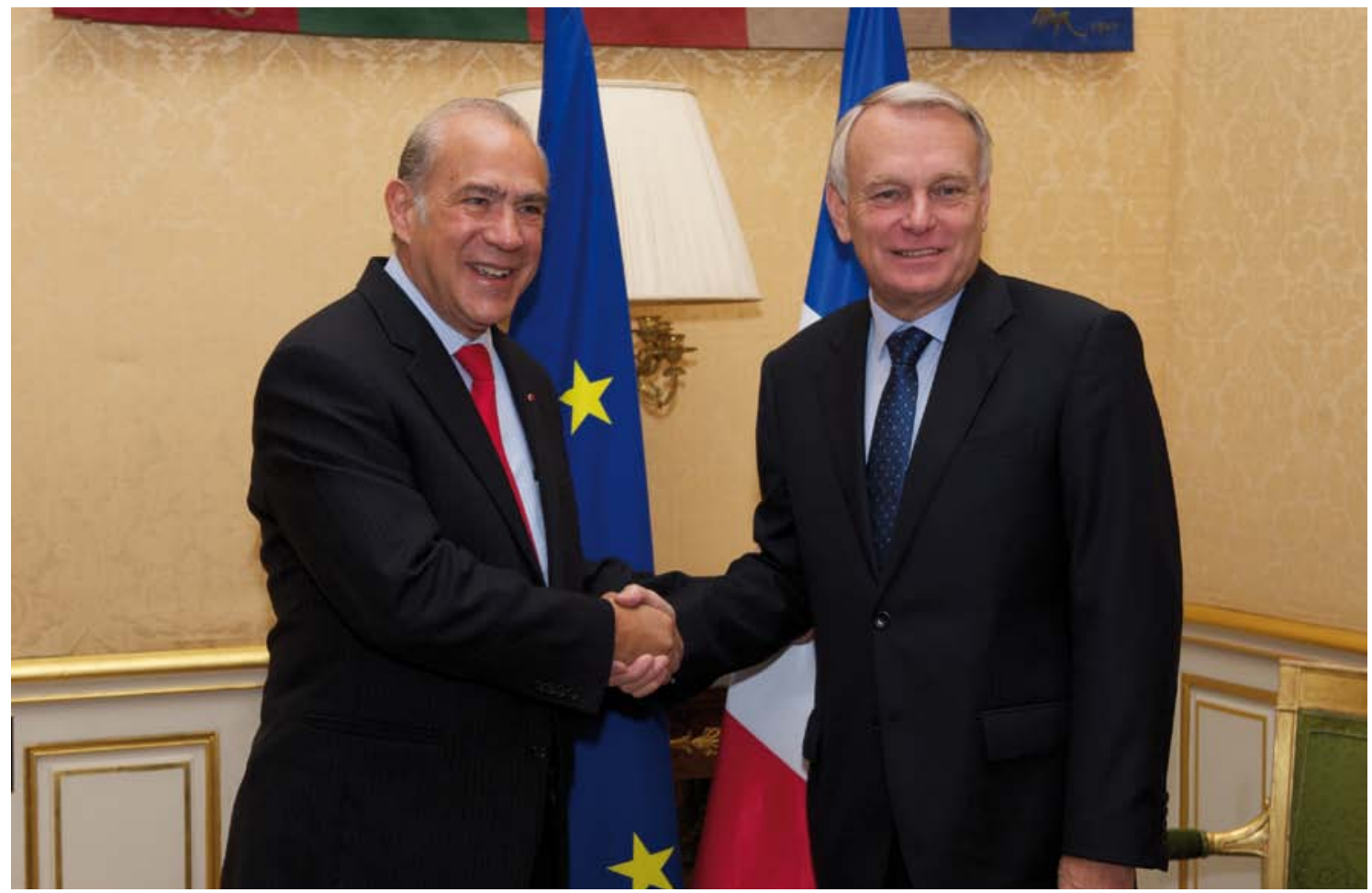

Angel Gurría, Secrétaire général de l’OCDE, et Jean-Marc Ayrault, Premier ministre de France

les marchés publics, la gestion des finances publiques ou encore la responsabilité au niveau national.

Renforcer la transparence et la responsabilité financières permettrait aux gouvernements de montrer clairement qu'ils sont déterminés à regagner la confiance des citoyens. Jusqu'à présent, la plupart des politiques menées en matière de responsabilité et de transparence n'ont pas abordé de front la question des rapports entre argent et politique, ni celle du financement du processus démocratique. Les citoyens attendent de leur gouvernement la preuve que les processus de décision visent bel et bien à servir l'intérêt général et qu'ils soient préservés de toute influence indue. Forte de son expérience en matière de gouvernance, d'intégrité et de transparence, l'OCDE est bien placée pour étudier ces aspects du financement des campagnes électorales et l'impact de ce dernier sur la fixation des priorités politiques et sur les processus de décision. Elle pourrait par exemple s'attacher à déterminer, dans le cadre d'une analyse économique, si le financement privé des campagnes politiques assure une allocation efficiente des ressources, en particulier dans le contexte actuel de crise.

Rendre les administrations plus ouvertes et plus inclusives. À la lumière de notre rapport intitulé The Call for Innovative and Open Government: An Overview of Country Initiatives (2011), qui offre un tour d'horizon des initiatives de différentes administrations nationales en matière d'innovation et d'ouverture, nous allons formuler des recommandations visant à permettre aux administrations de mieux communiquer avec les citoyens et la société civile et de mieux les associer à l'élaboration des politiques. Nous allons, en particulier, aider les administrations à utiliser au mieux les dispositifs d'interactivité existants et à exploiter les autres mécanismes, par exemple les réseaux sociaux ou les données sur l'administration ouverte, de façon à ce que les citoyens puissent contribuer directement à la conception de solutions et à la création de valeur publique.

Il faut aussi adapter les politiques fiscales pour promouvoir des règles du jeu équitables, assurer des recettes adéquates et maintenir des finances publiques saines. Nos travaux sur l'érosion de la base d'imposition et le transfert des bénéfices devraient apporter une 
contribution importante sur ce plan. Il s'agira de réexaminer les règles de fiscalité internationale pour s'assurer qu'il n'y a pas de découplage entre le pays ou territoire où l'activité économique est exercée est celui où le bénéfice est déclaré. Actuellement, un certain nombre de règles permettent aux multinationales de transférer leurs bénéfices là où le régime fiscal leur est favorable, de sorte qu'elles échappent en totalité ou en partie à l'imposition sur les sociétés. Nous allons élaborer un plan d'action complet pour remédier à cet état de fait, et procurer aux pays les informations, les analyses, les bonnes pratiques, les recommandations et les instruments internationaux qui leur permettront de mieux faire face aux pratiques des entreprises du $21^{\mathrm{e}}$ siècle.

\section{Rétablir la confiance dans le secteur privé}

Toutefois, nos travaux en matière de gouvernance concernent aussi les entreprises, qui sont un rouage essentiel de la reprise. Un comportement inadéquat de la part des entreprises peut être à l'origine de gaspillages, d'inefficiences ou de décisions peu avisées et peut

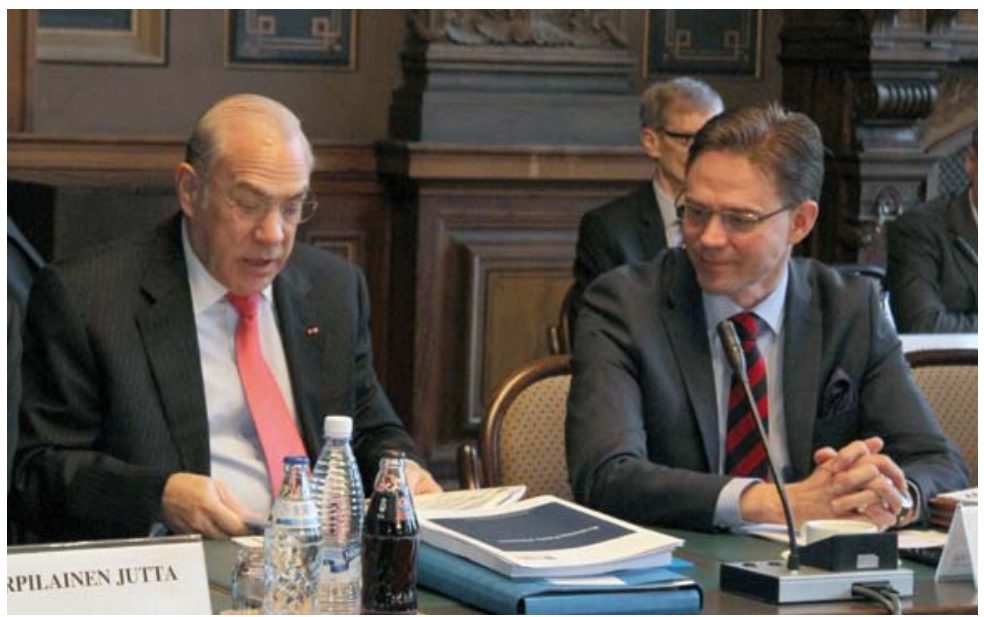

Le Secrétaire général de l'OCDE Angel Gurría et Jyrki Katainen, Premier ministre de Finlande freiner la croissance. Au cours de l'année à venir, l'accent sera mis sur une amélioration du gouvernement d'entreprise axée sur la création de valeur dans les entreprises pour les investisseurs et toutes les parties prenantes. L'OCDE continuera ainsi de faire avancer ses travaux sur le gouvernement d'entreprise, en mettant notamment à profit les travaux en cours consacrés à l'investissement à long terme et au financement des PME. La mise à jour récente des Principes directeurs de l'OCDE à l'intention des entreprises multinationales a aussi constitué une avancée majeure dans le consensus international sur la conduite des entreprises, mais il nous faut désormais les faire mieux connaitre auprès d'autres parties prenantes au niveau international, dispenser des conseils sur la mise en œuvre dans les entreprises et renforcer encore leur mécanisme d'évaluation, qui n'a pas d'équivalent. Nous devons par ailleurs continuer à renforcer notre combat contre la corruption dans les transactions commerciales internationales.

La crise financière a également mis en relief l'existence de graves défaillances au niveau de la régulation du secteur financier. Pour avoir les moyens de détecter précocement les risques d'apparition de bulles, il faudra continuer de surveiller attentivement la dynamique des marchés des actions et des obligations d'entreprise. L'OCDE le dit depuis longtemps : les autorités de réglementation financière doivent s'attaquer aux risques systémiques du modèle économique des grandes banques. Notre préférence va à un système de séparation (société holding non opérationnelle) assorti d'un ratio de levier simple. Les autorités de quelques grandes économies ont commencé à instaurer une séparation entre les activités de banque de détail et les activités de banque d'affaires, qui sont plus risquées. De telles solutions peuvent améliorer la stabilité financière et contribuer à diriger les ressources financières vers l'économie réelle. Toutefois, la méthode suivie varie selon les pays, ce qui ouvre la voie à des stratégies d'arbitrage entre les différentes réglementations. Dans ce contexte, nous poursuivrons nos travaux afin de déterminer les approches les plus efficaces. Comme dans tous les travaux que mène l'Organisation, nous nous appliquerons à coopérer, à coordonner et à partager notre action avec d'autres organisations internationales compétentes intervenant dans ces travaux. L'OCDE doit cependant, pour l'intégrer dans les conseils qu'elle prodigue à ses membres et aux pays partenaires, s'être forgé sa propre opinion, étayée par des données probantes.

Nous poursuivrons également nos efforts pour renforcer la culture financière des individus, tout en améliorant la protection des consommateurs vis-à-vis des institutions financières et des prestataires de services financiers, 
et continuerons nos travaux sur l'accès au financement, ainsi que sur l'efficacité des mécanismes de transmission de la politique monétaire. Comme l'ont souligné les dirigeants des pays du G20, nous sommes très bien placés pour élaborer, dans le domaine de l'éducation financière et de la protection des consommateurs de services financiers, des orientations et des méthodes de mise en œuvre qui aideront les responsables de l'action publique à traduire les politiques en pratiques effectives.

\section{Impact et mise en œuvre au service de la croissance}

En 2012, nous avons nettement accru notre impact sur les programmes des gouvernements nationaux grâce à des conseils concrets sur les politiques à suivre. Nous avons été le partenaire de nos gouvernements au plus haut niveau, œuvrant à leurs côtés pour créer des solutions sur mesure. Au niveau politique, nous avons renforcé nos liens avec les dirigeants et les

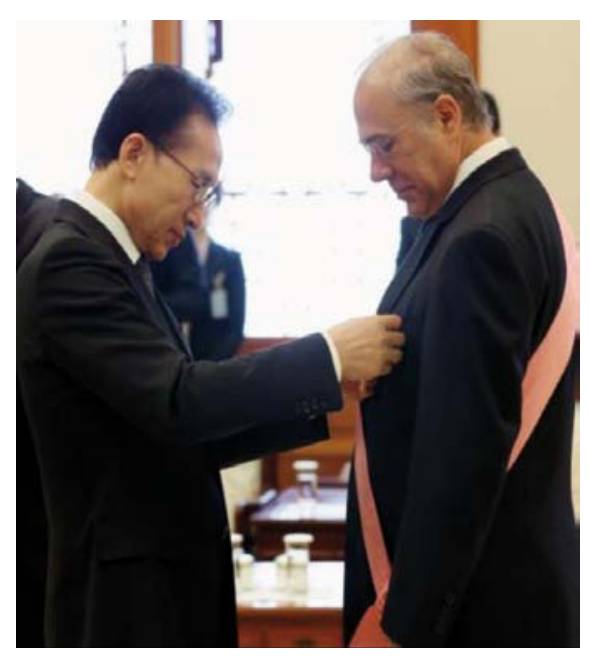

Lee Myung-bak, Président de la République de Corée, décore Angel Gurría, Secrétaire général de l'OCDE, de la médaille Gwanghwan pour service diplomatique en février 2013 grands décideurs. Qu'il s'agisse des conseils fournis à l'Italie et du Séminaire sur la croissance organisé avec le Premier ministre italien, M. Monti ; des discussions sur la question de la cohésion sociale avec la Présidente élue de la Corée, Mme Park Geun-hye; de la visite à l'OCDE de M. Peña Nieto, tout juste élu à la présidence du Mexique, qui est venu accompagné de son équipe de transition, et de notre contribution concrète à son programme de réformes; des discussions approfondies autour de la question des réformes tenues avec le président français M. Hollande, la chancelière allemande, Mme Merkel, et les dirigeants de l'OCDE et d'autres organisations internationales; ou du dialogue avec les Présidents de l'Islande, d'Israël, de la Colombie, du Costa Rica, du Pérou, du Togo et de la Tunisie, et les Premiers ministres de la Finlande et de la Slovaquie, tous ces éléments prouvent le poids politique accru de l'OCDE. Toutefois, il nous faut renforcer encore nos efforts pour développer une approche par pays afin d'en rehausser l'impact.

Nous ambitionnons de renforcer à l'avenir notre rôle de partenaire des administrations nationales et infranationales dans la mise en œuvre des politiques publiques et, pour cela, nous entendons :

Renforcer encore l'impact de nos travaux auprès des gouvernements nationaux, notamment grâce aux brochures de la série Politiques meilleures, à nos rapports sur la compétitivité, à la série Getting it Right et aux examens multidimensionnels par pays. Cela implique de formuler nos conseils sans tarder et de les adapter encore davantage aux besoins spécifiques de chaque pays. Nous allons, par exemple, collaborer avec des régions et États membres de l'Union européenne à la rédaction d'accords de partenariat et de programmes opérationnels avec la Commission européenne pour l'affectation de fonds structurels.

Tenir mieux compte, dans nos travaux, de l'économie politique de la réforme. Nous devons axer davantage nos travaux sur l'étape de la mise en cuure des politiques adoptées, et nous intéresser non seulement à "ce qu'il faut faire ", mais aussi à "la façon de le faire ". Nous devons veiller à ce que nos rapports et nos Comités tiennent compte du contexte, des capacités institutionnelles, à l'échelon tant national qu'infranational, et de la faisabilité politique des réformes que nous préconisons. De plus, nous étudierons la possibilité de formaliser notre partage de connaissances et les conseils que nous dispensons aux pays afin d'appuyer efficacement la mise en $œ u v r e$ des réformes. L'OCDE pourrait, par exemple, renforcer son soutien aux représentants des autorités nationales grâce à des programmes, des ateliers et des formations dans le domaine du renforcement des capacités, à l'image de ce qu'elle propose à l'heure actuelle dans ses Centres fiscaux.

Les personnalités de premier rang étant plus nombreuses à vouloir se rendre à l'OCDE, nous appuyer sur notre expérience pour organiser leurs visites dans le cadre d'un programme spécial consacré à ces personnalités et à leur vision du monde. Ce programme permettra de faire en sorte que la date de la visite soit bien choisie (pour les gouvernements en transition, par exemple) et que son programme soit adapté aux besoins spécifiques des visiteurs (avec, par exemple, des séminaires d'experts, des sessions spéciales du Conseil ou des manifestations ouvertes au public). La phase initiale de ce 
programme ciblera les chefs de gouvernement en visite à l'OCDE, mais nous étudierons la possibilité de l'étendre, à plus long terme, à des représentants éminents du monde des idées, de la sphère économique ou de la société civile.

\section{Un réseau mondial et intégrateur pour l'action publique au service d'une économie internationale plus forte, plus propre et plus juste}

L'OCDE s'est imposée comme un partenaire mondial intégrateur au service de la croissance, s'employant à devenir un « réseau mondial pour l'action publique plus efficace et plus intégrateur ", comme l'y engageait la Vision d'avenir élaborée à l'occasion de son $50^{\mathrm{e}}$ anniversaire. Notre collaboration avec les économies émergentes a été très fructueuse. Ainsi, nous avons mis en place un nouveau Cadre pour la coopération avec l'Indonésie et élargi nos travaux avec les partenaires clés. Nous avons aussi renforcé les initiatives de coopération avec des pays de l'Asie du Sud-Est, du Moyen-Orient et d'Afrique du Nord, de l'Afrique subsaharienne, de l'Amérique latine, des Caraïbes, de l'Europe du Sud-Est et d'Eurasie. Un certain nombre de pays ont fait savoir qu'ils souhaitaient rejoindre l'Organisation, et nous étudions actuellement quelle peut être la réponse à apporter à leur demande d'adhésion. L'année dernière, je me suis rendu à deux reprises en République populaire de Chine, puis en Inde, en Indonésie et en Afrique du Sud. Aux côtés de la Présidence mexicaine du G20, nous avons soutenu les efforts déployés pour faire avancer l'agenda de la croissance verte, nous avons mis sur pied la première réunion des ministres du Commerce du G20

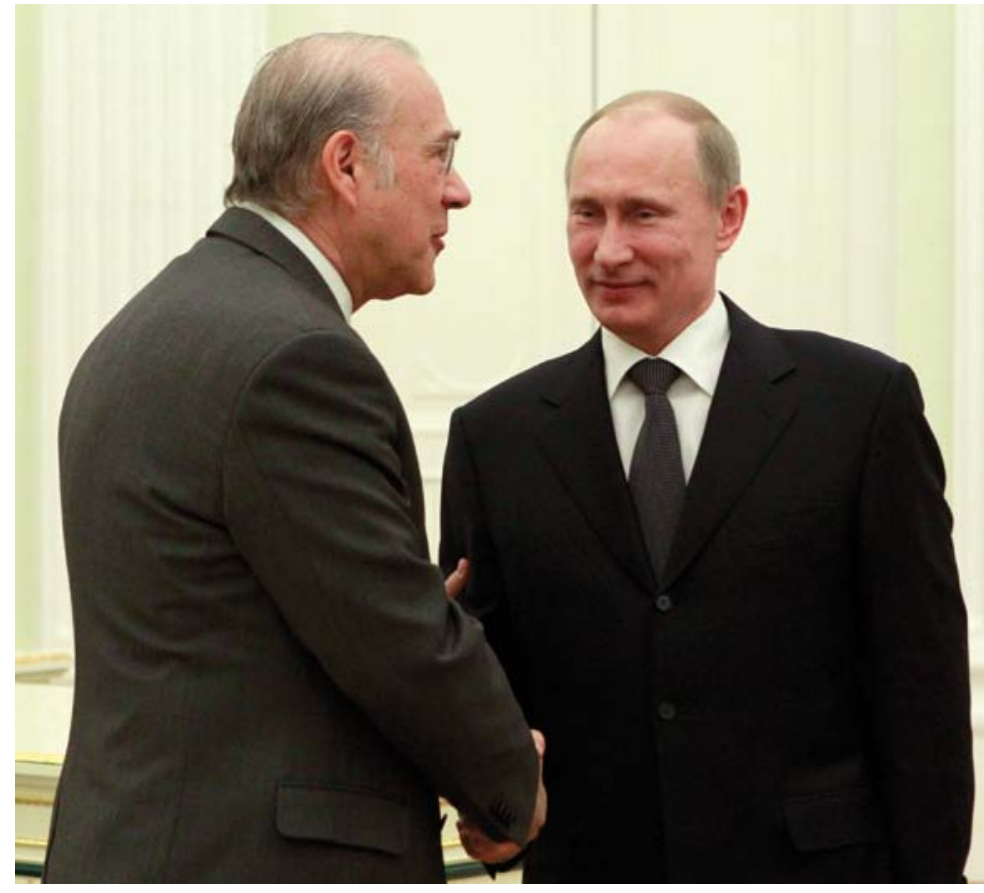

Angel Gurría, Secrétaire général de l'OCDE, et Vladimir Poutine, Président de la Fédération de Russie, à Moscou, le 14 février 2013

jamais organisée et nous avons contribué à la réussite du Sommet du G20 à Los Cabos. Au G8, nous avons renforcé notre présence en apportant une contribution précieuse au Partenariat de Deauville. Par ailleurs, nous avons également commencé à coopérer avec le Royaume-Uni, qui accueillera le Sommet du G8 en 2013, et avec la Fédération de Russie (pour le G20 de 2013 et la G8 de 2014), dont nous soutenons les efforts visant à promouvoir notamment la croissance mondiale et l'emploi.

Pendant les années à venir, nous continuerons à renforcer notre rôle de partenaire mondial intégrateur, en particulier dans les domaines suivants :

Relations avec les partenaires clés - L'année 2012 a vu l'OCDE mettre en place avec succès son premier « Cadre pour un accord de coopération » avec l'Indonésie.
Nous continuerons à rechercher la conclusion d'accords similaires avec d'autres pays partenaires, dans l'optique : de créer des mécanismes permettant d'identifier conjointement les domaines de collaboration prioritaires; de renforcer les échanges d'informations; de favoriser une plus grande coordination grâce à des points de contact établis dans les capitales et dans les ambassades à Paris ; enfin, d'encourager les possibilités de détachement de personnel et d'échanges. Nous nous efforçons par ailleurs d'associer nos partenaires clés aux projets et aux réunions de l'OCDE, en particulier le Groupe NAEC ou encore le Groupe de stratégie globale et, pour la première fois, la réunion à haut niveau du $\mathrm{CAD}$ et la première réunion à haut niveau du Centre de développement. Nous entendons renforcer encore ces efforts d'association des partenaires clés dans les années à venir. 
Initiatives régionales - Au cours de l'année qui vient de s'écouler, nous avons continué à tirer profit des initiatives régionales que nous avons lancées en Amérique latine, en Eurasie, en Europe du Sud-Est, en Afrique subsaharienne et en Asie du Sud-Est. Nous avons également accru de manière très sensible les efforts visant à accompagner la transition et la réforme dans les pays de la région MENA, par le biais du Programme MENA-OCDE et du Partenariat de Deauville. En particulier, l'OCDE a été désignée comme l'une des institutions chargées d'appuyer l'exécution des programmes dans le cadre du Partenariat de Deauville. Cette année, nous continuerons d'approfondir la coopération avec toutes ces régions du monde. Nous élaborerons en particulier un programme régional pour l'Asie du Sud-Est, région déjà identifiée comme une priorité stratégique dans la Résolution de la RCM de 2007. Composé de responsables de l'action publique de la région et de délégués auprès de certains comités spécialisés de l'OCDE, ce programme s'articulerait autour de réseaux thématiques régionaux. Les domaines d'action conjointe prioritaires seraient déterminés dans le cadre d'une approche à l'échelle de l'ensemble de l'administration relayant les perspectives des pays d'Asie du Sud-Est et des pays de l'OCDE, et s'appuyant également sur le rapport annuel intitulé Southeast Asian Economic Outlook. Ce programme aura pour objectif de favoriser les échanges de vues afin de parvenir à une vision commune des grands enjeux de politiques publiques. Il permettra en outre de faciliter l'accès à l'expertise des organes de l'OCDE et l'adhésion à ses instruments.

\section{Adhésion à l'OCDE - Des progrès} significatifs ont été accomplis dans le processus d'adhésion de la Fédération de Russie. Alors que certains comités ont achevé leurs discussions techniques et adopté des avis formels, il faut encore que la Fédération de Russie progresse dans ses travaux pour mener le processus à son terme. L'année à venir, qui verra l'achèvement de la plupart des examens techniques au niveau des comités, sera décisive. Le Conseil sera alors invité à engager des discussions plus politiques. Comme nous y appelait notre
Vision d'avenir élaborée en 2011, nous avons également commencé à examiner les candidatures potentielles de nouveaux pays. Tout nouvel élargissement devrait avoir pour objectif de faire de l'OCDE une organisation encore plus intégratrice, plus pertinente, plus diversifiée et véritablement mondiale.

\section{Gouvernance mondiale - La} participation de l'OCDE aux travaux du G8 et du G20 n'a cessé de prendre de l'ampleur, du point de vue de son impact autant que de sa portée, et l'Organisation apporte aujourd'hui sa contribution à la majorité des questions économiques, sociales et environnementales inscrites à l'ordre du jour de ces deux groupes. Nous continuerons de fournir, en temps opportun et en toute efficacité, des analyses à destination de toutes les enceintes internationales concernées, notamment le Partenariat mondial pour une coopération efficace au service du développement, le Programme de développement pour l'après-2015 et les Objectifs de développement durable, ou encore les négociations sur la CCNUCC. Nous continuerons par ailleurs à collaborer étroitement

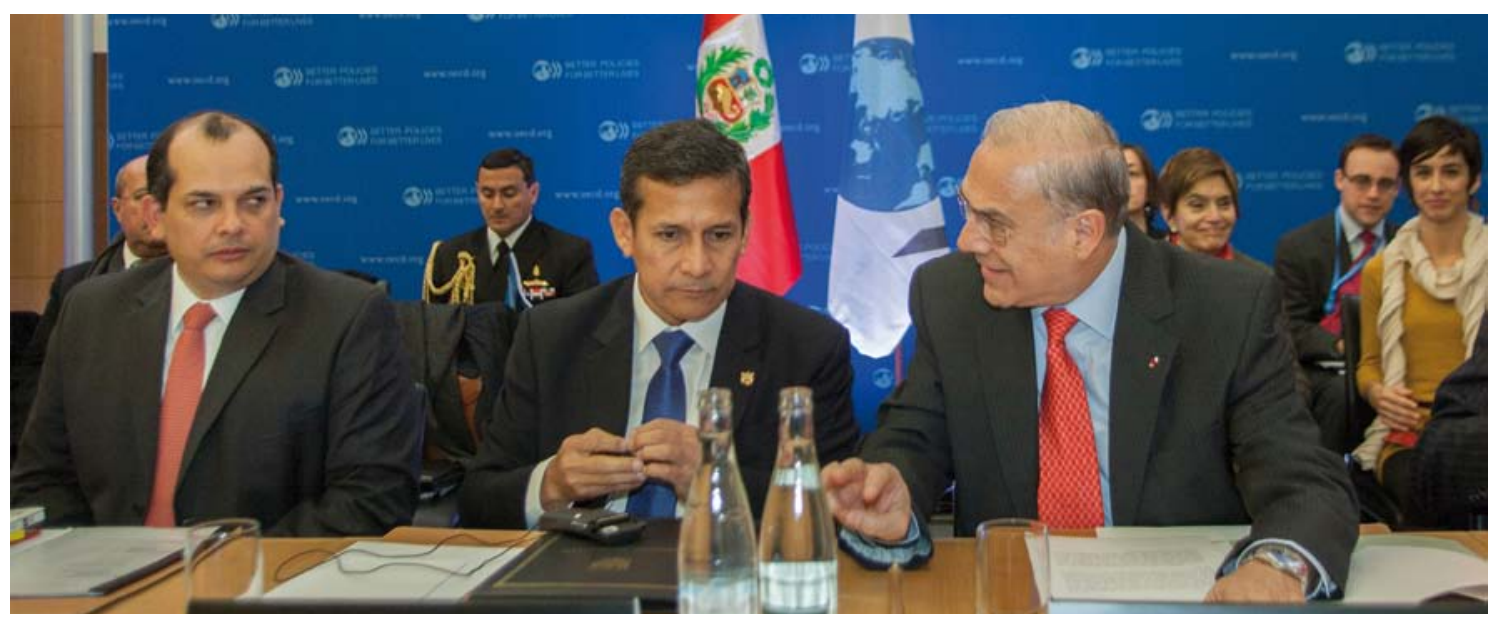

(gauche/droite) Luis Miguel Castilla, ministre de l'Économie et des Finances du Pérou, Ollanta Humala, Président du Pérou et Angel Gurría, Secrétaire général de l'OCDE, pendant la visite du Président péruvien à l'OCDE en novembre 2012 
avec d'autres organisations internationales afin de promouvoir la cohérence des conseils sur l'action publique qui sont dispensés.

Définition de normes à l'échelle mondiale Depuis longtemps, l'OCDE constitue une enceinte où les pays peuvent échanger des expériences et des pratiques exemplaires, mais elle a aussi mis la barre très haut en termes de normes internationales spécifiques, ce qui a beaucoup apporté à nos membres. Pour améliorer encore notre impact et notre pertinence, nous devons agir de manière volontariste pour actualiser, améliorer et compléter notre arsenal de normes et d'instruments, existant ou à venir. Tout d'abord, il nous faut davantage tenir compte des vues des économies émergentes lors de l'élaboration de nos normes, d'une part en les ouvrant à des pays non membres, comme nous l'avons déjà fait par exemple pour le Code de la libération des mouvements de capitaux édicté par l'OCDE, la Convention multilatérale concernant l'assistance administrative mutuelle en matière fiscale, l'Acceptation mutuelle des données pour l'évaluation des produits chimiques, et les Accords sectoriels sur les crédits à l'exportation d'aéronefs civils, d'autre part en tenant compte de l'expérience spécifique des économies émergentes au moment de la mise à jour de ces normes. Nous devons ensuite considérer d'un œil critique nos instruments existants pour évaluer leur pertinence et leur efficacité dans le contexte actuel de l'action publique, apprécier leur impact et en analyser la portée dans les pays extérieurs à l'Organisation. Nous examinerons également quels sont les mécanismes qui nous permettront de mieux aider les pays qui souhaitent se rapprocher des normes et des pratiques exemplaires préconisées par l'OCDE en mettant sur pied des programmes par pays conçus sur mesure.

Besoins qui se font jour en matière de définition de normes à l'échelle mondiale - L'OCDE doit identifier les lacunes et les besoins qui se font jour en matière de normalisation internationale, et étudier où, et selon quelles modalités, l'OCDE pourrait apporter utilement sa contribution. Parmi les domaines dans lesquels nous pourrions envisager dans le futur de définir des normes, on peut citer les statistiques ou encore le financement des partis politiques. En ce qui concerne l'intégrité et, plus généralement, la lutte contre la corruption, nous avons constitué au fil des années nombre d'outils et d'instruments efficaces et nous nous sommes efforcés récemment d'en donner une présentation cohérente grâce à l'initiative CleanGovBiz. Pour accroître notre impact, nous irons plus loin et passerons régulièrement ces instruments en revue, de manière plus systématique, afin de garantir que nous abordons tous les aspects nécessaires de la manière la plus appropriée et la plus efficace.

\section{Servir nos membres sur} la base d'une "stratégie pour l'efficience » et d'une "efficience stratégique »

Nous continuerons à donner corps aux priorités stratégiques qui viennent d'être évoquées en veillant à l'utilisation optimale des ressources de nos membres tout en agissant avec efficience dans un contexte budgétaire difficile. Le Programme de travail et budget 2013-2014 a été élaboré en référence à trois mots d'ordre : « impact, pertinence et intégration ". Si cette triple injonction reste notre raison d'être, « l'efficience, la transparence et la responsabilité " restent au cœur de nos méthodes de travail. Nos services internes continueront d'aider l'OCDE à atteindre ses objectifs stratégiques. À cette fin, nous allons mettre en place une grille systématique destiné à promouvoir des pratiques efficientes dans toute l'Organisation, inspirée des meilleures pratiques des administrations nationales (et infranationales), et la totalité des agents sera associée à cet effort. Nous allons également lancer une initiative de gestion des talents destinée à renforcer la motivation, les performances et l'évolution des agents. Nous redoublerons d'efforts pour recruter des ressortissants qualifiés de pays sous-représentés. Nous continuerons d'évaluer les enseignements tirés de la gestion des projets transversaux afin de faire en sorte que les projets donnent lieu à des résultats concrets et à des recommandations applicables par les pays.

Par ailleurs, nous progressons rapidement en vue de parvenir à la gratuité des données de l'OCDE d'ici la mi-2015 et à la mise en place de notre propre programme de gestion des connaissances et de l'information (KIM) afin de garantir un partage optimal du savoir et de l'expérience accumulés par l'Organisation. En outre, nous sommes en train de préparer une nouvelle série de publications annuelles intitulée OCDE $360^{\circ}$. Cette publication, qui s'inscrit en complément de nos efforts pour développer une approche par pays, passera en revue les principaux axes des travaux que nous menons avec chaque pays membre. Publiée dans différentes langues, elle contribuera à améliorer encore le service que nous rendons à nos membres. 


\section{Activités du Secrétaire général en 2012}

\section{Vue d'ensemble}

En 2012, le Secrétaire général de l'OCDE a continué à mener son programme de travail sur un rythme très intensif, à Paris et à l'étranger, en vue d'accroître la pertinence et la visibilité de l'Organisation. Il s'est avant tout consacré, au cours de cette année, à conseiller les pays membres et à soutenir leurs efforts dans la lutte contre la crise économique et ses répercussions sociales.

Le Secrétaire général a effectué 49 missions à l'étranger au cours de 2012, dans le but de promouvoir les travaux de l'Organisation dans ses pays membres et partenaires. Ces missions ont notamment consisté en la participation aux principaux sommets internationaux qui ont eu lieu au cours de cette année, en particulier le G20, auquel l'OCDE a contribué de manière très active. Le Secrétaire général a rencontré 49 chefs d'État et de gouvernement et 43 dirigeants d'organisations internationales, et participé à 200 réunions avec des ministres, en plus des nombreux contacts informels qu'il a noués à haut niveau en marge des forums internationaux auxquels il a assisté. Il a également rencontré des représentants de haut rang d'autres secteurs de l'administration, des entreprises, des syndicats et de la société civile, ce qui représente un total de près de 1000 réunions bilatérales au cours de l'année.

Dans le cadre de l'exercice de ses attributions courantes de direction et de sa mission stratégique, le Secrétaire général a augmenté, tout comme l'Organisation dans son ensemble, la quantité et la qualité des activités qu'il a menées à Paris en 2012. Le nombre de visites de dirigeants à l'OCDE a continué d'augmenter, et l'OCDE s'est peu à peu imposée comme une étape incontournable du séjour de ces derniers en France. En plus de l'engagement bilatéral du Secrétaire général, le Secrétariat et les délégations ont pu également profiter de la présence de dirigeants à l'OCDE grâce à un programme de conférences accessibles au public, d'allocutions devant les membres du Conseil de l'OCDE et de séminaires qui fait de plus en plus partie intégrante des activités officielles de l'Organisation.

La présence dans les médias et la couverture médiatique sont également restées stables et élevées, suite au niveau record enregistré à l'occasion du 50 anniversaire en 2011, qui a été une année exceptionnelle pour l'Organisation en termes de visibilité. En 2012, le Secrétaire général a été cité dans plus de 1100 articles. La diffusion totale du contenu de l'OCDE a progressé de plus de $20 \%$. Les visites sur le site Internet de l'Organisation ont augmenté de $3 \%$, et la fréquentation de OECD iLibrary s'est améliorée de $40 \%$. La présence dans les médias sociaux a elle aussi fortement progressé.

\section{Activités à l'étranger}

Les 49 missions effectuées à l'étranger se décomposent comme suit : 43 missions dans des pays de l'OCDE, cinq missions dans des pays partenaires clés et une mission dans un autre pays non membre. Cela signifie que le Secrétaire général a effectué en moyenne près d'un déplacement par semaine. Le nombre de ces missions était légèrement supérieur à celui de 2011. Lors de chacune d'entre elles, le Secrétaire général a présenté les travaux de l'OCDE intéressant particulièrement le pays qui l'accueillait et a été régulièrement reçu par les dirigeants et les membres influents des gouvernements. L'objectif principal de ces missions était de promouvoir le rôle consultatif de l'OCDE dans les pays membres, de lancer des travaux menés par l'Organisation, d'accroître la visibilité de l'Organisation dans les principales économies partenaires, et de la représenter au plus haut niveau dans les grands sommets et conférences internationaux. 


\section{Graphique 1. Les missions du Secrétaire général à l'étranger}
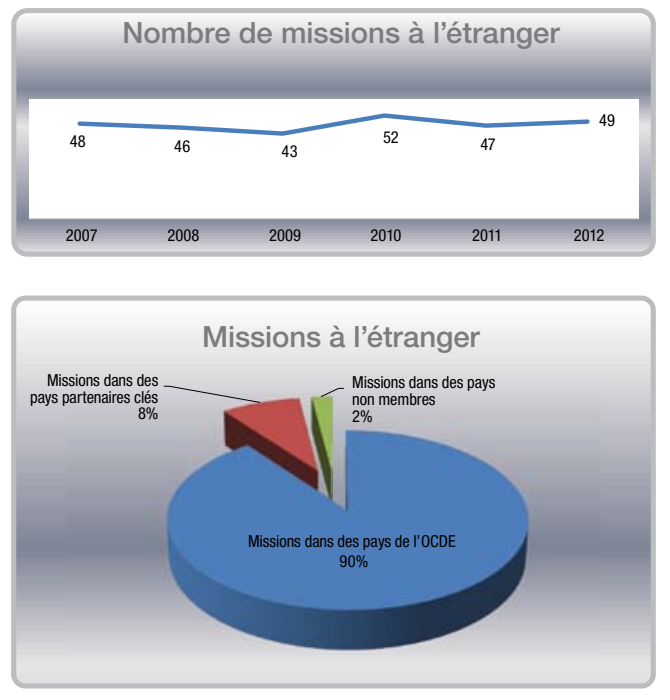

\section{Missions dans des pays membres}

En 2012, le Secrétaire général a effectué 43 missions dans des pays membres, ce qui représente $87 \%$ de l'ensemble de ses déplacements à l'étranger. Au cours de cette année, il s'est rendu dans la moitié des pays membres de l'OCDE. Le pays qu'il a le plus visité est le Mexique, en raison de la Présidence mexicaine du G20. L'OCDE était la seule organisation internationale invitée au plus haut niveau aux réunions ministérielles organisées par la Présidence mexicaine du G20, et notamment aux trois réunions des ministres des Finances et des gouverneurs de banques centrales, à la réunion des ministres du Travail du G20 et à la première réunion des ministres du Commerce du G20, ainsi qu'au Sommet de Los Cabos qui a eu lieu les 18 et 19 juin 2012. Le Secrétaire général s'est également rendu à quatre reprises à Bruxelles au cours de l'année, où il a présenté les études économiques de l'UE et de la zone euro, et a assisté à des réunions à haut niveau avec la Commission et le Parlement européen. Il s'est rendu en Allemagne, en Italie, au Japon, en Espagne, au RoyaumeUni et aux États-Unis à plusieurs reprises, et au Danemark, en Estonie, en Israël, en Corée, en Pologne, en Slovénie, en République slovaque, en Suisse et en Turquie au moins une fois.

Ces missions lui ont permis de renforcer le rôle de conseiller que joue l'OCDE auprès des gouvernements des pays membres pour les aider à faire face aux effets de la crise. Dans certains cas, ces missions ont eu lieu à la demande des gouvernements qui souhaitaient bénéficier d'une aide pour faire avancer leurs programmes de réforme structurelle. Dans d'autres cas, elles ont été l'occasion de présenter des études économiques et d'autres examens thématiques. Le Secrétaire général a notamment présenté en 2012 dans le cadre de ses missions et de ses réunions avec des dirigeants huit brochures de la série Politiques meilleures.

Le Secrétaire général a également représenté l'OCDE et présenté les derniers travaux de l'Organisation à l'occasion de manifestations internationales importantes organisées par des pays membres, dont le Forum économique mondial qui a lieu chaque année à Davos, le Forum mondial de l'eau à Marseille, les réunions FMI/Banque mondiale de Washington et de Tokyo, la conférence annuelle de l'INET à Berlin, le Forum stratégique de Bled et le Sommet ibéroaméricain de Cadiz.

Au cours de ses missions, le Secrétaire général a rencontré les principaux ministres de l'ensemble des pays, ainsi que des dirigeants tels que : les Présidents français, MM. François Hollande et Nicolas Sarkozy ; le Président américain, M. Barack Obama ; le Premier ministre japonais, M. Yoshihiko Noda ; la Chancelière allemande, $\mathrm{M}^{\mathrm{me}}$ Angela Merkel ; le Premier ministre britannique, M. David

\section{Série Politiques meilleures : publications en 2012}

Mexique : Perspectivas OCDE: México Reformas para el Cambio, janvier 2012

Japon : Policies for a revitalisation of Japan, avril 2012

France : Promouvoir la croissance et la cohésion sociale, juin 2012

Mexique : Mejores Políticas para un Desarrollo Incluyente, juillet 2012

Italie : Reviving Growth and Productivity, septembre 2012

France : Redresser la compétitivité, octobre 2012

Allemagne : Addressing the Competitiveness Challenges in Germany and the Euro Area, octobre 2012

Inde : Sustaining High and Inclusive Growth, octobre 2012 
Cameron ; le Président et le Premier ministre italiens, MM. Georgio Napolitano et Mario Monti ; le Président espagnol, M. Mariano Rajoy ; les Présidents mexicains, MM. Enrique Peña Nieto et Felipe Calderón ; le Président turc, M. Abdullah Gül ; le Président et le Premier ministre israéliens, MM. Simon Peres et Benjamin Netanyahu ; le Président et le Premier ministre polonais, MM. Bronislaw Komorowski et Donald Tusk ; le Président et le Premier ministre coréens, MM. Lee Myung-bak et Kim Hwang-sik ; le Président croate, M. Ivo Josipović ; le Président et le Premier ministre slovènes, MM. Danilo Türk et Janez Janša ; la Présidente de la Confédération suisse, Mme Eveline Widmer-Schlumpf ; le Prince Willem-Alexander des PaysBas ; le Premier ministre danois, Mme Helle ThorningSchmidt ; le Premier ministre estonien, M. Andrus Ansip ; le Premier ministre portugais, M. Pedro Passos Coelho; et le Premier ministre slovaque, M. Robert Fico.

Le Secrétaire général s'est réuni régulièrement avec les membres du Parlement des pays dans lesquels il s'est rendu et a assisté au débat annuel de l'Assemblée parlementaire du Conseil de l'Europe à Strasbourg. Il a également rencontré des représentants du secteur privé, des syndicats et de la société civile au cours de ses déplacements à l'étranger. Lors de la plupart de ses missions, il a participé à des manifestations accessibles au public qui lui ont donné l'opportunité de s'adresser à des audiences plus vastes et de diffuser les messages clés de l'OCDE auprès du grand public. Il a notamment prononcé des discours dans des universités, ce qui lui a permis d'entrer en contact avec le monde étudiant. Il a été fait docteur honoris causa par l'Université d'économie de Bratislava, a reçu la Médaille de distinction de l'Institut national de l'administration publique du Mexique et a été admis en tant que membre de l'Académie royale espagnole de sciences économiques et financières.

\section{Missions dans des pays partenaires}

Au cours de 2012, le Secrétaire général a effectué six déplacements dans des pays partenaires, dont cinq dans des partenaires clés : la République populaire de Chine (à deux reprises), le Brésil, l'Inde et l'Indonésie. Afin de soutenir les activités du Secrétaire général, les Secrétaires généraux adjoints se sont également rendus en Fédération de Russie et dans les pays partenaires clés à plusieurs occasions. Au cours de ses visites, le Secrétaire général a présenté des travaux spécifiques menés par l'OCDE sur ces économies, dont des examens économiques, des examens de la réglementation et des brochures de la série Politiques meilleures. Dans le cas de l'Indonésie, le Secrétaire général a conclu un Cadre de coopération avec les autorités du pays. L'Inde a organisé avec succès le $4^{\mathrm{e}}$ Forum mondial de l'OCDE sur les Statistiques, connaissances et politiques, dont le thème était « Mesurer le bien-être pour les politiques de développement ». Comme chaque année, le Secrétaire général a participé au Forum annuel sur le développement de la République populaire de Chine et s'est rendu une nouvelle fois dans le pays en septembre pour inaugurer une conférence à haut niveau sur les chaînes de valeur mondiales au $21^{\mathrm{e}}$ siècle, organisée conjointement par l'OCDE, la CNUCED, l'OMC et le ministère chinois du Commerce. Au Brésil, le Secrétaire général a rejoint les dirigeants au Sommet Rio+20, auquel l'OCDE a contribué activement par le biais de ses travaux sur la croissance verte.

2012 a également été l'année de la première visite effectuée par un Secrétaire général de l'OCDE en Tunisie, à la demande du nouveau gouvernement du pays. Le Secrétaire général a participé à une conférence sur la promotion de l'emploi des jeunes dans la région MENA, organisée conjointement avec la Banque africaine de développement, et s'est réuni avec le Premier ministre et six ministres. Cette visite témoigne clairement de l'engagement croissant de l'OCDE dans la région MENA, en particulier dans le cadre de la participation de l'Organisation au Partenariat de Deauville lancé par le G8.

\section{Activités à Paris}

\section{Rencontres avec des dirigeants et des visiteurs de haut niveau à l'OCDE}

En tirant parti de la dynamique extraordinaire créée par la célébration du 50 anniversaire de l'OCDE en 2011, le Secrétaire général a continué en 2012 à faire de l'OCDE une étape incontournable du séjour à Paris des dirigeants et des parties prenantes concernées. Les dirigeants ont été encore plus nombreux à se rendre à l'OCDE et ces visites se sont réparties sur toute l'année, sans se limiter à la Semaine de l'OCDE, qui a attiré à elle seule six VicePremiers ministres et 59 ministres, Secrétaires d'État ou Vice-ministres de pays membres et partenaires ; parmi les personnalités accueillies figuraient entre autres Sa Majesté la Reine Rania Al-Abdullah de Jordanie, Mme Laura Chinchilla, Présidente du Costa Rica et M. Ali Babacan, Vice-Premier ministre de Turquie.

L'exemple le plus évident de cette présence croissante des dirigeants à l'OCDE a été la réunion entre le Président français, M. François Hollande, et les dirigeants des grandes organisations internationales 
économiques et financières (Fonds monétaire international, Groupe de la Banque mondiale, Organisation mondiale du commerce, Organisation internationale du travail et OCDE), accueillie à l'OCDE à la demande du Président. La visite en octobre de M. Enrique Peña Nieto, Président élu du Mexique, a été elle aussi très utile, car il est venu accompagné de son équipe de transition et, après son discours, a pris part à un séminaire d'une demi-journée avec les experts de l'OCDE sur les principales réformes à envisager avant la mise en place du nouveau gouvernement. D'autres dirigeants ont prononcé des discours devant le Conseil de l'OCDE : M. Ollanta Humala, Président du Pérou; M. Ekmeleddin İhsanoğlu, Secrétaire général de l'Organisation de coopération islamique; Mme María Ángela Holguín, ministre des Affaires étrangères de la Colombie; et Mme Lourdes Aranda, Vice-ministre des Affaires étrangères du Mexique et Sherpa de la Présidence mexicaine du G20. Le Secrétaire général a également rencontré au niveau bilatéral M. Elio Di Rupo, Premier ministre de Belgique; M. Karim Massimov, Premier ministre du Kazakhstan; ainsi que M. Arthème Séléagodji Ahoomey-Zunu, Premier ministre du Togo.

Durant l'année 2012, le Secrétaire général a aussi reçu à Paris 278 hauts fonctionnaires des administrations nationales (soit deux par jour en moyenne, si l'on exclut les week-ends, les congés et les missions à l'étranger), a rencontré 48 parlementaires dans un cadre bilatéral et s'est entretenu à titre individuel avec 33 représentants de haut niveau de la communauté des affaires, des syndicats et de la société civile et 14 universitaires de renom. Il a également reçu des responsables des administrations infranationales, en particulier des maires et des gouverneurs.

\section{Graphique 2. Rencontres bilatérales du Secrétaire général par secteur (2012)}

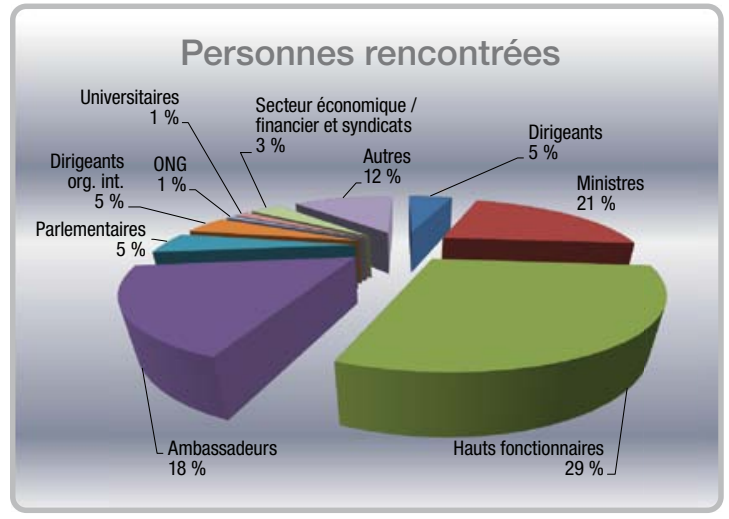

\section{Réunions ordinaires à l'OCDE}

Le Secrétaire général a consacré une grande partie de ses activités en 2012 à ses attributions courantes de direction et à sa mission stratégique au Secrétariat, ainsi qu'à la présidence du Conseil de l'OCDE et aux échanges avec les délégations, en particulier dans le cadre du processus budgétaire 2013-2014. Il a tenu dans l'année 91 réunions bilatérales avec les ambassadeurs auprès de l'OCDE et continué d'accueillir régulièrement des déjeuners avec de petits groupes d'ambassadeurs pour débattre des travaux et des priorités en cours dans un cadre plus personnel et plus détendu. Il a présidé 16 séances du Conseil de l'OCDE, trois réunions des Chefs de délégation de l'OCDE et 13 réunions avec les ambassadeurs assurant la présidence de comités permanents. Le Secrétaire général a également prononcé des allocutions et participé aux événements les plus importants accueillis à l'OCDE, notamment à plusieurs conférences, forums mondiaux et réunions de comités à haut niveau.

\section{Communication et visibilité}

L'un des principaux objectifs de communication de l'OCDE en 2012 était de maintenir le niveau de couverture médiatique atteint en 2011, alors que l'Organisation célébrait son $50^{\mathrm{e}}$ anniversaire. L'intérêt porté aux données, aux analyses et aux conseils de l'OCDE n'a pas fléchi, et s'est même renforcé dans beaucoup de domaines clés grâce à la mise en place de techniques de communication novatrices comme l'insertion de vidéos et de graphiques dans les communiqués de presse ou l'organisation de séminaires en ligne. De plus en plus, le calendrier et la nature des échanges avec la presse sont désormais conçus de façon à s'adapter à l'actualité internationale comme au fonctionnement interne de l'Organisation. Le Secrétaire général a joué un rôle de premier plan dans cette ouverture aux médias.

En 2012, le Secrétaire général a prononcé 220 discours, signé six articles ou tribunes libres, et présenté 29 publications phares, notamment dans les séries de Perspectives. Il a été cité dans plus de 1100 articles et a accordé 165 entretiens au cours de l'année : 91 entretiens audiovisuels (radio, télévision, fil satellite, programmes Internet, diffusion directe) réalisés dans les studios de l'OCDE, 40 interviews à des journaux et des agences de presse de Paris, et 34 interviews à des médias nationaux à l'occasion de missions. 
Graphique 3. Présence dans les médias traditionnels

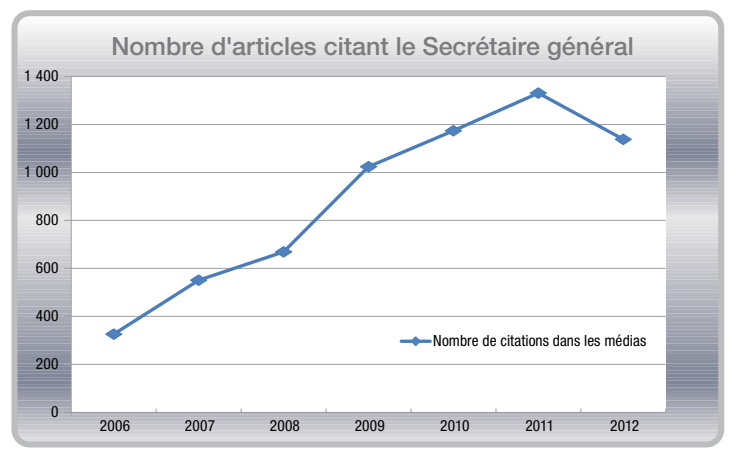

L'Organisation a publié au total 314 titres en 2012, dont 236 rapports et 78 recueils statistiques. À l'occasion de leur parution, 208 communiqués de presse et 67 avis aux médias ont été émis, contre 165 communiqués de presse et 61 avis aux médias en 2011. Une hausse du nombre de notes distribuées aux journalistes a aussi été enregistrée en 2012. Le Secrétaire général a pris part à plus de 30 des 40 conférences de presse environ organisées au cours de l'année, principalement pour le lancement de publications et de rapports de l'OCDE.

Le nombre d'articles consacrés par les médias aux principales publications présentées par le Secrétaire général de l'OCDE a beaucoup augmenté en 2012 : de $44 \%$ pour l'édition du printemps des Perspectives économiques, de $15 \%$ pour l'édition de l'automne, de $91 \%$ pour Regards sur l'éducation et de $52 \%$ pour les Perspectives de l'emploi. En dehors des principales Perspectives et des rapports des séries Regards et Panorama, le Secrétaire général a aussi présenté les études économiques de nombreux pays membres dans leurs capitales respectives, notamment celles de la Suisse, de l'Allemagne, de l'Union européenne et de la zone euro (Bruxelles), de la Pologne, de la Corée, de l'Estonie, de l'Espagne, du Royaume-Uni et de la République slovaque, ainsi que l'étude économique de l'Indonésie, à l'occasion de sa visite à Djakarta.

La présence de l'Organisation sur le web, l'utilisation des nouvelles technologies et la visibilité dans les médias sociaux ont continué de progresser en 2012. La popularité des vidéos de l'OCDE sur Youtube et leur insertion sur d'importants sites d'organes de presse en témoignent tout particulièrement, de même que le renforcement de la présence de l'OCDE sur Twitter et Facebook, qui a triplé en moins de deux ans. L'OCDE a enregistré le trafic le plus élevé sur Twitter à cinq reprises en 2012, à l'occasion du lancement par le Secrétaire général de publications importantes comme les études économiques.

\section{Graphique 4. Réseaux sociaux : " followers " sur Twitter et « j'aime " sur Facebook, 2009-2012}

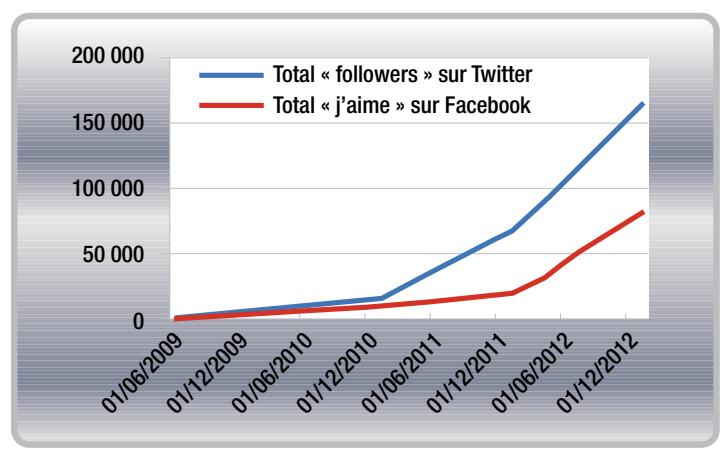

Ce graphique correspond à tous les comptes Facebook et Twitter de l'OCDE. Le nombre de «j'aime » sur Facebook entre 2009 et la mi-2012 a été calculé par extrapolation pour tenir compte de la diversité des dates de création et de la variabilité de la présence sur ces réseaux sociaux.

Du fait de cette présence continue dans les médias, la diffusion totale des contenus de l'OCDE a progressé d'au moins $20 \%$ en 2012 pour atteindre plus de 12 millions de téléchargements, consultations en ligne et exemplaires imprimés (10 millions en 2011). Les exemplaires imprimés sont au nombre de 250000 , le reste correspond à des publications en ligne. La consultation de la plateforme OECD iLibrary a atteint 4,2 millions de sessions ouvertes par 2,7 millions de visiteurs individuels (en hausse de $38 \%$ et $40 \%$ respectivement). Les institutions abonnées à OECD iLibrary sont maintenant plus de 2 500, réparties dans 104 pays.

Graphique 5. Trafic du site web de l'OCDE, 2008-2012

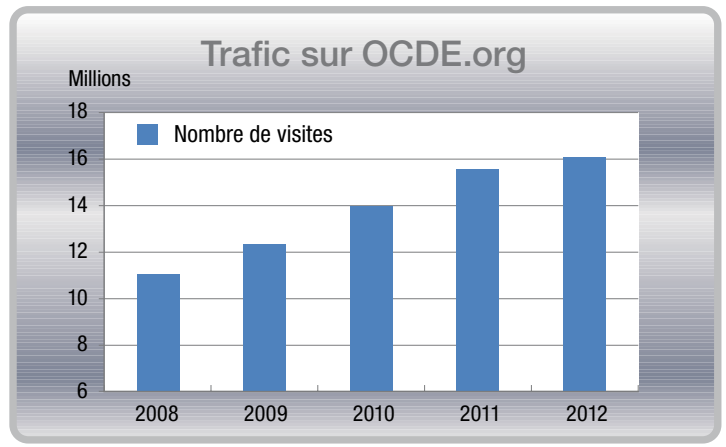

En 2012, l'OCDE a lancé sous la direction du Secrétaire général son programme "Ouverture, accessibilité et gratuité " afin d'appliquer la décision du Conseil de 2011 de mettre à disposition gratuitement toutes les données de la Partie I en 2015. 


\section{Réunion du Conseil au niveau des ministres 2012} Conclusions ministérielles

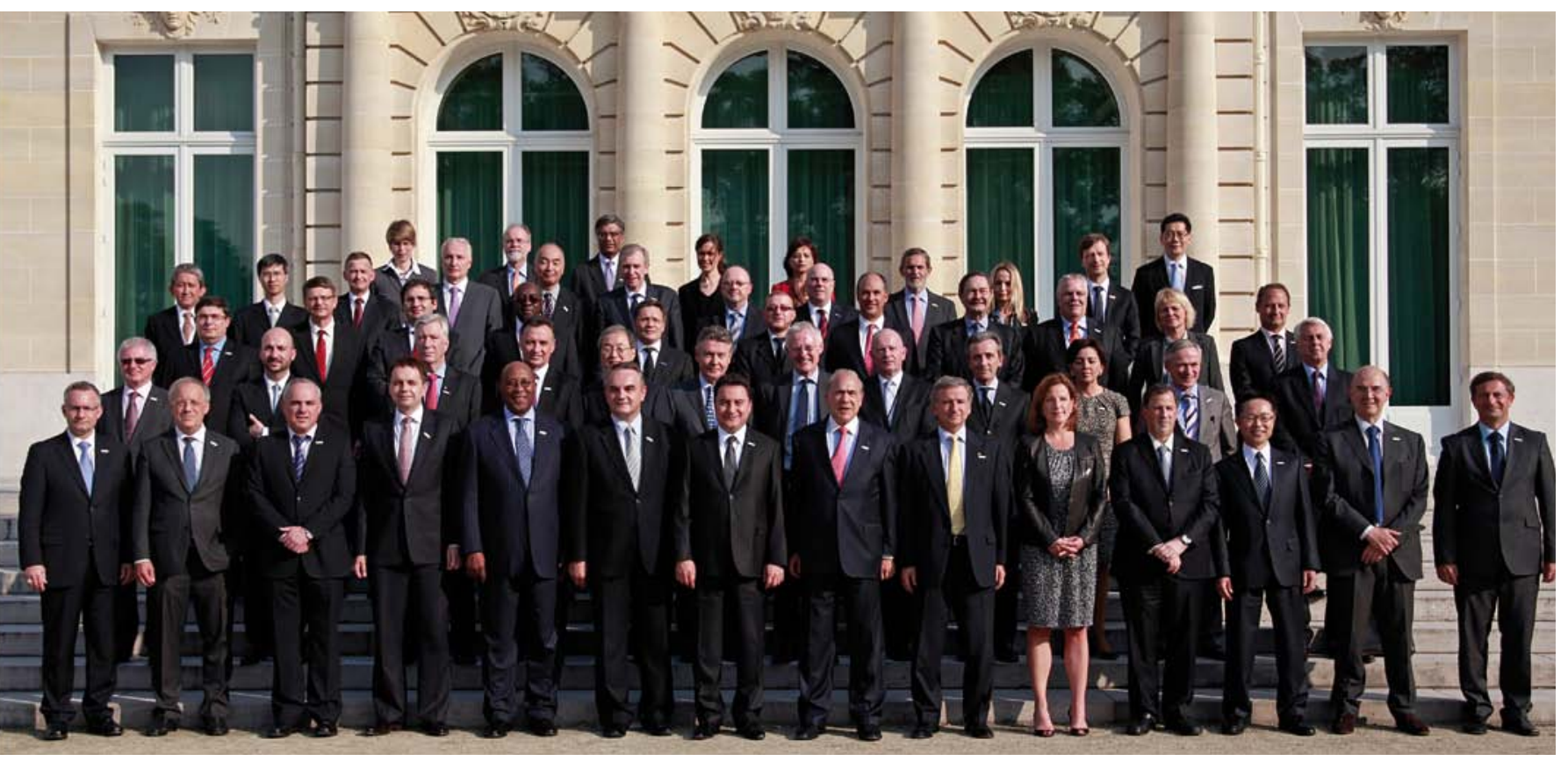

Photo de famille de la Réunion ministérielle de l'OCDE 2012

À l'occasion de la Réunion 2012 du Conseil de l'OCDE au niveau des Ministres, nous nous sommes assemblés sous la Présidence de la Turquie et la Vice-présidence du Chili et de la Pologne. La participation de la Fédération de Russie, dont le processus d'adhésion à l'OCDE est en cours, et de nos partenaires clés Afrique du Sud, Brésil, République populaire de Chine, Inde et Indonésie - ainsi que d'organisations internationales, a grandement enrichi nos débats.
Nous nous félicitons des nets progrès réalisés du point de vue de la mise en œuvre de la Vision d'avenir pour l'OCDE à l'occasion de son $50^{\mathrm{e}}$ anniversaire à laquelle la RCM de 2011 a donné son aval et nous nous engageons collectivement à la traduire en résultats concrets. Nous nous félicitons du rôle que le Secrétaire général a joué en proposant de nouvelles initiatives, notamment ses Orientations stratégiques, et en renforçant la visibilité et l'impact de l'Organisation.
Croissance inclusive et riche en emplois

Compte tenu de la situation économique actuelle, nous sommes fermement attachés à obtenir une croissance inclusive et riche en emplois grâce à des réformes structurelles efficaces, à des politiques budgétaires et sociales responsables et à des mesures propres à stabiliser le secteur financier. Une croissance économique durable est un objectif fondamental et les stratégies futures 
de l'OCDE viseront à promouvoir une croissance verte. Nous soulignons l'importance de donner aux individus et aux entreprises les moyens de surmonter les difficultés économiques actuelles, notamment la tendance inquiétante au creusement des inégalités et les niveaux élevés de chômage observés dans de nombreux pays. Nous encourageons donc à investir davantage dans les compétences humaines et à faire mieux concorder l'offre et la demande de qualifications. Nous insistons sur l'importance d'une participation pleine et entière des femmes à la réussite de nos économies. Nous nous félicitons des nouvelles initiatives de l'OCDE, notamment la Stratégie sur les compétences et l'Initiative pour la parité, ainsi que les travaux en cours sur les inégalités, l'innovation, la croissance verte et les nouvelles sources de croissance, comme les actifs fondés sur le savoir, et sur la mesure du progrès. Ces initiatives sont des sources précieuses d'éclairages et d'orientations et constituent des outils importants pour assurer une croissance génératrice d'emplois et valoriser le capital humain. Nous nous félicitons aussi des travaux menés par l'Organisation en faveur de l'ouverture des marchés, de la sécurité juridique pour l'investissement international et de la conduite responsable des entreprises multinationales, ainsi que de la neutralité concurrentielle entre les entreprises publiques et privées. Nous continuerons de promouvoir la concurrence en tant que moteur d'économies de marché ouvertes et modernes permettant de favoriser l'innovation, d'améliorer l'efficience et d'accroître le bien-être des citoyens.

Nous réitérons notre attachement à un système commercial multilatéral ouvert, à la poursuite de la libéralisation, à la lutte contre le protectionnisme sous toute ses formes et au refus d'adopter des mesures restrictives pour les échanges ou dommageables pour l'investissement. À cette fin, nous approuvons les engagements de statu quo ou de retrait des nouvelles mesures restrictives tels que réaffirmés à l'issue du Sommet du G20 tenu à Cannes; nous chargeons l'OCDE de suivre l'évolution de ces mesures et nous invitons le Comité des échanges, conformément à sa décision, à rédiger un rapport et à organiser un atelier pour évaluer l'impact des mesures liées au commerce. Nous nous félicitons des travaux de l'OCDE sur la relation entre les échanges et l'emploi, les chaînes de valeur mondiales, la mesure du commerce en termes de

étendre à tous les pays qui sont de grands fournisseurs de services, ou qui aspirent à le devenir.

Nous nous engageons à analyser les causes profondes de la crise et à en tirer les enseignements et, au besoin, à actualiser les analyses économiques et les recommandations d'action de l'OCDE. C'est pourquoi nous lançons aujourd'hui l'initiative relative à de "Nouvelles approches face aux défis économiques".

\section{Stratégie pour le développement}

Nous donnons notre aval à la Stratégie de l'OCDE pour le développement, outil essentiel pour renforcer les travaux de

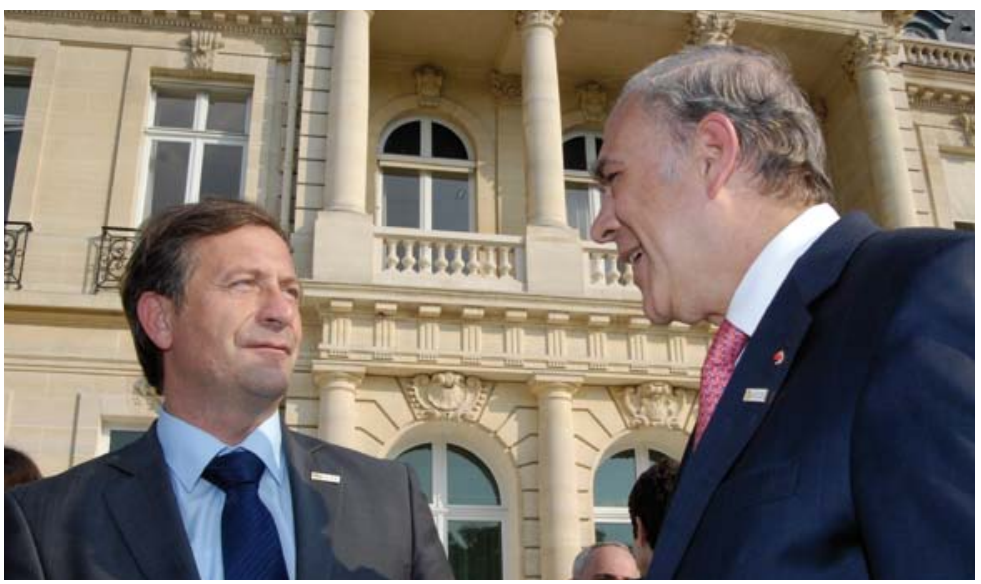

Karl Viktor Erjavec, Vice-Premier ministre et ministre des Affaires étrangères de Slovénie, et Angel Gurría, Secrétaire général de l'OCDE

valeur ajoutée et les restrictions aux échanges de services, de même que les contributions que l'Organisation peut apporter, en coopération avec l'OMC, au programme commercial multilatéral. Nous appelons l'OCDE à accélérer et élargir ses travaux sur l'élaboration d'une base de données et d'un indice sur la restrictivité des échanges de services, et à les
l'Organisation sur le développement, les intégrer et pouvoir les adapter face aux rapides mutations mondiales, de façon que des pays qui en sont à des stades de développement différents puissent contribuer à une croissance durable à l'échelle mondiale. En nous appuyant sur le noyau de compétences spécialisées et 


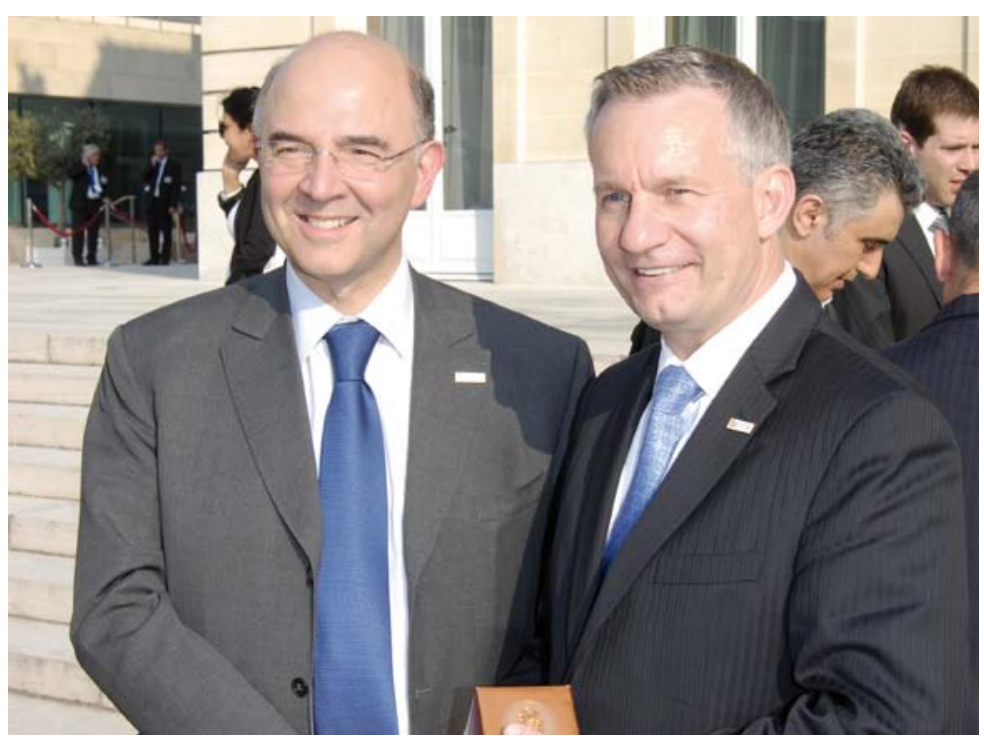

Pierre Moscovici, ministre français de l'Économie, et Ed Fast, ministre canadien du Commerce international

l'expérience de l'Organisation, nous œuvrerons tous ensemble à la mise en œuvre de cette Stratégie. Nous nous efforcerons d'accroître la cohérence des politiques au service du développement et de faciliter le partage des connaissances.

Nous saluons les résultats du Forum de haut niveau sur l'efficacité de l'aide qui s'est tenu à Busan, et nous en soutenons la mise en œuvre, notamment l'accord sur la création du Partenariat mondial pour une coopération efficace au service du développement. Nous intensifierons notre dialogue avec diverses parties prenantes, notamment les économies émergentes.

\section{Réseau mondial pour l'action publique}

Nous notons avec satisfaction l'achèvement des négociations sur l'accession de la Fédération de Russie à l'OMC, la ratification par ce pays de la Convention anti-corruption de l'OCDE et son entrée à l'Agence pour l'énergie nucléaire. Nous espérons que le processus d'adhésion de la Fédération de Russie à l'OCDE se poursuivra conformément à la feuille de route de 2007. Un engagement fort est essentiel sur des questions spécifiques telles que l'environnement, la fiscalité, le gouvernement d'entreprise et le climat des affaires.

Nous affirmons notre volonté de travailler avec chacun de nos partenaires clés (Afrique du Sud, Brésil, République populaire de Chine, Inde et Indonésie) à l'élaboration de nouvelles formes de partenariat et de collaboration. Nous nous efforcerons d'intensifier encore ces relations, dans l'intérêt mutuel, notamment à l'aide d'accordscadres, de la participation plus poussée de ces pays aux travaux des comités et de l'amélioration de la communication.

Nous continuerons d'approfondir notre dialogue avec d'autres pays et régions, dans l'intérêt de tous. Nous travaillerons avec nos partenaires à travers le réseau de l'OCDE pour l'action publique, afin d'atteindre collectivement des objectifs communs tels qu'une croissance durable à l'échelle mondiale. Nous attendons avec intérêt la conclusion rapide des travaux visant à améliorer les règles relatives à la participation de pays partenaires aux activités et organes de l'OCDE. Nous nous félicitons de l'adhésion de nombreux pays aux instruments de l'OCDE. Nous saluons les réformes engagées dans la région MENA et les travaux que mène l'Organisation pour les accompagner, en particulier dans le cadre de l'Initiative MENA-OCDE pour la gouvernance et l'investissement à l'appui du développement, et de sa participation active au Partenariat de Deauville.

Nous apprécions à leur juste mesure les contributions de l'OCDE à la gouvernance économique mondiale, notamment en ce qui concerne la lutte contre la corruption, la fiscalité, l'investissement et la responsabilité sociale des entreprises, l'environnement et le développement durable. Nous saluons le rôle actif que l'OCDE joue auprès de diverses instances telles que le G8 et le G20 dans les domaines où elle possède un avantage comparatif, et la contribution qu'elle apporte face aux défis mondiaux.

\section{Efficience, efficacité et transparence}

Prenant note du souci d'économies qui anime nos administrations nationales respectives, nous encourageons l'OCDE à intensifier ses efforts afin d'améliorer l'efficience, l'efficacité et la transparence et nous nous félicitons de l'engagement de l'Organisation en faveur des données ouvertes et des efforts qu'elle déploie pour faire siennes les meilleures pratiques de gestion. 


\section{Réunion du Conseil de l'OCDE au niveau des ministres 2012 Résumé de la Présidence}

Les ministres des pays de l'OCDE, sous la Présidence de la République de Turquie et la Vice-présidence du Chili et de la Pologne, se sont réunis à Paris les 23 et 24 mai autour du mot d'ordre « Tous à bord : des politiques en faveur d'une croissance inclusive et de l'emploi " afin de définir les stratégies qui devront être adoptées par les pouvoirs publics pour soutenir la reprise après la plus grave crise financière et économique de notre époque, de promouvoir une croissance inclusive et de générer les emplois dont le monde a tant besoin. La RCM 2012 a bénéficié de la participation de la Fédération de Russie, ainsi que de celle de l'Afrique du Sud, du Brésil, de la République populaire de Chine, de l'Inde et de l'Indonésie. Ils ont formulé les Conclusions ministérielles 2012, qui contiennent les orientations des ministres sur les activités en cours et proposées dans le cadre du programme de travail de l'OCDE.
Les ministres ont souligné combien il importe de promouvoir une croissance inclusive et de créer des emplois grâce à des réformes structurelles, de lutter contre le chômage des jeunes, de développer l'investissement à long terme et l'intégration régionale, ainsi que de remettre le système financier en état, de le réformer et de le consolider pour en accroître la résilience. Ils ont également appelé l'OCDE à accorder toute son attention aux enjeux

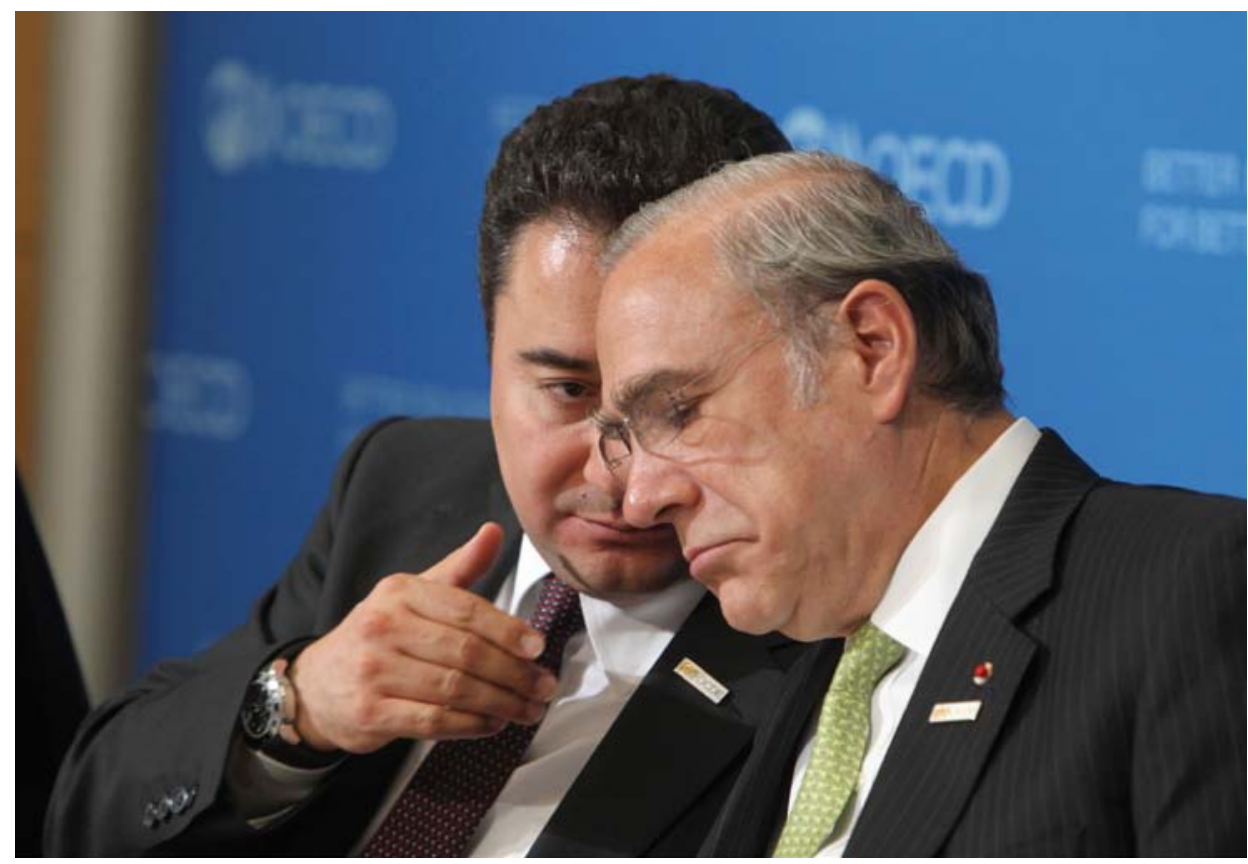

Ali Babacan, Vice-Premier ministre délégué aux Affaires économiques et financières de Turquie, et Angel Gurría, Secrétaire général de l'OCDE 


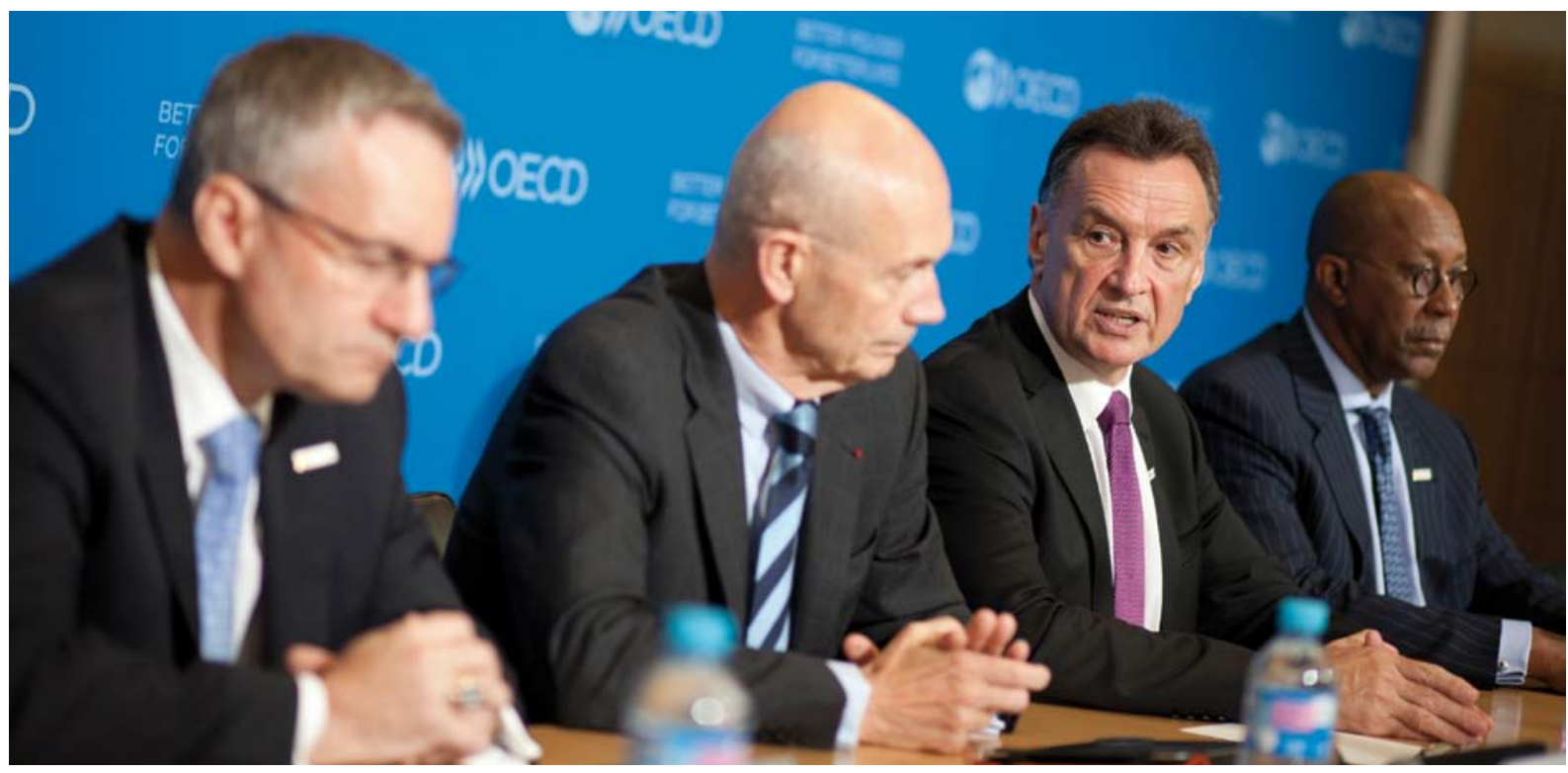

(gauche/droite) Ed Fast, ministre canadien du Commerce international ; Pascal Lamy, Directeur général de l'OMC ; Craig Emerson, ministre du Commerce et de la Compétitivité de l'Australie ; et Ron Kirk, Représentant américain au commerce

du développement et ont insisté sur l'importance des travaux de l'Organisation sur les inégalités.

Ils ont également rappelé la nécessité de communiquer avec les citoyens et de coopérer avec les partenaires sociaux, de coordonner les politiques et d'échanger les meilleures pratiques, ainsi que l'utilité d'une évaluation comparative des performances. Ils ont appelé l'OCDE à conserver un rôle de premier plan à tous ces égards.

Les ministres ont appelé l'OCDE à continuer de fournir des analyses et des avis de première qualité, notamment en généralisant et en renforçant son appel à « penser structurel ", " penser social ", " penser vert » et " penser institutionnel », car ces quatre éléments permettraient d'asseoir les fondations solides qui sont indispensables au rétablissement de la confiance et à la promotion d'une croissance inclusive et riche en emplois. Dans ce contexte, les ministres ont manifesté leur vif soutien à l'initiative qu'ils sont convenus de lancer visant à définir de Nouvelles approches face aux défis économiques. Cette initiative devrait permettre d'analyser les causes profondes de la crise, d'en tirer les enseignements et, au besoin, d'adapter les analyses économiques et les recommandations d'action de l'OCDE. Ils ont appelé à mettre l'accent sur la formulation de recommandations concrètes à l'intention des membres et souligné l'importance, pour ce faire, d'une démarche horizontale. Les ministres ont invité la Fédération de Russie et les partenaires clés à s'impliquer d'emblée dans ce processus. Les travaux de l'OCDE sur la mesure du progrès contribueront à cette entreprise. Ils ont souhaité que ce processus soit inclusif, reste centré sur ses objectifs et que les Comités de l'OCDE, en particulier le Comité de politique économique, y jouent un rôle important. Les modalités exactes du projet et le champ couvert par sa mise en œuvre, qui passera notamment par la création d'un groupe présidé par le Secrétaire général et faisant rapport au Conseil, feront l'objet d'une décision du Conseil qui interviendra après la RCM et avant les congés d'été. Les ministres ont demandé au Conseil de donner régulièrement des orientations à cet égard et à l'Organisation de présenter un rapport d'étape à la prochaine RCM en 2013.

Les ministres ont reconnu que les politiques structurelles, les politiques en faveur d'une croissance verte et les politiques de la science, de la technologie et de l'innovation peuvent se renforcer mutuellement. Ils ont invité l'Organisation à mettre en œuvre les Stratégies pour l'innovation et pour une croissance verte lancées lors de la RCM 2010 et de la RCM 2011 qui a marqué le $50^{e}$ anniversaire de l'Organisation, en prenant systématiquement en compte les recommandations stratégiques formulées à ces occasions dans ses analyses et dans le dialogue sur les politiques à suivre, et en vérifiant les progrès accomplis grâce à des évaluations par les pairs, à l'élaboration plus 
poussée d'indicateurs de la croissance verte et à la collecte de données pertinentes. D'une manière plus générale, les ministres se sont engagés à soutenir des politiques favorables à une croissance et à un développement plus verts, et à apporter leur contribution à la prochaine Conférence des Nations unies sur le développement durable (Rio+20) et aux futures discussions du G20. Par ailleurs, les ministres ont donné leur aval aux conclusions politiques de la réunion du Comité des politiques d'environnement de l'OCDE au niveau ministériel et de la réunion du CAD au niveau des hauts fonctionnaires à l'intention de la Conférence Rio+20. Ils ont également relevé l'importance de l'application de la Stratégie pour une croissance verte, tant au niveau des zones urbaines que des régions, et de la définition d'outils appropriés de gestion des risques. Ils ont en outre salué la publication du rapport intermédiaire Nouvelles sources de croissance : les actifs intellectuels, soulignant que les nouveaux enjeux de l'action publique mis au jour dans ces travaux seront essentiels à la croissance et à l'innovation dans un tout proche avenir. Ils ont appelé l'OCDE à préparer un rapport d'orientation intégré pour la prochaine réunion en 2013.

Les ministres ont examiné la tendance à la montée des inégalités et ont pris acte des défis économiques, sociaux et politiques qui en découlent. Ils ont noté avec intérêt l'analyse menée par l'OCDE sur l'évolution et les moteurs des inégalités, ainsi que sur les moyens d'action propres à les atténuer. Les ministres sont convenus que les mesures d'assainissement des finances publiques et les réformes structurelles en cours devraient tenir compte du contexte social difficile et de la nécessité d'améliorer les perspectives de croissance économique. Ils ont plus particulièrement invité les pays à lutter contre le creusement des inégalités en adoptant des stratégies axées sur la valorisation du travail, l'aide aux revenus des ménages les plus démunis, l'inclusion financière et l'investissement dans le capital humain et l'emploi. Ils ont salué les travaux pionniers réalisés par l'Organisation sur les stratégies de lutte contre le chômage des jeunes. Ils ont pris note des recommandations formulées par l'OCDE sur les réformes des marchés du travail et des produits et sur les systèmes de prélèvements et de prestations, qui visent à assurer une meilleure répartition du revenu sans pour autant vider indûment de leur substance les mesures incitatives, et à déterminer comment les services publics ou les services en nature peuvent contribuer à réduire les inégalités. L'éducation financière et la protection financière des consommateurs jouent également un rôle clé à cet égard et peuvent concourir à la pérennité des systèmes financiers après la crise. Les ministres ont appelé l'OCDE à poursuivre et approfondir les travaux menés dans ces domaines, et à proposer un choix de mesures pour relever ces défis.
Les ministres ont donné leur aval à la Stratégie de l'OCDE sur les compétences, qui encourage les gouvernements à investir dans les compétences et l'éducation afin d'améliorer l'employabilité dans la conjoncture actuelle. Ils ont reconnu l'utilité de la Stratégie pour aider les pays à identifier les atouts et les faiblesses de leurs systèmes nationaux de compétences, à les comparer entre les pays et à élaborer des mesures à l'échelle de l'ensemble de l'administration capables de favoriser l'amélioration des compétences, avec à la clé de meilleurs emplois, au service de la croissance économique et de l'inclusion sociale. Les ministres se sont engagés à mener une réflexion sur les recommandations pour l'action publique formulées dans la Stratégie et à prendre des mesures concrètes pour encourager l'acquisition des compétences nécessaires et assurer une meilleure adéquation entre les compétences disponibles et celles dont le marché du travail a besoin. Les ministres ont reconnu l'intérêt des politiques transnationales dans le domaine des compétences et la nécessité de faire

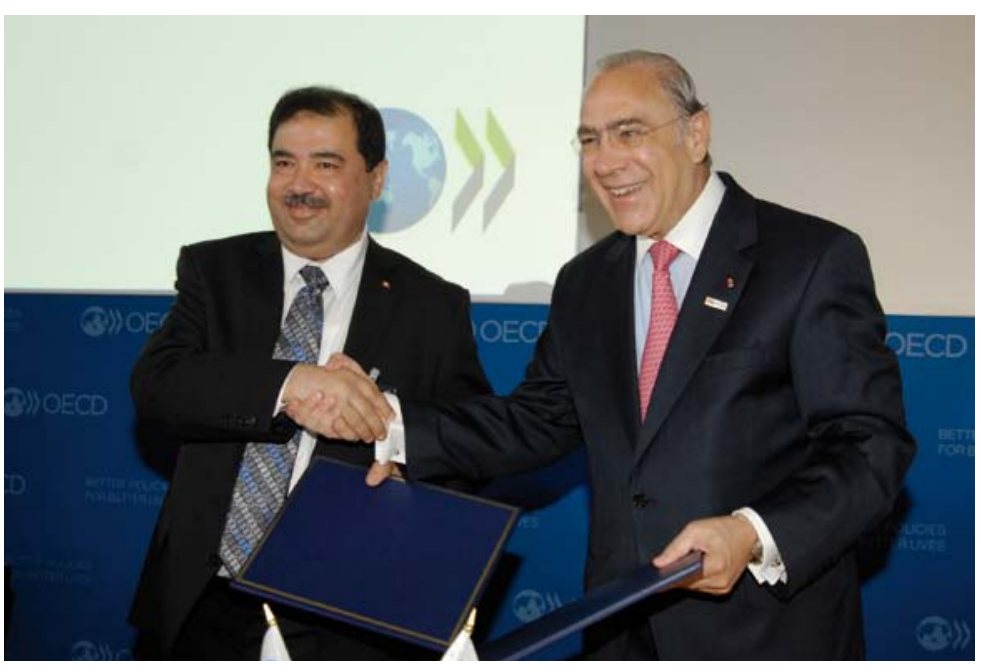

Riadh Bettaieb, ministre de l'Investissement et de la Coopération internationale de Tunisie, et Angel Gurría, Secrétaire général de l'OCDE, lors d'une cérémonie de signature avec la Tunisie et le Maroc 


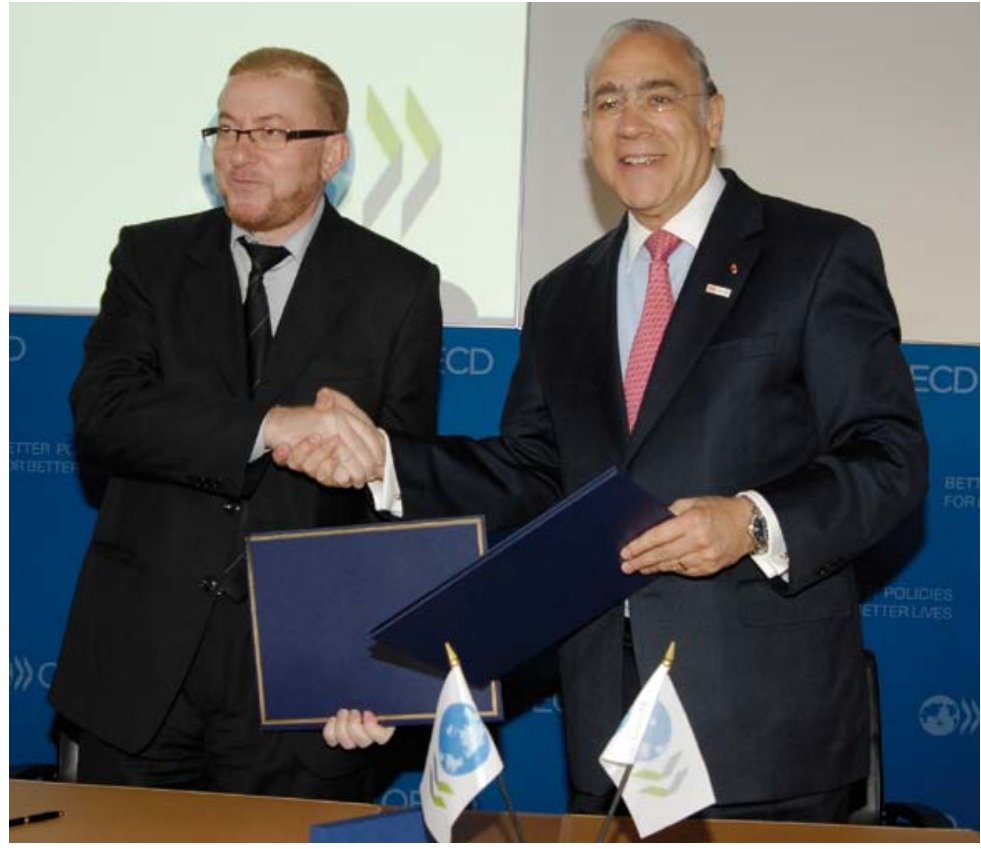

Mohamed Najib Boulif, ministre délégué aux Affaires générales et à la Gouvernance du Maroc, et Angel Gurría, Secrétaire général de l'OCDE, lors d'une cérémonie de signature avec la Tunisie et le Maroc

preuve de souplesse et de réactivité pour répondre aux besoins nouveaux et être efficace dans différents contextes nationaux. Ils ont noté que l'intégration dans la population active de toutes les ressources humaines, y compris des catégories sousreprésentées comme les femmes, les travailleurs migrants et les personnes souffrant d'un handicap, peut considérablement renforcer le vivier de compétences d'une économie.

Les ministres sont convenus de charger l'OCDE d'aider les pays à concevoir des stratégies nationales et locales en faveur des compétences, à les mettre en œuvre et à étendre ces travaux aux pays en développement. Ils se sont félicités de l'occasion exceptionnelle offerte par l'Enquête de l'OCDE sur les compétences des adultes de mesurer les capacités disponibles sur le marché, d'améliorer l'utilisation des compétences au travail et d'évaluer l'influence des compétences sur la situation économique et sociale. Ils ont encouragé l'OCDE à mettre en lumière les principales conclusions de l'Enquête sur les compétences des adultes dans les Perspectives de l'OCDE sur les compétences et attendent avec intérêt les prochaines éditions, qui proposeront des analyses comparatives et par pays s'inspirant des travaux en cours de l'Organisation sur les compétences, et qui contiendront des recommandations pertinentes pour l'action publique. Ils ont également soutenu la création d'un portail électronique interactif dédié aux compétences - baptisé Skills@OECD - qui permettra aux gouvernements, aux chercheurs et aux autres parties prenantes d'accéder aux multiples données et analyses de l'OCDE sur les compétences sous leur forme la plus récente. Les ministres ont pris note de l'intention de la Présidence d'accueillir une réunion informelle des ministres de l'Éducation les 2 et 3 octobre 2013, qui sera consacrée au développement des compétences.

Les ministres ont étudié les inégalités qui persistent entre hommes et femmes dans les domaines de l'éducation, de l'emploi et de l'entrepreneuriat, et ont reconnu que la parité est un enjeu de tous les instants et qu'elle mérite d'être placée plus haut dans les priorités des pouvoirs publics, non seulement au nom de la justice et de l'équité, mais aussi dans le souci de conforter une croissance inclusive, de réduire la pauvreté, d'améliorer la gouvernance, d'encourager l'innovation et de contribuer à atténuer les effets du vieillissement de la population. Ils ont également fait valoir que l'autonomisation économique des femmes est un préalable à la réalisation des $\mathrm{OMD}$ et agit comme un catalyseur et un accélérateur des efforts de développement.

Ils se sont félicités des travaux menés dans le cadre de l'Initiative de l'OCDE pour la parité en vue de mettre en évidence ces disparités et de partager les expériences et bonnes pratiques adoptées pour les corriger. Les ministres se sont accordés sur la nécessité de modifier les comportements à l'égard du rôle des femmes dans la société et dans l'économie, et d'instaurer un environnement de travail adéquat; d'encourager les femmes à être plus présentes dans les domaines scientifiques et techniques; d'offrir des services de garde d'enfants abordables et de qualité et de veiller à valoriser le travail des deux parents ; d'inciter à un partage mieux équilibré du congé parental et du travail à domicile; de définir des objectifs et des mesures visant à accroître la représentation des femmes dans les instances de direction, dans les conseils 
d'administration et dans la politique; et de veiller à l'égalité d'accès au financement des chefs d'entreprise, hommes ou femmes. Les ministres ont appelé l'Organisation à finaliser ces travaux, notamment en complétant la base de données dédiée à l'entrepreneuriat féminin. En s'appuyant sur le rapport final de l'Initiative, ils ont aussi invité les Comités compétents à actualiser, élargir et, le cas échéant, renforcer la Déclaration de l'OCDE sur les politiques en faveur de l'emploi des femmes de 1980 afin de formuler une Recommandation sur l'égalité homme-femme. Ils ont également demandé à l'Organisation de suivre et d'étalonner les avancées réalisées et d'en rendre compte régulièrement.

Les travaux de l'OCDE sur la neutralité concurrentielle ont été accueillis favorablement. L'OCDE a été invitée à développer ces travaux, en collaboration avec la Fédération de Russie et les partenaires clés, et à engager un dialogue sur les politiques devant être suivies par les pays d'origine et les pays bénéficiaires concernant les échanges et les investissements internationaux des entreprises contrôlées par l'État, à favoriser des règles du jeu équitables au niveau mondial, à lutter contre les pratiques protectionnistes et à soutenir la croissance et le développement.

Les ministres ont aussi reconnu la contribution des petites et moyennes entreprises (PME) à la croissance et à l'innovation, à la création d'emplois et à l'inclusion sociale, et ils ont appelé l'Organisation à poursuivre ses travaux dans ce domaine, en particulier sur le financement des PME.

Les ministres ont examiné les conclusions de l'Initiative de collaboration internationale sur le commerce et l'emploi (ICICE) menée sous l'égide de l'OCDE, qui vient à point nommé étayer l'action menée pour favoriser la création d'emplois et pour répartir largement les avantages induits par les échanges commerciaux. Ils ont salué les progrès accomplis à ce jour pour identifier et recenser les obstacles réglementaires au commerce des services. Les ministres se sont félicités des efforts déployés par l'Organisation pour mesurer les échanges en termes de valeur ajoutée et l'ont chargée d'analyser les conséquences, pour l'action des pouvoirs publics, de la multiplication des réseaux de production mondiaux sur l'emploi et sur la croissance dans le protectionnisme et à s'abstenir d'adopter des mesures susceptibles de restreindre les échanges ou de nuire à l'investissement. Certains ministres ont souligné l'importance de l'aboutissement du Cycle de négociations de Doha à l'OMC. À cette fin, les ministres ont approuvé les engagements de statu quo ou de retrait des nouvelles mesures restrictives, tels que réaffirmés par ailleurs à l'issue du Sommet du G20 qui s'est tenu à Cannes l'année dernière. Ils ont chargé l'OCDE de suivre l'évolution de ces mesures, en collaboration continue avec l'OMC et la CNUCED, et invité le Comité des échanges de l'OCDE, conformément à sa décision, à rédiger un rapport

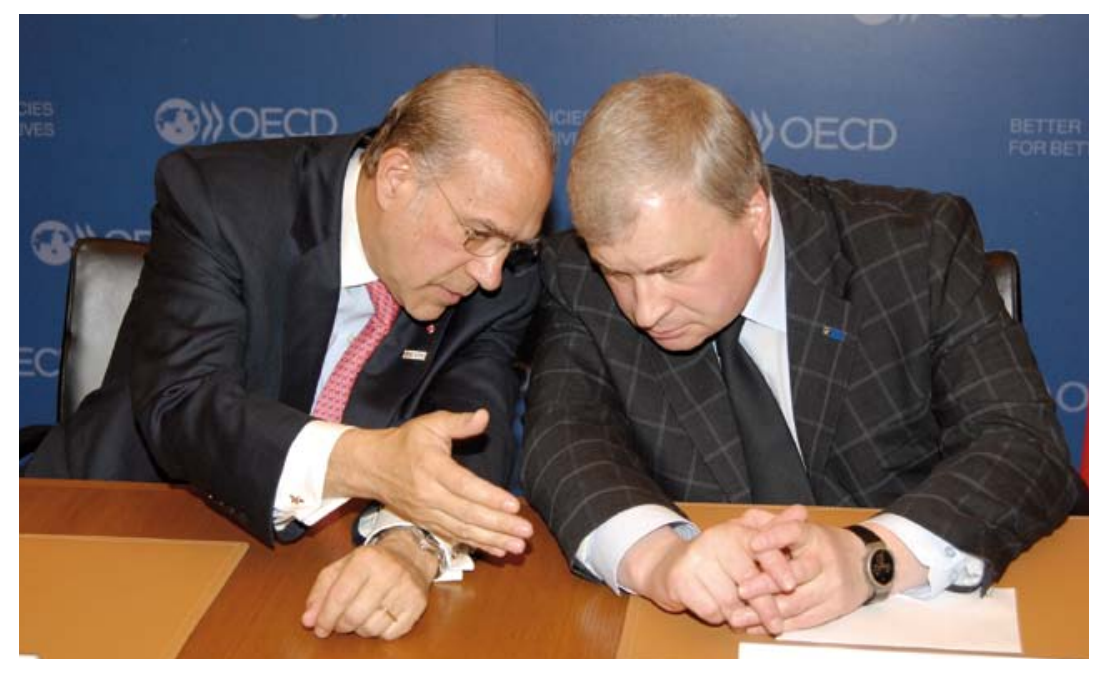

Angel Gurría, Secrétaire général de l'OCDE, et Andrey Ivanovich Denisov, Premier adjoint au ministre des Affaires étrangères de la Fédération de Russie, pendant l'adhésion de la Fédération de Russie à l'Agence pour l'énergie nucléaire (AEN)

les pays de l'OCDE, dans la Fédération de Russie et chez les partenaires clés, dans la perspective de la RCM 2013.

Les ministres ont réaffirmé qu'ils s'engageaient à promouvoir un système commercial multilatéral ouvert, à poursuivre la libéralisation, à lutter contre et à organiser un atelier pour évaluer l'impact des mesures liées au commerce, dans la perspective de leur prochaine réunion en 2013. Ils ont encouragé l'OCDE à accélérer ses travaux consacrés à l'élaboration d'un Indice de restrictivité des échanges de services, à en élargir le champ et à en étendre la portée 


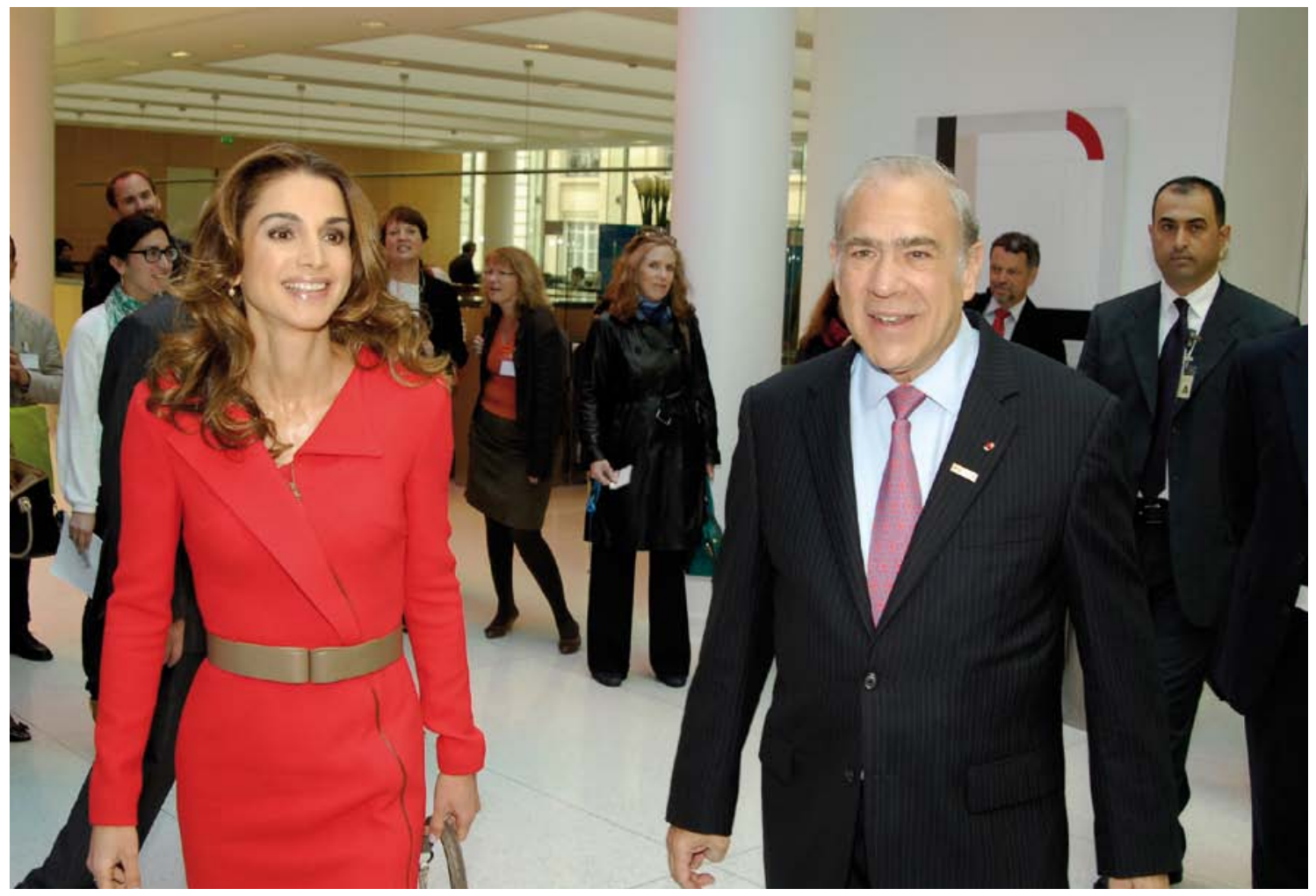

Sa Majesté la Reine Rania Al-Abdullah de Jordanie et Angel Gurría, Secrétaire général de l'OCDE, à leur arrivée au Forum de l'OCDE 2012

au-delà des membres de l'OCDE pour y inclure en priorité la Fédération de Russie et les partenaires clés, les résultats étant attendus pour la RCM de 2014. Les ministres ont reconnu que la facilitation des échanges est l'un des principaux moteurs de la compétitivité et un levier important pour stimuler l'emploi et la croissance, et ont invité l'OCDE à mobiliser ses compétences, notamment dans le domaine de l'aide pour le commerce. Les ministres ont également encouragé l'OCDE à conserver le rôle prééminent qu'elle joue pour promouvoir l'ouverture des marchés, la sécurité juridique de l'investissement international et une conduite responsable des entreprises multinationales, en coopération étroite avec la Fédération de Russie et les partenaires clés.
Les ministres ont réaffirmé leur engagement à l'égard du développement mondial et donné leur aval à la Stratégie de l'OCDE pour le développement, outil essentiel propre à assurer que les travaux de l'Organisation dans ce domaine soient renforcés, intégrés et aptes à répondre à l'évolution rapide de la réalité internationale, où des pays à différents stades de développement peuvent contribuer à une croissance durable, partout dans le monde. Les ministres se sont félicités que l'Organisation suive une approche du développement plus globale et plus inclusive, intensifie ses travaux sur la cohérence des politiques au service du développement et renforce encore sa contribution aux processus mondiaux et à l'architecture du développement. Les ministres ont appelé l'Organisation à intensifier son dialogue sur les politiques à suivre avec les pays en développement, à travers une collaboration efficace et mutuellement avantageuse pour contribuer à la concrétisation des réformes. Conscients qu'il n'existe pas de modèle universel, les ministres ont salué les approches novatrices que sont les examens par pays pluridimensionnels ainsi que les « approches modulaires » appliquées à des pays confrontés à des défis qui présentent les mêmes caractéristiques structurelles. Ils ont aussi invité l'OCDE à réévaluer et, le cas échéant, à adapter ses cadres et mécanismes pour les appliquer à des contextes nationaux plus diversifiés. Les ministres ont souligné la nécessité de hiérarchiser les priorités et de définir un calendrier des activités de suivi en vue d'une mise en œuvre efficace de la Stratégie. 
Les ministres se sont félicités des efforts déployés par

l'OCDE pour mettre au point le Partenariat mondial pour une coopération efficace au service du développement, qui a été avalisé fin 2011 à Busan par un large éventail de pays, au nombre desquels la Fédération de Russie, l'Afrique du Sud, le Brésil, la République populaire de Chine, l'Inde et l'Indonésie, et par plusieurs organisations internationales. Ils ont appelé le Comité d'aide au développement de l'OCDE à continuer d'apporter sa contribution au fonctionnement efficace d'un partenariat soucieux de la diversité qui reconnaît le rôle distinct que peuvent jouer les différents acteurs en faveur d'une croissance inclusive et d'un développement durable, et à consolider le dialogue qu'entretient l'Organisation avec les diverses parties prenantes, notamment les économies émergentes.

Les ministres ont noté avec satisfaction les efforts que déploie actuellement l'OCDE pour rester en première ligne de l'action menée en faveur de l'intégrité et de la lutte contre la corruption et l'ont appelée à renforcer à la fois son rôle de normalisation et ses procédures d'examen mutuel en la matière. Ils ont salué l'arrivée de deux nouveaux membres, la Fédération de Russie et la Colombie, au sein du Groupe de travail sur la corruption dans le cadre de transactions commerciales internationales, et ont accueilli avec satisfaction la récente Recommandation sur les Principes applicables à la gouvernance publique des partenariats publicprivé. Ils ont également réaffirmé leur volonté de mettre en œuvre la Convention anticorruption de l'OCDE et les autres instruments connexes. À cet égard, ils se sont félicités de l'adhésion de la Tunisie, du Maroc et de la Colombie à la Déclaration sur la probité, l'intégrité et la transparence dans la conduite des affaires et de la finance internationales (PIT). Ils ont pris acte de l'Initiative CleanGovBiz, qui s'appuie sur la Déclaration PIT, ainsi que de la possibilité de procéder à des « vérifications de l'intégrité » en s'inspirant du Manuel pour l'intégrité élaboré par l'OCDE. Enfin, ils ont salué le rôle qu'a joué l'Organisation dans la coordination des efforts déployés par le Partenariat de Deauville du G8 pour améliorer la gouvernance.

Les ministres ont souligné l'importance de l'adhésion de la Fédération de Russie à l'OCDE, pour l'Organisation et pour la Fédération de Russie elle-même, et ont insisté sur le fait que cette adhésion devrait être considérée comme un moyen de moderniser l'économie russe et d'améliorer le bien-être de ses citoyens. Ils se sont en outre félicités de l'adhésion de la Fédération de Russie à l'Agence pour l'énergie nucléaire et ont appelé à une coopération constructive qui apportera à l'Agence un soutien dans la mission qui est la sienne de maintenir et d'approfondir, par l'intermédiaire de la coopération internationale, les bases scientifiques, technologiques et juridiques indispensables à une utilisation sûre, respectueuse de l'environnement et économique de l'énergie nucléaire à des fins pacifiques. Ils ont également félicité la Fédération de Russie pour son accession à l'OMC et encouragé les autorités russes à prendre toutes les

Séance d'ouverture de la RCM 2012 : Tous à bord - Des politiques en faveur d'une croissance inclusive et de l'emploi

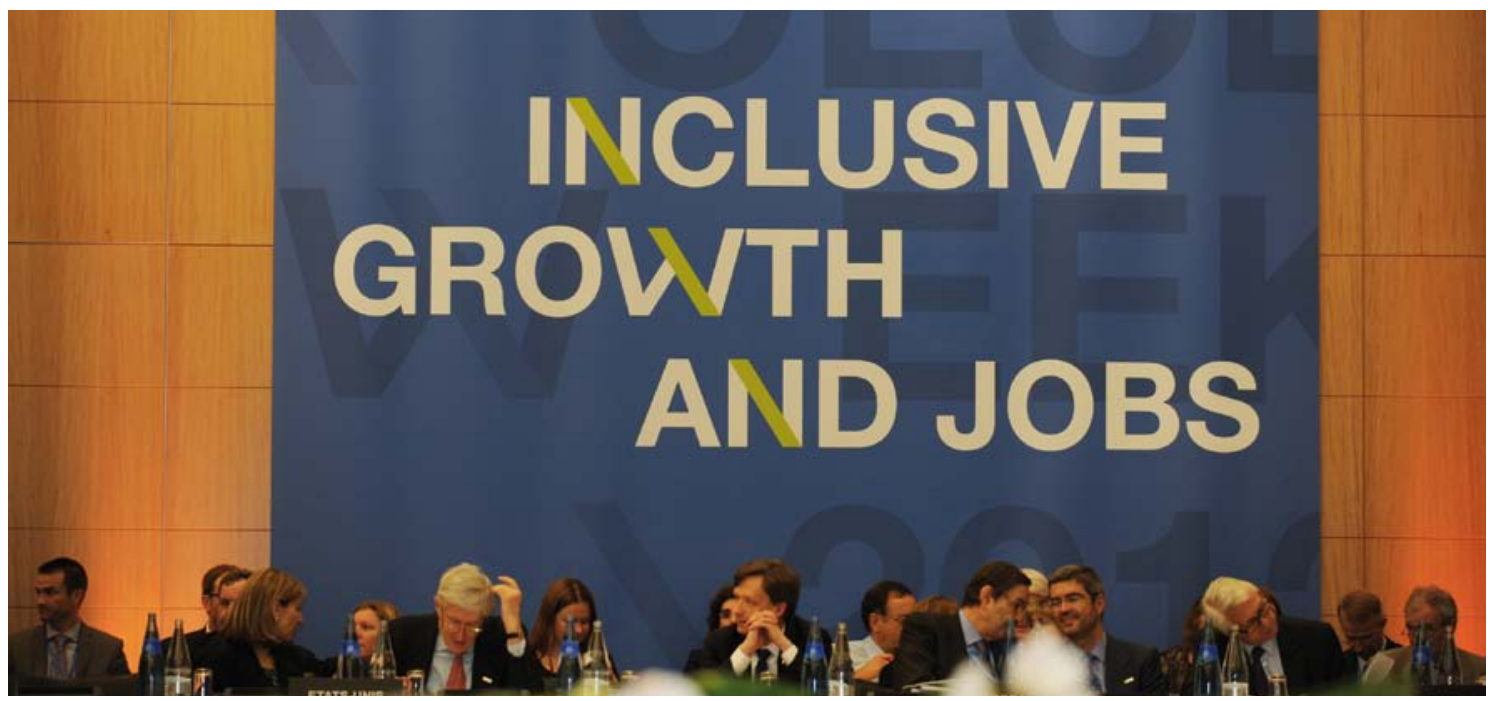


Angel Gurría, Secrétaire général de l'OCDE, et Robert Hormats, Sous-secrétaire

à la Croissance économique, à l'Énergie et à l'Environnement, ministère des Affaires étrangères, États-Unis, au Forum de l'OCDE

dispositions nécessaires pour mener à bonne fin le processus d'adhésion à l'OCDE, conformément à la Feuille de route de 2007. Ils ont salué la volonté de la Fédération de Russie de se conformer aux normes requises pour être membre de l'OCDE. Ils ont appelé les autorités russes à s'engager dans tous les domaines de l'action publique et souligné l'importance de l'application et de la mise en œuvre de l'état de droit en en améliorant l'efficacité. Ils ont rappelé que les normes et les valeurs rigoureuses de l'OCDE doivent être

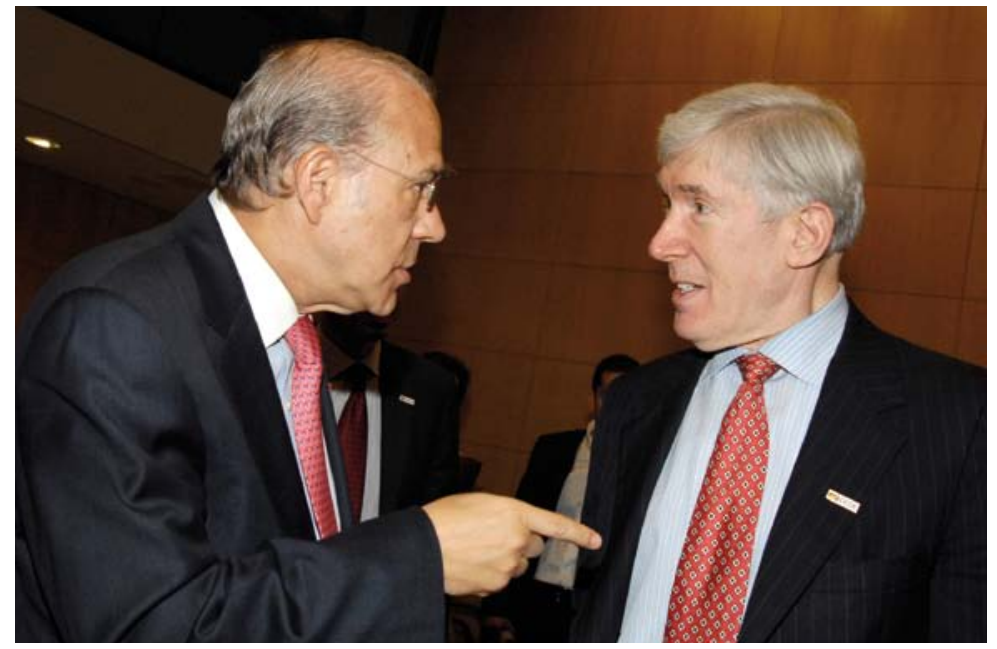

respectées et ont prévenu que la transition vers la mise en application de ces normes et valeurs ne devait pas être exagérément longue.

Les ministres ont salué les efforts déployés par l'OCDE et les progrès accomplis pour renforcer les relations avec les partenaires clés (Afrique du Sud, Brésil, République populaire de Chine, Inde et Indonésie), étape essentielle sur le chemin qui permettra de donner corps à l'appel à faire de l'Organisation un réseau mondial et lancé dans la Vision d'avenir du

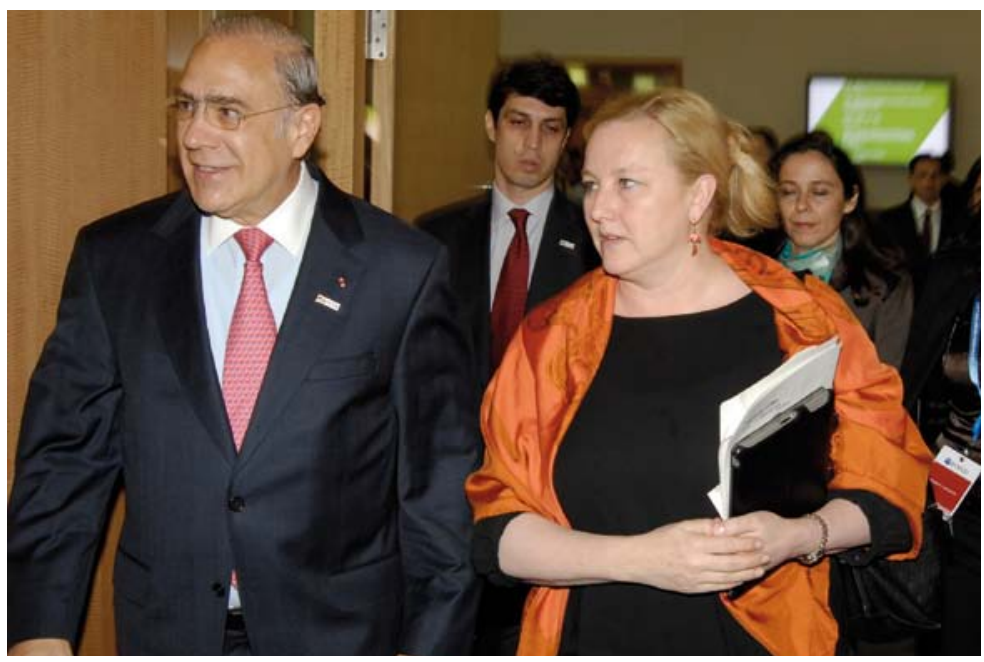

Ewa Björling, ministre du Commerce extérieur de Suède, et Angel Gurría, Secrétaire général de l'OCDE, au Forum de l'OCDE intégrateur pour l'action publique $50^{\circ}$ anniversaire de l'OCDE. Ils ont souligné que cette coopération avec les partenaires clés revêt une importance stratégique et présente un avantage mutuel, et qu'elle est essentielle pour conserver à l'Organisation sa pertinence et son caractère intégrateur. Ils ont insisté sur l'importance d'une action ciblée sur les besoins effectifs des partenaires clés et sur la nécessité d'être à l'écoute de leurs points de vue, tout en restant fidèles aux normes et valeurs de l'Organisation. Ils ont appelé le Secrétaire général à étudier les moyens concrets de faciliter et d'approfondir ces relations, de travailler sur des programmes stratégiques spécifiques présentant un intérêt mutuel en s'appuyant par exemple sur des accords cadre, une participation accrue de ces pays aux travaux des comités, une communication renforcée, et la possible mise en place, dans les capitales, de points de contact spécialisés. Ils ont pris note de la manifestation formelle d'intérêt pour l'adhésion exprimée par certains pays et ont demandé, dans le respect des normes et procédures de l'Organisation, que soit engagée une réflexion générale 


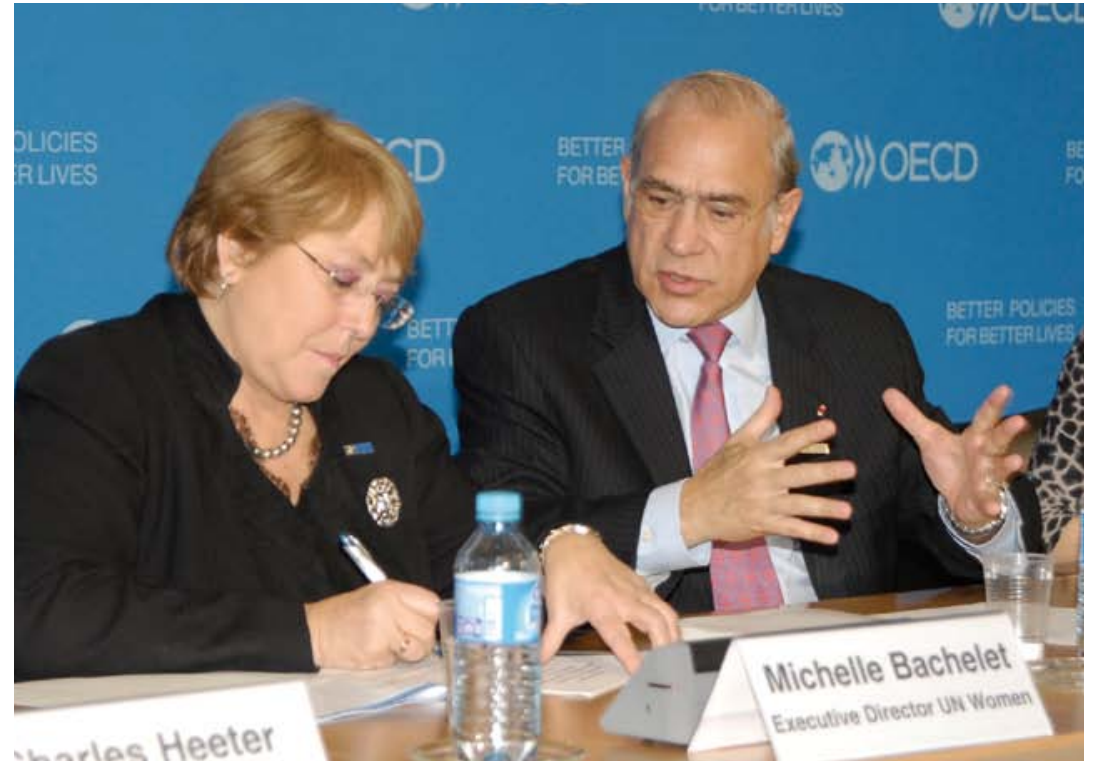

Michelle Bachelet, Directrice générale de l'ONU Femmes, et Angel Gurria, Secrétaire général de l'OCDE, Iors d'une conference de presse au Forum de l'OCDE

au sein du Conseil sur la marche à suivre, y compris une discussion sur les critères d'adhésion.

Les ministres ont fait part de leur intention d'approfondir le dialogue sur les politiques à suivre avec d'autres pays et régions sur la base de l'avantage et de l'intérêt mutuels. Ils sont d'avis que l'évolution vers plus d'intégration suppose également un renforcement des initiatives régionales en Amérique latine, en Eurasie, en Europe du Sud-Est et en Afrique subsaharienne, en plus de l'Asie du Sud-Est qui figure déjà au rang des priorités stratégiques de l'Organisation. Par ailleurs, ils attendent avec intérêt la conclusion rapide des travaux consacrés à l'élaboration de nouvelles règles simplifiées applicables à la participation de pays partenaires aux travaux des organes de l'OCDE.

Les ministres se sont félicités du soutien apporté par l'OCDE aux gouvernements des pays de la région MENA dans leurs efforts de réforme et aux donneurs pour qu'ils redéploient leurs programmes d'aide en fonction des grandes priorités de la région. Ils ont souligné l'importance des réformes économiques qui doivent aller de pair avec les réformes politiques, présenter une forte composante sociale et déboucher sur une expansion des échanges et de l'investissement grâce à l'intégration régionale. Ils ont réaffirmé leur soutien en faveur de relations de travail avec cette région, dont les besoins de développement sont énormes et nécessitent une réaction adaptée. Ils ont en conséquence appelé à une intensification des relations de travail entre l'OCDE et cette région, qui devront aller au-delà des activités d'ouverture habituelles de l'Organisation et viser des résultats concrets. Les ministres ont également fait valoir combien perspective à long terme et d'adopter une approche dictée par la demande, différenciée et individualisée, en même temps qu'une approche il importe de s'inscrire dans une régionale renforcée. Ils se sont félicités du rôle actif joué par l'OCDE au sein du Partenariat de Deauville. Ils ont exprimé leur soutien aux travaux de l'OCDE dans le cadre du projet sur l'administration ouverte et salué sa mise en œuvre en Tunisie et en Jordanie. Les ministres ont en outre encouragé l'OCDE à proposer des évaluations structurelles et des outils de suivi, et à apporter un soutien thématique afin de soumettre des conseils et des solutions propres à améliorer la gouvernance et l'intégrité, l'investissement, la justice sociale, les performances économiques et la création d'emplois, l'entrepreneuriat, l'égalité homme-femme, l'éducation et le développement territorial. Ils ont également salué les efforts déployés par les pays de la région MENA pour adopter des instruments de l'OCDE, en particulier l'adhésion de la Tunisie à la Déclaration de l'OCDE sur l'investissement international et les entreprises multinationales. Ils ont également pris acte de l'apport des pays membres à l'appui du programme MENA de l'OCDE.

Les ministres ont salué les importantes contributions apportées par l'OCDE à l'architecture de la gouvernance internationale, en particulier aux processus engagés dans le cadre du G8 et du G20, dans les domaines où l'Organisation possède un avantage comparatif, notamment en liaison avec le Cadre pour une croissance forte, durable et équilibrée, la sécurité alimentaire, la croissance verte, la fiscalité, la lutte contre la corruption, les échanges, le développement, l'éducation financière, la protection financière des consommateurs, la gestion des risques de catastrophe et l'emploi. Ils se sont également félicités du rôle actif que joue l'OCDE face aux défis mondiaux. 


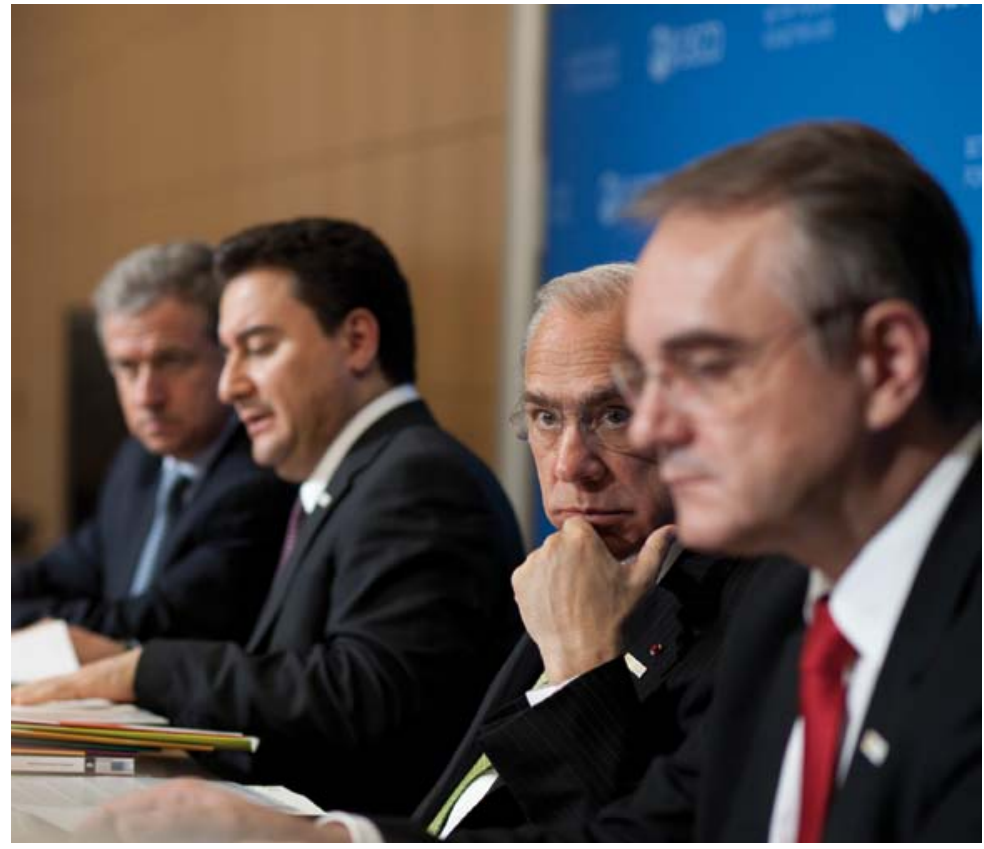

(gauche/droite) Felipe Larraín, ministre des Finances du Chili, Ali Babacan, Vice-Premier ministre délégué aux Affaires économiques et financières de Turquie, Angel Gurría, Secrétaire général de l'OCDE, et Waldemar Pawlak, Vice-Premier ministre de Pologne, à la conférence de presse de clôture de la Réunion du Conseil au niveau des ministres
Enfin, les ministres ont encouragé l'OCDE à redoubler d'efforts pour améliorer son efficacité, son efficience et sa transparence, et ont salué son engagement en faveur des données ouvertes ainsi que les efforts qu'elle déploie pour faire siennes les meilleures pratiques de gestion. Ils ont examiné plusieurs rapports et des propositions concernant de nouveaux domaines de travail et ont noté que, pour être menés à bonne fin, ces projets devraient être pris en compte dans le prochain Programme de travail et Budget de l'Organisation, et adaptés en fonction des ressources financières.

Pour plus d'informations sur la Réunion du Conseil de l'OCDE au niveau des ministres, voir www.oecd.org/fr/rcm

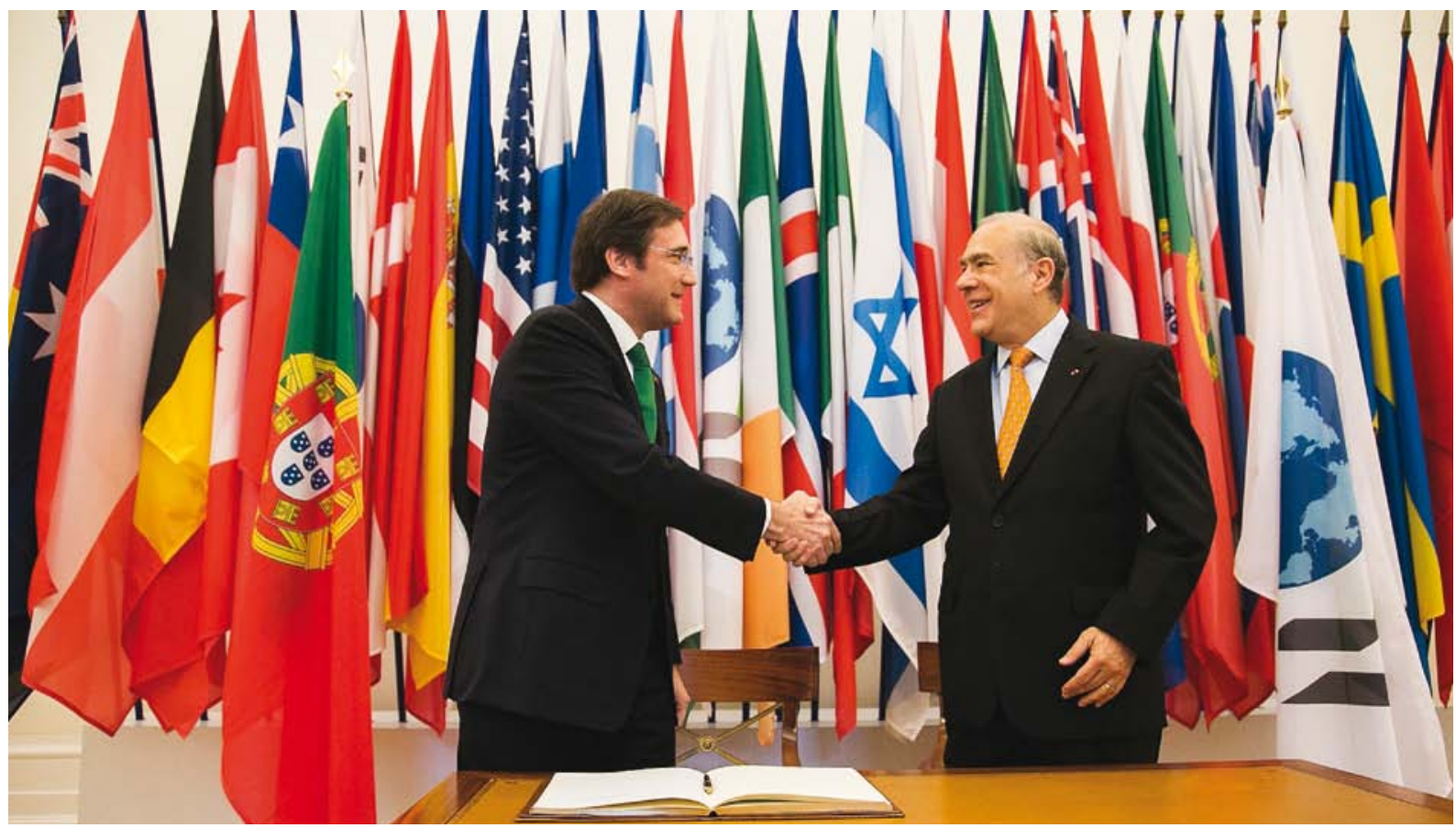

Pedro Passos Coelho, Premier ministre du Portugal, et Angel Gurría, Secrétaire général de l'OCDE, lors de la visite officielle du Premier ministre à l'OCDE, mai 2013 


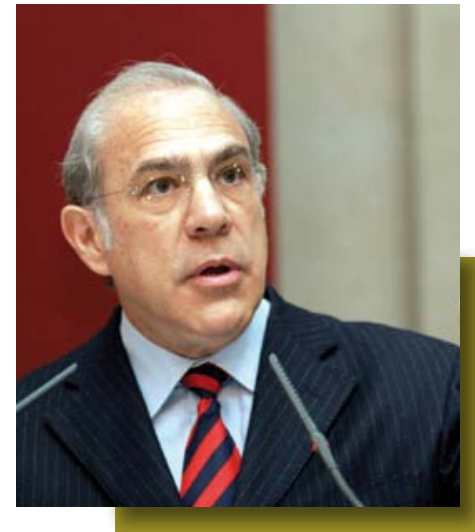

Secrétaire général de l'OCDE depuis juin 2006, Angel Gurría a renforcé la mission de l'OCDE : promouvoir des "politiques meilleures pour une vie meilleure ». Il s'attache à promouvoir une Organisation ouverte à tous et réactive aux besoins des pays membres et partenaires, dans les difficultés du contexte actuel. Il a ouvert la porte à de nouveaux membres et renforcé les liens avec les économies émergentes. Le Secrétaire général Angel Gurría a renforcé le rôle et l'impact de l'OCDE en tant que soutien des bonnes pratiques et des réformes politiques.Il a lancé des initiatives majeures en faveur de hausse de la productivité et de la croissance, concernant notamment l'innovation, la croissance verte, la parité, le développement, les compétences ou encore l'eau. En 2012, il a initié les Nouvelles approches face aux défis économiques, une réflexion engageant l'ensemble de l'Organisation et visant à diffuser les leçons apprises de la crise économique, à actualiser les cadres analytiques de l'OCDE et à développer un programme exhaustif pour une croissance inclusive et durable.

\section{Cabinet du Secrétaire général}

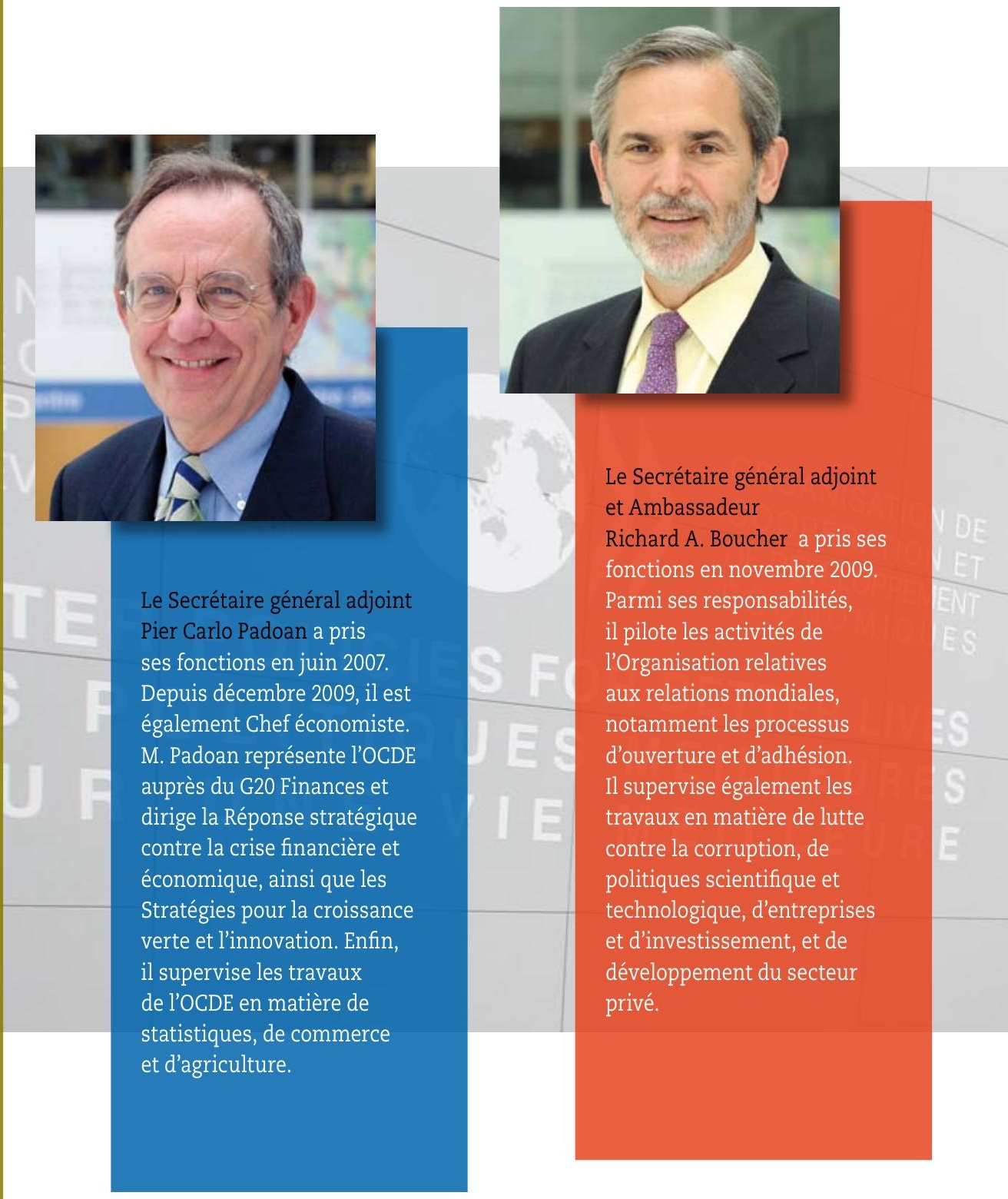




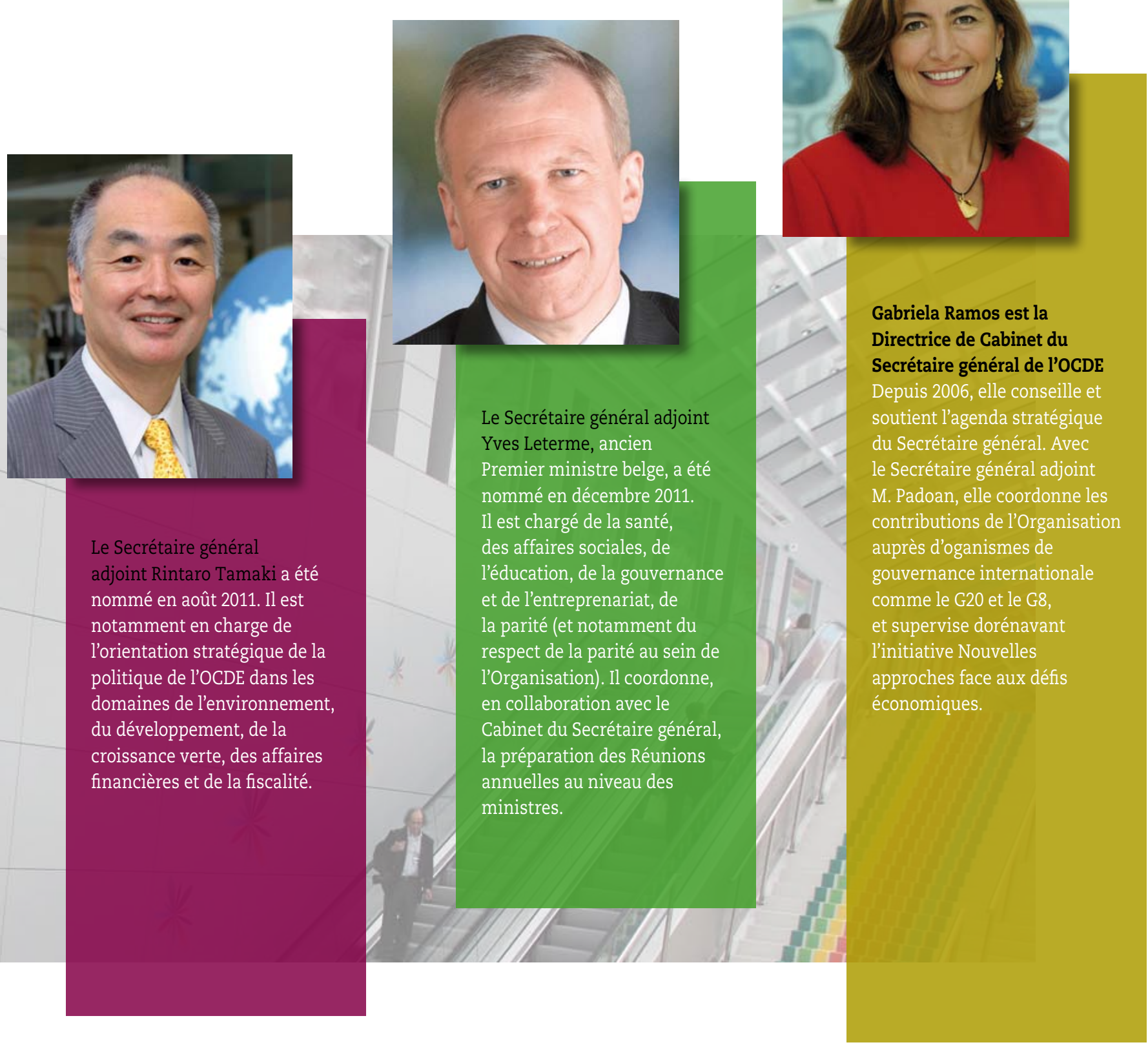




\section{Organigramme}

www.oecd.org/organigramme

\section{SECRÉTARIAT GÉNÉRAL}

SECRÉTAIRE GÉNÉRAL

Angel Gurría

CABINET DU SECRÉTAIRE GÉNÉRAL

Gabriela Ramos, Directrice de cabinet du Secrétaire général de l'OCDE

Sherpa aux G20/G8, NAEC

Secrétariat des relations mondiales Marcos Bonturi, Directeur

Direction des affaires juridiques Nicola Bonucci, Directeur

Direction des relations extérieures et de la communication Anthony Gooch, Directeur

Audit interne et évaluation Peter Stokhof, Directeur adjoint et Auditeur interne principal Kevin Williams, Chef de l'évaluation en profondeur

Secrétariat du Conseil et du Comité exécutif Patrick van Haute, Directeur

\section{ORGANES SPÉCIAUX}

\section{Centre de développement}

Mario Pezzini

Directeur
Agence internationale de l'énergie

Maria van der Hoeven Directrice exécutive

\section{SECRÉTAIRES GÉNÉRAUX} ADJOINTS

Pier Carlo Padoan

Chef économiste - Questions stratégiques

Représentant de l'OCDE auprès du G20 Finances

Richard A. Boucher

Relations mondiales

Rintaro Tamaki

Cohérence des politiques

Yves Leterme

Politique sociale et gouvernance
Agence pour l'énergie nucléaire

Luis Echávarri

Directeur général 


\section{Mai 2013}

\section{DIRECTIONS}

\begin{tabular}{|c|c|c|}
\hline $\begin{array}{l}\text { Département des } \\
\text { affaires économiques }\end{array}$ & $\begin{array}{l}\text { Direction des affaires } \\
\text { financières et des } \\
\text { entreprises }\end{array}$ & $\begin{array}{l}\text { Direction de la } \\
\text { coopération pour le } \\
\text { développement }\end{array}$ \\
\hline $\begin{array}{l}\text { Pier Carlo Padoan } \\
\text { Chef économiste }\end{array}$ & $\begin{array}{l}\text { Carolyn Ervin } \\
\text { Directrice }\end{array}$ & $\begin{array}{l}\text { Jon Lomoy } \\
\text { Directeur }\end{array}$ \\
\hline $\begin{array}{l}\text { Direction de l'emploi, } \\
\text { du travail et des }\end{array}$ & \multirow{2}{*}{$\begin{array}{l}\text { Centre pour } \\
\text { l'entrepreneuriat, } \\
\text { les PME et le } \\
\text { développement local } \\
\text { Sergio Arzeni } \\
\text { Directeur }\end{array}$} & \multirow{2}{*}{$\begin{array}{l}\text { Direction de } \\
\text { l'environnement } \\
\text { Simon Upton } \\
\text { Directeur }\end{array}$} \\
\hline $\begin{array}{l}\text { Stefano Scarpetta } \\
\text { Directeur }\end{array}$ & & \\
\hline $\begin{array}{l}\text { Centre de politique } \\
\text { et d'administration }\end{array}$ & $\begin{array}{l}\text { Direction de la } \\
\text { science, de la } \\
\text { technologie et de } \\
\text { l'industrie }\end{array}$ & $\begin{array}{l}\text { Direction } \\
\text { des statistiques }\end{array}$ \\
\hline $\begin{array}{l}\text { Pascal Saint-Amans } \\
\text { Directeur }\end{array}$ & $\begin{array}{l}\text { l'industrie } \\
\text { Andrew Wyckoff } \\
\text { Directeur }\end{array}$ & $\begin{array}{l}\text { Martine Durand } \\
\text { Directrice }\end{array}$ \\
\hline
\end{tabular}

Direction

des échanges et

de l'agriculture

Ken Ash

Directeur
Direction de

l'éducation et des compétences

Barbara Ischinger Directrice

\section{Direction}

exécutive

Anthony Rottier

Directeur
Direction

de la gouvernance publique et du développement territorial

Rolf Alter

Directeur

\section{ENTITÉS SPÉCIALES}

Club du Sahel et de l'Afrique de l'Ouest

Laurent Bossard

Directeur

\section{Forum international des transports}

José Viegas

Directeur
Forum pour le partenariat avec l'Afrique

David Batt Directeur
Groupe d'action financière

Rick McDonell Secrétaire Éxécutif 



\section{A C TIVITÉ S PRINCIPALES}

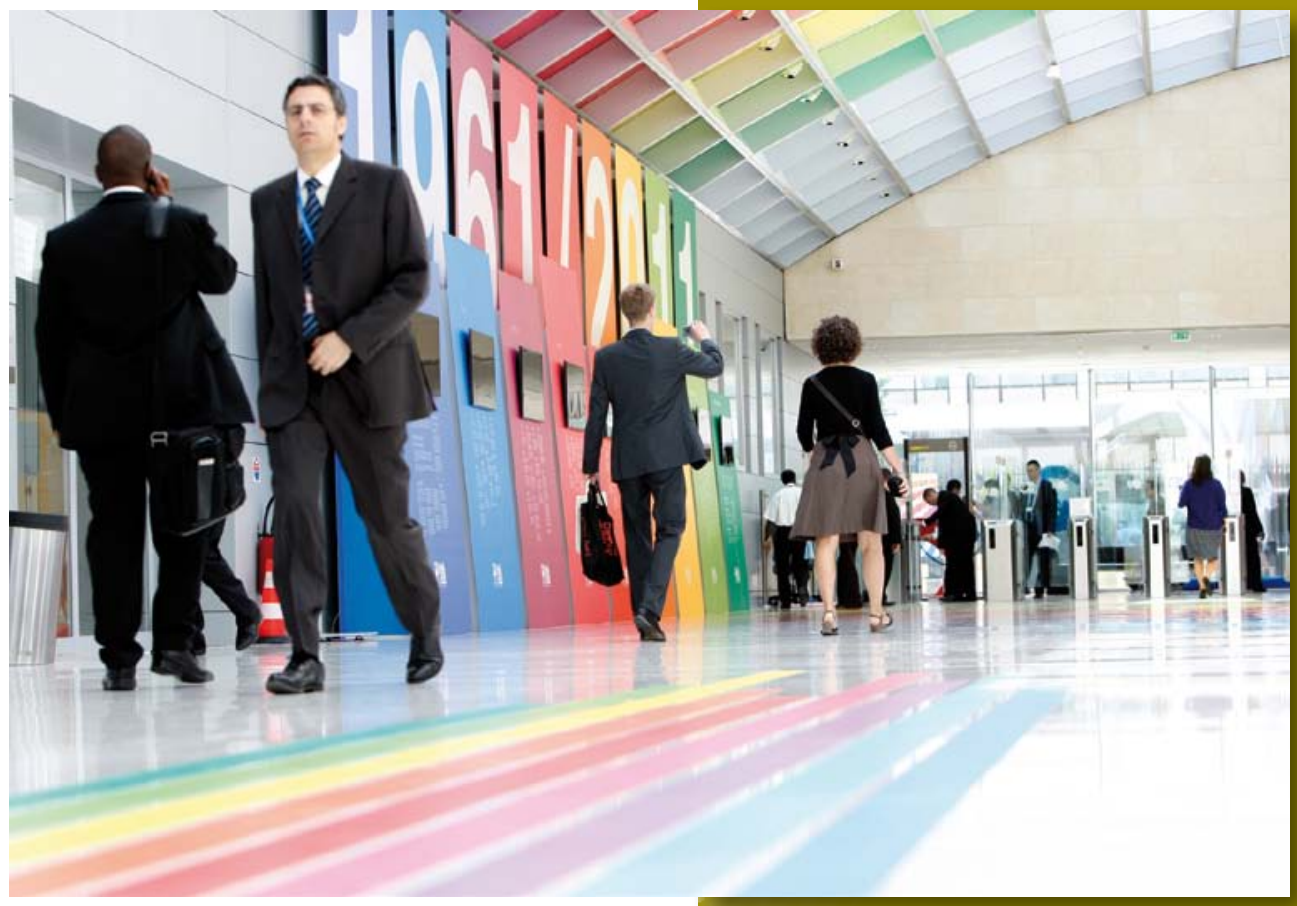




\section{Nouvelles approches face aux défis économiques (NAEC)}

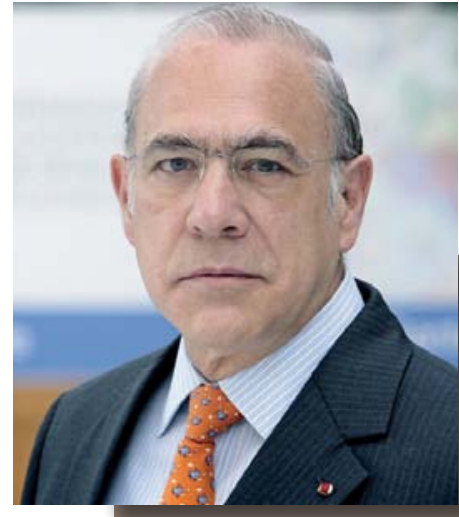

Angel Gurría Secrétaire général de l'OCDE Courriel : naec@oecd.org

« La crise mondiale a représenté un coup de semonce pour les décideurs du monde entier. Les défaillances des marchés et de la gouvernance ont conduit à la pire crise financière, économique et de l'emploi de notre époque, et nous en affrontons encore les retombées désastreuses. Dans le même temps, nous sommes confrontés aux conséquences des grandes tendances actuelles, telles que la mondialisation et le basculement de la richesse, la croissance démographique et le vieillissement de la population, les défis environnementaux et la raréfaction des ressources naturelles, ou encore la demande croissante de compétences résultant du progrès technologique. Dans ce contexte, un simple retour au statu quo n'est pas envisageable. $\gg$
L'initiative Nouvelles approches face aux défis économiques (NAEC) a été lancée lors de la réunion du Conseil de l'OCDE au niveau des ministres de 2012. Elle a été conçue comme un processus de réflexion à l'échelle de l'ensemble de l'Organisation, pour tirer les leçons de la crise afin d'enclencher un processus d'amélioration continue des grilles d'analyses utilisées par l'OCDE et des conseils qu'elle dispense.

L'objectif ultime de l'initiative NAEC est d'établir un programme d'action stratégique pour instaurer une croissance inclusive et durable. À cette fin, cette initiative permettra dans un premier temps de réfléchir aux enseignements de la crise, de recenser les domaines où il est nécessaire d'adapter les grilles d'analyse de l'OCDE et d'évaluer le potentiel des nouveaux outils et des nouvelles approches économiques. Dans un deuxième temps, il s'agira de prendre acte du rôle de la croissance économique en tant que moyen, et non en tant que fin en soi de l'action publique, en mettant l'accent sur le bien-être et toutes les améliorations spécifiques qui influencent la qualité de vie des populations (santé, emploi ou services de proximité, par exemple). Troisièmement, l'initiative NAEC vise à mieux comprendre le caractère complexe et interdépendant de l'économie mondiale, et à trouver de meilleures manières d'aborder les arbitrages et synergies entre différents domaines de l'action publique, par exemple entre croissance, inégalités, stabilité et environnement. Enfin, cette initiative aspire à aider les pouvoirs publics à cibler, hiérarchiser et conjuguer les réformes à l'appui d'une croissance durable et inclusive, ce qui suppose d'améliorer les capacités réglementaires et institutionnelles et de rétablir la confiance accordée aux gouvernements.

Un groupe de travail a été constitué pour coordonner et conduire ce projet. Ce Groupe NAEC comprend des représentants des pays membres de l'OCDE et des pays partenaires, des comités spécialisés de l'Organisation, ainsi que du Comité consultatif économique et industriel (BIAC) et de la Commission syndicale consultative (TUAC). Il est présidé par le Secrétaire général de l'OCDE. Pour faire progresser cette initiative, le Secrétaire général a nommé un coordonnateur NAEC qui travaillera sous la supervision du Directeur de Cabinet du Secrétaire général et Sherpa de l'OCDE au G20.

\section{Publications principales}

- Nouvelles approches face aux défis économiques - Document cadre

- Nouvelles approches face aux défis économiques - Mandat

- Note de synthèse pour la première réunion du Groupe NAEC le 24 octobre 2012

- Rapport d'étape aux ministres

\section{Manifestations principales}

- Première réunion du Groupe NAEC, Paris, 24 octobre 2012

- Deuxième réunion du Groupe NAEC, Paris, 11 février 2013 


\section{Structure d'organisation des travaux du groupe NAEC}

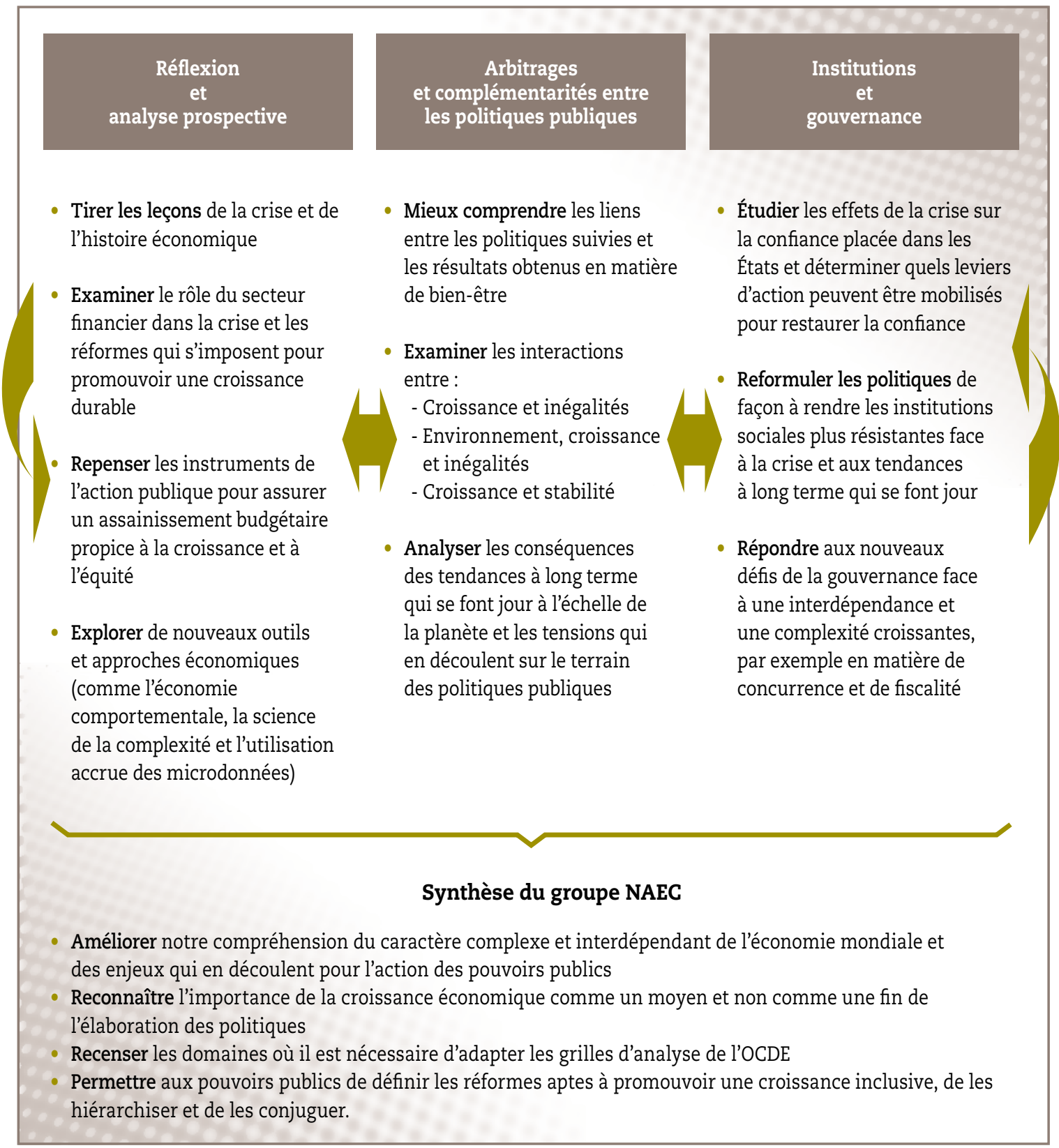




\section{Cabinet du Secrétaire général}

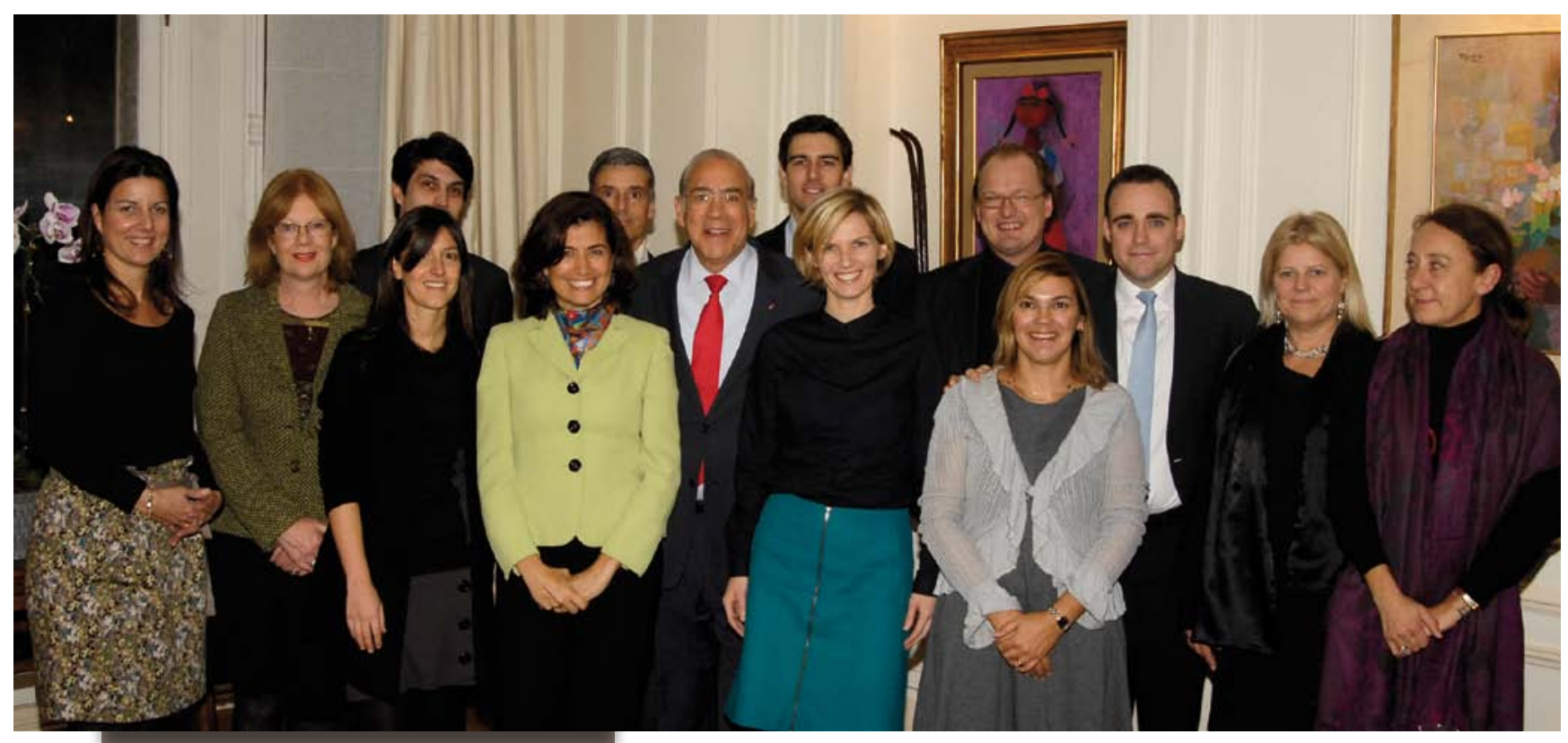

Cabinet du Secrétaire général Courriel : secretary.general@oecd.org

« L'impact, la pertinence et l'inclusivité sont notre raison d'être. Ce sont des mots d'ordre d'autant plus importants dans le contexte de la crise actuelle, où la richesse et la diversité des travaux menés par l'OCDE peuvent appuyer l'effort de réforme des pays membres et des pays partenaires en les aidant à "penser structurel", "penser social" et "penser vert". »)
Le Cabinet du Secrétaire général (OSG) aide le Secrétaire général à donner corps à sa vision stratégique pour l'Organisation. Sous la conduite du Directeur de Cabinet et Sherpa au G20, et du Directeur adjoint, le Cabinet du Secrétaire général s'emploie à accroître la pertinence, l'impact et la visibilité des activités de l'OCDE, pour veiller à ce que l'Organisation reste à la pointe de l'analyse stratégique, pour prodiguer en temps utile des conseils ciblés, et pour assurer, avec le concours des Secrétaires généraux adjoints, la supervision des travaux de l'Organisation et la création de synergies.

Pour être aux avant-postes des évolutions des politiques publiques et servir aux mieux les décideurs des pays membres et des pays partenaires, le Cabinet du Secrétaire général a lancé et supervise l'initiative relative aux Nouvelles approches face aux défis économiques et les travaux sur la Croissance inclusive. Le Cabinet et les Secrétaires généraux adjoints participent en outre à la promotion et à la supervision de projets horizontaux consacrés aux compétences, à l'innovation, à la croissance verte et à la parité.

Durant l'année 2012, l'agenda du Cabinet a une nouvelle fois été extrêmement chargé, avec plus d'un millier de réunions au siège de l'OCDE et plus de 49 visites du Secrétaire général à l'étranger. Le Cabinet a également rédigé plus de 220 allocutions, traité en moyenne 500 courriels par jour et élaboré plus de 20000 pages de notes d'information. Le principal objet des travaux a consisté à conseiller les pays membres et à les épauler dans leur lutte contre la crise économique et ses conséquences sociales. Les Secrétaires généraux adjoints ont également assumé leur part de cet emploi du temps très intense et contribué à la supervision d'axes de travail et de produits spécifiques dans divers domaines d'intervention afin de donner le maximum d'écho aux activités de l'OCDE. 
Avec le concours du Cabinet du Secrétaire général, l'Organisation a réalisé d'importantes avancées en 2012 pour renforcer son impact sur les agendas nationaux. Le Cabinet a été en particulier au centre des efforts déployés pour produire les brochures de la série Politiques meilleures et pour organiser nombre de visites et manifestations au plus haut niveau de représentation, notamment :

- Le Séminaire sur la croissance, en présence du Président du Conseil italien, M. Monti;

- Les discussions sur la cohésion sociale, avec la Présidente élue de la Corée, Mme Park Geun-hye;

- La visite du Président élu du Mexique, M. Peña Nieto, et la présentation de la publication Getting it Right, qui a largement inspiré le programme du nouveau gouvernement mexicain, et la signature d'un accord de partenariat;

- Des discussions de fond sur les réformes des politiques publiques avec le Président de la République française, M. François Hollande, la Chancelière allemande, Mme Merkel et des responsables d'organisations internationales;

- Un dialogue sur les politiques à suivre et des séminaires avec les Premiers ministres de la Slovaquie et du Portugal, le premier Vice-Premier ministre de la Fédération de Russie, ainsi qu'un examen à mi-parcours du programme du gouvernement finlandais, avec le Premier ministre de la Finlande et son Cabinet.

- Enfin, un dialogue et des discussions au sein du Conseil avec les Présidents de l'Islande, d'Israël, de la Colombie et du Pérou.

Par ailleurs, le Cabinet du Secrétaire général coordonne, en étroite collaboration avec le Secrétaire général adjoint Yves Leterme, la préparation des réunions ministérielles annuelles, tant sur le fond que sur le plan de l'organisation. En outre, le Cabinet organise des échanges avec des penseurs éminents tels que Daniel Kahneman, James Kenneth Galbraith ou Alan Kirman, afin de veiller à ce que l'Organisation soit toujours en phase avec les grandes évolutions du monde universitaire. Pour l'avenir, le Cabinet s'appliquera à renforcer le rôle de plateforme pour les pays membres et les pays partenaires que joue l'OCDE. Premièrement, en s'appuyant sur la série Politiques meilleures afin de garantir l'actualité et la meilleure adéquation de nos conseils à la spécificité des besoins des différents pays. Deuxièmement, en recherchant les moyens de renforcer son soutien aux représentants des autorités nationales pour mettre en œuvre les réformes, par le biais de programmes, d'ateliers et de formations pour le renforcement des capacités. Troisièmement, en mettant à profit notre expérience de l'organisation de visites au plus haut niveau en institutionnalisant un programme ouvert à des personnalités de premier plan et à leur vision du monde.

\section{Principales publications}

Série politiques meilleures :

- Mexique : Perspectivas OCDE: México Reformas para el Cambio, janvier 2012

- Japon : Policies for a revitalisation of Japan, avril 2012

- France : Promouvoir la Croissance et la Cohésion Sociale, juin 2012

- Mexique : Mejores Políticas para un Desarrollo Incluyente, juillet 2012

- Italie : Reviving Growth and Productivity, septembre 2012

- France: Redresser la compétitivité, octobre 2012 (version actualisée en mars 2013)

- Allemagne : Addressing the Competitiveness Challenges in Germany and the Euro Area, octobre 2012

- Inde : Sustaining High and Inclusive Growth, octobre 2012

- Finlande : Fit for the Future, février 2013

- Slovaquie : Fostering Inclusive Growth and a Job-Rich Recovery, avril 2013

- Fédération de Russie : Modernising the Economy, avril 2013

- Portugal : Reforming the State to Promote Growth, mai 2013 


\section{Bureau du Sherpa}

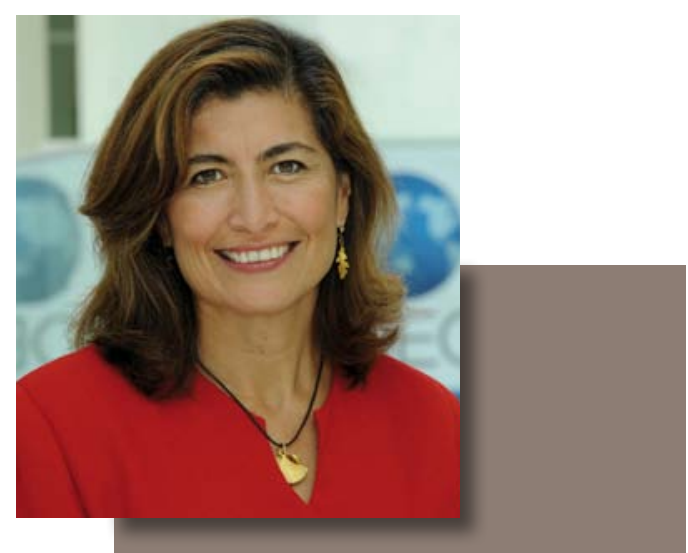

Gabriela Ramos

Directrice de Cabinet du Secrétaire général et Sherpa au G20 Courriel : osg@oecd.org

\section{« Le G20, premier forum}

de coopération économique

internationale, joue un rôle

central dans la promotion

des réformes économiques

internationales indispensables

pour surmonter la crise et

instaurer une croissance forte,

inclusive et durable. Pour

étayer ce processus par des

contributions de fond, des

éléments concrets et des objectifs,

le G20 s'appuie l'expérience,

le savoir-faire et les données

des organisations internationales.

Forte de plus de 50 années

d'expérience, l'OCDE est très

honorée d'apporter sa pierre à cet

édifice depuis 2008, en proposant

des politiques meilleures pour

une vie meilleure. $\gg$
Le Bureau du Sherpa apporte son soutien au Secrétaire général, à son Directeur de Cabinet et Sherpa, ainsi qu'au Chef économiste de l'OCDE, en coordonnant l'ensemble des contributions de l'OCDE aux travaux du G20 et son appui aux présidences successives du G20 et du G8.

En 2012, le gouvernement mexicain a sollicité la contribution et l'appui de l'OCDE aux priorités du G20. Il s'agissait notamment d'intégrer la croissance verte dans les programmes de réformes structurelles des pays du G20 et d'élaborer une "boîte à outils " non contraignante pour une croissance verte inclusive à l'intention des décideurs des économies en développement. Dans le contexte du "Cadre pour une croissance forte, durable et équilibrée ", l'OCDE a pleinement contribué au Plan d'action de Los Cabos en aidant les pays à hiérarchiser les priorités de leurs programmes de réformes structurelles.

À Los Cabos, les dirigeants du G20 se sont également engagés à travailler plus activement avec le Groupe de travail de l'OCDE sur la corruption, et ont adopté un ensemble de principes relatifs aux déclarations de patrimoine, rédigés conjointement par l'OCDE et la Banque mondiale. En octobre 2012, les Sherpas au G20 ont adopté un nouveau Plan d'action anti-corruption, élaboré avec le concours de l'OCDE. L'Organisation a également apporté une contribution majeure aux débats du G20 sur l'emploi, le développement et les échanges, ainsi qu'à la première réunion des ministres du Commerce du G20, à Puerto Vallarta, qui s'est penchée sur les chaînes de valeur mondiales. Il a été demandé à l'OCDE d'approfondir ses travaux sur l'inclusion financière et sur la gestion des risques de catastrophes. L'Organisation a également contribué à élaborer un programme d'action visant à accroître la productivité agricole.

Pour l'avenir, l'OCDE appuie également la Présidence russe du G20 afin que le Sommet de Saint-Pétersbourg puisse donner lieu à des résultats concrets, en particulier un Plan d'action. La Présidence russe s'attache notamment à favoriser le financement de l'investissement. Dans ce but, l'OCDE élabore actuellement un ensemble de Principes de haut niveau. L'Organisation contribue aussi à assurer la viabilité des finances publiques et la justice fiscale, en travaillant sur l'érosion des bases d'imposition et le transfert de bénéfices, ainsi que sur l'échange automatique de renseignements à des fins fiscales. Elle poursuit activement ses travaux sur le développement, l'emploi, la lutte contre la corruption et l'énergie. Également intéressée par les travaux de l'OCDE sur les échanges en valeur ajoutée et les chaînes de valeur mondiales, la Présidence russe accueillera un séminaire en marge de notre Réunion au niveau des ministres du 29 mai 2013.

Le G8 s'appuie également sur l'expertise et le savoir-faire de l'OCDE. Dans le cadre du Partenariat de Deauville, nous soutenons les efforts des pays en transition pour promouvoir la croissance, l'emploi, l'investissement et la bonne gouvernance. En tant qu'institution d'appui à la mise en œuvre, l'OCDE participe au Fonds de transition du Partenariat de Deauville. Lors de la présidence britannique du G8 en 2013, nous avons contribué aux domaines prioritaires que sont la fiscalité, le commerce et la transparence. Le Secrétaire général a été invité au Sommet du G8 en juin pour débattre de ces questions. 


\section{Publications principales}

- Pursuing Strong, Sustainable and Balanced Growth: A Note on Implementation of G20 Structural Reform Commitments, juin 2012

- Boosting Jobs and Living Standards in G20 countries, rapport conjoint du BIT, de l'OCDE, du FMI et de la Banque mondiale, juin 2012

- Action Plan on Effective Practices to Support the Implementation of the High-Level Principles on National Strategies for Financial Education, juin 2012

- Principes de haut niveau sur des stratégies nationales d'éducation financière du Réseau international sur l'éducation financière (INFE), août 2012

- Interim Progress Report on the Action Plan's implementation to support the development of Local Currency Bond Markets, établi par la Banque mondiale, les banques régionales de développement, le FMI, l'OCDE, la BRI et le CSF, juin 2012

- Tax Transparency: Report on Progress on the Global Forum Activities, rapport régulièrement préparé et mis à jour pour les Sommets, juin 2012

- Sustainable Agricultural Productivity Growth and Bridging the Gap for Small Family Farms, rapport coordonné par la FAO et l'OCDE, établi conjointement par Bioversity International, le Consortium du CGIAR, la FAO, le FIDA, l'IFPRI, l'IICA, l'OCDE, la CNUCED, l'Équipe spéciale de haut niveau des Nations unies sur la crise mondiale de la sécurité alimentaire, le PAM, la Banque mondiale et l'OMC, établi pour la réunion des délégués pour l'agriculture tenue à Mexico, mai 2012

- A Toolkit of Policy Options to Support Inclusive Green Growth, document soumis au Groupe de travail du G20 sur le développement par la BAfD, l'OCDE, les Nations unies et la Banque mondiale, juin 2012

- Incorporating Green Growth and Sustainable Development Policies into Structural Reform Agendas, rapport établi pour le G20 par l'OCDE, la Banque mondiale et les Nations unies, juin 2012

- Improving the Assessment of Disaster Risks to Strengthen Financial Resilience, publication spéciale conjointe du G20 établie par les autorités mexicaines et la Banque mondiale (avec le concours de l'OCDE), juin 2012

- OECD Voluntary Framework to Facilitate implementation of Disaster Risk Management, novembre 2012

- Pension Fund Financing for Green Infrastructure and Initiatives, note d'orientation G20/OCDE, juin 2012

- G20 Trade and Investment Measures, rapport conjoint de l'OMC, la CNUCED et l'OCDE, mi-octobre 2011 à mi-mai 2012

- G20 Principles on Asset Disclosure of Public Officials, juin 2012

- Lutter contre l'érosion de la base d'imposition et le transfert de bénéfices, février 2013

\section{Manifestations principales}

- Séminaire de synthèse OCDE-G20 sur les chaines de valeur mondiales, Paris, mai 2013

- Conférence de haut niveau G20-OCDE : "Ensemble contre la corruption : entreprises et pouvoirs publics du G20 ", Paris, avril 2013

- Atelier sur la régulation de l'énergie et le développement durable, Paris, novembre 2012

- Séminaire à haut niveau sur la croissance verte ouvert aux membres de l'OCDE et aux pays partenaires, Paris, mai 2012

- Réunion à haut niveau public-privé sur la lutte contre la corruption, Paris, octobre 2012

- Réunions du Groupe de travail sur la lutte contre la corruption, Paris, octobre 2012

- Groupe de travail Transparence et anti-corruption du B20, Paris, octobre 2012

- Première réunion des ministres du Commerce du G20, Puerto Vallarta, avril 2012

- Sommet du G20 sur le commerce et la promotion des investissements, Mexico, novembre 2012.

- Conférence du Partenariat de Deauville du G8 sur le thème : «Bâtir une administration ouverte, équitable et transparente à l'appui de la croissance et du développement », 24 novembre 2012 


\title{
«Penser horizontal » - Initiatives et projets de I'OCDE pour mieux relever les défis politiques interconnectés
}

\author{
Des défis politiques complexes requièrent des réponses pluridisciplinaires
}

Comme souligné dans les Orientations stratégiques, notre monde de plus en plus complexe nous amène à nous intéresser davantage aux questions transversales ainsi qu'aux synergies à exploiter et aux arbitrages à opérer entre les différents domaines de l'action publique.

L'OCDE est la seule organisation internationale qui réunit en son sein une expertise des politiques économiques, sociales, environnementales, sociales et de l'emploi. Cette « perspective horizontale " est un important pilier de la stratégie du Secrétaire général, qui vise à offrir à ses membres et à ses partenaires toute la valeur ajoutée associée au savoir-faire de l'OCDE, et à refléter les nouvelles réalités et les besoins de nos concitoyens dans un monde caractérisé par la mondialisation et l'interdépendance.

Sur le plan stratégique, cette démarche est incarnée par les Nouvelles approches face aux défis économiques (NAEC), mais aussi par une pluralité d'initiatives et de projets horizontaux tels que la Stratégie pour l'innovation, la Stratégie pour une croissance verte, la Stratégie pour le développement et la Stratégie sur les compétences, ou encore le Projet sur la parité et l'Initiative du vivre mieux.

\section{La Stratégie pour une croissance verte}

La crise économique a coïncidé avec une montée des préoccupations au sujet des pressions exercées sur l'environnement, notamment le changement climatique, qui rend inévitable une transformation des systèmes énergétiques. Il est apparu clairement que le nécessaire "verdissement " de l'activité économique pouvait être une source de croissance en stimulant l'innovation et le développement de nouvelles technologies. La Stratégie de l'OCDE pour une croissance verte insiste d'ailleurs sur le fait que " croissance » et « souci de l'environnement » doivent aller de pair.

En l'occurrence, la Stratégie de l'OCDE vise à favoriser la croissance économique et le développement, tout en veillant à ce que les actifs naturels continuent de fournir les ressources et les services environnementaux sur lesquels repose notre bien-être. À cette fin, les gouvernements doivent catalyser l'investissement et l'innovation qui étaieront une croissance durable et créeront de nouvelles opportunités économiques. Cette démarche horizontale doit s'appuyer sur de nouvelles sources de croissance, mais aussi sur un « verdissement » des pratiques actuelles, en particulier dans le cadre des efforts de sortie de crise.

La Stratégie pour une croissance verte énonce des recommandations pour aider les gouvernements à déterminer les moyens les plus efficaces d'opérer la transition vers une trajectoire de croissance plus verte. Elle préconise notamment d'éliminer les subventions dommageables à l'environnement, de recentrer la fiscalité sur les externalités environnementales et de veiller à ce que les actifs environnementaux soient correctement évalués. Une bonne exécution des politiques dans cette optique peut promouvoir une utilisation plus rationnelle des ressources, ainsi que l'innovation technologique.

L'OCDE a beaucoup progressé dans l'intégration de la croissance verte dans les études économiques et environnementales qu'elle réalise régulièrement, et elle a élargi le champ de ses travaux d'analyse aux défis et aux opportunités propres aux pays en développement. De réels efforts sont faits pour que les principales directions travaillent de concert afin de s'assurer qu'un message cohérent en faveur de la croissance verte émane des recommandations d'action concernant la politique économique, l'innovation, l'investissement, la gouvernance et les politiques sectorielles. Les principaux domaines de travail pour l'année à venir sont notamment les suivants :

- Investissement vert, investisseurs institutionnels et infrastructures

- Lien entre politiques environnementales et résultats économiques

- Poursuite du développement des indicateurs de croissance verte

- Aspects particuliers de la fiscalité verte

- Croissance verte et agriculture

La Stratégie pour une croissance verte est coordonnée par l'Unité de la croissance verte et supervisée par les Secrétaires généraux adjoints Pier Carlo Padoan et Rintaro Tamaki.

Pour plus d'informations, voir : www.oecd.org/fr/croissanceverte/versunecroissanceverte.htm 


\section{La Stratégie pour l'innovation}

Les investissements dans de nouvelles sources de croissance et le renforcement des performances de l'innovation demeurent essentiels pour sortir de la crise. Ils ouvrent la voie à une croissance et une compétitivité internationale plus solides, et peuvent aider à relever toute une série de défis sociaux et environnementaux. Toutefois, dans l'actuel contexte de restrictions budgétaires, l'architecture des politiques de l'innovation est cruciale. Il faut continuer d'investir dans des travaux de recherche, des compétences, des infrastructures et des politiques qui contribuent à renforcer les capacités d'innovation, mais ces politiques doivent être conçues avec soin et atteindre leurs objectifs en toute efficience. Parallèlement, il est plus important que jamais d'accomplir les réformes structurelles - des marchés de produits, du travail et des capitaux - susceptibles de libérer l'innovation et l'entrepreneuriat.

Après le lancement de la Stratégie de l'OCDE pour l'innovation en 2010, l'innovation s'est pleinement intégrée à la palette de stratégies et de projets horizontaux de l'Organisation. La croissance verte est très dépendante de politiques et d'incitations précises visant à déverrouiller l'innovation verte; et le développement impose de mettre sans équivoque l'accent sur l'innovation, en veillant toutefois à l'adapter aux besoins particuliers des économies en développement. Des Examens des politiques d'innovation concernant des pays tant membres (Mexique ou Suède, par exemple) que non membres (Asie du Sud-Est, Fédération de Russie) de l'OCDE ont par ailleurs cherché à ajuster les recommandations de la Stratégie pour l'innovation aux contextes nationaux. Et le travail visant à approfondir la compréhension de l'innovation se poursuit : ainsi, de récentes études montrent que dans certains pays de l'OCDE, les investissements dans les actifs intellectuels (c'est-à-dire notamment les logiciels, les dessins et modèles, les données, la R-D et le capital organisationnel) sont désormais plus importants que ceux consacrés aux actifs corporels, ce qui impose un ajustement de l'action publique dans bien des domaines.

Prochaines étapes et perspectives : nous poursuivrons les efforts visant à mieux mesurer et évaluer les investissements consacrés à l'innovation - y compris les crédits d'impôt pour la R-D, qui constituent dans maints pays de l'OCDE l'outil de soutien de l'innovation le plus onéreux. D'autres examens nationaux des politiques d'innovation seront également menés, par exemple pour la France, la Colombie, le Vietnam et les Pays-Bas. Les recherches sur les politiques relatives au capital intellectuel, sans omettre le domaine des données massives (big data) et des droits de propriété intellectuelle, seront approfondies. Et dans le contexte du projet NAEC de l'OCDE, des analyses seront menées sur les perspectives en matière d'innovation, l'objectif étant de mieux appréhender les déterminants de la croissance de la productivité à long terme. Enfin, la Stratégie pour l'innovation elle-même sera revisitée afin d'actualiser et d'affiner ses recommandations et analyses sur la base des travaux conduits depuis 2010, y compris en veillant à ce que l'innovation nourrisse la croissance inclusive.

Les travaux concernant l'innovation sont coordonnés par la Direction de la science, de la technologie et de l'industrie, et supervisés par les Secrétaires généraux adjoints Pier Carlo Padaon et Richard Boucher.

Pour plus d'informations, voir www.oecd.org/fr/sites/strategiedelocdepourlinnovation 


\section{La Stratégie sur les compétences}

Les compétences sont la monnaie du xxI siècle - elles joueront un rôle décisif pour les économies, les sociétés et les perspectives d'avenir des individus. La Stratégie de l'OCDE sur les compétences a été adoptée par les ministres lors de la réunion 2012 du Conseil au niveau des ministres (RCM). Elle vise à renforcer les systèmes de compétences en favorisant la cohérence du développement, l'activation et l'utilisation effective des compétences pour promouvoir la prospérité économique et la cohésion sociale, reflétant en cela l'importance donnée à la notion d' « employabilité tout au long de la vie ».

Les dépenses dans l'éducation et dans les compétences sont un investissement pour l'avenir, et développer les compétences des jeunes et des adultes doit être une priorité, particulièrement dans un monde où les actifs intellectuels sont déterminants et où les jeunes ont beaucoup de difficultés à s'insérer dans le monde du travail lorsqu'ils n'ont pas les compétences adéquates. Mais cela ne suffit pas : il faut aussi modifier les politiques et les pratiques, sur le marché du travail et dans le domaine social, qui découragent les individus d'apporter leurs compétences sur le marché du travail ou en empêchent l'utilisation efficace. La Stratégie de l'OCDE sur les compétences souligne l'importance qu'il y a à adopter une approche pangouvernementale et transversale en matière de compétences et à engager toutes les parties prenantes, notamment les employeurs et les syndicats, pour obtenir de meilleurs résultats économiques et sociaux.

L'OCDE travaille actuellement avec différents pays, pris individuellement, avec lesquels elle s'emploie à élaborer des stratégies plus efficaces en matière de compétences, au niveau national et local, en appliquant une approche collaborative innovante. Il s'agit d'articuler la coopération des différentes parties prenantes autour des besoins et des priorités du pays, en appliquant les instruments et les techniques de l'OCDE de nature à favoriser une approche horizontale dans les pays, afin d'identifier les atouts du système national en matière de compétences et les défis auxquels il est confronté, d'identifier les options qui se présentent aux pouvoirs publics et de concevoir un plan d'action pour mettre en œuvre de meilleures politiques qui reflètent une appropriation du projet à l'échelon national et l'engagement de toutes les parties prenantes. Ce type de coopération est en cours, actuellement, avec l'Autriche, la Corée et la Norvège, et des projets sont à l'étude avec plusieurs autres pays. Dans le cadre de ce processus, l'OCDE travaille aussi plus précisément avec les pays à l'identification des bonnes pratiques pour anticiper l'évolution des besoins en compétences et élaborer des systèmes d'information qui permettront aux individus, aux employeurs, aux syndicats et aux institutions éducatives et de formation de mieux répondre à ces besoins. Par ailleurs, s'appuyant sur la Stratégie sur les compétences, l'OCDE aidera les pays à élaborer leur propre plan d'action national en faveur des jeunes.

L'OCDE lancera la première édition des Perspectives des compétences de l'OCDE en octobre 2013. Y seront présentés les premiers résultats de l'enquête de l'OCDE sur les compétences des adultes (PIAAC). Dans les années à venir, les Perspectives des compétences apporteront chaque année une perspective horizontale continue de l'OCDE sur le développement, l'activation et l'utilisation effective des compétences, exploitant à la fois des travaux d'analyse plus approfondis et les expériences nationales pour traiter des défis spécifiques en matière de compétences, par exemple améliorer la situation des jeunes.

La Stratégie sur les compétences est coordonnée par la Direction de l'éducation et des compétences, avec la Direction de l'emploi, du travail et des affaires sociales, en étroite collaboration avec LEED, le Centre de développement, le Centre de politique et d'administration fiscales, et d'autres directions selon les besoins. Le Secrétaire général adjoint Yves Leterme assure la supervision générale.

Pour plus d'informations, voir www.oecd.org/fr/edu/strategiedelocdesurlescompetences.htm et skills.oecd.org 


\section{L’Initiative sur la parité}

L'Initiative de l'OCDE sur la parité vise à renforcer l'égalité entre hommes et femmes en matière d'éducation, d'emploi et d'entrepreneuriat (les " trois E ") - les trois dimensions clés des opportunités économiques et sociales. L'égalité entre hommes et femmes n'est pas seulement une question d'autonomie économique ; c'est aussi une question de justice et d'équité qui comporte de multiples dimensions, politiques, éducatives, socioéconomiques et culturelles, à prendre en compte pour faire disparaître les disparités entre hommes et femmes. L'Initiative sur la parité adopte une approche horizontale, pangouvernementale, impliquant neuf directions et leurs différents comités, groupes de travail et organes directeurs.

L'Initiative de l'OCDE sur la parité montre clairement pourquoi les pays doivent agir dès maintenant pour tirer pleinement parti d'une plus grande égalité des chances entre les sexes. L'élévation du niveau de formation, en particulier chez les femmes, a stimulé la croissance économique au cours des 50 dernières années, et le comblement des écarts qui subsistent favorisera encore le développement. En outre, l'augmentation de la participation des femmes à la vie active jouera un rôle clé pour relever les défis liés au vieillissement de la population. L'Initiative sur la parité souligne aussi la précieuse contribution que l'entrepreneuriat des femmes peut apporter à la croissance économique dès lors qu'il bénéficie du soutien de mesures adéquates.

Les données observées, qui témoignent de l'existence d'un lien entre le congé parental, la garde des enfants et les disparités en matière d'emploi et de salaire, montrent que les politiques publiques ont un rôle crucial à jouer pour réduire les inégalités entre les sexes par rapport au travail rémunéré ou non, et pour traiter le problème de la surreprésentation des femmes dans les catégories les plus vulnérables des travailleurs informels, partout dans le monde.

La Recommandation sur la parité que les ministres adopteront lors de la RCM 2013 sera un instrument puissant pour faire évoluer les politiques publiques. Elle recense un certain nombre de mesures aptes à faire progresser l'égalité entre les sexes : garantir l'égalité d'accès à l'éducation, promouvoir des politiques et des conditions de travail qui facilitent la vie de famille, encourager la participation des pères au travail non rémunéré, s'efforcer d'instaurer un meilleur équilibre entre hommes et femmes dans les fonctions de direction, réduire les obstacles à l'entrepreneuriat des femmes, et aussi garantir l'égalité devant la loi et le plein respect des droits sociaux et politiques. L'Initiative sur la parité aide à orienter les débats nationaux, ainsi qu'en témoignent les demandes adressées à l'OCDE pour contribuer aux discussions sur les mesures à prendre, tant au niveau national que dans les instances internationales telles que le Parlement européen et la Commission européenne, par exemple. L'OCDE contribue aussi à l'effort international de collecte de données, en participant à l'Initiative faits et données sur l'égalité des sexes (EDGE), en coopération avec ONU Femmes et d'autres instances.

L'Initiative sur la parité est coordonnée par la Direction de l'emploi, du travail et des affaires sociales, sous la supervision du Secrétaire général adjoint Yves Leterme.

Pour plus d'informations, voir www.oecd.org/parite 


\section{La Stratégie pour le développement}

Le développement a toujours été au cœur de la mission de l'OCDE et, à l'occasion de la Réunion du Conseil au niveau des ministres (RCM) de 2011 qui a marqué le 50e anniversaire de l'Organisation, les ministres se sont félicités de la nouvelle approche globale du développement élaborée par l'OCDE. La Stratégie de l'OCDE pour le développement définit comment l'Organisation peut mettre en place et adapter des approches et des politiques analytiques en vue d'instaurer une croissance plus durable, plus forte et plus solidaire dans le plus grand nombre de pays possible.

Les membres et les pays partenaires ont recensé les domaines spécifiques dans lesquels l'OCDE peut apporter une valeur ajoutée, qu'il s'agisse des Examens multidimensionnels par pays, de la fiscalité et de la mobilisation des ressources intérieures, de l'égalité entre les sexes, des migrations, de l'innovation, de l'éducation ou encore de la croissance verte inclusive. Ces projets viennent aussi renforcer les travaux de l'OCDE sur la Cohérence des politiques au service du développement et le Partage des connaissances, ainsi que la contribution de l'Organisation aux processus mondiaux tels que la définition du programme d'action pour l'après-2015 et les travaux du G20.

L'éventail des progrès accomplis l'année dernière donnent la mesure de l'ampleur des projets qui ont été engagés. Parce que l'association de pays développés et en développement autour d'un programme d'action commun favorise le partage des connaissances, deux réseaux innovants de partage des politiques ont été créés - ayant trait respectivement aux chaînes de valeur mondiales et aux industries extractives -, ainsi qu'une Alliance pour le partage des connaissances. Pour étayer la formulation de politiques fondées sur des données probantes, de nouvelles analyses, de nouvelles approches et de nouveaux outils vont être déployés et expérimentés après avoir fait l'objet de projets pilotes. Un récent rapport de l'OCDE intitulé Illicit Financial Flows from Developing Countries: Measuring OECD Responses, mettra en évidence des lacunes dans la façon dont les pays de l'OCDE appliquent les normes internationales. Les travaux sur la Cohérence des politiques au service du développement et la Sécurité alimentaire dans le monde recensent divers moyens de mettre en œuvre des réformes et d'assurer une action concertée à l'échelle mondiale. L'approche retenue dans le cadre des Examens multidimensionnels par pays, qui permet de prendre simultanément en compte de multiples objectifs de développement, est actuellement mise en application dans plusieurs pays en développement comme les Philippines, le Myanmar et l'Uruguay. Dans le secteur de l'éducation, un projet pilote a pour objet d'adapter un outil mesurant la qualité de l'éducation de façon à en rehausser la pertinence pour un plus large éventail de pays en développement. D'autres leviers d'action, tels que la mobilisation des ressources intérieures et la lutte contre les flux illicites, se traduisent déjà par des résultats concrets dans les pays partenaires, où ils prennent la forme d'un accroissement des recettes fiscales collectées à l'issue de vérifications des prix de transfert d'entreprises multinationales ou de réformes législatives ou réglementaires applicables aux incitations fiscales et à l'échange de renseignements entre pays.

La Stratégie pour une croissance verte et la Stratégie pour la parité ont déjà permis de renforcer les synergies et la cohérence. D'autres mesures seront prises pour identifier les synergies avec d'autres stratégies et initiatives élaborées par l'Organisation (par exemple NAEC, Croissance inclusive, Stratégie sur les compétences, Plan d'action pour les jeunes). Dans cette optique, il s'agira de veiller à la pertinence des concepts, actuels et en devenir, tant pour les membres que pour les économies en développement et émergentes. Nous examinerons comment l'OCDE travaille avec des pays et régions partenaires à différents stades de développement, en renforçant son rôle de plateforme de production et de partage des connaissances, et en modernisant ses dispositifs de suivi et de mesure.

La supervision de la Stratégie pour le développement est assurée par le Secrétaire général adjoint Rintaro Tamaki.

Pour plus d'informations, voir www.oecd.org/fr/pcd/towardsanoecdstrategyondevelopment.htm 


\section{L’Initiative du vivre mieux}

Depuis de nombreuses décennies, le produit intérieur brut est le principal indicateur utilisé par les institutions nationales et internationales pour définir et mesurer le progrès. Mais un indicateur ainsi focalisé sur la croissance économique ne rend pas compte des multiples facteurs qui affectent la vie des gens. Au cours de la dernière décennie, plusieurs pays et institutions ont entrepris d'identifier d'autres façons de mesurer le progrès des sociétés. Lancée en 2011 et s'inscrivant dans le contexte des efforts déployés au niveau international pour aller au delà du PIB, l'Initiative du vivre mieux introduit un cadre OCDE pour analyser le bien-être et le progrès des sociétés.

Le rapport Comment va la vie?, également lancé en 2011, rassemble pour la première fois des mesures du bien-être comparables au niveau international, qui prennent en compte les conditions de vie matérielles et la qualité de vie des personnes selon onze dimensions : revenu et patrimoine, emploi et gains, logement, santé, équilibre entre travail et vie de famille, éducation et compétences, relations sociales, engagement citoyen et gouvernance, qualité de l'environnement, sécurité et bien-être subjectif. La deuxième édition de Comment va la vie? sera publiée d'ici la fin de l'année.

Également créé il y a un peu plus d'un an dans le cadre de l'Initiative, l'Indicateur du vivre mieux de l'OCDE est une plateforme en ligne interactive qui invite le public à visualiser la situation en termes de bien-être dans les pays de l'OCDE, au regard des critères que chacun juge les plus importants. À ce jour, l'Indicateur du vivre mieux a attiré plus de 1,5 million d'utilisateurs originaires de plus de 180 pays. Actualisé chaque année, il a été étendu en 2012 au Brésil et à la Fédération de Russie et s'est enrichi d'une version française et d'une version russe.

L'OCDE s'emploie actuellement à lier la mesure du bien-être aux politiques publiques. Cela implique, dans un premier temps, d'identifier les synergies et les choix à faire entre différentes dimensions du bien-être. Les travaux de l'OCDE ont montré, par exemple, que les inégalités de revenu se sont accentuées, engendrant, dans certains pays, des tensions sociales auxquelles les pouvoirs publics doivent répondre. Les résultats en termes de bien-être diffèrent également selon que l'on évoque la situation des hommes ou celle des femmes. L'OCDE prend en compte la dimension hommes-femmes dans l'Indicateur du vivre mieux et présente des informations complètes sur son portail dédié à la parité, qui s'inscrit dans l'ensemble des travaux que l'Organisation consacre à cette problématique.

Il est difficile pour les analystes et les dirigeants d'appréhender les multiples dimensions du bien-être. L'enjeu est de comprendre comment les politiques macroéconomiques et sectorielles influent sur les divers aspects du bien-être, afin d'en déduire des messages pratiques à l'intention des dirigeants. Les travaux de l'OCDE en ce sens ont commencé, dans le contexte des examens par pays, dans le contexte du projet de l'Organisation sur une croissance inclusive et dans le cadre de l'initiative NAEC.

Les travaux sur la mesure du bien être sont coordonnés par la Direction des statistiques.

Pour plus d'informations, voir www.oecd.org/fr/statistiques/initiativeduvivremieuxmesurerlebien-etreetleprogrès.htm

L'Indicateur du vivre mieux est coordonné par la Direction des relations extérieures et de la communication.

Pour plus d'informations, voir www.oecdbetterlifeindex.org/fr 


\section{Direction de la coopération pour le développement}

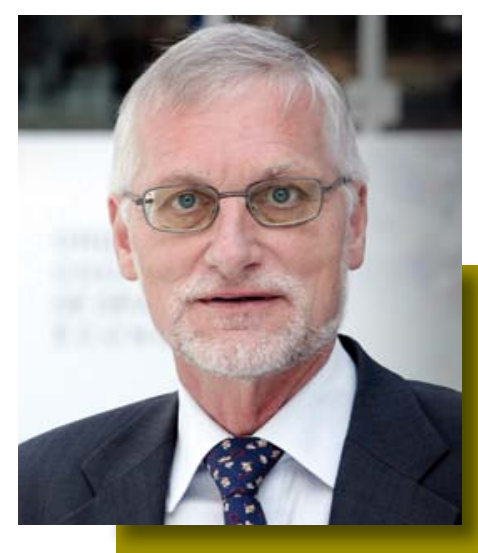

Jon Lomoy

Directeur

Courriel : dac.contact@oecd.org

« Le monde change et nous

changeons également pour

améliorer la coopération

internationale pour le

développement. Ce qui

signifie : faire les bons choix

de financement - élargir les

sources de financement et

mieux utiliser l'aide ; mener les

bonnes politiques - construire

des partenariats judicieux,

puiser dans les richesses

de l'OCDE et mobiliser ses

réseaux de décideurs au service

du développement ; et bien

orienter la coopération pour le

développement - par les examens

par les pairs et une démarche

axée sur les résultats. 》)
Dans le contexte de la contraction de l'aide publique au développement, la réflexion porte désormais sur les objectifs de développement non encore réalisés - les OMD - et sur le cadre de la coopération pour le développement après 2015. Il est de plus en plus clair que l'aide n'est pas la panacée. La clé du succès résidera dans la mise en place d'initiatives et de mécanismes beaucoup plus larges pour financer et promouvoir le développement. À l'horizon 2015, la pauvreté demeurera évidemment une préoccupation majeure : malgré des avancées notables pour réduire de moitié la pauvreté, nous sommes loin d'avoir atteint le but global de l'éradication de l'extrême pauvreté. Parallèlement, les solutions apportées au problème de la pauvreté devront tenir compte, pour être durables, de la viabilité environnementale, l'éducation, l'équité sociale et la croissance verte, ainsi que d'autres facteurs complexes. Soucieuse d'apporter un éclairage sur un défi présentant de multiples facettes, la Direction de la coopération pour le développement a progressé, en 2012, vers l'intégration dans le développement des principes de la croissance verte. Nous exerçons un suivi des divers apports dont bénéficie le développement, notamment grâce à l'élaboration de notre Système de notification des pays créanciers et à la publication de notre rapport annuel sur l'aide multilatérale. Nous étudions en outre - en coopération avec le Centre de politique et d'administration fiscales de l'OCDE - les flux de capitaux illicites dans les pays en développement, ainsi que les mesures susceptibles d'être prises par les donneurs pour combattre ce fléau. Nombre de ces questions seront traitées lors du prochain Forum mondial sur le développement et dans notre Rapport 2013 sur la coopération pour le développement.

La Direction de la coopération pour le développement aide le Comité d'aide au développement (CAD) à élaborer des politiques meilleures pour une vie meilleure en examinant le financement du développement, en s'assurant que les investissements sont efficaces et en encourageant les bonnes politiques.

Grâce à des partenariats inclusifs au service du développement, elle constitue un forum ouvert à tous pour partager les bonnes pratiques et améliorer les connaissances. 


\section{Activités principales}

- Architecture et financement de l'aide : www.oecd.org/cad/architecture

- Efficacité de l'aide : www.oecd.org/cad/efficacite

- Aide pour le commerce : www.oecd.org/cad/apc

- Statistiques de l'aide : www.oecd.org/cad/stats

- Conflits et situations de fragilité : www.oecd.org/cad/incaf

- Ouverture : www.oecd.org/cad/portesouvertes

- Environnement et développement : www.oecd.org/cad/environnement

- Évaluation des programmes de développement : www.oecd.org/cad/evaluation

- Égalité homme-femme et développement : www.oecd.org/cad/genre

- Gouvernance et développement : www.oecd.org/cad/gouvernance

- Examens par les pairs des efforts des membres du CAD : www.oecd.org/cad/examens-pairs

- Statistiques du développement : www.paris21.org

\section{Publications principales}

- Réduction de la pauureté et croissance pro-pauures : Le rôle de l'autonomisation

- L'efficacité de l'aide dans le secteur de la santé : Progrès et enseignements

- Improving International Support to Peace Processes: The Missing Piece

- L'architecture de l'aide au développement 2012

- Evaluating Peacebuilding Activities in Settings of Conflict and Fragility - Improving Learning for Results

- Trois volumes de la série Lessons from Peer Reviews Series : Towards Better Humanitarian Donorship, Supporting Partners to Develop their Capacity, et Partnering with Civil Society

- Coopération pour le développement 2012 : Comment intégrer durabilité et développement

- Tax and Development: Aid modalities for Strengthening Tax Systems

- Fragile States: Resource Flows and Trends

- Croissance verte et pays en développement

\section{Manifestations principales}

- Forum mondial sur la gouvernance publique : Une meilleure gouvernance pour une croissance inclusive, Paris, 21 novembre 2012

- Forum mondial de l'OCDE sur l'agriculture : Cohérence des politiques de sécurité alimentaire dans les pays en développement, Paris, 26 novembre 2012

- Réunion à haut niveau du CAD 2012, Londres, 4-5 décembre 2012 (lancement du Rapport de la coopération pour le développement 2012)

- Atelier régional BAfD-OCDE « Croissance verte en Afrique : concepts, outils et stratégies pour bâtir des économies plus vertes et un avenir durable », Lusaka, 15-18 janvier 2013

- Dialogue sur l'aide pour le commerce 2013, Paris, 16-17 janvier 2013

- Forum mondial sur le développement 2013, Paris, 4-5 avril 2013 


\section{Département des affaires économiques}

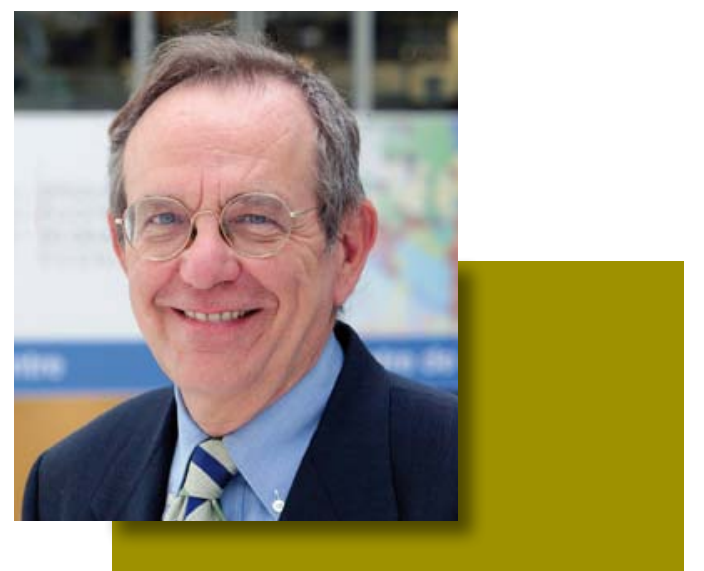

Pier Carlo Padoan

Secrétaire général adjoint

et Chef économiste

Courriel : eco.contact@oecd.org

« Après cinq années de crise,

l'économie mondiale a de

nouveau fléchi vers la fin de

2012, sous l'effet d'une nette

dégradation de la confiance.

Ce déficit de confiance tient à

des mesures de court terme

inefficaces ainsi qu'à un

manque de stratégies crédibles

à long terme. Face à la crise,

les pouvoirs publics devraient

recourir à tous les instruments

d'action disponibles : monétaires,

financiers, budgétaires et

structurels. Le Département

des affaires économiques peut

apporter sa pierre à l'édifice en

faisant bénéficier les autorités

de ses recommandations

extrêmement utiles, fondées

sur des analyses économiques

solides. $\gg$
En 2012, le Département des affaires économiques (ECO) a axé ses recommandations sur la promotion des réformes structurelles qui, à l'heure où les politiques macroéconomiques sont mises à rude épreuve, sont devenues l'instrument d'action de dernier ressort. L'importance des réformes structurelles tient à la fois à une raison conventionnelle, qui est leur effet stimulant sur le bien-être et la croissance à long terme, mais aussi à leur capacité d'alléger en partie les pressions qui s'exercent sur les politiques macroéconomiques. De meilleures politiques structurelles contribueront à assurer la viabilité des finances publiques et à élargir les marges de manœuvre des autorités monétaires. Si elles sont judicieusement conçues, elles peuvent également favoriser une croissance inclusive et verte. Les réformes structurelles peuvent en outre renforcer la confiance, ce qui est un point essentiel. Pour ces raisons, elles constituent plus que jamais une priorité pour l'OCDE. Elles figurent en bonne place dans les plans d'action et les programmes de travail du G20, auxquels ECO continuera de contribuer largement en vue d'assurer une croissance mondiale forte, durable et équilibrée.

Au cours des 12 derniers mois, ECO a publié 23 études par pays, notamment sur l'Indonésie, pays qui bénéficie de l'engagement renforcé de l'Organisation. Les travaux continuent dans les domaines liés aux missions confiées par le G20 à l'OCDE. En 2012, en sus de ses publications phares, les Perspectives économiques de l'OCDE et Objectif croissance, ECO a lancé une nouvelle série, les Études de politique économique de l'OCDE, destinée à diffuser certaines études sur des questions d'actualité liées aux politiques structurelles et macroéconomiques. Au cours de l'année 2012 ont été publiés trois numéros de cette série, consacrés à des scénarios de croissance à long terme de l'économie mondiale à l'horizon 2060, aux facteurs de vulnérabilité liés à la mobilité internationale des capitaux, et à l'assainissement budgétaire à la suite de la crise. Le Département des affaires économiques a également publié 85 documents de travail en 2012, dont le $1000^{\circ}$ document de cette série, dans lequel étaient exposés les scénarios à long terme à l'horizon 2060.

Le Département des affaires économiques assure un suivi des politiques économiques multilatérales, nationales et structurelles pour les membres de l'OCDE ainsi que pour les pays en voie d'adhésion et bénéficiant de l'engagement renforcé. 


\section{Activités principales}

- Études économiques par pays : www.oecd.org/fr/eco/etudes

- Analyses et conseils en matière de politiques structurelles pour l'ensemble des pays : www.oecd.org/economie/objectifcroissance

- Perspectives économiques : www.oecd.org/perspectiveseconomiques

- Notes de politique économique du Département des affaires économiques : www.oecd.org/fr/economie/monetaire/notesdepolitiqueeconomiquedudepartementdesaffaireseconomiques.htm

- Documents de travail du Département des affaires économiques : www.oecd.org/fr/eco/documentsdetravaildudepartementdesaffaireseconomiques.htm

- Études de politique économique du Département des affaires économiques : www.oecd.org/fr/eco/economicpolicypapers.htm

- Questions monétaires et financières : www.oecd.org/fr/eco/monetaire

- Finances publiques : www.oecd.org/fr/eco/finances-publiques

\section{Publications principales}

- Réformes économiques - Objectif croissance 2013

- Perspectives économiques de l'OCDE

- Études économiques de l'OCDE (portant sur les pays membres et certains pays non membres)

- Revue économique de l'OCDE

- Promoting Inclusive Growth: Challenges and Policies

\section{Manifestations principales}

- Lancement de l'édition 2013 d'Objectif croissance, Moscou, 15 février 2013

- Lancement des Perspectives économiques intermédiaires relatives aux grandes économies mondiales, Paris, 28 mars 2013

- Lancement des Perspectives économiques de l'OCDE, Paris, 28-29 mai 2013 


\section{Direction de l'éducation et des compétences}

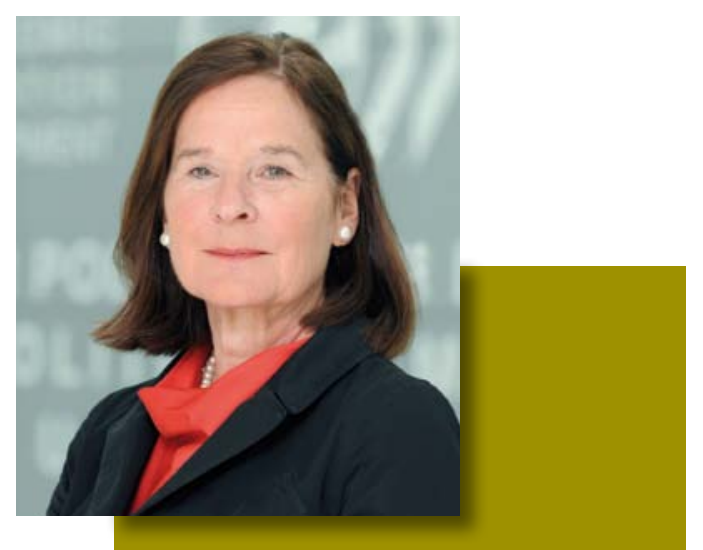

Barbara Ischinger

Directrice

Courriel : edu.contact@oecd.org

« Les compétences changent

la vie et entraînent l'économie.

Elles déterminent en effet de plus

en plus les résultats en termes

d'emploi et la participation

sociale. Si nous ne parvenons

pas à adapter le savoir et les

compétences des individus à

l'évolution des exigences des

emplois et des professions,

nous en subirons tous les

conséquences à la fois dans le

court terme, sous la forme de

pénuries de compétences, et

dans le long terme, sous la forme

d'effets négatifs sur la croissance

économique et l'égalité des

chances. $\gg$
En mai 2012, l'OCDE a présenté sa "Stratégie sur les compétences » lors de la Réunion du Conseil au niveau des ministres. Cette initiative transversale, la plus ambitieuse menée en 2012 par la Direction de l'éducation et des compétences, soutiendra les efforts des pays pour promouvoir le progrès social et une croissance économique durable en améliorant les résultats scolaires, en stimulant le capital humain et en dynamisant l'emploi. Afin d'aider les pays à obtenir une image plus claire des compétences de leur population active et à se situer par rapport aux autres pays, l'OCDE réalise l'enquête internationale la plus complète jamais menée sur les compétences des adultes. Il faut pour cela tester plus de 5000 personnes de 16 à 65 ans dans chacun des 26 pays participants. Les premiers résultats de l'Enquête de l'OCDE sur les compétences des adultes seront publiés en octobre 2013 lors du lancement du OECD Skills Outlook.

Le Programme international pour le suivi des acquis des élèves (PISA) montre l'efficacité de l'éducation dans les différents pays, considérés isolément et par rapport aux autres. Les résultats de l'enquête de 2012 seront publiés en décembre 2013. Le cycle PISA 2012 portait sur les compétences en mathématiques des élèves de 15 ans et sur l'évaluation de leurs compétences en compréhension de l'écrit et en sciences.

La Direction de l'éducation et des compétences conduit les travaux de l'OCDE visant à aider les pays membres et non membres à assurer à tous des acquis scolaires de haute qualité, à concevoir des politiques plus efficaces en faveur des compétences et à renforcer ainsi l'emploi et la croissance. Nous ne saurions trop souligner que l'acquisition et la mise à jour des compétences doivent se poursuivre tout au long de la vie. Le Comité des politiques d'éducation fixe l'orientation stratégique et supervise les travaux de l'OCDE dans le domaine de l'éducation et des compétences. 


\section{Activités principales}

- Centre pour la recherche et l'innovation dans l'enseignement (CERI) : www.oecd.org/fr/sites/educeri

- Éducation et accueil des jeunes enfants : www.oecd.org/edu/earlychildhood

- Indicateurs de l'enseignement : www.oecd.org/fr/edu/rse2012.htm

- Blog Educationtoday : http://loecdeducationtoday.blogspot.com

- Équité dans l'enseignement : www.oecd.org/edu/equite

- Cadres d'évaluation en vue d'améliorer les résultats scolaires : www.oecd.org/edu/evaluationpolicy

- Équipements et infrastructures de l'enseignement : www.oecd.org/fr/edu/etudes-pays/centrepourdesenvironnementspedagogiquesefficacescele/

- Étude de faisabilité concernant l'évaluation des résultats de l'enseignement supérieur (AHELO) : www.oecd.org/edu/ahelo-fr

- Programme international pour l'évaluation des compétences des adultes (PIAAC) : www.oecd.org/site/piaac

- Stratégie de l'OCDE sur les compétences : http://skills.oecd.org

- Programme international pour le suivi des acquis des élèves (PISA) : www.oecd.org/pisa/pisaenfranais

- Enquête internationale sur l'enseignement et l'acquisition de connaissances (TALIS) : www.oecd.org/edu/talis-fr

- Programme sur la gestion des établissements d'enseignement supérieur (IMHE) : www.oecd.org/fr/sites/eduimhe

\section{Publications principales}

- Des compétences meilleures pour des emplois meilleurs et une vie meilleure: Une approche stratégique des politiques sur les compétences

- L'éducation aujourd'hui 2013 : La perspective de l'OCDE

- Grade Expectations: How Marks and Education Policies Shape Students' Ambitions

- OECD Reviews of Evaluation and Assessment in Education: Synergies for Better Learning: An International Perspective on Evaluation and Assessment

- Lisons-leur une histoire! Le facteur parental dans l'éducation

- Teachers for the 21st Century: Using Evaluation to Improve Teaching

- Teaching Practices and Pedagogical Innovations: Evidence from TALIS

- The Experience of New Teachers: Results from TALIS 2008

- Les grandes mutations qui transforment l'éducation 2013

\section{Manifestations principales}

- $15^{\mathrm{e}}$ séminaire OCDE/Japon : "Global Strategies for Higher Education Global Trends and Rethinking the Role of Government », 6-7 février 2013

- Sommet international 2013 sur la profession enseignante, Amsterdam, 13-14 mars 2013

- Lancement de Regards sur l'éducation 2013, Bruxelles, 25 juin 2013

- Réunion informelle des ministres de l'Éducation : "Fostering Skills and Employability through Education, Istanbul ", 2-3 octobre 2013

- Lancement de OECD Skills Outlook, Bruxelles, 8 octobre 2013

- Publication des résultats du cycle 2012 du Programme international pour le suivi des acquis des élèves (PISA), Washington DC, 3 décembre 2013 


\section{Direction de l'emploi, du travail et des affaires sociales}

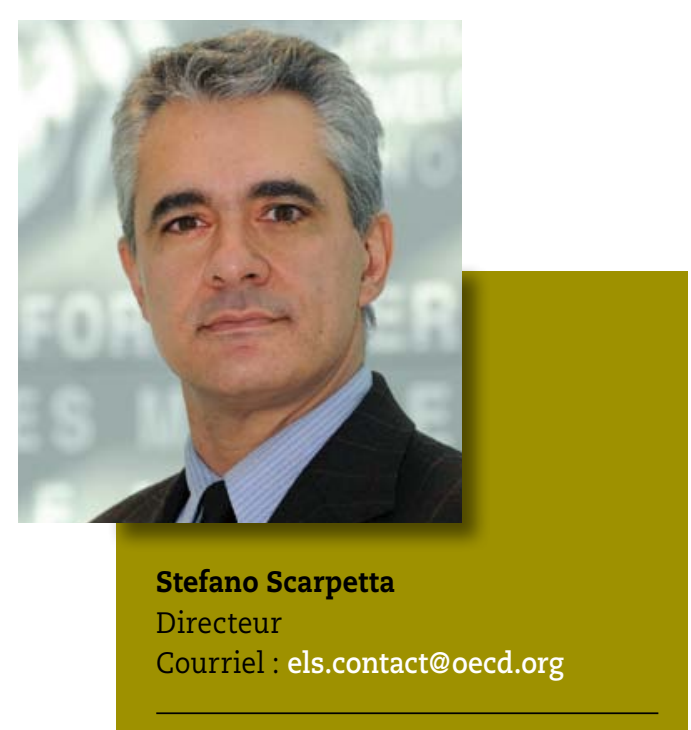

« Les temps sont durs pour les décideurs publics, confrontés à de nombreux problèmes : reprise lente et inégale, persistance de taux de chômage élevés et sévères contraintes budgétaires. Les pouvoirs publics doivent poursuivre la mise en œuvre de réformes structurelles ambitieuses tout en investissant dans des politiques du marché du travail efficaces par rapport à leur coût, qui soutiennent les chômeurs tout en maintenant les incitations au travail. C'est ainsi qu'ils pourront combattre le chômage de longue durée, dynamiser la création d'emplois et améliorer les perspectives des jeunes. $\gg$
En 2012, l'OCDE a aidé les pays à trouver de meilleures solutions pour gérer l'impact de la crise sur les emplois et les revenus. Les Perspectives de l'emploi de l'OCDE 2012 analysent les politiques soutenant les chômeurs tout en encourageant leur rapide retour vers l'emploi. L'OCDE a aussi activement contribué à établir les documents de référence pour la troisième réunion des ministres du Travail des pays du G20 en mai. L'OCDE a par ailleurs fait paraître un rapport novateur intitulé Inégalités hommes-femmes : Il est temps d'agir. Les travaux actuels portent également sur les mesures d'insertion des immigrés, avec la publication Trouver ses marques : Les indicateurs de l'OCDE sur l'intégration des immigrés 2012, qui présente la première comparaison internationale de l'intégration économique et sociale des immigrés et de leurs enfants dans les pays de l'OCDE. De nombreux pays bénéficient de conseils ciblés sur la réforme de leur système de santé, en particulier grâce aux Reviews of Health Care Quality: Raising Standards de l'OCDE. Health at a Glance: Asia/Pacific 2012, une nouvelle publication réalisée en collaboration avec l'Organisation mondiale de la santé, présente les plus récentes tendances comparables sur des aspects clés de la santé et des systèmes de santé dans certains pays et économies de la région Asie/Pacifique. Dans les années à venir, l'OCDE continuera de centrer ses activités relatives aux problèmes de l'emploi, du travail et aux problèmes sociaux sur les moyens de concilier les pressions à court terme dues à la crise et à la faiblesse de la reprise et les problématiques à long terme du vieillissement de la population et de la mondialisation. Il faut faire davantage, en particulier, pour améliorer les perspectives professionnelles des jeunes et promouvoir l'égalité des chances. Dans le domaine de la santé, l'amélioration de la prévention et de la prise en charge des maladies chroniques sera au cœur des travaux, avec des rapports novateurs sur l'alcool et le cancer.

La Direction de l'emploi, du travail et des affaires sociales (ELS) de l'OCDE guide les travaux de l'Organisation en matière d'emploi, de politiques sociales, de migration internationale et de santé. Elle supervise les travaux portant sur des domaines interdépendants de l'action publique afin d'aider les pays à développer l'emploi et à améliorer le bien-être social et la santé dans un contexte de vieillissement de la population, de mondialisation et de rapide progrès technologique. 


\section{Activités principales}

- Politiques et données sur l'emploi : www.oecd.org/fr/els/emp

- Politiques et données sur la santé : www.oecd.org/fr/els/systemes-sante

- Politiques et données sociales : www.oecd.org/fr/els/soc

- Familles et enfants : www.oecd.org/fr/els/famille

- Régime de pensions : www.oecd.org/fr/els/pensions-publiques

- Politiques et données des migrations internationales : www.oecd.org/fr/els/mig

\section{Publications principales}

- Inégalités hommes-femmes : Il est temps d’agir 2012

- Resserrer les liens avec les diasporas : Panorama des compétences des migrants 2012

- Health at Glance: Asia/Pacific 2012, Europe 2012

- Base de données de l'OCDE sur la santé 2013

- A Good Life in Old Age? Monitoring and Improving Quality in Long-term Care 2013

- Strengthening Social Cohesion in Korea 2013

- Perspectives des migrations internationales de l'OCDE 2013

- Santé mentale et emploi : Belgique, Danemark, Norvège, Suède 2013

- Perspectives de l'emploi de l'OCDE 2013

\section{Manifestations principales}

- Réunion des Correspondants nationaux de la Base de données de l'OCDE sur la santé, 11-12 octobre 2012

- Forum de l'OCDE sur la parité, Paris, 17 décembre 2012

- Séminaire pour le lancement de Santé mentale et emploi : Belgique, Bruxelles, 27 janvier 2013

- Séminaire pour le lancement de Santé mentale et emploi : Danemark, Copenhague, 25 février 2013

- Séminaire pour le lancement de Santé mentale et emploi : Norvège, Oslo, 5 mars 2013 


\section{Centre pour l'entrepreneuriat, les PME et le développement local}

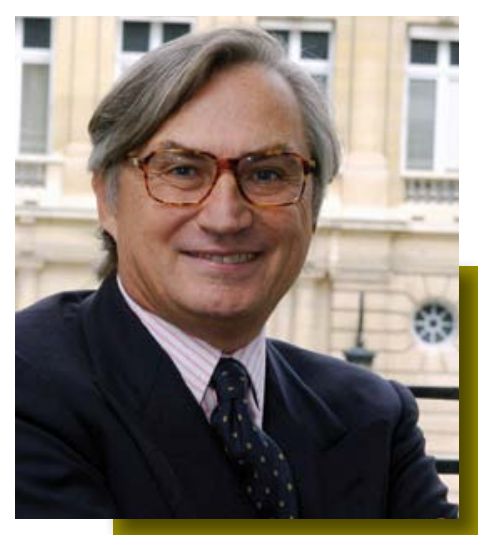

\section{Sergio Arzeni}

Directeur

Courriel : cfe.contact@oecd.org

\section{« L'économie mondiale doit}

reposer sur des fondements plus

solides. Il faut que les petites

entreprises et les entrepreneurs

puissent jouer leur rôle dans

la croissance, l'emploi et

l'inclusion sociale. Pour cela,

ils ont besoin des compétences

appropriées et des financements

pour mettre en œuvre leur

stratégie d'entreprise. Il faut

adopter une approche intégrée

de l'emploi, de l'entrepreneuriat,

des compétences et du

développement économique afin

d'élaborer des stratégies efficaces

de création d'emplois. 》)
Le Centre pour l'entrepreneuriat, les PME et le développement local (CFE) aide les pays à concevoir et mettre en œuvre des politiques efficaces pour l'emploi local et le développement économique, les PME et l'entrepreneuriat, ainsi que le tourisme. Il mène des examens par les pairs au niveau local et national, et contribue aux projets de l'OCDE concernant les Nouvelles approches face aux défis économiques, la croissance inclusive, les compétences, la croissance verte et la parité. Le CFE s'appuie aussi sur les compétences de son vaste réseau d'experts internationaux et organise des consultations régulières avec la société civile.

En 2013, le CFE continuera d'élargir son Tableau de bord sur le financement des PME et de l'entrepreneuriat. Il lancera également de nouveaux travaux sur les instruments de financement alternatifs pour les PME et sur les compétences au service de l'innovation dans les PME, et examinera les opportunités de création d'entreprise offertes aux chômeurs, aux personnes handicapées et aux groupes ethniques minoritaires.

Durant l'année à venir, le CFE rassemblera un ensemble d'indicateurs sur les capacités des pays à créer des emplois plus nombreux et de meilleure qualité en menant des politiques actives et efficaces du marché du travail et des politiques innovantes en matière d'entrepreneuriat au niveau local. Le Centre élaborera des stratégies transversales pour l'emploi des jeunes ainsi que des stratégies territorialisées pour lutter contre l'exclusion du marché du travail en une période de ressources limitées, et il examinera les moyens de développer des compétences vertes pour faciliter le passage à une économie sobre en carbone. Les travaux viseront aussi à aider les collectivités à assurer un développement local efficace et à renforcer leurs capacités par le biais de l'apprentissage entre pairs et de forums d'échanges, notamment au Centre du programme LEED pour le développement local de Trente.

Les autres travaux consisteront à examiner des politiques du tourisme efficaces au service de la croissance, une attention particulière étant accordée aux politiques et aux pratiques favorisant une croissance plus verte, plus forte et plus inclusive. Le CFE collaborera également avec les pouvoirs publics et les professionnels du tourisme pour promouvoir des emplois de qualité dans ce secteur, examinera l'impact économique du tourisme au niveau infranational et passera en revue les politiques et pratiques relatives à la fiscalité du tourisme, aux activités touristiques et à l'économie créative. 


\section{Activités principales}

- Programme pour le développement économique et la création d'emplois au niveau local (LEED) : www.oecd.org/fr/cfe/leed

- PME et entrepreneuriat : www.oecd.org/fr/cfe/pme

- Tourisme : www.oecd.org/fr/cfe/tourisme

\section{Publications principales}

- Clean-Tech Clustering as an Engine for Local Development in Israel

- Demographic Change and Local Development: Shrinkage, Regeneration and Social Dynamics

- Le financement des PME et des entrepreneurs 2013 : Tableau de bord de l'OCDE

- Green Entrepreneurship, Eco-Innovation and SMEs

- Tendances et politiques du tourisme de l'OCDE 2012

- Promouvoir l'entrepreneuriat dans les universités tunisiennes

- Skills Development and Training in SMEs

- Skills for Growth: Implementing Locally

- Pallier la pénurie d'entrepreneurs : Politiques d'entrepreneuriat inclusif en Europe

\section{Manifestations principales}

- Neuvième réunion annuelle du Forum sur les partenariats et la gouvernance locale - Mettre en œuvre le changement: Un nouvel agenda local pour l'emploi et la croissance, Dublin-Kilkenny, 26-27 mars 2013

- Quatrième université d'été internationale sur le développement local en Amérique latine, Trente, Italie, 15-25 juillet 2013 


\section{Direction de l'environnement}

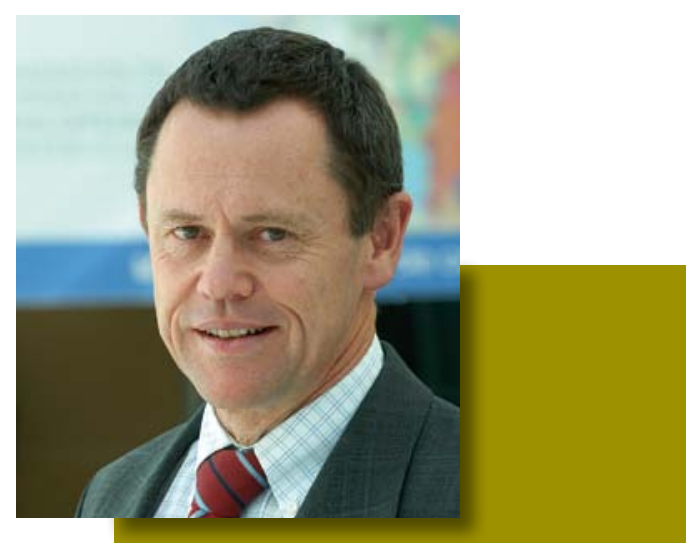

Simon Upton

Directeur

Courriel : env.contact@oecd.org

\section{« En période de difficultés}

économiques, il est

compréhensible que les pouvoirs

publics et les entreprises

adoptent des vues à court terme.

Mais pour de nombreux défis

environnementaux, cette attitude

sera très coûteuse à long terme,

notamment si les investissements

de longue durée dans les

infrastructures ne tiennent pas

compte de l'impact et des coûts

du changement climatique à

l'avenir. Nous renforçons notre

capacité d'analyse économique

et environnementale à long

terme pour aider les pays à

mieux comprendre les avantages

économiques de l'action

environnementale. »)
Les Perspectives de l'environnement de l'OCDE à l'horizon 2050 et le rapport Croissance verte et pays en développement sont des contributions majeures de l'OCDE à la Conférence des Nations Unies sur le développement durable (Rio+20) de juin 2012. En septembre 2012, une Table ronde sur la mobilisation de l'investissement privé au service d'infrastructures sobres en carbone et résilientes au changement climatique a rassemblé des responsables politiques et des experts pour examiner la meilleure façon d'intégrer les politiques d'investissement et celles liées au changement climatique afin de mettre en place un cadre d'action cohérent, stable et durable. La COP-18 de la Convention-cadre sur les changements climatiques à Doha, en novembre-décembre 2012, a été un événement crucial dans la lutte contre le changement climatique. Des études de l'OCDE sur le sujet ont été mises en relief dans plusieurs manifestations parallèles. Un séminaire a été organisé conjointement par l'OCDE et le Commissariat général au développement durable (France) pour examiner le lien entre l'évaluation des services écosystémiques et la conception ainsi que l'application de politiques publiques aux niveaux national et local. Les examens environnementaux de l'OCDE sur l'Italie, le Mexique, l'Autriche, l'Afrique du Sud et la Colombie seront également préparés ou publiés en 2013.

La Direction de l'environnement aide les pays à concevoir et appliquer des politiques efficaces et efficientes pour répondre aux problèmes d'environnement et gérer durablement les ressources naturelles. Elle analyse les relations entre l'environnement et les préoccupations économiques, sectorielles ou sociales, dans des domaines tels que le changement climatique; la biodiversité ; l'eau; les déchets; la fiscalité écologique ; la sécurité des produits chimiques ; les nanomatériaux et les cultures transgéniques ; l'agriculture ; les transports ; les échanges ; l'investissement ; et le développement. 


\section{Activités principales}

- Biodiversité : www.oecd.org/env/biodiversite

- Changement climatique : www.oecd.org/fr/env/cc/

- Politique de l'environnement et innovation technologique :

www.oecd.org/fr/env/consommation-innovation/politiquedelenvironnementetinnovationtechnologique.htm

- Examens environnementaux par pays : www.oecd.org/fr/env/examens-pays

- Données et indicateurs environnementaux : www.oecd.org/fr/env/indicateurs-modelisation-perspectives

- Modélisation économique-environnementale : www.oecd.org/environnement/modelisation

- Perspectives de l'environnement de l'OCDE : www.oecd.org/environnement/perspectives

- Outils et évaluation des politiques de l'environnement : www.oecd.org/fr/environnement/outils-evaluation/

- Politique d'environnement et comportement des ménages : www.oecd.org/fr/env/consommation-innovation/menages.htm

- Croissance verte : www.oecd.org/fr/croissanceverte

- Productivité des ressources et déchets : www.oecd.org/fr/env/dechets

- Sécurité des produits chimiques et biosécurité : www.oecd.org/fr/securitechimique

- Eau : www.oecd.org/eau

\section{Publications principales}

- Prix effectifs du carbone

- Politique énergétique et climatique : Infléchir la trajectoire technologique

- Panorama de l'environnement 2013

- Les mécanismes de financement pour la biodiversité

- Examens environnementaux de l’OCDE : Allemagne 2012, Slovénie 2012, Mexique 2013, Italie 2013, Autriche, Afrique du Sud, Colombie

- Politique de l'environnement et comportement des ménages : Les résultats de la deuxième enquête

- Le commerce illicite de produits écologiquement sensibles

- Material Resources, Productivity and the Environment

- Sustainable Materials Management: Making Better Use of Resources

- L'eau et l'adaptation au changement climatique : Les politiques pour naviguer en eaux troubles

- Water Security: Managing Risks, Improving Resilience

\section{Manifestations principales}

- Forum sur la croissance verte et le développement durable, Paris, 23 novembre 2012

- Séminaire sur l'évaluation des services rendus par les écosystèmes et son utilisation dans les politiques publiques, Paris, $1^{\mathrm{er}}$ février 2013

- Deuxième conférence annuelle de la Plateforme de connaissances sur la croissance verte (GGKP), Paris, 4-5 avril 2013

- Forum mondial de l'OCDE sur l'environnement : Les instruments économiques au service de la gestion des matières, Paris, du 2 au 6 décembre 2013 


\section{Direction des affaires financières et des entreprises}

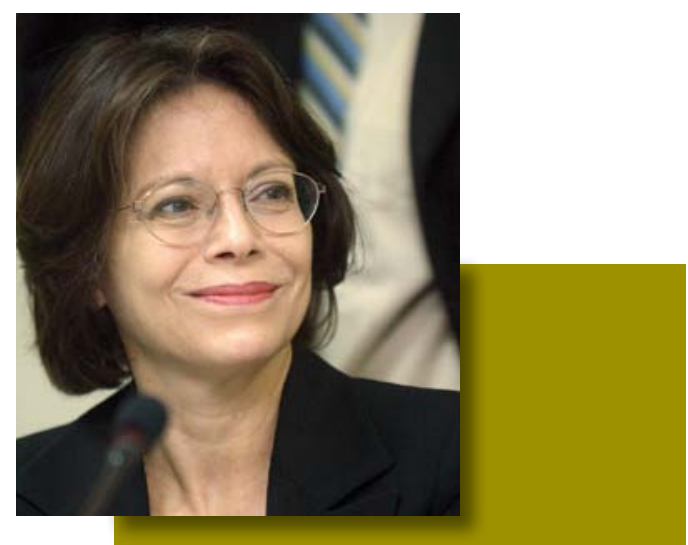

Carolyn Ervin

Directrice

Courriel : daf.contact@oecd.org

« L'OCDE fait tout son possible pour aider les gouvernements à honorer leurs engagements en matière d'ouverture des marchés et à favoriser l'intégrité des entreprises dans l'intérêt de la société et du développement. 》
La Direction des affaires financières et des entreprises (DAF) aide les gouvernements à améliorer les politiques nationales et mondiales concernant les entreprises et les marchés. Elle travaille sur la lutte contre la corruption, le gouvernement d'entreprise, le droit et la politique de la concurrence, la gestion de la dette, l'investissement, les marchés de capitaux, les assurances, les pensions privées et le développement du secteur privé.

Au moyen d'instruments comme la Convention sur la lutte contre la corruption, la Déclaration sur l'investissement et les Principes de gouvernement d'entreprise, DAF contribue à assurer équité et prévisibilité pour les entreprises. L'adhésion de la Fédération de Russie et de la Colombie à la Convention anti-corruption a porté à 40 le nombre de signataires. DAF continue de collaborer avec des partenaires clés comme la République populaire de Chine et l'Indonésie pour élaborer des législations contre la corruption. La Tunisie est devenue le $44^{\mathrm{e}}$ État à adhérer à la Déclaration sur l'investissement et aux Principes directeurs pour les entreprises multinationales. L'Inde s'est fondée sur les Principes de gouvernement d'entreprise pour réformer son cadre de gouvernement d'entreprise. Le Guide sur le devoir de diligence pour l'achat de minerais a été augmenté d'une section sur l'or. L'OCDE a apporté un soutien et des orientations spécifiques aux pays membres particulièrement affectés par la crise économique, par exemple sur la politique de la concurrence.

DAF appuie la mise en œuvre de la Stratégie de l'OCDE pour le développement par son travail sur l'égalité hommes-femmes, l'autonomisation des femmes et la mobilisation des ressources intérieures. Parmi les treize " Propositions d'action " mises en avant dans la Stratégie pour le développement figurent la révision du Cadre d'action pour l'investissement, utilisé récemment en Malaisie et en Tanzanie, et la facilitation des investissements à long terme en infrastructures dans les pays en développement.

En 2012, les ministres des Finances et les gouverneurs de banques centrales du G20 ont adopté le Cadre méthodologique G20/OCDE pour l'évaluation des risques de catastrophes et leur financement. L'OCDE a présenté les Principes de haut niveau de l'OCDE/INFE sur les stratégies nationales d'éducation financière au Sommet du G20 à Los Cabos en juin 2012. Les travaux sur la protection financière des consommateurs se fondent désormais sur ces Principes. Établi en Afrique du Sud, le Centre pour la gestion de la dette publique et les marchés obligataires en Afrique, géré par l'OCDE et les autorités sud-africaines, permet de réaliser d'importants travaux d'analyse et de renforcer les capacités des marchés obligataires en monnaie locale. Les économies du G20 sont résolument attachées à la liberté des échanges et des investissements, et déterminées à résister au protectionnisme. Depuis 2009, à la demande du G20, l'OCDE, l'OMC et la CNUCED suivent l'évolution des politiques publiques et publient les résultats de ces travaux de surveillance tous les six mois. 


\section{Activités principales}

- Convention sur la lutte contre la corruption : www.oecd.org/fr/daf/anti-corruption

- Lutte contre les soumissions concertées dans les marchés publics : www.oecd.org/daf/competition/fightingbidrigginginpublicprocurement.htm

- Manuel pour l'évaluation d'impact sur la concurrence : www.oecd.org/daf/competition/competitionassessmenttoolkit.htm

- Guide de l'OCDE sur le devoir de diligence pour des chaînes d'approvisionnement responsables en minerais provenant de zones de conflit ou à haut risque : www.oecd.org/fr/daf/inv/mne/mining.htm

- Protection financière des consommateurs : www.oecd.org/fr/finances/financialconsumerprotection.htm

- Marchés financiers : www.oecd.org/fr/daf/fin

- Liberté d'investissement : www.oecd.org/fr/daf/inv/politiques-investissement/foi.htm

- Principes directeurs de l'OCDE à l'intention des entreprises multinationales : www.oecd.org/fr/daf/inv/mne

- Investisseurs institutionnels et investissements à long terme : www.oecd.org/finance/lti

- Assurance : www.oecd.org/fr/daf/fin/assurances

- Portail international sur l'éducation financière : www.financial-education.org

- Principes de gouvernement d'entreprise de l'OCDE : www.oecd.org/daf/affairesdentreprises/principes/texte

- Pensions privées : www.oecd.org/fr/daf/fin/pensions-privees

- Dette souveraine et stabilité financière : www.oecd.org/finance/sovereigndebt

- Les entreprises publiques dans l'économie de marché : www.oecd_org/fr/daf/ae/soemarket

- Gestion de la dette publique : www.oecd.org/fr/daf/fin/dette-publique

\section{Publications principales}

- Rapport annuel sur les Principes directeurs de l'OCDE à l'intention des entreprises multinationales 2012

- Competitive Neutrality: Maintaining a Level Playing Field Between Public and Private Business

- Corporate Governance: Value Creation and Growth

- Guide OCDE sur le devoir de diligence pour des chaînes d'approvisionnement responsables en minerais provenant de zones de conflit ou à haut risque ( $2^{\mathrm{e}}$ édition intégrant un supplément sur l'or)

- Identification et quantification des profits de la corruption - Une analyse OCDE-StAR

- Examens de l'OCDE des politiques de l'investissement : Malaisie, Tunisie, Colombie

- OECD Pensions Outlook 2012

- OECD Sovereign Borrowing Outlook

- Typology on Mutual Legal Assistance in Foreign Bribery Cases (en anglais uniquement)

\section{Manifestations principales}

- Séminaire de haut niveau : "La gestion et la libération des mouvements de capitaux - le rôle de la coopération internationale ", Paris, 9 octobre 2012

- Table ronde OCDE-Asie sur le gouvernement d'entreprise, Tokyo, 24-25 octobre 2012

- Conférence sur l'éducation financière organisée conjointement par la Colombie, l'OCDE et la Banque mondiale : «Progrès des politiques et pratiques mondiales et expériences de l'Amérique latine ", Carthagène, 31 octobre-1 ${ }^{\text {er }}$ novembre 2012

- Amérique latine et Caraïbes : Conférence sur les chaînes de valeurs internationales, San José, 31 octobre-1 ${ }^{\text {er }}$ novembre 2012

- Réunion du Groupe eurasien sur le gouvernement d'entreprise pour le développement des marchés de capitaux, Istanbul, 13 décembre 2012

- $12^{\mathrm{e}}$ Forum mondial sur la concurrence, Paris, 28 février-1 ${ }^{\mathrm{er}}$ mars 2013

- Conférence régionale d'Amérique latine sur la responsabilité du secteur privé dans la lutte contre la corruption, Bogota, 7-8 mars 2013

- Troisième conférence annuelle de haut niveau des gouvernements et entreprises du G20 sur la lutte contre la corruption, organisée conjointement par la Présidence russe du G20 et l'OCDE, avec le soutien de l'ONUDC, Paris, 25-26 avril 2013 


\section{Direction de la gouvernance publique et du développement territorial}

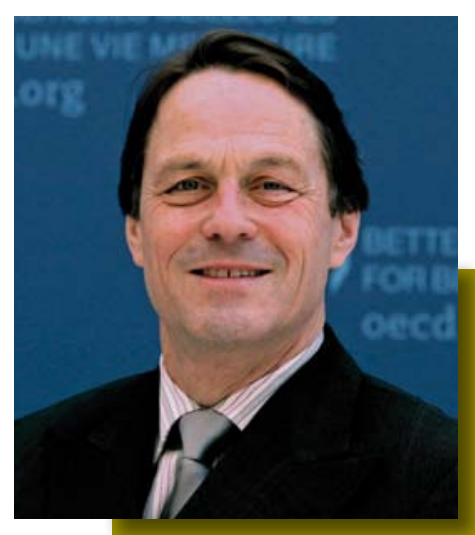

\section{Rolf Alter}

Directeur

Courriel : gov.contact@oecd.org

\section{« L'efficacité des stratégies}

pour créer des emplois et

stimuler la croissance dépend

de l'aptitude des gouvernements

à engendrer un cercle vertueux

de participation citoyenne, de

confiance et de mise en œuvre

effective des politiques. $\gg$
Les incertitudes économiques persistantes pèsent sur les rapports entre gouvernements et citoyens. Les gouvernements peinent à formuler un scénario clair de reprise. Les coupes sans précédent dans les dépenses publiques et le flou entourant les coûts et les avantages des réformes structurelles mènent les citoyens à s'interroger sur l'aptitude des gouvernements à favoriser les emplois et la croissance. L'OCDE offre des sources uniques de données et de vastes travaux d'analyse des politiques sur les manières de construire un secteur public innovant et axé sur les résultats ; de renforcer la gouvernance publique ; et de mener des politiques répondant aux défis divers et perturbants d'aujourd'hui, sur la base d'une plus grande confiance entre citoyens et gouvernements.

En 2012, les grands axes d'action de la Direction ont été des travaux cruciaux sur le thème de l'intégrité ; la publication d'examens sur la gouvernance publique de la France, de la Pologne et de la Slovénie ; la préparation de la phase opérationnelle de l'Observatoire de l'OCDE sur l'innovation dans le secteur public; la deuxième édition de Redresser les finances publiques; et des travaux novateurs d'analyse du développement régional, avec la publication de Redefining Urban: A New Way to Measure Metropolitan Areas and Promoting Growth in All Regions.

Entérinés par le Conseil de l'OCDE, les Principes de l'OCDE applicables à la gouvernance publique des partenariats public-privé viennent, avec les Principes pour une politique et une gouvernance réglementaires, enrichir la gamme des instruments au service d'un secteur public efficace. De nouveaux réseaux de dialogue ont été institués dans des domaines novateurs comme la gouvernance de l'eau, la budgétisation des dépenses de santé et la gestion des risques.

Les pays non membres manifestent un vif intérêt pour les travaux de l'OCDE en matière de gouvernance, qui constituent un pilier important de la Stratégie de l'OCDE pour le développement. Des réseaux régionaux favorisent le dialogue en Asie, en Europe orientale, en Amérique latine, au Moyen-Orient et en Afrique du Nord.

L'année 2013 sera marquée par d'importantes contributions sur le thème de la gouvernance dans le cadre de l'initiative Nouvelles approches face aux défis économiques (NAEC) et par une nouvelle édition du Panorama des administrations publiques, avec des données inédites sur l'administration ouverte et sur la confiance dans les institutions. Une nouvelle édition des Perspectives régionales sera lancée lors de la réunion ministérielle sur les régions et les villes à Marseille en décembre. 


\section{Activités principales}

- Budgétisation et dépenses publiques : www.oecd.org/gov/budget-fr

- Lutte contre la corruption dans le secteur public : www.oecd.org/gov/ethique

- Panorama des administrations publiques : www.oecd.org/gov/indicateurs/panoramaapu

- Emploi et gestion publics : www.oecd.org/gov/grh

- Réforme de la gouvernance publique au Moyen-Orient et en Afrique du Nord : www.oecd.org/mena/governance

- Innovation dans le secteur public et administration électronique : www.oecd.org/gov/adminelectronique

- Statistiques et indicateurs régionaux : www.oecd.org/gov/regional/statistiquesindicateurs

- Développement urbain, rural et régional : www.oecd.org/gov/regional-fr

- Politique réglementaire : www.oecd.org/gov/reformereg

- SIGMA (Soutien à l'amélioration des institutions publiques et des systèmes de gestion) : www.oecd.org/gov/sigma

- Gestion des risques: www.oecd.org/gov/risques

\section{Publications principales}

- Redresser les finances publiques : mise à jour 2012

- Public Sector Compensation in Times of Austerity

- Value for Money in Government: Australia

- OECD Reviews of Regulatory Policy: Indonesia - Strengthening Coordination and Connecting Markets

- Measuring Regulatory Performance: A Practitioner's Guide to Perception Surveys

- Examens de l'OCDE sur la gouvernance publique : France - Une perspective internationale sur la révision générale des politiques publiques

- Compact City Policies: A Comparative Assessment

- Meeting the Water Reform Challenge

- Redefining "Urban": A New Way to Measure Metropolitan Areas

- Promoting Growth in All Regions

Périodiques :

- Revue de l'OCDE sur la gestion budgétaire

- Examens de l'OCDE sur la gouvernance publique

- Examens de l'OCDE de la réforme de la réglementation

- Examens territoriaux de l'OCDE

\section{Manifestations principales}

- 4 e Table ronde de l'OCDE des maires et des ministres : Mobiliser l'investissement au service de la durabilité urbaine, de la création d'emplois et d'une croissance résiliente, Chicago, 8 mars 2012

- Réunion annuelle des Hauts responsables du budget, Reykjavik, 7 et 8 juin 2012

- Réunion du Réseau des Hauts responsables des centres de gouvernement, Londres, 23 octobre 2012

- Premier Forum mondial sur la gouvernance publique, Paris, 21 novembre 2012

- Forum de haut niveau sur les risques, Paris, décembre 2012 


\section{Direction de la science, de la technologie et de l'industrie}

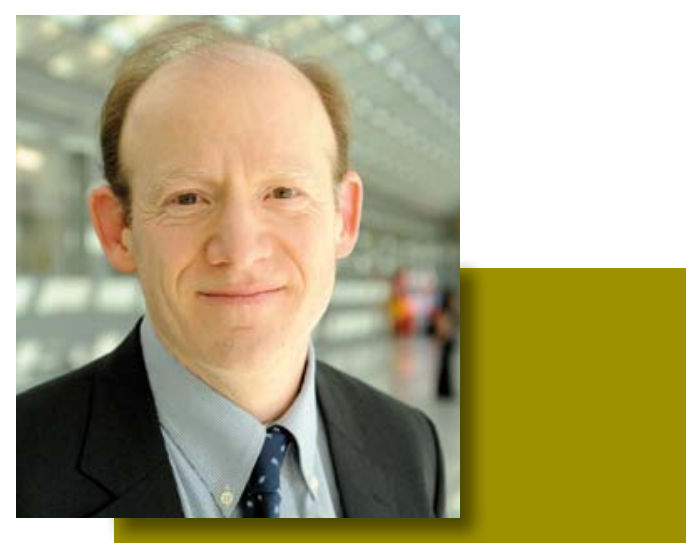

Andrew Wyckoff

Directeur

Courriel : sti.contact@oecd.org

« Pour promouvoir une

croissance durable et les emplois

de demain, les pouvoirs publics

doivent faciliter l'investissement

des entreprises dans les actifs

intellectuels. L'investissement

dans des domaines comme

la recherche, la conception,

les logiciels, les grandes bases

de données, les réseaux et

les organisations intelligentes

stimuleront la productivité,

renforceront la compétitivité et

libéreront de nouvelles sources

de croissance. $\gg$
Les investissements sont de plus en plus souvent déterminés par les actifs intellectuels et nous allons étudier plus en détail le potentiel d'actifs tels que les données massives, le design, et la propriété intellectuelle au service d'une croissance sociale et économique durable.

À mesure que l'économie mondiale continue de s'adapter à la mondialisation, les processus et l'implantation des activités de production se transforment, ainsi que les facteurs qui conduisent à la création de valeur. Le projet conjoint avec la Direction des statistiques (STD) et la Direction des échanges et de l'agriculture (TAD) sur les échanges en valeur ajoutée (ÉVA) a débouché sur des indicateurs innovants permettant d'améliorer la cartographie des réseaux de production mondiaux et d'analyser la chaîne de la valeur ajoutée créée à chaque stade. Les prochains travaux examineront plus en détail le rôle croissant de la connaissance dans les chaînes de valeur mondiales pour la croissance, la création d'emplois et l'évolution future de l'activité manufacturière.

La science, la technologie et l'innovation peuvent aussi contribuer à répondre aux défis planétaires comme le changement climatique, le développement durable, le vieillissement démographique et les inégalités. Ainsi, les progrès de la nanotechnologie et de la biomédecine pourraient conduire à des gains significatifs dans le secteur de la santé, tandis que les technologies de l'information et des communications peuvent aider les personnes âgées à garder plus longtemps leur autonomie. Notre projet sur l'innovation inclusive, en collaboration avec EDU, GOV et $\mathrm{DCD}$, contribuera à la Stratégie de l'OCDE pour le développement.

Nos travaux sur l'examen du cadre de mesure de l'innovation et l'élaboration d'une infrastructure de données permettant de retracer les flux mondiaux de connaissance, ainsi que l'utilisation de solides éléments d'observation pour l'évaluation des politiques, contribueront à une meilleure compréhension des sources de croissance. Enfin, nous espérons accroître la pertinence de nos statistiques et analyses des politiques en dispensant des conseils mieux adaptés à chaque pays par le biais, par exemple, d'outils interactifs sur le web, comme la prochaine édition électronique des Perspectives STI et la plateforme sur les politiques d'innovation.

La Direction de la science, de la technologie et de l'industrie de l'OCDE élabore, à partir de données factuelles, des conseils sur les politiques à suivre concernant la contribution de la science, de la technologie et de l'industrie au bien-être et à la croissance économique. 


\section{Activités principales}

- Innovation : www.oecd.org/fr/innovation

- Économie de l'Internet : www.oecd.org/fr/internet

- Haut débit et télécoms : www.oecd.org/fr/sti/hautdebit

- Science et technologie : www.oecd.org/fr/science

- Biotechnologie : www.oecd.org/fr/sti/biotech

- Industrie et globalisation : www.oecd.org/fr/sti/ind

- Politique des consommateurs : www.oecd.org/fr/sti/consommateurs

\section{Publications principales}

- Encourager l'investissement dans le capital intellectuel, la croissance et l'innovation

- Perspectives des communications de l'OCDE 2013

- Interconnected Economies: Benefiting from Global Value Chains

- Principaux indicateurs de la science et de la technologie

- Science, technologie et industrie : Tableau de bord de l'OCDE 2013

- Space Technologies and Food Security

- Science, technologie et industrie : Perspectives de l'OCDE 2012

- Perspectives de l'économie Internet de l'OCDE 2012

- Examens de l'OCDE des politiques d'innovation : Asie du Sud-Est, Slovénie, Suède, Vietnam

- Les besoins d'infrastructures transcontinentales à l'horizon 2030

\section{Manifestations principales}

- Forum mondial sur l'économie du savoir, Séoul, 18-19 septembre 2012

- Forum mondial sur la biotechnologie, Paris, 12 novembre 2012

- Statistiques de brevets à l'intention des décideurs politiques, Paris, 28-29 novembre 2012

- Conférence finale : Maximiser les retombées des actifs intellectuels, Paris, 13-14 février 2013

- Forum mondial sur l'économie du savoir, Istanbul, 22-23 octobre 2013

- Statistiques de brevets à l'intention des décideurs politiques, Paris, 12-13 novembre 2012 


\section{Direction des statistiques}

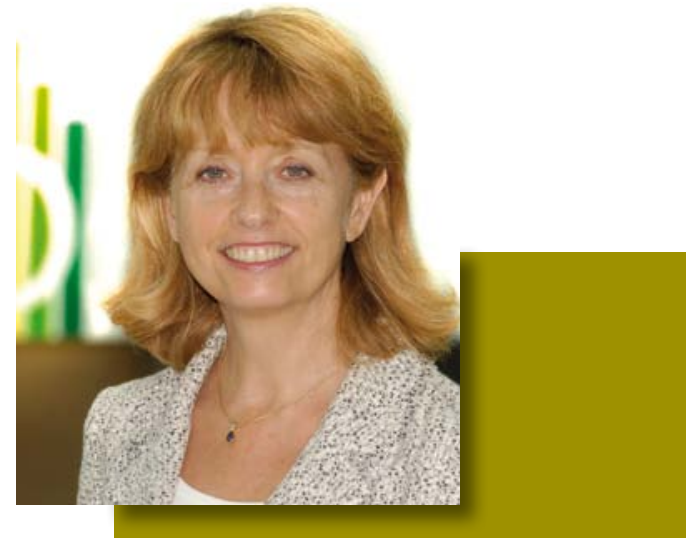

Martine Durand

Directrice et Chef statisticienne Courriel : stat.contact@oecd.org

« Nous devons nous adapter

à la réalité changeante et de

plus en plus complexe des

échanges de biens et services.

L'action publique, et notamment

les négociations commerciales,

doit reposer sur des données

probantes. C'est pourquoi nous

travaillons en collaboration

avec d'autres Directions et

l'Organisation mondiale du

commerce pour élaborer des

mesures qui apporteront un

nouvel éclairage sur la valeur

ajoutée effective des échanges

et leur impact sur les emplois,

les salaires et les bénéfices. 》)
La Direction des statistiques (STD), la Direction de la science de la technologie et de l'industrie (STI), et la Direction des échanges, en collaboration avec l'Organisation mondiale du commerce (OMC), ont récemment lancé une base de données d'indicateurs des Échanges en valeur ajoutée, et visent à intégrer la production de ces indicateurs dans le système statistique international. Également en collaboration avec STI, STD a élaboré le Compendium de l'OCDE sur les indicateurs de productivité 2012, qui constitue une réponse opportune au besoin croissant de conseils sur les politiques concernant les questions liées à la productivité et à la compétitivité internationale.

Face aux inégalités persistantes entre hommes et femmes, STD a récemment lancé, en collaboration avec la Direction de l'emploi, du travail et des affaires sociales et la Direction de l'éducation et des compétences, le portail de données hommes-femmes de l'OCDE, qui regroupe une sélection d'indicateurs illustrant les inégalités entre les sexes en matière d'éducation, d'emploi et d'entrepreneuriat.

Les travaux sur la mesure du bien-être se poursuivent. Les conclusions de Comment va la vie?, rapport offrant une vision globale du bien-être dans les pays de l'OCDE et d'autres grandes économies, ont constitué la base des débats du $4^{e}$ Forum mondial de l'OCDE intitulé "Statistiques, connaissances et politiques : Mesurer le bien-être pour les politiques de développement ", à New Delhi en octobre 2012. En collaboration avec la Direction de l'environnement, STD a poursuivi les travaux du rapport sur les indicateurs de croissance verte, présenté lors de la Réunion du Conseil au niveau des ministres de 2011, dans le cadre de la Stratégie de l'OCDE pour une croissance verte. Ces travaux ont été axés sur l'élaboration d'indicateurs phares, de contributions à la Plateforme de connaissances sur la croissance verte, et la rédaction d'applications et d'extensions des nouvelles normes internationales de comptabilité environnementale et économique, destinées à les compléter. La Direction des statistiques a également lancé le processus qui permettra à terme de réunir des tableaux de base fondés sur ces nouvelles normes internationales.

La Direction des statistiques de l'OCDE recueille et analyse des statistiques économiques et sociales émanant des pays membres et partenaires de l'Organisation. Son objectif est de diffuser des données aussi comparables que possible au niveau international. La Direction est responsable de la coordination de l'ensemble des activités statistiques de l'OCDE et du développement de son système d'information statistique. Elle œuvre à la diffusion des statistiques clés de l'OCDE au moyen d'instruments comme le Panorama des statistiques de l'OCDE, des graphiques dynamiques et des outils en ligne innovants. 


\section{Activités principales}

- L'initiative du vivre mieux de l'OCDE :

www.oecd.org/fr/statistiques/linitiativeduvivremieuxmesurerlebien-etreetleprogres.htm

- Indicateurs composites avancés et enquêtes de conjoncture : www.oecd.org/fr/std/clits

- Indicateurs de l'entrepreneuriat et statistiques sur les entreprises : www.oecd.org/fr/std/stats-entreprises

- Statistiques financières : www.oecd.org/fr/std/stats-fin

- Égalité hommes-femmes : www.oecd.org/fr/parite

- Stratégie pour une croissance verte : www.oecd.org/fr/croissanceverte

- Échanges internationaux et balance des paiements : www.oecd.org/fr/std/stats-echanges

- Statistiques sur le marché du travail : www.oecd.org/fr/std/stats-travail

- Mesurer le bien-être et le progrès : www.oecd.org/mesurerleprogres

- Mesurer les Échanges en valeur ajoutée : www.oecd.org/fr/industrie/ind/mesurerlecommerceenvaleurajouteeuneinitiativeconjointedelocdeetdelomc.htm

- Comptes nationaux : www.oecd.org/fr/std/cn

- Prix et parités de pouvoir d'achat : www.oecd.org/fr/std/prix-ppa

- Statistiques sur la productivité : www.oecd.org/fr/std/stats-productivite

\section{Publications principales}

- Comment va la vie? - Mesurer le bien-être

- Panorama de l'entrepreneuriat 2013

- Panorama des comptes nationaux 2013

- OECD Compendium of Productivity Indicators 2012 (en anglais uniquement)

- Panorama des statistiques de l'OCDE 2013 - Économie, environnement et société

\section{Manifestations principales}

- Conférence européenne : "Mesurer le bien-être et favoriser le progrès des sociétés », Paris, 26-28 juin 2012

- $4^{\mathrm{e}}$ Forum mondial de l'OCDE : «Statistiques, connaissances et politiques : Mesurer le bien-être pour les politiques de développement », New Delhi, 16-19 octobre 2012

- Conférence conjointe OCDE-Universités : "Politiques économiques pour un monde meilleur », Paris, 3-5 juillet 2013 


\section{Centre de politique et d'administration fiscales}

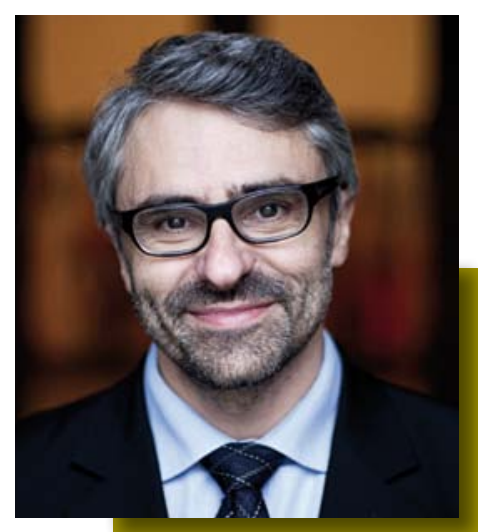

Pascal Saint-Amans Directeur

Courriel : ctp.contact@oecd.org

« La crise majeure que nous traversons se répercute aussi dans le domaine fiscal, car elle fait peser une charge supplémentaire sur les citoyens presque partout et ébranle la confiance dans le système fiscal dans de nombreux pays. Rétablir cette confiance tout en favorisant une conception de l'impôt favorable à la croissance est au cœur de nos efforts. Pour répondre à l'appel lancé par le G20, nous mettrons l'accent sur la lutte contre l'érosion de la base d'imposition et le transfert de bénéfices, qui aboutissent à une double exonération involontaire. Un Plan d'action sera élaboré au cours du premier semestre en vue de restaurer la confiance dans l'équité de nos systèmes fiscaux. 》)
Le Centre de politique et d'administration fiscales (CTPA) est le pivot des travaux de l'OCDE dans le domaine fiscal. Le Centre fournit une expertise et un soutien techniques au Comité des affaires fiscales. Son activité couvre les questions fiscales nationales et internationales, les impôts directs et indirects ainsi que la politique et l'administration fiscales. Les travaux du CTPA bénéficient d'importantes contributions d'économies membres et non membres de l'OCDE, ainsi que de l'éclairage des représentants des entreprises et de la société civile.

Supprimer la double imposition afin de faciliter l'investissement international et renforcer la coopération fiscale entre administrations restent les missions essentielles du CTPA. Nous avons activement aidé les pays membres à améliorer l'échange de renseignements et à accroître la transparence. Le Forum mondial a commencé à examiner l'efficacité des échanges de renseignements et nous avons soutenu la mise en œuvre de léchange automatique pour les pays intéressés. Nous avons également lancé d'importants travaux afin de lutter contre l'érosion de la base d'imposition et le transfert de bénéfices, qui aboutissent à une double exonération involontaire. Ces efforts sont essentiels pour garantir l'équité de nos systèmes fiscaux et sont fortement encouragés par les ministres des Finances du G20, qui nous ont demandé d'élaborer un Plan d'action d'ici juin 2013. Le CTPA a également affiné son analyse des politiques fiscales en vue de promouvoir des systèmes fiscaux efficients et respectueux de l'environnement : ainsi, pour la première fois, nous avons mesuré les taux effectifs d'imposition de la consommation d'énergie, et les données ainsi obtenues seront utiles aux responsables de la politique publique. Enfin, nous avons renforcé nos travaux sur la fiscalité et le développement, qui englobent les prix de transfert, les incitations fiscales ainsi que le lancement de l'initiative «Inspecteurs des impôts sans frontières ». 


\section{Activités principales}

- Érosion de la base d'imposition et transfert de bénéfices : www.oecd.org/fr/fiscalite/beps-fr.htm

- Base de données fiscales de l'OCDE : www.oecd.org/ctp/taxdatabase

- Analyse des politiques fiscales : www.oecd.org/ctp/apf

- Conventions fiscales : www.oecd.org/ctp/cf

- Prix de transfert : www.oecd.org/ctp/pt

- Convention concernant l'assistance administrative mutuelle en matière fiscale : www.oecd.org/ctp/eoi/mutual

- Impôts sur la consommation : www.oecd.org/ctp/ic

- Échange de renseignements : www.oecd.org/fr/ctp/echange-de-renseignements-fiscaux

- Administration fiscale : www.oecd.org/tax/fta

- Fiscalité et délits : www.oecd.org/ctp/delitsfiscaux

- Relations internationales et fiscalité : www.oecd.org/fr/fiscalite/fiscalite-internationale

- Initiative fiscale pays d'Amérique latine et des Caraïbes : www.oecd.org/tax/lacfiscal

\section{Publications principales}

- Automatic Exchange of Information: What It Is, How It Works, Benefits, What Remains To Be Done

- Garantir la confidentialité : Le guide de l'OCDE sur la protection des échanges de renseignements à des fins fiscales

- Statistiques des recettes publiques 1965-2011, édition 2012

- Consumption Tax Trends 2012

- Revenue Statistics in Latin America, 1990-2010

- Taxing Energy Use: A Graphical Analysis

- Lutter contre l'érosion de la base d'imposition et le transfert de bénéfices

- Electronic Sales Suppression: A Threat to Tax Revenues

- Planification fiscale agressive fondée sur des opérations de couverture après impôt

- Comparative Information Series 2013

- Les impôts sur les salaires 2012

\section{Manifestations principales}

- Conférence fiscale internationale OCDE-USCIB, Washington, DC, 4-5 juin 2012

- $2^{e}$ Forum mondial Fiscalité et Délinquance, Rome, 14-15 juin 2012

- $2^{\mathrm{e}}$ réunion du Forum Amérique latine - Caraïbes sur la politique fiscale, Bogota, 12-13 juillet 2012

- Forum mondial sur les conventions fiscales et les prix de transfert : $17^{e}$ réunion annuelle internationale sur les conventions fiscales, Paris, 12-13 septembre 2012

- Forum mondial sur la transparence et l'échange de renseignements, Le Cap, 26-27 octobre 2012

- Forum mondial sur la TVA, Paris, 7-8 novembre 2012

- Table ronde OCDE/FMI : « La politique fiscale pour une croissance plus verte », Paris, 24 janvier 2013

- $2^{\mathrm{e}}$ réunion annuelle du Forum mondial sur les prix de transfert, Paris, 6-7 mars 2013

- Lutter contre la planification fiscale agressive, les délits fiscaux et la fraude fiscale par une meilleure coopération internationale, Inde, 16-17 avril 2013

- $8^{e}$ Forum sur l'administration de l'impôt, Moscou, 16-17 mai 2013

- Conférence fiscale internationale OCDE-USCIB, Washington, DC, 3-4 juin 2013 


\section{Direction des échanges et de l'agriculture}

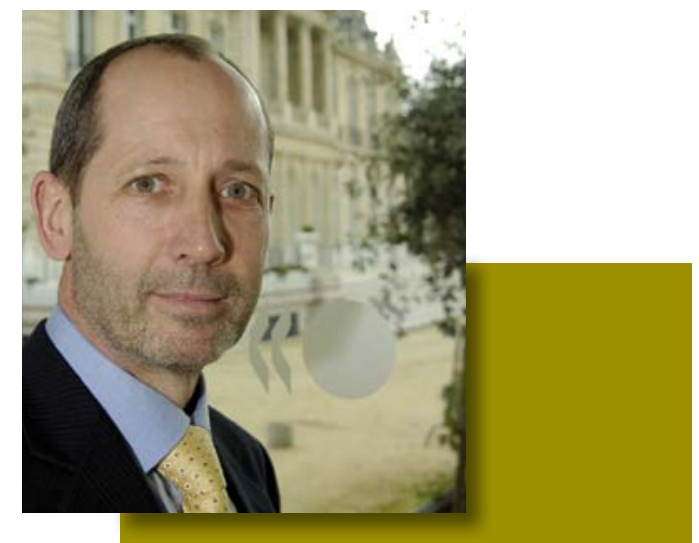

Ken Ash

Directeur

Courriel : tad.contact@oecd.org

« L'émergence de chaînes de valeur mondiales et de nouvelles mesures du revenu généré par les échanges confirment le bien-fondé de la suppression des obstacles aux frontières et d'ouvrir davantage les marchés, en particulier pour les échanges de services. L'ouverture des échanges requiert aussi des investissements publics et privés complémentaires - en ressources humaines, en compétences et en innovation. Cela vaut également pour des secteurs traditionnellement sensibles comme l'agriculture, où des subventions souvent inefficaces ne sont guère justifiées, compte tenu en particulier de la vigueur du marché. \$)
Les travaux de l'OCDE relatifs aux échanges visent à éclairer la relation entre l'ouverture commerciale et une croissance inclusive. Les travaux sur les échanges, la croissance et l'emploi, et le rôle de politiques complémentaires, se poursuivent. La nouvelle base de données sur les Échanges en valeur ajoutée est un outil innovant pour mieux comprendre la structure économiques sous-jacente et les bénéficiaires des échanges. L'OCDE a aussi élaboré une série d'indicateurs de facilitation des échanges identifiant des domaines d'action concrets.

La couverture géographique et sectorielle des travaux sur les services s'étend. Une base de données sur les réglementations et des indices de restrictivité des échanges de services sont construits pour 40 pays et pour tous les principaux secteurs de services, pour être publiés à la mi-2014. Les travaux de l'OCDE sur les restrictions commerciales dans des secteurs sensibles comme les matières premières et l'agriculture complètent ceux réalisés auparavant sur les restrictions à l'importation et contribuent à identifier les effets dommageables de ces mesures ainsi que les autres outils plus efficaces pour atteindre les résultats souhaités.

L'OCDE contribue aux efforts de ses membres pour réformer leur agriculture en fournissant des analyses approfondies sur l'impact des mesures appliquées, la gestion des risques, les politiques en matière d'échanges agricoles, les performances environnementales et les systèmes d'innovation. L'édition 2012 des Perspectives agricoles à moyen terme de l'OCDE et de la FAO contient une étude spéciale sur les politiques relatives aux biocarburants, et l'édition 2013 offrira une étude sur la République populaire de Chine. Le rapport de l'OCDE intitulé Politiques agricoles : Suivi et évaluation présente les seules mesures comparables à l'échelle internationale des aides agricoles dans les pays de l'OCDE. L'édition 2013 couvrira également le Brésil, la République populaire de Chine, la Fédération de Russie, l'Afrique du Sud, l'Ukraine, l'Indonésie et le Kazakhstan. En 2013, l'OCDE publiera un rapport de synthèse complet sur la sécurité alimentaire dans le monde, énonçant les principales conclusions et recommandations des travaux réalisés ces dernières années, notamment pour le G20. L'OCDE travaille aussi sur un large éventail de questions relatives aux politiques de la pêche et de l'aquaculture et a publié en 2012 un important rapport intitulé Redressement des pêches : Le cap à suiure.

La Direction des échanges et de l'agriculture (TAD) soutient la mise en place d'un système commercial multilatéral fort et encadré. Elle aide les gouvernements à concevoir et mettre en œuvre des politiques d'échanges, d'agriculture et de pêche propices à une croissance durable et créatrice d'emplois. Elle gère également les programmes relatifs aux crédits à l'exportation bénéficiant d'un soutien public, et ceux traitant des normes applicables aux produits agricoles qui facilitent les échanges et la recherche collaborative sur les systèmes agricoles durables. 


\section{Activités principales}

- Politiques agricoles : www.oecd.org/agriculture/politiques

- Échanges agricoles : www.oecd.org/agriculture/echanges

- Bénéfices de la libéralisation des échanges : www.oecd.org/tad/beneficelib

- Environnement et échanges : www.oecd.org/echanges/env

- Crédits à l'exportation : www.oecd.org/echanges/xcred/

- Pêcheries : www.oecd.org/pecheries

- Échanges et développement : www.oecd.org/echanges/dev

- Échanges et emploi : www.oecd.org/echanges/emploi

- Facilitation des échanges : www.oecd.org/echanges/facilitation

- Échanges de services : www.oecd.org/echanges/services

- Normes pour les semences, tracteurs, matériel forestier, fruits et légumes : www.oecd.org/agriculture/code-fr

- Agriculture durable : www.oecd.org/agriculture/durable

\section{Publications principales}

- Perspectives agricoles de l'OCDE et de la FAO 2013-2022

- Politiques agricoles: suivi et évaluation - Pays de l'OCDE et économies émergentes

- Compendium des Indicateurs Agro-Environnementaux de l'OCDE

- Inventory of Estimated Budgetary Support and Tax Expenditures for Fossil Fuels 2013

- Agricultural Policies for Poverty Reduction

- Livestock Diseases: Prevention, Control and Compensation Schemes

- Redressement des pêches : Le cap à suiure

- Review of Agricultural Policies: Indonesia 2012

- OECD Review of Agricultural Policies: Kazakhstan 2013

- Qualité de l'eau et agriculture : Un déf pour les politiques publiques

\section{Manifestations principales}

- Forum mondial sur les échanges - Échanges de services : enjeux et conséquences, Paris, 8 novembre 2012

- Forum mondial sur l'agriculture - La cohérence des politiques au service de la sécurité alimentaire dans les pays en développement, Paris, 26 novembre 2012

- Lancement officiel de la base de données sur les Échanges en valeur ajoutée, Paris, 16 janvier 2013

- International Business Dialogue: Barriers to 21 ${ }^{\text {st }}$ Century Commerce, Paris, 4-5 mars 2013

- Réunion sur le Système d'information sur les marchés agricoles (AMIS), Paris, 22-24 avril 2013

- G20-OECD GVC-TiVA Stocktaking Seminar, Paris, 29 mai 2013 


\section{Secrétariat des relations mondiales}

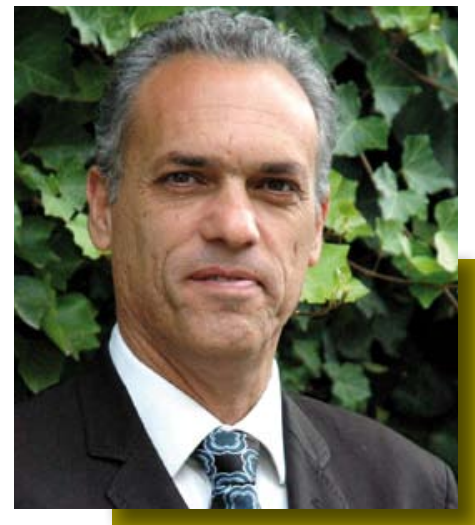

Marcos Bonturi

Directeur

Courriel : grs.contact@oecd.org

« L'OCDE favorise le partage

de connaissances entre des

pays qui se trouvent à différents

stades de développement, pour

qu'ils puissent parvenir à une

convergence de vues sur les

enjeux communs. Elle favorise

le dialogue sur les politiques

afin d'assurer un développement

économique inclusif et durable.

La participation des pays

partenaires aux travaux de

l'OCDE apporte des perspectives, des idées et des expériences

nouvelles qui viennent renforcer

la pertinence des discussions sur

les politiques publiques. $\boldsymbol{M}$
Le Secrétariat des relations mondiales conseille le Secrétaire général sur les orientations stratégiques de l'Organisation à l'égard de ses partenaires. Il facilite l'engagement des différentes communautés de l'OCDE auprès des pays partenaires et contribue à l'élaboration des programmes de travail avec les partenaires clés et les autres pays et régions qui présentent une importance stratégique.

L'OCDE offre un réseau mondial qui aide les membres et les pays partenaires à parvenir à une convergence de vues sur les difficultés auxquelles ils doivent tous faire face et les moyens d'y remédier. En s'appuyant sur les recommandations de l'Organisation, fondées sur des données concrètes, ils mettent en commun leurs connaissances et leur savoir-faire à l'appui des réformes politiques. Cette démarche garantit à long terme la pertinence générale de l'Organisation.

L'OCDE travaille en collaboration étroite avec ses partenaires clés - le Brésil, la République populaire de Chine, l'Inde, l'Indonésie et l'Afrique du Sud - dans tout l'éventail de ses programmes de travail, y compris à travers ses contributions au G20. Elle cherche à créer des mécanismes permettant de les associer plus étroitement à la définition des priorités des travaux communs. Il est important que leurs intérêts et leurs points de vue contribuent à la mise en forme de ses activités.

L'Organisation renforce aussi son association avec d'autres économies émergentes. L'objectif est de soutenir leur développement et leur processus de réforme en les aidant à tirer parti de ses connaissances en matière de politiques et de normes mondiales dans différents domaines thématiques. En outre, un nouvel élan est donné aux programmes et réseaux régionaux de l'OCDE, en particulier en Asie du Sud-Est, que les membres considèrent comme une région d'intérêt stratégique. 


\section{Activités principales}

- Relations mondiales de l'OCDE, partenaires clés, programmes régionaux et forums mondiaux :

www.oecd.org/fr/relationsmondiales/

- Afrique : www.oecd.org/fr/afrique

- Brésil : www.oecd.org/fr/bresil

- Chine : www.oecd.org/fr/chine

- Inde : www.oecd.org/fr/inde

- Indonésie : www.oecd.org/fr/indonesie

- Afrique du Sud : www.oecd.org/fr/afriquedusud

- Amérique latine : www.oecd.org/latinamerica

- Moyen-Orient et Afrique du Nord : www.oecd.org/mena

\section{Publications principales}

- China in Focus, Lessons and Challenges

Brochures OCDE :

- Active with Africa, Special Focus on South Africa

- Active with Brazil, For a Stronger, Cleaner, Fairer Economy

- Active with India, Better Policies for Better Lives

- Active with Latin America and the Caribbean, Better Policies for Better Lives Active with the People's Republic of China, Better Policies for Better Lives

- Active with Southeast Asia: Special Focus on Indonesia 


\section{Relations extérieures et communication}

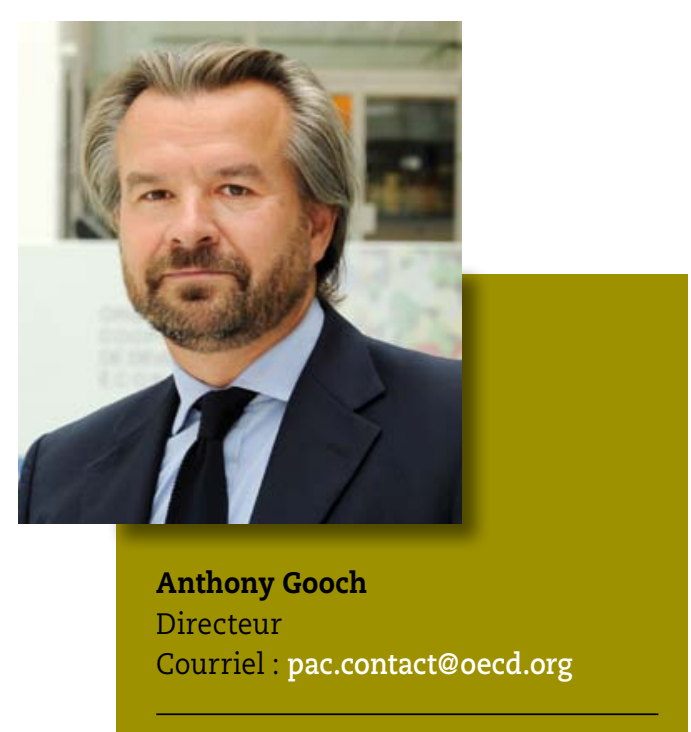

« En temps de crise, le public a besoin d'informations claires et précises pour mieux comprendre les défis auxquels nos sociétés sont confrontées et la manière de les relever. Notre tâche consiste à montrer aux citoyens et aux dirigeants comment les travaux de l'OCDE contribuent à améliorer la cohésion et à rétablir la confiance. Des initiatives comme l'Indicateur du vivre mieux et le Forum de l'OCDE encouragent davantage de gens à participer activement à l'élaboration de politiques meilleures pour une vie meilleure. $\gg$
La Direction des relations extérieures et de la communication (PAC) a continué à valoriser les transformations majeures survenues dans ses sphères de compétence - communication, relations extérieures, diplomatie et diffusion - pour renforcer la capacité de l'OCDE à optimiser les politiques publiques et à améliorer la vie des citoyens des pays membres et du monde entier. Nous avons développé notre rôle de direction et de coordination des campagnes de communication conduites au nom de l'OCDE, rehaussant la valeur mesurable et l'impact de l'Organisation, tout en dynamisant la diffusion et en améliorant le dialogue et la coopération avec les toutes les parties prenantes non-gouvernementales, partout dans le monde.

Des campagnes proactives orchestrées autour des priorités de communication promouvoir une croissance inclusive, restaurer la confiance et favoriser la durabilité - ont permis de positionner l'OCDE au cœur des principaux débats politiques et des grandes questions mondiales, comme la lutte contre les inégalités et la transparence fiscale. PAC a activement promu les travaux novateurs de l'OCDE sur les actifs intellectuels, les chaînes de valeur mondiales et les échanges en valeur ajoutée, tout en continuant à promouvoir les initiatives transversales clés de l'OCDE sur la croissance verte, la parité, l'innovation et le bien-être, et à être pionnier sur le bien-être dans le cadre de l'Initiative du vivre mieux. PAC a joué un rôle clé dans le développement d'un réseau de personnalités et d'entités non-gouvernementales associées au projet Nouvelles approches face aux défis économiques.

L'Indicateur du vivre mieux de l'OCDE, qui promeut le rôle prépondérant de l'Organisation dans le débat mondial sur le bien-être et la qualité de vie, a connu un succès majeur, avec 1,7 million d'utilisateurs dans plus de 180 pays, accroissant considérablement la participation des citoyens et la prise en compte de leur voix par l'OCDE. L'impact du lancement de l'Indicateur en français et en russe laisse augurer un engouement mondial renouvelé pour cette initiative.

L'année dernière, certaines publications et prévisions phares de l'OCDE, comme l'Étude économique de l'UE (+ $170 \%$ ), Regards sur l'éducation (+ $90 \%$ ), les Perspectives économiques de mai 2012 (+ $60 \%$ ) et les Perspectives de l'emploi (+ $50 \%)$, ont vu leur impact médiatique progresser considérablement par rapport aux éditions précédentes.

Nos divers circuits de diffusion ont eu un succès notable : en 2012, la diffusion des contenus de l'OCDE a progressé de plus de $20 \%$ (12 millions de téléchargements, consultations en ligne et publications) ; le nombre de visites de OECD iLibrary a atteint 4 millions $(+40 \%)$; un demi-million de personnes ont téléchargé le Panorama des statistiques de l'OCDE. Le site www.oecd.org a été amélioré, notamment en renouvelant la visualisation de données. Nous avons aussi accru notre présence sur les médias sociaux, avec 70000 abonnés à nos comptes Twitter. 
Au-delà de son rôle de plateforme de connaissances, l'OCDE devient, grâce à PAC, une organisation « à l'écoute ", s'adressant à de nouveaux publics et élargissant ses partenariats avec des fondations et des think tanks internationaux, tels que l'Institut pour une nouvelle pensée économique, l'Observer Research Foundation (Inde) et l'Académie présidentielle russe d'économie nationale et d'administration publique. Elle continue à favoriser le dialogue, avec le Forum de l'OCDE ( $80 \%$ des 2000 participants au Forum 2012 l'ont jugé « excellent » ou « très bon "), l'élargissement du Réseau parlementaire mondial et la multiplication des liens et interactions avec les entreprises (BIAC), les syndicats (TUAC) et les ONG. L'Observateur de l'OCDE et L'Annuel de l'OCDE demeurent des instruments de promotion de débats de haut niveau entre responsables de l'action publique, parties prenantes et experts de l'Organisation.

À l'horizon 2015, l'OCDE vise l'accès gratuit à toutes ses données. Ce projet soulève des défis de communication, technologiques et d'investissement, et son succès se mesurera à l'aune de la progression de la diffusion et de la "démocratisation » des travaux de l'Organisation. Nos divisions Médias, Affaires publiques et Édition, ainsi que les Centres de l'OCDE de Berlin, Mexico, Tokyo et Washington, coordonneront les efforts en ce sens.

\section{Activités principales}

- Centres à l'étranger : www.oecd.org/centres-fr

- Société civile : www.oecd.org/societecivile

- i-Library, diffusion et connaissances : www.oecd-ilibrary.org

- Images et vidéos : www.flickr.com/OECD; www.youtube.com/OECD

- Site Internet : www.ocde.org

- Relations avec les médias : www.oecd.org/media-fr

- Forum de l'OCDE : www.oecdforum.org

- L'Observateur de l'OCDE : www.observateurocde.org

- L'Annuel de l'OCDE : www.oecd.org/annuel

- Relations avec les parlementaires : www.oecd.org/parlementaires

- Édition : www.oecd.org/editions

- Web 2.0: www.facebook.com/TheOECD; www.twitter.com/OCDE_francais

- Le bien-être, « au-delà du PIB » : www.oecdbetterlifeindex.org/fr

\section{Publications principales}

- L'Observateur de l'OCDE, édition spéciale 50e anniversaire : “ Gouvernement et révolution de l'information ", n² 293, T4 2012

- L'Annuel de l'OCDE 2013

- Les Essentiels de l'OCDE : www.oecd.org/insights

- Blogs : OECD Insights http://oecdinsights.org et sur www.oecdbetterlifeindex.org/fr

- Panorama des statistiques de l'OCDE

- Rapport du Secrétaire général aux ministres 2013

- Visualisation de données : Horizon 2060 : Perspectives de croissance économique globale à long terme : meilleure vidéo 2012, avec 40000 vues sur une période de deux mois ; et premier concours de visualisation des données

\section{Manifestations principales}

- Forum de l'OCDE 2012: « Comment passer de l'indignation et l'inégalité à l'inclusion et à l'intégrité », Paris, 22-23 mai 2012

- Allocution du Secrétaire général lors d'une conférence réunissant des responsables de la société civile (Global Perspectives Conference), Berlin, 31 octobre 2012

- Premières Journées parlementaires de l'OCDE, Paris, 11-13 février 2013

- Forum de l'OCDE 2013 : « Emploi, égalité et confiance », Paris, 28-29 mai 2013

- Séminaire parlementaire de haut niveau de l'OCDE, organisé par le Parlement suédois, Stockholm, 17-18 juin 2013 


\section{Direction exécutive}

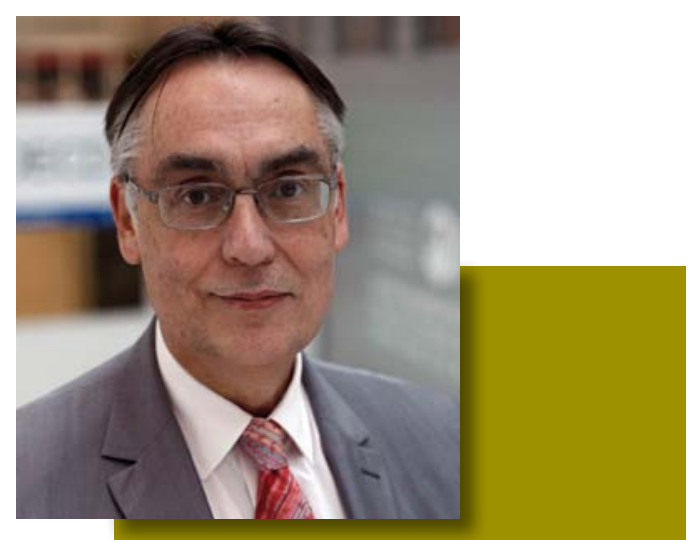

Anthony Rottier

Directeur

Courriel : accountexdinfo@oecd.org

« La Direction exécutive assure

à l'Organisation les moyens dont

elle a besoin pour réaliser ses

objectifs stratégiques, et pour

le faire de façon aussi efficiente

et efficace que possible. En tant

que nouveau Directeur exécutif

depuis la fin 2012, mon objectif

est de de renforcer cette action et

de veiller à ce que les politiques

et pratiques de gestion de l'OCDE

restent à l'avant-garde des

organisations internationales. \$)
La Direction exécutive (EXD) met en place les politiques et les moyens opérationnels nécessaires à l'optimisation des ressources humaines et financières de l'OCDE. Elle contribue aux travaux de l'Organisation en se chargeant des technologies de l'information et de la communication ainsi que des conférences, de la sécurité et des services linguistiques, et elle veille à ce que les locaux et la gestion opérationnelle du site offrent aux délégués, aux visiteurs et aux agents les moyens d'être aussi productifs que possible.

En 2012, EXD a été étroitement associée à l'élaboration et à la négociation du Programme de travail et budget de l'OCDE pour 2013-14, qui a été adopté par le Conseil en décembre. Elle a notamment présenté le premier Plan d'investissement pluriannuel (PIP) relatif au remplacement des immobilisations de l'Organisation. La publication du deuxième rapport biennal sur l'efficience de l'OCDE a révélé une optimisation accrue des ressources au profit de ses membres. L'évolution du rôle de l'Organisation s'est faite sentir au niveau de ses services centraux, avec, notamment, des manifestations de haut niveau organisées en plus grand nombre et à plus grande échelle au siège, et une attention croissante accordée à la sécurité des missions, à l'heure où les agents de l'OCDE collaborent avec un ensemble de plus en plus large de pays souhaitant bénéficier des analyses et des conseils de l'Organisation.

En 2013, la Direction exécutive continuera de s'employer à mieux organiser et exploiter la masse de connaissances que recèle l'Organisation, et à encourager les talents. La recherche de gains d'efficience sera encore intensifiée, tant au niveau des opérations courantes que pour la gestion des engagements à long terme. Nous continuerons aussi d'adapter les locaux et la gestion opérationnelle de l'Organisation aux impératifs écologiques, notamment en compensant l'empreinte carbone des missions de l'OCDE. Nous renforcerons enfin la coordination entre les différents services centraux, afin de mieux gérer les priorités et de livrer un message cohérent et homogène sur les politiques et pratiques internes. 


\section{Audit interne et Évaluation}

« L’Audit interne et l'Évaluation

jouent un rôle essentiel dans

la gouvernance de l'OCDE.

L'Audit interne offre au

Secrétaire général une garantie

indépendante quant à la

qualité des contrôles visant à

prévenir un large éventail de

risques. Ses recommandations

contribuent à renforcer la

responsabilité et l'efficience

des directions ainsi que des

principaux services centraux.

L'Évaluation se concentre sur

les réalisations concrètes de

l'Organisation et examine

la pertinence, l'efficacité et

l'efficience des comités de

substance de l'OCDE. Ses

recommandations participent

à l'amélioration des performances

des comités et au renforcement

de leur responsabilité à l'égard

du Conseil. »
Outre les examens annuels de la procédure d'attestation par les managers et des contributions volontaires, les principaux audits internes axés sur les risques ont porté en 2012 sur les projets horizontaux, la sécurité informatique, la procédure de recrutement du personnel, les activités de traduction, les contrôles d'accès au siège et la sécurité des missions du personnel à l'étranger. L'Audit interne a également accompli toute une série de missions consultatives destinées à améliorer le système de gestion des risques et à renforcer l'organisation et les méthodes de travail des directions.

En 2012, les activités d'évaluation ont porté avant tout sur le deuxième cycle d'évaluation en profondeur. S'appuyant sur le premier cycle, consacré pour l'essentiel à l'amélioration du fonctionnement des comités, le deuxième cycle d'évaluation en profondeur s'attache davantage à renforcer leur pertinence et leur efficacité, tout en accélérant et en approfondissant l'incorporation de l'évaluation en profondeur au cycle de gestion intégré de l'Organisation. L'année 2012 a vu par ailleurs la mise en place d'un cadre permettant de recenser, d'analyser et de diffuser les bonnes pratiques des comités et le lancement d'une évaluation pilote de la Stratégie pour l'innovation. 


\section{Direction des affaires juridiques}

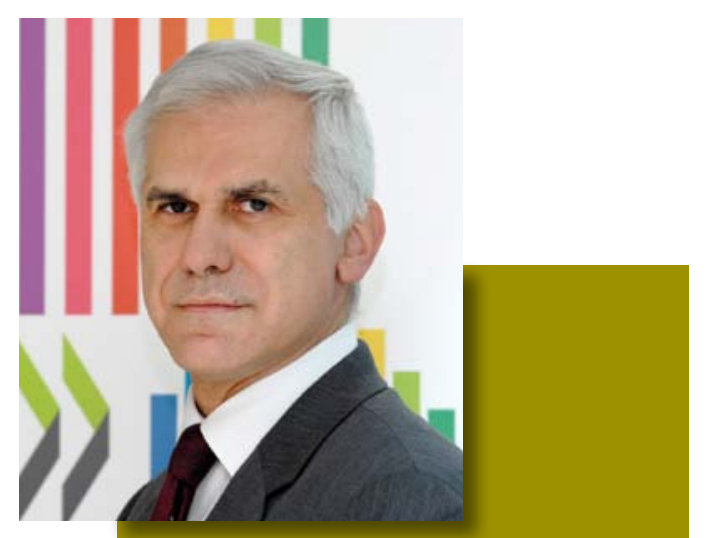

Nicola Bonucci

Directeur

Courriel : legal@oecd.org

« Le droit peut être "mou", mais

le suivi et la mise en œuvre des

recommandations ne le sont pas.

Au cours du processus d'examen

par les pairs, le contrôle et le

suivi garantissent un degré de

compréhension, d'acceptation et

de mise en œuvre probablement

beaucoup plus élevé que

dans les autres organisations

internationales. 》)
La Direction des affaires juridiques a continué avec détermination de coordonner le processus d'adhésion de la Fédération de Russie, en collaboration avec toutes les parties prenantes. La Fédération de Russie est désormais membre de l'Agence de l'OCDE pour l'énergie nucléaire.

Depuis les années 1990, la Direction des affaires juridiques s'est chargée de la préparation des termes, conditions et procédures d'adhésion ainsi que de la coordination technique du processus d'adhésion, grâce à sa vision d'ensemble de l'Organisation et à son expertise des instruments juridiques qui sont au centre des discussions d'adhésion. La Direction agit comme point central de contact et de facilitation pour les pays candidats, ainsi que pour les comités et directions de l'OCDE qui entreprennent les examens d'adhésion, et fait part au Conseil des progrès réalisés. L’année passée, la direction des affaires juridiques a activement contribué aux discussions concernant l'adhésion de la Fédération de Russie, qui se sont intensifiées, avec 22 examens techniques parallèles de comités et des contacts à haut niveau avec les autorités russes. La Direction a aussi activement contribué aux débats concernant les nouvelles demandes d'ouverture de discussions d'adhésion.

La Direction a apporté son soutien aux travaux de l'Organisation dans le domaine fiscal, en lui prêtant son concours pour le projet relatif à l'érosion de la base d'imposition et au transfert de bénéfices, pour l'organisation du Forum mondial sur la transparence et l'échange de renseignements en matière fiscale, ainsi que pour la révision de la Convention concernant l'assistance administrative mutuelle en matière fiscale. Cette Convention compte désormais 45 signataires, qui seront bientôt rejoints par d'autres pays.

La Direction a également contribué à lélaboration de divers instruments juridiques portant sur les essais cliniques, les bioproduits, la protection financière des consommateurs, les marchés publics, la pêche, la politique de la réglementation, les crédits à l'exportation et la gouvernance des Codes de la libération des mouvements de capitaux et des opérations invisibles courantes.

Elle participe aux discussions relatives à l'élargissement possible de l'Organisation et à sa gouvernance engagée dans ce contexte. Elle formule des avis sur l'engagement envers les non membres et a participé aux travaux sur la nouvelle Résolution sur les partenariats, déterminant leur participation dans les organismes de l'OCDE. Enfin, elle coordonne les efforts pour protéger les noms/acronymes des OIG dans le cadre de la nouvelle initiative sur les domaines de premier niveau génériques (gTLDs) lancée par l'ICANN (Société pour l'attribution des noms de domaine et des numéros sur Internet).

La Direction formule des avis sur les aspects juridiques, institutionnels et procéduraux des activités de l'Organisation, notamment dans le cadre de l'élaboration de ses instruments juridiques. Elle apporte un soutien juridique en matière de contrats, d'accords de coopération, de contributions volontaires et de propriété intellectuelle. Enfin, elle représente l'Organisation en justice et soutient les réformes menées dans le domaine financier et celui des ressources humaines. 


\section{Secrétariat du Conseil et du Comité exécutif}

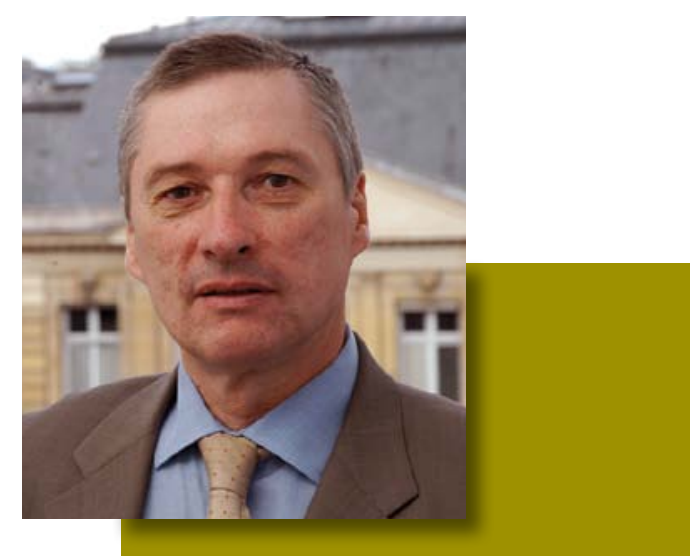

Patrick van Haute Directeur

« Le Secrétariat du Conseil continuera à assurer l'interface entre les membres et le Secrétariat, à préparer les décisions qui dessineront l'avenir de l'OCDE. En cette période de difficultés économiques pour les membres, la valeur ajoutée potentielle de l'OCDE est plus forte que jamais. Notre travail et nos compétences sont dédiés à la mise en œuvre de nos recommandations, pour le bénéfice de nos concitoyens. $\gg$
Le Secrétariat du Conseil est au service du Conseil et de ses Comités permanents, des réunions des Chefs de Délégations, du Groupe de Stratégie globale et de la Réunion du Conseil au niveau des ministres (RCM). Le Secrétariat du Conseil conseille le Secrétaire général sur la politique générale de l'Organisation, fournit des avis et une assistance sur les aspects des activités du Conseil concernant les institutions, les politiques et les procédures. Il assure également la liaison entre le Secrétariat et les Délégations des pays membres pour la gestion du programme de travail du Conseil. Le Secrétariat du Conseil est au service du Conseil et du Comité exécutif pour leurs activités quotidiennes. Le Conseil, ainsi que le Comité exécutif, sont particulièrement efficaces, parvenant au consensus nécessaire pour prendre les décisions en temps opportun et de manière optimale. Le dialogue du Secrétariat du Conseil avec les Directions facilite leur engagement auprès des membres.

Dans la limite des ressources disponibles, le Secrétariat du Conseil continuera d'améliorer ses services auprès du Conseil, de sa Présidence et du Comité exécutif, en progressant dans la chaîne de valeur grâce au recueil d'informations opportunes auprès des différents réseaux, à la diffusion de ces informations et à leur intégration dans le processus de production de documents. 



\section{AGENCES \\ DE L'OCDE ET \\ AUTRES ENTITÉS SPÉCIALES}

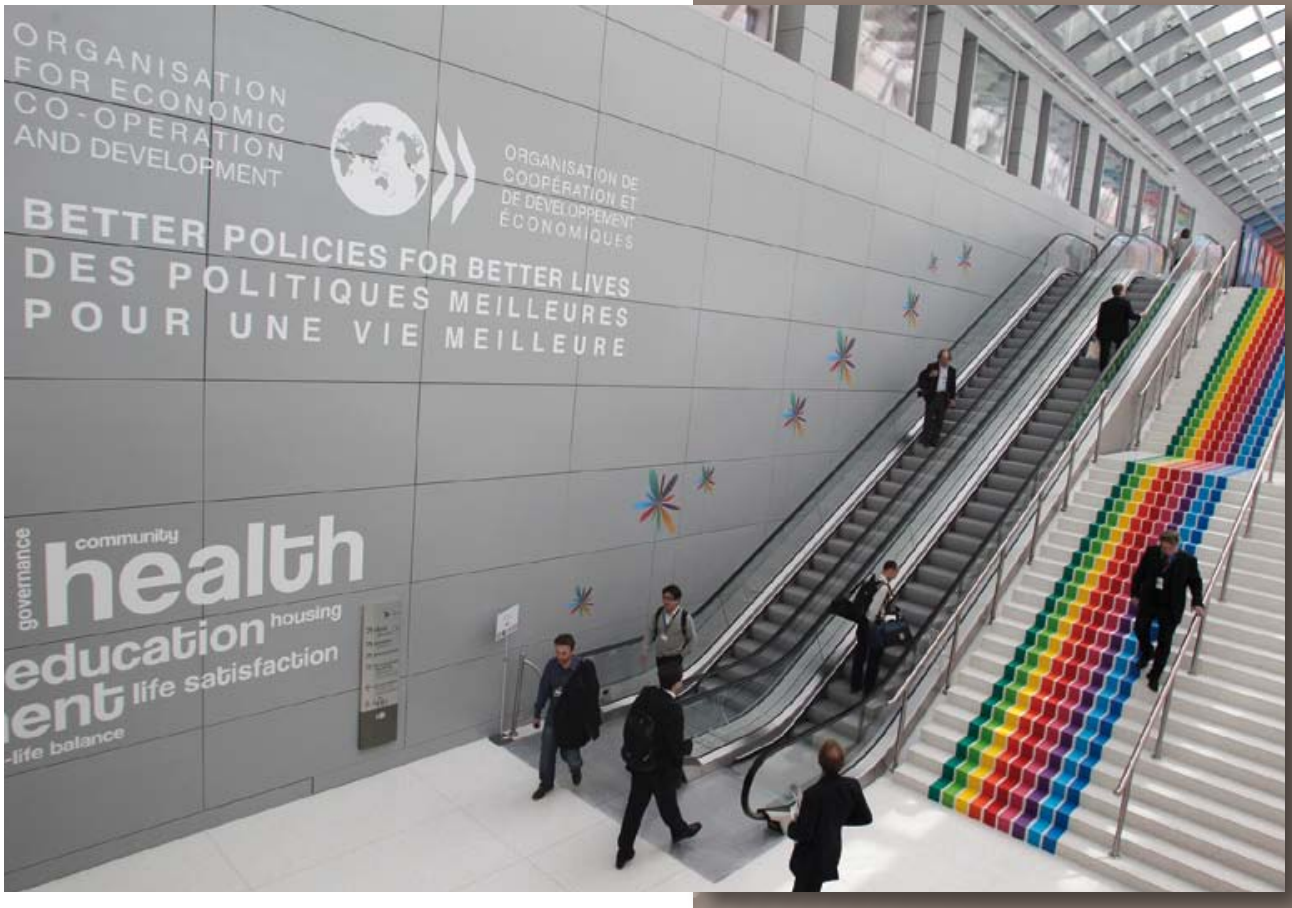




\section{Centre de développement}

Le Centre de développement de l'OCDE, créé en 1962, poursuit ses activités autour de deux axes qui se renforcent mutuellement : une recherche pluridisciplinaire et un dialogue sur les politiques fondé sur des données factuelles. L'axe de recherche porte essentiellement sur la cohésion sociale, la compétitivité et la croissance. Le dialogue sur les politiques offre un espace d'échange aux 41 membres, dont 24 pays de l'OCDE et 17 économies émergentes et en développement.

À travers cette recherche pluridisciplinaire et ce dialogue étayé par des données factuelles, le Centre identifie à l'intention des pouvoirs publics des solutions efficaces concernant la croissance, la lutte contre la pauvreté et la réduction des inégalités, selon les différents contextes des pays membres. Des initiatives comme les forums régionaux annuels consacrés à l'Afrique et à l'Amérique latine ou le Forum mondial de l'OCDE sur le développement permettent au Centre d'agir dans le domaine du développement aux côtés de ses partenaires stratégiques. Parallèlement, les partenariats noués avec des acteurs régionaux, comme les commissions économiques des Nations unies et les banques régionales de développement, alimentent les analyses macroéconomiques et stratégiques régionales servant de base aux publications annuelles du Centre : les Perspectives économiques en Afrique, les Perspectives économiques de l'Amérique latine, les Perspectives économiques en Asie du Sud-Est et les Perspectives du développement mondial.

En tant qu'important contributeur à la Stratégie de l'OCDE pour le développement, le Centre propose des outils pour aider les pays à concevoir des réformes et des stratégies de mise en œuvre efficaces, notamment: les Examens multidimensionnels par pays, qui visent à identifier les obstacles au développement et à proposer des stratégies nationales; des plateformes de dialogue entre des pays confrontés à des difficultés structurelles semblables; et des plateformes de dialogue au service du suivi économique régional, qui concourent à l'intégration régionale et à un développement économique équitable, notamment en Asie du Sud-Est.

Pour plus d'informations, voir : www.oecd.org/dev-fr

\section{Agence pour l'énergie nucléaire}

L'Agence pour l'énergie nucléaire (AEN) est une agence spécialisée de l'OCDE. Ses 31 pays membres, depuis l'adhésion de la Fédération de Russie le $1^{\text {er }}$ janvier 2013, représentent environ $90 \%$ de la puissance nucléaire installée mondiale. L'AEN aide ses pays membres à maintenir et à approfondir, par l'intermédiaire de la coopération internationale, les bases scientifiques, technologiques et juridiques nécessaires à une utilisation sûre, respectueuse de l'environnement et économique de l'énergie nucléaire à des fins pacifiques. Ses travaux nourrissent les décisions publiques sur les politiques nucléaires, ainsi que les analyses stratégiques plus générales de l'OCDE. Ses domaines d'activité sont la sûreté et la réglementation nucléaires, la gestion des déchets radioactifs, la protection radiologique, les sciences et données nucléaires, le développement de l'énergie nucléaire et le cycle du combustible, ainsi que les affaires juridiques. Elle mène, par ailleurs, une série de projets de recherche internationaux et assure le secrétariat technique de deux initiatives majeures, le Programme multinational d'évaluation des conceptions, qui vise à renforcer la coopération pour l'analyse de sûreté des futurs réacteurs, et le Forum international Génération IV, qui coordonne les efforts de recherche et de développement consacrés par les membres de l'AEN aux systèmes nucléaires de la prochaine génération.

Depuis l'accident nucléaire de Fukushima en mars 2011, l'AEN prête directement assistance aux autorités japonaises pour la conception et la réalisation des évaluations de sûreté et des tests de résistance, la définition des principaux éléments d'une réforme efficace de la réglementation du pays, l'utilisation des bonnes pratiques de remise en état des sols contaminés par des substances radioactives, la planification et la gestion efficace des activités de décontamination, mais aussi la planification à long terme du démantèlement de la centrale. L'AEN a organisé de nombreuses activités internationales, dont des projets de recherche communs et des réunions avec des autorités réglementaires, des spécialistes en sûreté nucléaire et santé publique et des représentants de la société civile. Une synthèse des principales activités de l'AEN et des pays membres, entreprise à la suite de l'accident de Fukushima Daiichi, doit paraître au milieu de l'année. Une section du site Internet de l'AEN a été réservée aux échanges d'information entre pays membres.

Pour plus d'informations, voir : www.oecd-nea.org/nea/index-fr.html 


\section{Agence internationale de l'énergie}

La carte mondiale de l'énergie est en train de changer. C'était l'une des conclusions claires de l'analyse de l'AIE en 2012, et un thème repris par l'Agence dans ses différents rapports, interventions publiques et consultations politiques. Les changements en cours transformeront les rôles des différents pays, régions et formes d'énergie dans les prochaines décennies.

Les travaux de l'AIE ont porté sur la sécurité énergétique, la viabilité écologique, le développement économique et l'engagement dans le monde entier. Les activités d'analyse des marchés, de préparation aux procédures d'urgence, d'ouverture internationale et de gestion des statistiques de l'énergie ont appuyé ces travaux.

L'édition 2012 du World Energy Outlook a battu des records de ventes et reçu un accueil élogieux dans les pays membres et partenaires. Elle a mis l'accent sur l'efficacité énergétique, l'extraction de pétrole et de gaz non conventionnels (et notamment sur les règles d'or pour soutenir ces "révolutions ") et l'Irak, qui pourrait changer la donne sur les marchés pétroliers mondiaux.

La série de l'AIE Medium-Term Market Report comporte désormais des prévisions annuelles à cinq ans sur le pétrole, le gaz, le charbon et les énergies renouvelables. L'efficacité énergétique - « l'énergie cachée » - y sera ajoutée en 2013.

L'édition 2012 de la série bisannuelle Energy Technology Perspectives a continué de traiter le thème du développement des technologies énergétiques propres, et présenté des perspectives à l'horizon 2050. Il en ressort que les technologies éolienne et solaire sont sur la bonne voie pour contribuer à la réalisation des objectifs climatiques à long terme, mais que d'autres secteurs sont à la traîne, en raison surtout de faiblesses de l'action publique.

Les partenariats extérieurs ont été consolidés (notamment avec des partenaires clés comme la République populaire de Chine, l'Inde, la Fédération de Russie, le Brésil, le Mexique, l'Indonésie et l'Afrique du Sud), à la suite de l'adoption d'une stratégie de coopération mondiale renforcée (Global Engagement Strategy) et des principes directeurs pour sa mise en œuvre. Des non membres ont été associés à des exercices de préparation aux procédures d'urgence en cas de perturbations des approvisionnements pétroliers et gaziers, et des exercices et manifestations sur mesure ont été organisés à Delhi, Beijing et Bangkok.

L'ensemble de nos travaux bénéficie de l'amélioration permanente de la collecte et de la gestion de données (et notamment du soutien à la Joint Organisations Data Initiative) et s'appuie sur une stratégie de communication visant à maximiser l'impact des activités de l'AIE. L'une et l'autre ont beaucoup compté dans l'augmentation des recettes de publications, qui ont atteint leur niveau le plus élevé.

Pour plus d'informations, voir : www.iea.org 


\section{Forum pour le partenariat avec l'Afrique}

Le Forum pour la partenariat avec l'Afrique (FPA) est une tribune politique de premier plan qui débat des grandes priorités de l'action publique et suit les progrès des engagements pour le développement de l'Afrique. Il a été créé en 2003 après le Sommet du G8 à Évian, afin d'élargir le dialogue entre le G8 et le Nouveau partenariat pour le développement de l'Afrique (NEPAD) en y associant les principaux partenaires bilatéraux et multilatéraux au développement de l'Afrique. Il regroupe l'ensemble des pays africains membres du NEPAD, les principales institutions africaines et d'autres partenaires majeurs au développement. En 2005, les membres du FPA ont decidé de créer une unité de soutien, hébergée par l'OCDE, remplissant le rôle de Secrétariat et initiant les études de suivi et d'analyse.

Le FPA s'est réuni à deux reprises en 2012 : en avril (à Paris) pour débattre de questions relatives à l'énergie, et en novembre (à Cotonou, Bénin) pour discuter des flux financiers illicites. Il s'est également réuni à Londres en avril 2013 pour débattre des quesitons de transparence avant le Sommet du G8 à Lough Erne. Avec la Commission économique des Nations unies pour l'Afrique, l'unité de soutien a préparé les éditions 2012 et 2013 de l'Examen mutuel de l'efficacité du développement en Afrique, qui analyse la mise en œuvre des engagements pris par les pays africains et leurs partenaires, les résultats obtenus et les priorités futures. Le rapport 2013 a été publié pour la réunion de Londres. L’unité de soutien est également impliquée dans le travail général de l'OCDE sur la stratégie de développement.

Pour plus d'information, voir : www.africapartnershipforum.org/fr

\section{Groupe d'action financière}

Le Groupe d'action financière (GAFI) est un organisme intergouvernemental indépendant qui définit des normes internationales de lutte contre le blanchiment de capitaux et le financement du terrorisme, ainsi que contre la prolifération d'armes de destruction massive.

En février 2013, le GAFI a adopté la Méthodologie d'évaluation de la conformité technique avec les Recommandations du GAFI. Cette méthodologie propose un cadre pour déterminer dans quelle mesure les pays atteignent l'objectif de lutte contre le blanchiment de capitaux et le financement du terrorisme. Les évaluations futures se concentreront sur le respect technique des obligations spécifiques attachées à chacune des Recommandations du GAFI et sur l'efficacité des systèmes nationaux de lutte contre le blanchiment de capitaux et le financement du terrorisme (LBC/FT).

Le GAFI a par ailleurs publié National Money Laundering and Terrorist Financing Risk Assessments, orientations qui aident les pays à mieux comprendre les risques auxquels ils sont confrontés, afin de pouvoir ensuite adapter leurs systèmes de LBC/ FT en conséquence. Le GAFI a également publié en 2011 une version actualisée de ses Lignes directrices sur la lutte contre le blanchiment de capitaux et le financement du terrorisme et la finance inclusive, qui mettent l'accent sur les moyens de concilier intégrité financière et accès aux services financiers. Ces deux rapports ont été publiés en février 2013.

En juin 2012, M. Bjørn Skogstad Aamo est devenu Président du GAFI. Depuis mai 2012, le Groupe d'action financière compte par ailleurs deux nouveaux observateurs : le Comité de liaison anti-blanchiment de la Zone franc (CLAB), qui contribuera à promouvoir la mise en place de dispositifs de LBC/FT dans les quinze pays africains qui en sont membres, et l'Organisation pour la sécurité et la coopération en Europe (OSCE), qui permettra au GAFI d'élargir la portée de son action internationale à tout le continent européen.

Pour plus d'informations, voir : www.fatf-gafi.org 


\section{Forum international des transports}

Le Forum international des transports (FIT) est une organisation intergouvernementale rattachée à l'OCDE qui regroupe 54 pays membres. Sa mission est de faire progresser les politiques et les pratiques dans le secteur des transports afin que ceux-ci favorisent la croissance économique, la protection de l'environnement et la cohésion sociale. Dans cet esprit, le FIT joue le rôle de laboratoire d'idées pour les responsables de l'élaboration des politiques et la communauté internationale des transports, en permettant de mieux cerner, à l'aide de données factuelles, les questions relevant de la politique des transports. Ses travaux s'appuient sur des recherches économiques, la collecte de statistiques et l'analyse des politiques.

Les groupes de travail rassemblent les connaissances acquises au niveau mondial sur des sujets allant de " L'adaptation des infrastructures de transport au changement climatique " à « La sécurité des piétons, l'espace urbain et la santé ». Les tables rondes récentes portaient par exemple sur les thèmes suivants : " Accroître la capacité aéroportuaire sous les contraintes des grandes zones urbaines ", "Les tendances à long terme de la demande de mobilité » et «La gestion des risques et le biais d'optimisme dans les PPP ».

Le FIT organise également un Sommet annuel pour les ministres des Transports. Ce Sommet s'est imposé comme principale plateforme mondiale de débat à haut niveau sur la politique des transports et réunit les ministres des pays membres du FIT ainsi que des décideurs du secteur, de la société civile et du monde de la recherche.

En mai 2012, le Sommet annuel sur « Des transports sans rupture : développer les connections » a rassemblé un nombre sans précédent de 1000 délégués à Leipzig, en Allemagne. Les ministres ont appelé à poursuivre « l'ambition stratégique forte et ambitieuse » d'une connectivité sans rupture, favorisant une meilleure mobilité et une croissance économique durable. Le Secrétaire général de l'OCDE Angel Gurría y a prononcé un discours liminaire et présenté l'édition 2012 des Perspectives des transports du FIT, intitulée Des transports sans rupture au service d'une croissance verte. Dans le cadre de ce Sommet, José Viegas, du Portugal, a été élu Secrétaire général du FIT.

Le Sommet 2013 du Forum international des transports s'est tenu du 22 au 24 mai autour du thème "Financer les transports ".

Pour plus d'informations, voir : www.internationaltransportforum.org

\section{Club du Sahel et de l'Afrique de l'Ouest}

Le Club du Sahel et de l'Afrique de l'Ouest (CSAO) est la plus ancienne initiative de solidarité et de partenariat entre l'OCDE et l'Afrique. Il a été créé en 1976 par des pays membres de l'OCDE pour mobiliser le soutien et l'attention de la communauté internationale sur les sécheresses frappant la région du Sahel. Face à l'interdépendance croissante des pays de la région, son champ géographique a été étendu à l'ensemble des pays de l'Afrique de l'Ouest en 2001. Fondé sur le principe du partage des connaissances et des expériences, le Club met ses ressources au service de programmes et du leadership des organisations régionales de l'Afrique de l'Ouest. En 2011, la Communauté des États d'Afrique de l'Ouest (CEDEAO), l'Union économique et monétaire ouest-africaine (UEMOA) et le Comité permanent inter-États de lutte contre la sécheresse au Sahel (CILSS) sont devenus membres du Club. Les membres du CSAO sont déterminés à travailler ensemble au développement et à l'intégration de l'Afrique occidentale. Dans cette optique, le Club suit de près les évolutions socioéconomiques, politiques et environnementales de la région, mène des analyses prospectives et promeut le dialogue. En 2013 et 2014, le Secrétariat du CSAO continuera à se concentrer sur la gouvernance régionale de la sécurité alimentaire et nutritionnelle. Plus particulièrement, il prête son concours à l'Alliance globale pour l'Initiative Résilience (AGIR) lancée à Ouagadougou en décembre 2012. Une nouvelle plateforme de réflexion sur les zones saharo-sahéliennes, et plus particulièrement sur le pastoralisme transhumant, sera ouverte. Ces deux axes de travail seront étayés et complétés par des activités transversales visant principalement à accroître l'impact des travaux du CSAO sur la formulation des politiques et sur l'assistance aux membres. 


\section{Comité consultatif économique et industriel}

Fondé en 1962, le Comité consultatif économique et industriel auprès de l'OCDE (BIAC) est officiellement reconnu par le Conseil de l'OCDE comme l'organe représentatif des milieux d'affaires des pays de l'OCDE.

Le BIAC apporte un point de vue indépendant, intersectoriel et pluridisciplinaire sur les travaux de l'OCDE. Il rassemble plus de 2800 représentants des milieux d'affaires, issus de 52 organisations patronales nationales des pays membres de l'OCDE et des principales économies non membres, et plus de 35 associations sectorielles supranationales. Les membres du BIAC collaborent dans le cadre de 38 groupes d'orientation différents couvrant tout l'éventail des politiques inspirées par l'OCDE. En tant qu'association patronale internationale indépendante, le BIAC préconise, au nom des industriels, des positions consensuelles auprès de l'OCDE et de ses États membres, pour que les instruments d'action adoptés favorisent une croissance et une prospérité tirées par le secteur privé.

Durant l'année écoulée, la première priorité du BIAC a été d'œuvrer avec l'OCDE à promouvoir les créations d'emplois et une croissance économique mondiale vigoureuse, tirée par l'investissement privé. Il a exhorté les gouvernements à mener des politiques en faveur de l'employabilité, de l'innovation, de la croissance verte et de l'ouverture des échanges et de l'investissement. Le BIAC n'a cessé de souligner le fait que le meilleur moyen de fortifier l'activité économique est de privilégier les valeurs fondamentales de l'économie de marché.

Face à l'environnement mondialisé des entreprises, le BIAC entend continuer à renforcer la coopération et la coordination avec les milieux d'affaires des pays non membres, et encourager ainsi ces derniers à s'engager auprès de l'OCDE.

Pour plus d'informations, voir : www.biac.org

\section{Commission syndicale consultative}

La Commission syndicale consultative auprès de l'OCDE (TUAC) est l'organe de liaison avec le mouvement syndical. Le TUAC comprend 59 centrales syndicales affiliées dans les pays de l'OCDE, représentant plus de 61 millions de travailleurs, ainsi que des membres associés en Afrique du Sud, au Brésil, en Indonésie et en Russie. Durant l'année écoulée, le TUAC s'est concentré sur la réponse à la crise économique, en soulignant la nécessité d'adopter des mesures propres à stimuler la croissance, à protéger et développer l'emploi, et à renforcer la réglementation du secteur financier. Il préconise un changement radical d'orientation du modèle économique, afin d'établir une économie mondialisée plus forte, permettant de réduire les inégalités de revenus.

Le TUAC, en collaboration avec la Confédération syndicale internationale, a coordonné les contributions des syndicats aux sommets du G20 et du G8, par l'intermédiaire du L20, et a pris part aux travaux du groupe de travail du G20 sur l'emploi, ainsi qu'aux conférences sur ce thème. Il a également coordonné les contributions des syndicats à l'ouvrage Exiting from the Crisis: Towards a Model of More Equitable and Sustainable Growth.

Le TUAC et ses partenaires de Global Union ont aussi contribué à la mise à jour des Principes directeurs de l'OCDE à l'intention des entreprises multinationales, et le TUAC a créé une base de données et un site Internet regroupant les cas relevant des Principes directeurs depuis 2000.

Les membres du Bureau du TUAC sont : Président : Richard Trumka, Président de l'AFL-CIO, États-Unis ; Vice-présidents : Marc Leemans, Président de la CSC, Belgique ; Marie-Louise Knuppert, Secrétaire nationale de LO-Danemark ; Nobuaki Koga, Président de RENGO, Japon ; Secrétaire général : John Evans.

Pour plus d'informations, voir : www.tuac.org 


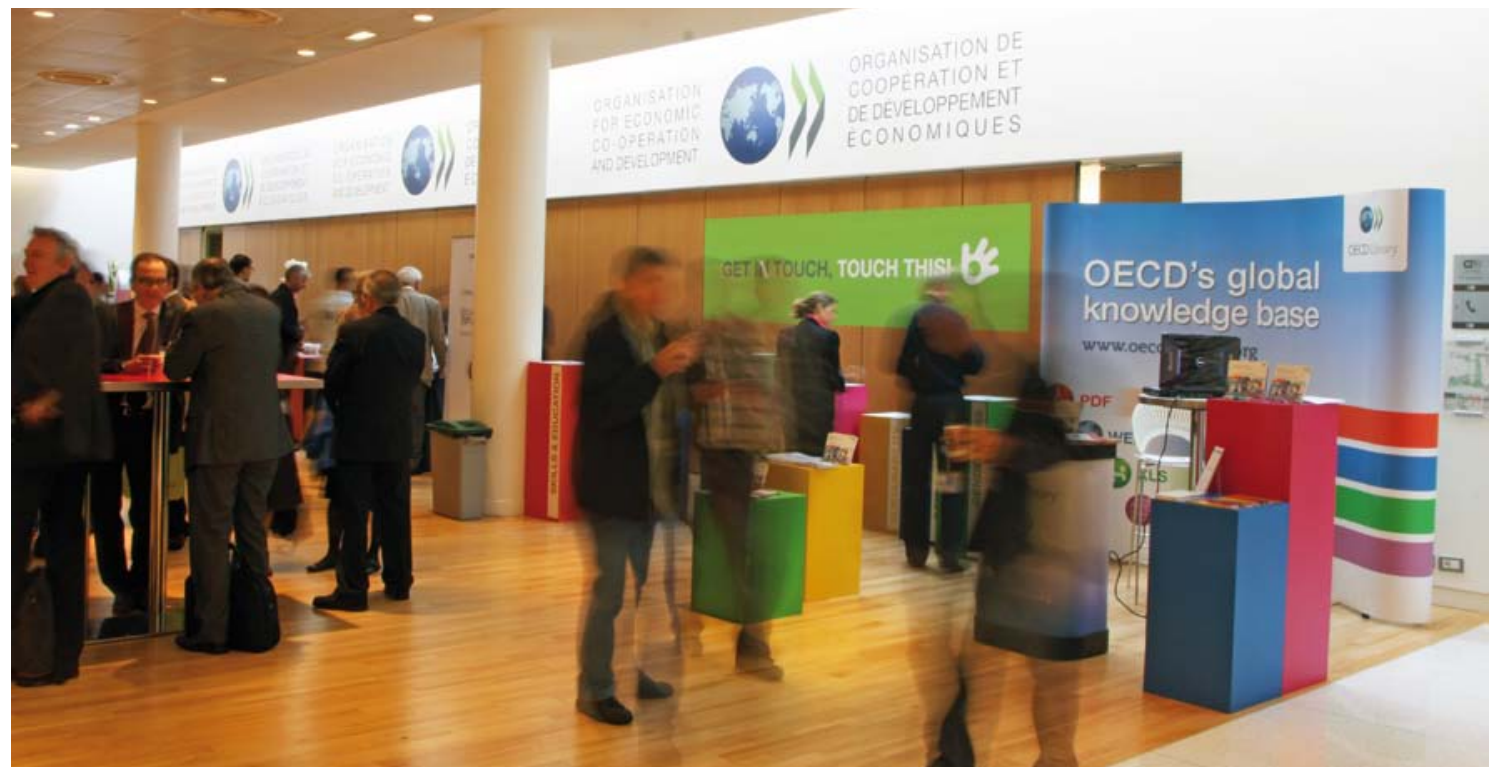

\section{Gestion financière et budgétaire}

\section{www.oecd.org/budget-fr}

financeservice@oecd.org

L'Organisation applique le principe d'une gestion axée sur les résultats à ses activités de planification, d'établissement du budget et de gestion financière, en déterminant quels sont les effets sur l'action des pouvoirs publics recherchés par ses membres, en déployant les ressources avec flexibilité pour obtenir ces résultats eu égard au caractère évolutif du programme de travail, et en évaluant l'exécution du budget a posteriori. Le budget de l'OCDE et la teneur de son programme de travail sont établis tous les deux ans par l'organe directeur de l'Organisation (le Conseil), à partir des recommandations du Secrétaire général. Le budget 2013 s'élève à 354 millions EUR, augmenté d'environ un tiers par des contributions volontaires, ressources extrabudgétaires planifiées mais variables, fournies principalement par des organismes gouvernementaux et des institutions.

Les états financiers de l'OCDE sont préparés conformément aux Normes comptables internationales pour le secteur public (IPSAS) et vérifiés par un auditeur externe choisi parmi les organismes de contrôle des pays membres de l'OCDE. Les états financiers de l'Organisation sont disponibles sur le site Internet de l'OCDE.

\section{Contributions statutaires} des pays membres pour 2013

Tous les pays membres contribuent aux réalisations financées par la Partie I du budget, qui représentent $53 \%$ du budget total de l'Organisation. Les réalisations de la Partie II , qui intéressent un nombre plus restreint de pays membres ou touchent à des domaines particuliers d'action des pouvoirs publics non couverts par la Partie I, représentent $26 \%$ du budget total. Le reste recouvre d'autres budgets, tels que le budget des pensions et les budgets spéciaux. Le calcul des contributions à la Partie I repose à la fois sur la répartition d'un montant à parts égales entre tous les pays membres et sur l'application d'un barème tenant compte de la taille relative des économies membres (suivant le critère du revenu national). Les programmes de la Partie II sont financés selon un barème ou un autre dispositif convenu entre les pays participants. 



\section{ORGANISATION DE COOPÉRATION ET DE DÉVELOPPEMENT ÉCONOMIQUES}

L'OCDE est un forum unique en son genre où les gouvernements œuvrent ensemble pour relever les défis économiques, sociaux et environnementaux que pose la mondialisation. L'OCDE est aussi à l'avant-garde des efforts entrepris pour comprendre les évolutions du monde actuel et les préoccupations qu'elles font naître. Elle aide les gouvernements à faire face à des situations nouvelles en examinant des thèmes tels que le gouvernement d'entreprise, l'économie de l'information et les défis posés par le vieillissement de la population. L'Organisation offre aux gouvernements un cadre leur permettant de comparer leurs expériences en matière de politiques, de chercher des réponses à des problèmes communs, d'identifier les bonnes pratiques et de travailler à la coordination des politiques nationales et internationales.

Les pays membres de l'OCDE sont : l'Allemagne, l'Australie, l'Autriche, la Belgique, le Canada, le Chili, la Corée, le Danemark, l'Espagne, l'Estonie, les États-Unis, la Finlande, la France, la Grèce, la Hongrie, l'Irlande, l'Islande, Israël, l'Italie, le Japon, le Luxembourg, le Mexique, la Norvège, la Nouvelle-Zélande, les Pays-Bas, la Pologne, le Portugal, la République slovaque, la République tchèque, le Royaume-Uni, la Slovénie, la Suède, la Suisse et la Turquie. L'Union européenne participe aux travaux de l'OCDE.

Les Éditions OCDE assurent une large diffusion aux travaux de l'Organisation. Ces derniers comprennent les résultats de l'activité de collecte de statistiques, les travaux de recherche menés sur des questions économiques, sociales et environnementales, ainsi que les conventions, les principes directeurs et les modèles développés par les pays membres. 


\section{OCDE Paris}

2, rue André-Pascal, 75775 Paris Cedex 16

Tél : 33 (0) 145248167

Fax : 33 (0) 145249930

E-mail : sales@oecd.org

Online Ordering : www.oecd.org/bookshop

Centre OCDE de Berlin

Schumannstrasse 10, D-10117 Berlin

Tél : 49302888353

Fax : 493028883545

E-mail: berlin.centre@oecd.org

Internet : www.oecd.org/berlin
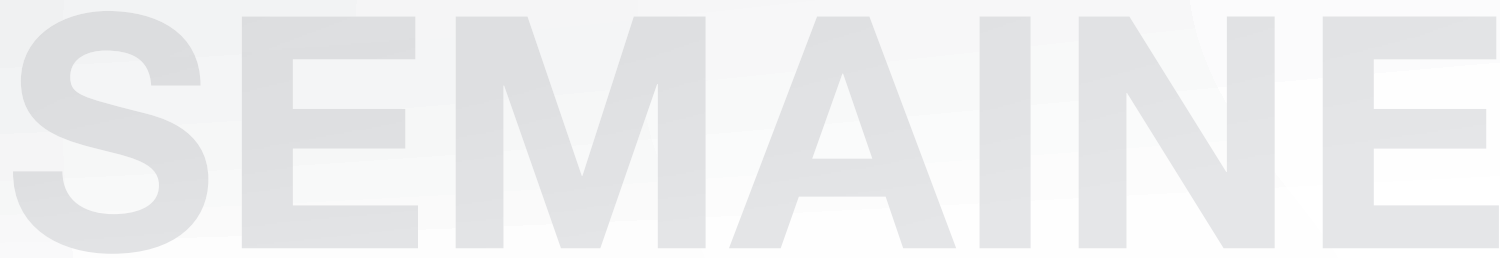

DANS LE MONDE
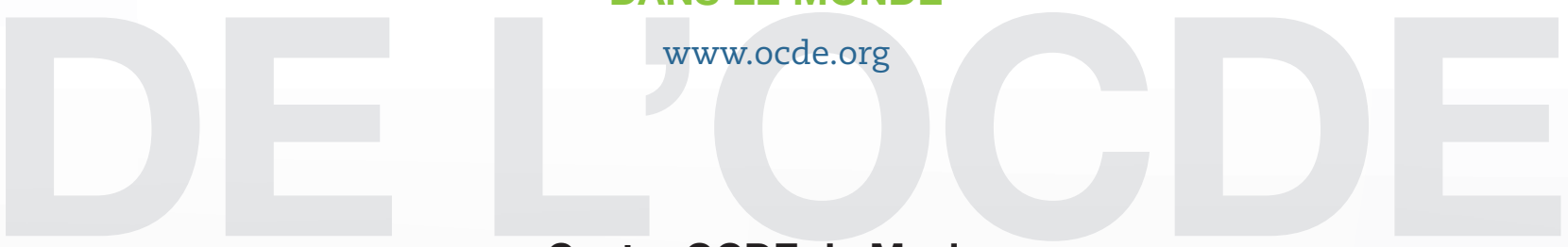

\section{Centre OCDE de Mexico}

Av. Presidente Mazaryk 526

Piso 1, Col. Polanco, México, D.F. 11560

Tél : 525591386230

Fax : 525591387096

E-mail : mexico.contact@oecd.org

Internet : www.oecd.org/centrodemexico

\section{Centre OCDE de Tokyo}

3rd Floor, Nippon Press Center Building 2-2-1 Uchisaiwaicho

Chiyoda-ku, Tokyo 100-0011

Tél : 81355320021

Fax : 81355320035

E-mail : tokyo.contact@oecd.org

Internet : www.oecdtokyo.org

\section{Centre OCDE de Washington}

2001 L Street, NW, Suite 650, Washington DC 20036-4922

Tél : 12027856323

Fax : 12027850350

E-mail : washington.contact@oecd.org

Internet : www.oecdwash.org 